Prepared in cooperation with the United States Environmental Protection Agency's Long Island Sound Study and the Connecticut Department of Energy and Environmental Protection

\title{
Simulation of Groundwater Budgets and Travel Times for Watersheds on the North Shore of Long Island Sound, With Implications for Nitrogen-Transport Studies
}

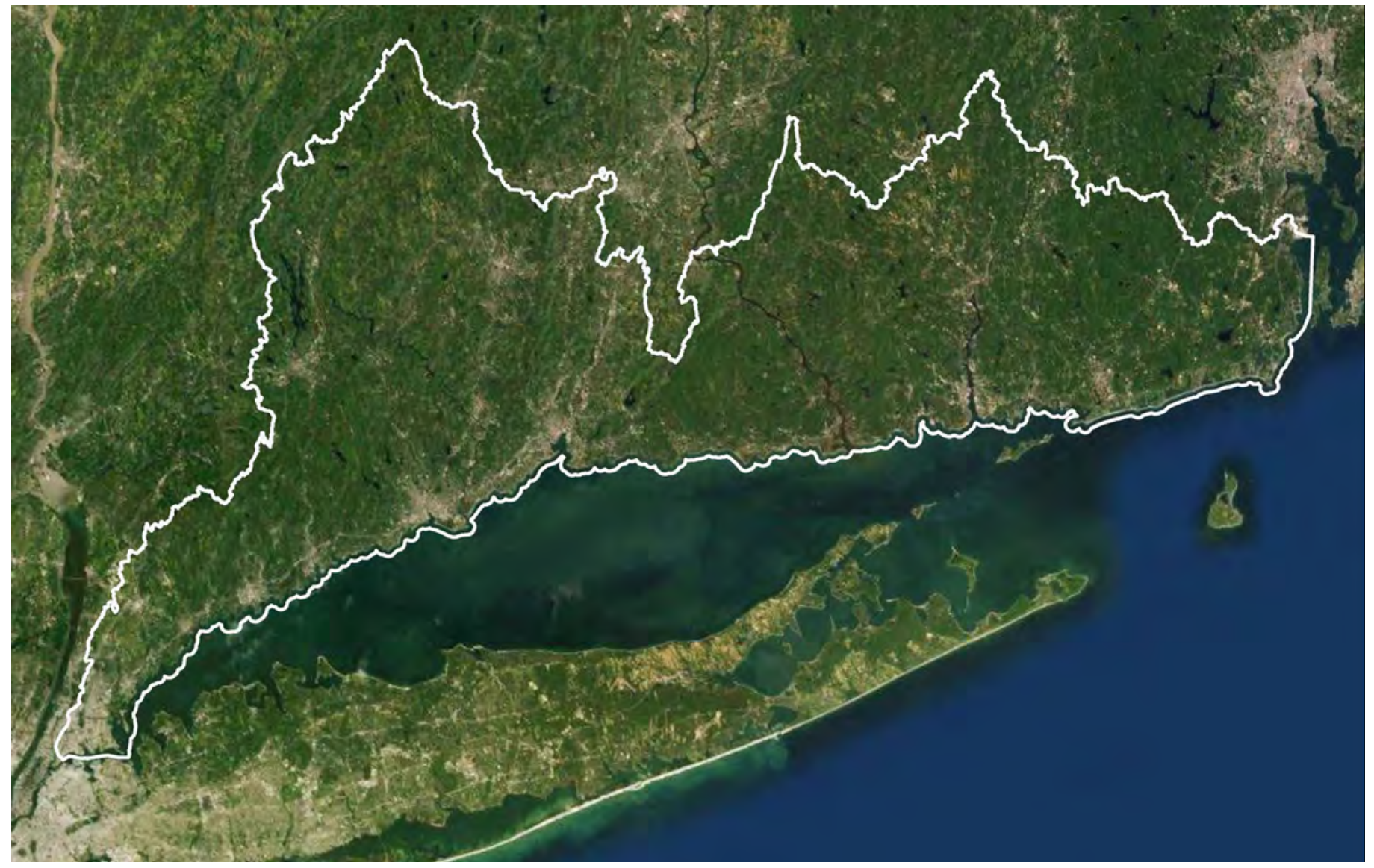

Scientific Investigations Report 2021-5116 
Cover. Aerial view of Long Island Sound and adjacent areas of New York, Connecticut, and Rhode Island, with the study area outlined in white. Map imagery is the intellectual property of Esri and is used herein under license. Copyright 2021 Esri and its licensors. All rights reserved. 


\section{Simulation of Groundwater Budgets and Travel Times for Watersheds on the North Shore of Long Island Sound, With Implications for Nitrogen-Transport Studies}

By Janet R. Barclay and John R. Mullaney

Prepared in cooperation with the United States Environmental Protection Agency's Long Island Sound Study and the Connecticut Department of Energy and Environmental Protection

Scientific Investigations Report 2021-5116 


\section{U.S. Geological Survey, Reston, Virginia: 2021}

For more information on the USGS - the Federal source for science about the Earth, its natural and living resources, natural hazards, and the environment—visit https://www.usgs.gov or call 1-888-ASK-USGS.

For an overview of USGS information products, including maps, imagery, and publications, visit https://store.usgs.gov/.

Any use of trade, firm, or product names is for descriptive purposes only and does not imply endorsement by the U.S. Government.

Although this information product, for the most part, is in the public domain, it also may contain copyrighted materials as noted in the text. Permission to reproduce copyrighted items must be secured from the copyright owner.

Suggested citation:

Barclay, J.R., and Mullaney, J.R., 2021, Simulation of groundwater budgets and travel times for watersheds on the north shore of Long Island Sound, with implications for nitrogen-transport studies: U.S. Geological Survey Scientific Investigations Report 2021-5116, 84 p., https://doi.org/10.3133/sir20215116.

Associated data for this publication:

Barclay, J.R., and Mullaney, J.R., 2021, MODFLOW-NWT and MODPATH groundwater flow models of steady-state conditions in coastal Connecticut and adjacent areas of New York and Rhode Island, as well as a nitrogen transport model of the Niantic River watershed: U.S. Geological Survey data release, https://doi.org/10.5066/P9BLHPIT.

Barclay, J.R., and Mullaney, J.R., 2021, Summary data on groundwater budgets and travel times for watersheds on the north shore of Long Island Sound: U.S. Geological Survey data release, https://doi.org/10.5066/P91T0895.

ISSN 2328-0328 (online) 


\section{Acknowledgments}

The authors would like to thank Kelly Streich and Corinne Fitting from the Connecticut Department of Energy and Environmental Protection, and the Long Island Sound Study for helpful feedback and assistance with this project.

The authors also would like to thank Linda Woolfenden, John Masterson, and Leslie DeSimone for their helpful feedback on an early draft of this report. Finally, the authors would like to recognize the dedicated hydrologic technicians and hydrologists who collected the water-level and streamflow data used to calibrate the model described in this report. 



\section{Contents}

Acknowledgments ……...................................................................................................................

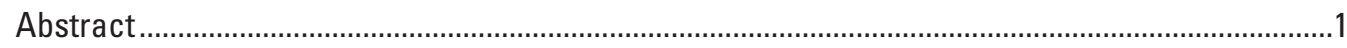

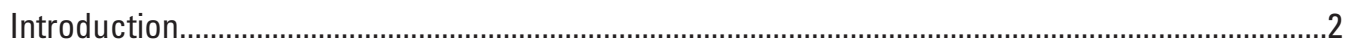

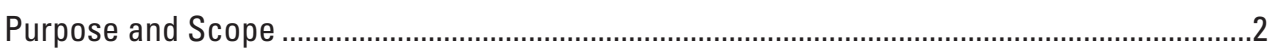

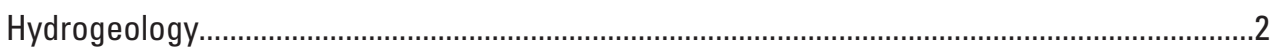

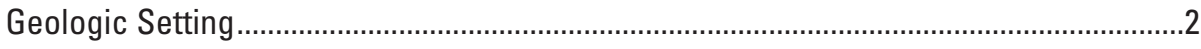

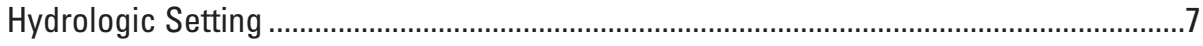

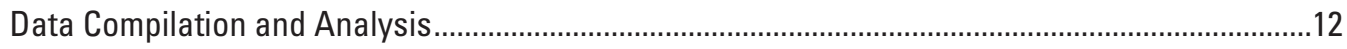

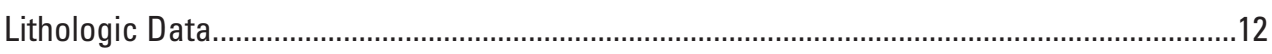

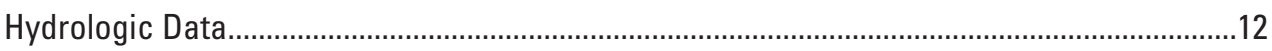

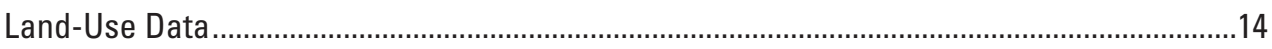

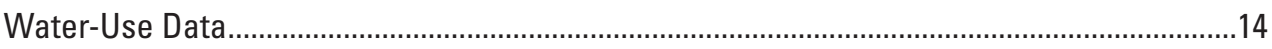

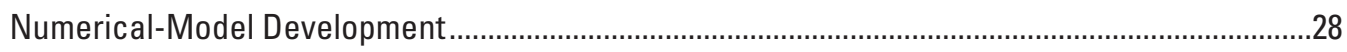

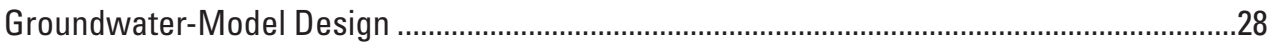

Spatial Extent and Horizontal Model Grid....................................................................28

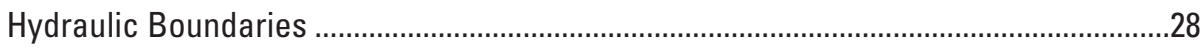

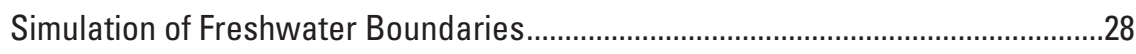

Simulation of the Marine Boundary....................................................................29

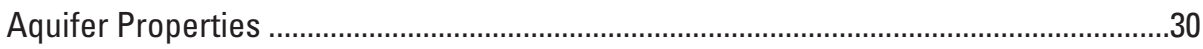

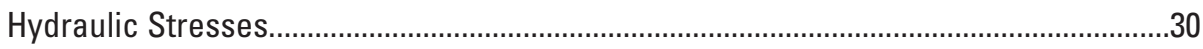

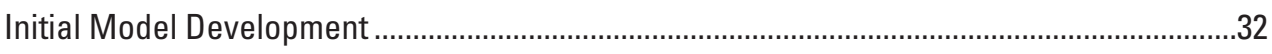

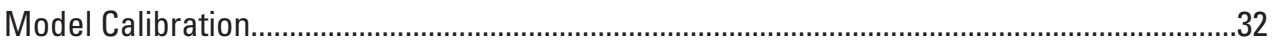

Groundwater-Recharge Calibration ........................................................................

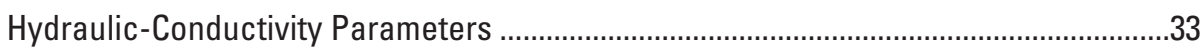

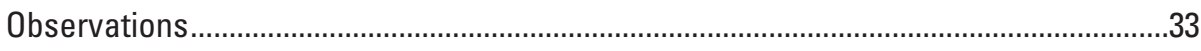

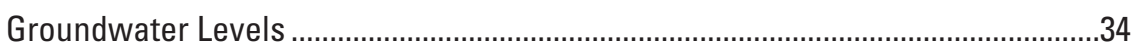

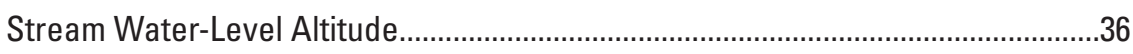

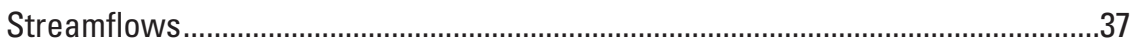

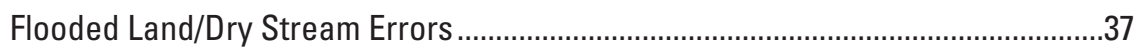

Calibration Results......................................................................................................

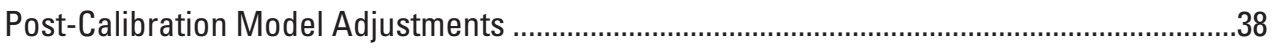

Comparison of Observations and Simulated Equivalents .............................................38

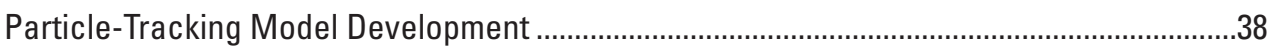

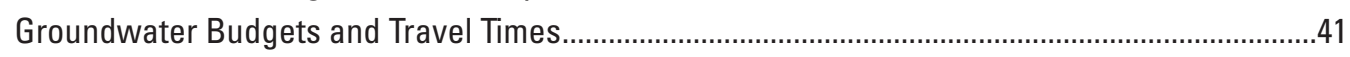

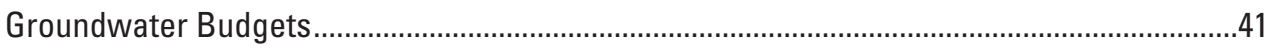

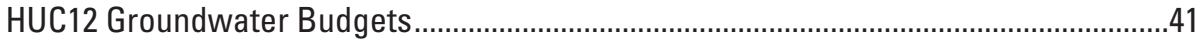

Embayment Groundwater Budgets................................................................................4

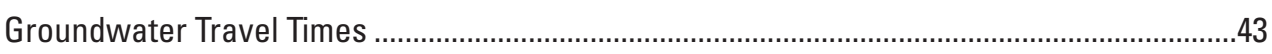

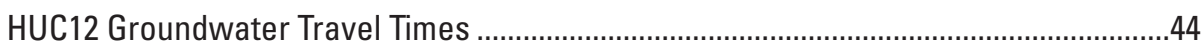

Embayment Groundwater Travel Times .....................................................................46

Contributing Areas of Groundwater Discharge to Coastal and Inland Waters........................48

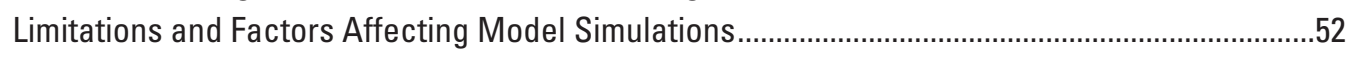

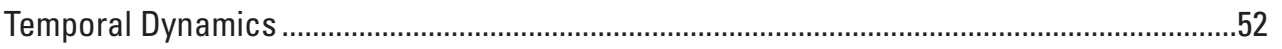




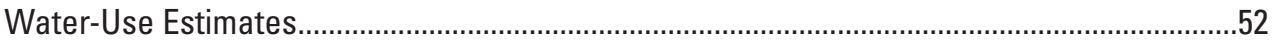

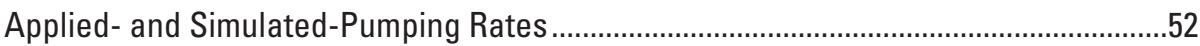

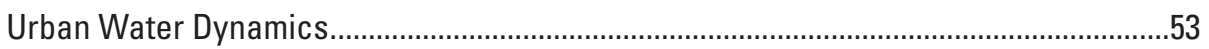

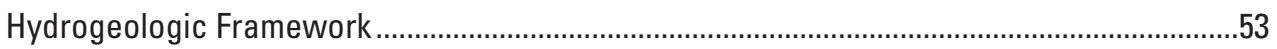

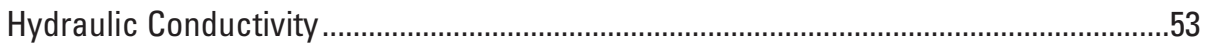

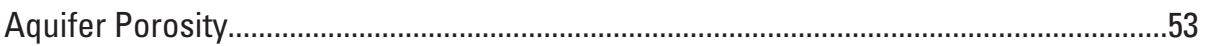

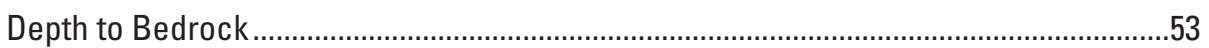

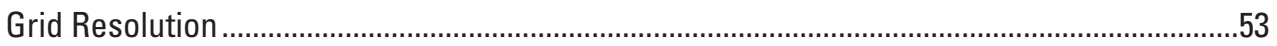

Simulation of Nitrogen Transport by Groundwater Within the Niantic River Watershed ................58

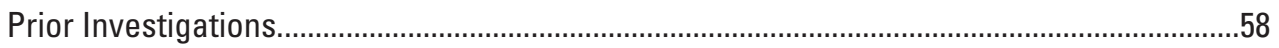

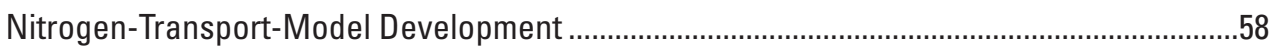

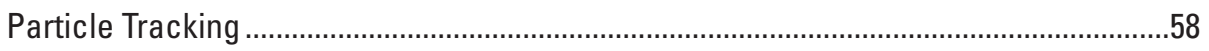

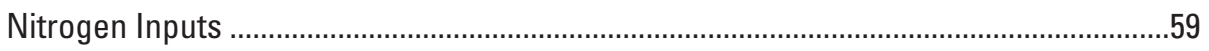

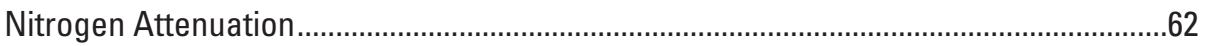

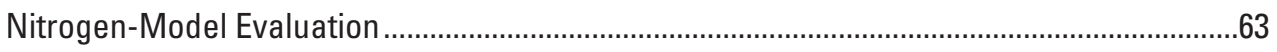

Nitrogen-Transport-Model Results .................................................................................63

Comparison with Nitrogen Loads from Other Studies ......................................................66

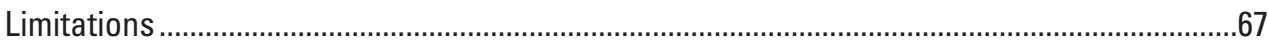

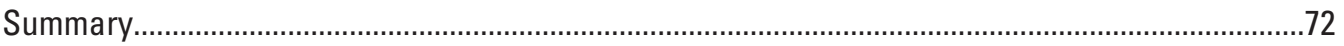

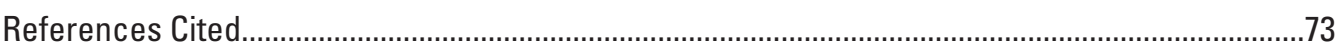

Appendix 1. Comparison of Analysis Periods for Well and Streamgage Data..............................79

Appendix 2. Estimation of Private-Well Withdrawals and Septic Return Flows.............................82

Appendix 3. Estimation of Stream Width ...................................................................................

\section{Figures}

1. Map of the study area along the northern coastline of the Long Island Sound ................3

2. Map of HUC12 watersheds and priority embayments within the study area ....................8

3. Map showing bedrock geology of the north shore of Long Island Sound ........................9

4. Map showing surficial geology of the north shore of Long Island Sound .......................10

5. Map of major streams and rivers on the north shore of Long Island Sound ...................11

6. Map showing unconsolidated surficial-sediment thickness on the north shore of Long Island Sound......................................................................................................13

7. Map showing locations and map numbers of 65 groundwater-monitoring wells and the locations and site numbers of 14 streamgages and their associated watersheds on the north shore of Long Island Sound

8. Map showing locations of the calculated stream water-level altitudes on the north shore of Long Island Sound that were used for model calibration......

9. Map of public water-supply and public sewer service on the north shore of Long Island Sound.

10. Maps of estimated private-well withdrawals, septic return flows, and net groundwater loss from both private wells and septic return flows on the north shore of Long Island Sound

11. Map of groundwater withdrawals for public water supply on the north shore of Long Island Sound 
12. Map of base and extended HUC12 boundaries in a subset of the study area in coastal Connecticut

13. Scatterplot of observed and simulated base-flow values for 14 streamgages on the north shore of Long Island Sound.....

14. Map of estimated natural groundwater-recharge rates on the north shore of Long Island Sound

15. Graphs showing observed and simulated groundwater levels, observed and simulated stream altitudes, and observed and simulated base-flow discharges, north shore of Long Island Sound

16. Maps of groundwater-level residuals and stream-altitude residuals on the north shore of Long Island Sound

17. Map of base-flow-discharge residuals as a percentage of observed base flow on the north shore of Long Island Sound.....

18. Maps of flooded land and dry streams with specified ranges of errors on the north shore of Long Island Sound

19. Maps showing percentages of inflows to the groundwater system for each extended HUC12 watershed from natural recharge and septic return flows on the north shore of Long Island Sound.

20. Maps showing percentages of inflows into and outflows from the groundwater system by lateral transfers of water for each extended HUC12 watershed on the north shore of Long Island Sound.

21. Maps showing percentage of groundwater discharge from each extended HUC12 watershed to inland water and coastal water on the north shore of Long Island Sound

22. Maps showing ratio of groundwater discharge to inland waters to groundwater discharge to coastal waters for each extended HUC12 watershed with groundwater discharge to coastal waters and percentage of groundwater discharge from each extended HUC12 watershed to wells on the north shore of Long Island Sound....

23. Graphs showing cumulative fraction of groundwater discharge by travel time on the north shore of Long Island Sound for groundwater discharge to all waters, inland waters, and coastal waters.

24. Map of groundwater travel times by discharge location (inland waters or coastal waters) on the north shore of Long Island Sound.

25. Map of median groundwater travel-times on the north shore of Long Island Sound by extended HUC12 watershed

26. Graphs showing the effects of varying aquifer porosity on groundwater outflows to coastal waters, inland waters, and wells; and on travel times for groundwater discharge to all waters and to coastal waters, north shore of Long Island Sound.

27. Map of the Niantic River watershed, Connecticut

28. Graphs showing the effects of varying grid-resolution on groundwater outflows to coastal waters, inland waters, and wells; and on travel times for groundwater discharge to all waters and to coastal waters, Niantic River watershed, Connecticut

29. Maps of nitrogen inputs to the groundwater system in the Niantic River watershed, Connecticut

30. Maps of land use in the Niantic River watershed, Connecticut

31. Schematics of the sequential nitrogen attenuation calculations shown in general, and with an example for a particle with nitrogen inputs from fertilizer 
32. Map showing nitrogen yields from selected basin segments in the Niantic River watershed, Connecticut, June 1-3, 2005.

33. Graph showing observed and simulated base-flow nitrogen load in the Niantic River watershed, Connecticut.

34. Graph showing nitrogen inputs and attenuation in the Niantic River watershed, Connecticut..

35. Graphs showing fraction of groundwater nitrogen by discharge location and nitrogen source in the Niantic River watershed, Connecticut.

36. Maps showing simulated rates of nitrogen loading and removal within the Niantic River watershed, Connecticut.

37. Graphs showing simulated groundwater travel time for nitrogen by discharge location within the Niantic River watershed, Connecticut

38. Maps of nitrogen inputs by groundwater travel time within the Niantic River watershed, Connecticut.

\section{Tables}

1. HUC12 watersheds on the north shore of Long Island Sound

2. Priority embayments on the north shore of Long Island Sound .......................................

3. Data sources used in creating the model......................................................................12

4. U.S. Geological Survey groundwater-monitoring wells on the north shore of Long Island Sound used for model calibration...

5. U.S. Geological Survey streamgages on the north shore of Long Island Sound that were used for model calibration

6. Data sources consulted for water-use information.....................................................23

7. Withdrawal rates from wells on the north shore of Long Island Sound .........................24

8. Groundwater-recharge rates by sediment type on the north shore of Long Island Sound.

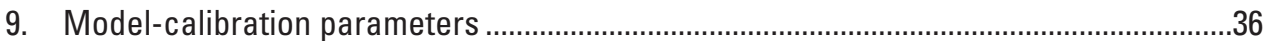

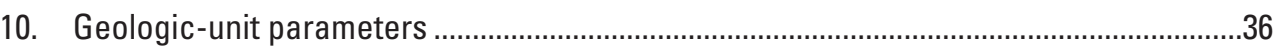

11. Observation groups, weights, and values of the absolute mean residuals .....................37

12. Estimated rates of nitrogen loading, by nitrogen source and land cover, on the north shore of Long Island Sound...

13. Estimated rates of nitrogen attenuation, by nitrogen source, zone, and land cover, on the north shore of Long Island Sound

14. Simulated nitrogen inputs, outputs, and removal rates by discharge location and nitrogen source within the Niantic River watershed, Connecticut. 


\section{Conversion Factors}

U.S. customary units to International System of Units

\begin{tabular}{|c|c|c|}
\hline Multiply & By & To obtain \\
\hline \multicolumn{3}{|c|}{ Length } \\
\hline inch (in.) & 2.54 & centimeter $(\mathrm{cm})$ \\
\hline foot $(\mathrm{ft})$ & 0.3048 & meter $(\mathrm{m})$ \\
\hline mile (mi) & 1.609 & kilometer $(\mathrm{km})$ \\
\hline \multicolumn{3}{|c|}{ Area } \\
\hline acre & 4,047 & square meter $\left(\mathrm{m}^{2}\right)$ \\
\hline acre & 0.004047 & square kilometer $\left(\mathrm{km}^{2}\right)$ \\
\hline square foot $\left(\mathrm{ft}^{2}\right)$ & 0.09290 & square meter $\left(\mathrm{m}^{2}\right)$ \\
\hline square mile $\left(\mathrm{mi}^{2}\right)$ & 2.590 & square kilometer $\left(\mathrm{km}^{2}\right)$ \\
\hline \multicolumn{3}{|c|}{ Volume } \\
\hline gallon (gal) & 0.003785 & cubic meter $\left(\mathrm{m}^{3}\right)$ \\
\hline million gallons (Mgal) & 3,785 & cubic meter $\left(\mathrm{m}^{3}\right)$ \\
\hline cubic foot $\left(\mathrm{ft}^{3}\right)$ & 0.02832 & cubic meter $\left(\mathrm{m}^{3}\right)$ \\
\hline cubic yard $\left(\mathrm{yd}^{3}\right)$ & 0.7646 & cubic meter $\left(\mathrm{m}^{3}\right)$ \\
\hline acre-foot (acre-ft) & 1,233 & cubic meter $\left(\mathrm{m}^{3}\right)$ \\
\hline \multicolumn{3}{|c|}{ Flow rate } \\
\hline cubic foot per day $\left(\mathrm{ft}^{3} / \mathrm{d}\right)$ & 0.02832 & cubic meter per day $\left(\mathrm{m}^{3} / \mathrm{d}\right)$ \\
\hline cubic foot per second $\left(\mathrm{ft}^{3} / \mathrm{s}\right)$ & 2446.576 & cubic meter per day $\left(\mathrm{m}^{3} / \mathrm{d}\right)$ \\
\hline gallon per day (gal/d) & 0.003785 & cubic meter per day $\left(\mathrm{m}^{3} / \mathrm{d}\right)$ \\
\hline \multicolumn{3}{|c|}{ Hydraulic conductivity } \\
\hline foot per day (ft/d) & 0.3048 & meter per day $(\mathrm{m} / \mathrm{d})$ \\
\hline \multicolumn{3}{|c|}{ Leakance } \\
\hline foot per day per foot $([\mathrm{ft} / \mathrm{d}] / \mathrm{ft})$ & 1 & meter per day per meter $([\mathrm{m} / \mathrm{d}] / \mathrm{m})$ \\
\hline \multicolumn{3}{|c|}{ Mass } \\
\hline $\begin{array}{l}\text { pounds per square mile per year }\left[\left(\mathrm{lb} / \mathrm{mi}^{2}\right) /\right. \\
\mathrm{yr}]\end{array}$ & 0.001751 & kilogram per hectare per year $[(\mathrm{kg} / \mathrm{ha}) / \mathrm{yr}]$ \\
\hline \multicolumn{3}{|c|}{ Density } \\
\hline pound per cubic foot $\left(\mathrm{lb} / \mathrm{ft}^{3}\right)$ & 16.02 & kilogram per cubic meter $\left(\mathrm{kg} / \mathrm{m}^{3}\right)$ \\
\hline
\end{tabular}

International System of Units to U.S. customary units

\begin{tabular}{lcl}
\hline \multicolumn{1}{c}{ Multiply } & By & \multicolumn{1}{c}{ To obtain } \\
\hline centimeter $(\mathrm{cm})$ & Length & \\
meter $(\mathrm{m})$ & 0.3937 & inch (in.) \\
kilometer $(\mathrm{km})$ & 3.281 & foot (ft) \\
meter $(\mathrm{m})$ & 0.6214 & mile (mi) \\
\hline & 1.094 & yard (yd) \\
\hline square meter $\left(\mathrm{m}^{2}\right)$ & Area & \\
square kilometer $\left(\mathrm{km}^{2}\right)$ & 0.0002471 & acre \\
square centimeter $\left(\mathrm{cm}^{2}\right)$ & 247.1 & acre \\
square meter $\left(\mathrm{m}^{2}\right)$ & 0.001076 & square foot $\left(\mathrm{ft}^{2}\right)$ \\
square kilometer $\left(\mathrm{km}^{2}\right)$ & 10.76 & square foot $\left(\mathrm{ft}^{2}\right)$ \\
& 0.3861 & square mile $\left(\mathrm{mi}^{2}\right)$
\end{tabular}




\begin{tabular}{|c|c|c|}
\hline Multiply & By & To obtain \\
\hline \multicolumn{3}{|c|}{ Volume } \\
\hline cubic meter $\left(\mathrm{m}^{3}\right)$ & 264.2 & gallon (gal) \\
\hline cubic meter $\left(\mathrm{m}^{3}\right)$ & 0.0002642 & million gallons (Mgal) \\
\hline cubic meter $\left(\mathrm{m}^{3}\right)$ & 35.31 & cubic foot $\left(\mathrm{ft}^{3}\right)$ \\
\hline cubic meter $\left(\mathrm{m}^{3}\right)$ & 1.308 & cubic yard $\left(\mathrm{yd}^{3}\right)$ \\
\hline cubic meter $\left(\mathrm{m}^{3}\right)$ & 0.0008107 & acre-foot (acre-ft) \\
\hline \multicolumn{3}{|c|}{ Flow rate } \\
\hline meter per day $(\mathrm{m} / \mathrm{d})$ & 3.281 & foot per day $(\mathrm{ft} / \mathrm{d})$ \\
\hline meter per year $(\mathrm{m} / \mathrm{yr})$ & 3.281 & foot per year ft/yr) \\
\hline cubic meter per day $\left(\mathrm{m}^{3} / \mathrm{d}\right)$ & 35.31 & cubic foot per day $\left(\mathrm{ft}^{3} / \mathrm{d}\right)$ \\
\hline cubic meter per day $\left(\mathrm{m}^{3} / \mathrm{d}\right)$ & 0.0004087 & cubic foot per second $\left(\mathrm{ft}^{3} / \mathrm{s}\right)$ \\
\hline cubic meter per day $\left(\mathrm{m}^{3} / \mathrm{d}\right)$ & 264.2 & gallon per day (gal/d) \\
\hline \multicolumn{3}{|c|}{ Hydraulic conductivity } \\
\hline meter per day $(\mathrm{m} / \mathrm{d})$ & 3.281 & foot per day (ft/d) \\
\hline \multicolumn{3}{|c|}{ Leakance } \\
\hline meter per day per meter $([\mathrm{m} / \mathrm{d}] / \mathrm{m})$ & 1 & foot per day per foot $([\mathrm{ft} / \mathrm{d}] / \mathrm{ft})$ \\
\hline \multicolumn{3}{|c|}{ Mass } \\
\hline kilogram per hectare per year $[(\mathrm{kg} / \mathrm{ha}) / \mathrm{yr}]$ & 571.1 & $\begin{array}{l}\text { pounds per square mile per year }[(\mathrm{lb} / \\
\left.\left.\mathrm{mi}^{2}\right) / \mathrm{yr}\right]\end{array}$ \\
\hline \multicolumn{3}{|c|}{ Density } \\
\hline kilogram per cubic meter $\left(\mathrm{kg} / \mathrm{m}^{3}\right)$ & 0.06242 & pound per cubic foot $\left(\mathrm{lb} / \mathrm{ft}^{3}\right)$ \\
\hline
\end{tabular}

\section{Datum}

Vertical coordinate information is referenced to the North American Vertical Datum of 1988 (NAVD 88).

Horizontal coordinate information is referenced to the North American Datum of 1983 (NAD 83).

Altitude, as used in this report, refers to distance above the vertical datum.

\section{Abbreviations}

CT DEEP Connecticut Department of Energy and Environmental Protection

EPA U.S. Environmental Protection Agency

LIS Long Island Sound

LISS Long Island Sound Study

NLCD National Land Cover Database (published in 2011)

SWB Soil Water Balance (model of the glaciated United States in 2018)

USGS U.S. Geological Survey 


\title{
Simulation of Groundwater Budgets and Travel Times for Watersheds on the North Shore of Long Island Sound, With Implications for Nitrogen-Transport Studies
}

\author{
By Janet R. Barclay and John R. Mullaney
}

\section{Abstract}

Aquatic systems in and around the Long Island Sound (LIS) provide a variety of ecological and economic benefits, but in some areas of the LIS, aquatic ecosystems have become degraded by excess nitrogen. A substantial fraction of the nitrogen inputs to the LIS are transported through the groundwater-flow system. Because groundwater travel times in surficial aquifers can exceed 100 years, multiyear lags are introduced between inputs at the water table in recharge areas and discharge to inland or coastal receiving waters. The U.S. Geological Survey, in cooperation with the Connecticut Department of Energy and Environmental Protection and the U.S. Environmental Protection Agency's Long Island Sound Study, developed a steady-state groundwater model of the watersheds draining from the northern shore of the LIS for the purpose of calculating groundwater budgets and travel times to coastal waters.

The model was developed by using the MODFLOWNWT software and existing spatial data on aquifers, river networks, land-surface altitudes, land cover, groundwater recharge, and water use. Coastal waters were delineated on the basis of the National Wetland Inventory; all non-coastal waters were collectively termed "inland waters." A coarse-resolution model was calibrated by using the PEST++ software, longterm records of water levels in 65 wells, stream altitudes from 477 streams, base-flow records for 14 streamgages that are relatively unaffected by withdrawals, and error metrics based on incorrectly simulated flooding and incorrectly simulated dry streams. The calibrated values were used in a fine-resolution model in which the mean absolute residuals were 4.5 meters for groundwater levels, 1.3 meters for stream altitudes, and 7,200 cubic meters per day (2.9 cubic feet per second) for base flow. About 89 percent of the terrestrial cells were correctly simulated with the water table below land surface, and nearly 90 percent of the cells representing streams were correctly simulated as having the water table above the stream bottom. Together, these metrics suggest that this model is robust for simulating regional-scale groundwater patterns.
Simulated groundwater budgets were compiled for the entire study area, for each HUC12 (Hydrologic Unit Code no. 12) watershed and its adjacent coastal waters, if applicable, within the study area, and for 14 coastal-embayment watersheds. Most groundwater (90.6 percent of inflows) discharged to inland waters, with smaller fractions to coastal waters ( 7.0 percent) and well withdrawals ( 2.4 percent). When computed for HUC12 watersheds with coastal discharge, the portions of groundwater discharging to coastal waters ranged from 0.02 to 66 percent of groundwater outflows, with a median of 13 percent. Within priority-embayment watersheds, the portions of groundwater discharging to coastal waters ranged from 2 to 56 percent, with a median of 15 percent.

Groundwater travel times also were simulated for the entire study area, for each HUC12 watershed and its adjacent coastal waters, if applicable, within the study area and for 14 priority coastal embayments. Within the entire study area, the median groundwater travel time was 1.9 years, with an interquartile range of 0.1 to 5.9 years. Sensitivity analysis of groundwater travel times within a subbasin in the study area indicates that the travel times are a function of the grid resolution, with coarser grids resulting in shorter median travel times. Travel times for groundwater discharging to coastal waters were similar to travel times for groundwater discharging to inland waters, with a median of 1.9 years. Median travel times for the HUC12 watersheds ranged from 0.9 to 53.5 years, with a median of 1.8 years. Among HUC12 watersheds that include coastal areas, travel times for groundwater discharging to coastal waters ranged from less than 1 to 61.6 years, with a median of 2.8 years. The HUC12 watersheds with the longest simulated travel times were in the urban area near New York City where the model performance is less accurate. Median travel times for groundwater discharging to coastal waters within the priority-embayment watersheds ranged from less than 1 to 18.6 years, with a median of 2.3 years.

A more focused analysis was conducted for the Niantic River watershed to demonstrate the applicability of the regional model to local-scale nitrogen-transport analyses by using nitrogen-input and -attenuation rates from literature sources. Nitrogen inputs were estimated by using land-coverbased loading factors, and attenuation was estimated by using 
attenuation factors based on geologic zones and soil properties. Based on this analysis, groundwater transports an estimated 22,000 kilograms of nitrogen per year (2.9 kilograms of nitrogen per hectare per year) to streams, rivers, and coastal waters within the Niantic River watershed. Approximately 36 percent of discharging nitrogen is from atmosphericdeposition sources, 38 percent is from fertilizers, and 26 percent is from septic systems. Most of the groundwatertransported nitrogen ( 88 percent) discharges first to streams and rivers, with only 12 percent discharging directly to coastal waters. Travel times for groundwater-transported nitrogen ranged from less than 1 day to more than 100 years, with a median of 1.6 years.

\section{Introduction}

Aquatic systems in and around the Long Island Sound (LIS) provide a variety of ecological and economic benefits such as flood and storm protection, water filtration, recreation, habitat for fish and bird populations (important for both commerce and recreation), and carbon sequestration (Long Island Sound Study [LISS], 2015). In some areas of the LIS and in many embayments along the Connecticut coastline, aquatic ecosystems have become degraded by excess nitrogen from sources such as wastewater-treatment plants, septic systems, and fertilizer (LISS, 2015). A substantial fraction of total nitrogen inputs to the LIS is transported through the groundwaterflow system. Some of the nitrogen is discharged from the groundwater-flow system into streams and rivers, which then flow into the LIS, and some of the nitrogen is discharged directly into the LIS from the groundwater-flow system. Consequently, understanding the magnitude and spatial distribution of groundwater discharge is essential to planning and evaluating conservation-management efforts aimed at reducing nitrogen inputs to the LIS.

The time required for water to travel through the groundwater system from recharge to discharge also provides important information about the hydrologic system. Referred to as groundwater-residence times or groundwater lags, groundwater travel times provide insight into how water moves through an aquifer system, which in turn affects the transport and attenuation of nutrients and other contaminants (McGuire and McDonnell, 2006). Groundwater travel times in some surficial aquifers can exceed 100 years (Sanford and others, 2012) and thus introduce substantial time lags between inputs at the water table and discharge to receiving waters. Currently, the groundwater-flow system on the north shore of the LIS (fig. 1) is not well understood. In particular, groundwater budgets, travel times, and discharge receptors have not been quantified, thus hindering water-resources-management efforts focused on groundwater-transported nitrogen.

To better understand the groundwater-flow system on the north shore of the LIS, the U.S. Geological Survey (USGS), in cooperation with the U.S. Environmental Protection Agency (EPA), Long Island Sound Study (LISS), and Connecticut
Department of Energy and Environmental Protection (CT DEEP), began a study in 2018 to characterize groundwater flow in the watersheds draining into the LIS along the Connecticut coast and adjacent areas of New York and Rhode Island (fig. 1). A groundwater model was developed to provide a regional tool to evaluate the groundwater-flow systems in these watersheds. In this study, the model was used as a framework for understanding the groundwater component of flow to surface water in the HUC12 watersheds (Hydrologic Unit Code no. 12) (Seaber and others, 1987) within the study area (fig. 2, table 1) and to a subset of coastal embayments (fig. 2, table 2) along the north shore of LIS. In the future, the model could be used for more detailed assessments of priority areas (for example, groundwater-discharge rates, travel times, and nitrogen loading to coastal embayments in primary-tier watersheds). An additional analysis was conducted to demonstrate the use of the regional model for quantifying spatial patterns of nitrogen loading and attenuation and for determining groundwater travel times at the HUC12 watershed scale. The Niantic River watershed in eastern Connecticut (fig. 2C) was chosen for this analysis.

\section{Purpose and Scope}

The purpose of this report is to document the analysis of hydrologic budgets and groundwater travel times within the watersheds of the north shore of the LIS. The report also describes the development and calibration of a groundwaterflow model of the watersheds draining the north shore of LIS, as well as a case study that demonstrates the use of the model to understand groundwater transport of nitrogen within the Niantic River watershed. The report describes the (1) development and calibration of the groundwater-flow model, (2) travel times and the volumetric components of the groundwater budgets in the watersheds draining into the LIS, (3) travel times and the volumetric components of the groundwater budgets in the watersheds that drain into a selected subset of LIS embayments, and (4) estimations of the groundwater nitrogen loading, attenuation, and transport times within the Niantic River watershed.

\section{Hydrogeology}

On the north shore of the LIS, unconsolidated glacial sediments that include both glacial till and stratified sediments overlie bedrock. Principal aquifers include valley-fill glacial aquifers and both crystalline and sandstone bedrock aquifers (Olcott, 1995).

\section{Geologic Setting}

Bedrock on the north shore of the LIS is predominantly metamorphic in origin, except in the Connecticut River Valley, where it is sedimentary, and in Rhode Island, where there are areas of igneous, and mixed metamorphic 


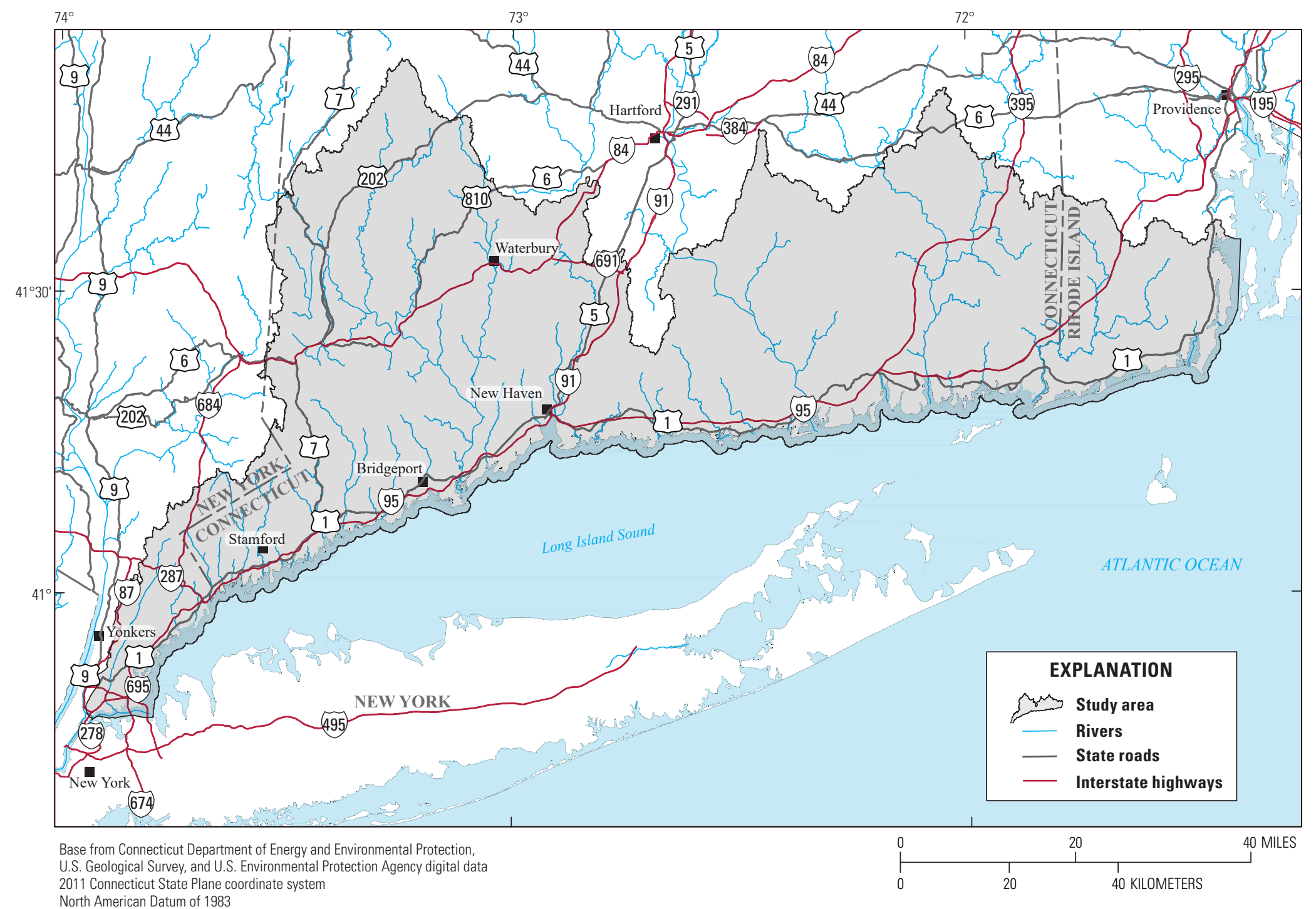

Figure 1. The study area along the northern coastline of the Long Island Sound (coastal Connecticut and adjacent areas of New York and Rhode Island). 
Table 1. HUC12 watersheds on the north shore of Long Island Sound (coastal Connecticut and adjacent areas of New York and Rhode Island).

[Map no. refers to number on figure 2. Base area is the area within the study area of each HUC12 watershed. Extended area includes adjacent coastal waters and was used for the calculation of groundwater budgets and travel times. Area is based on the modeled area and may differ slightly from mapped areas due to grid discretization. Area is reported in square kilometers; "--" indicates that the boundary was not extended; * indicates that part of the HUC12 watershed is outside of the study area]

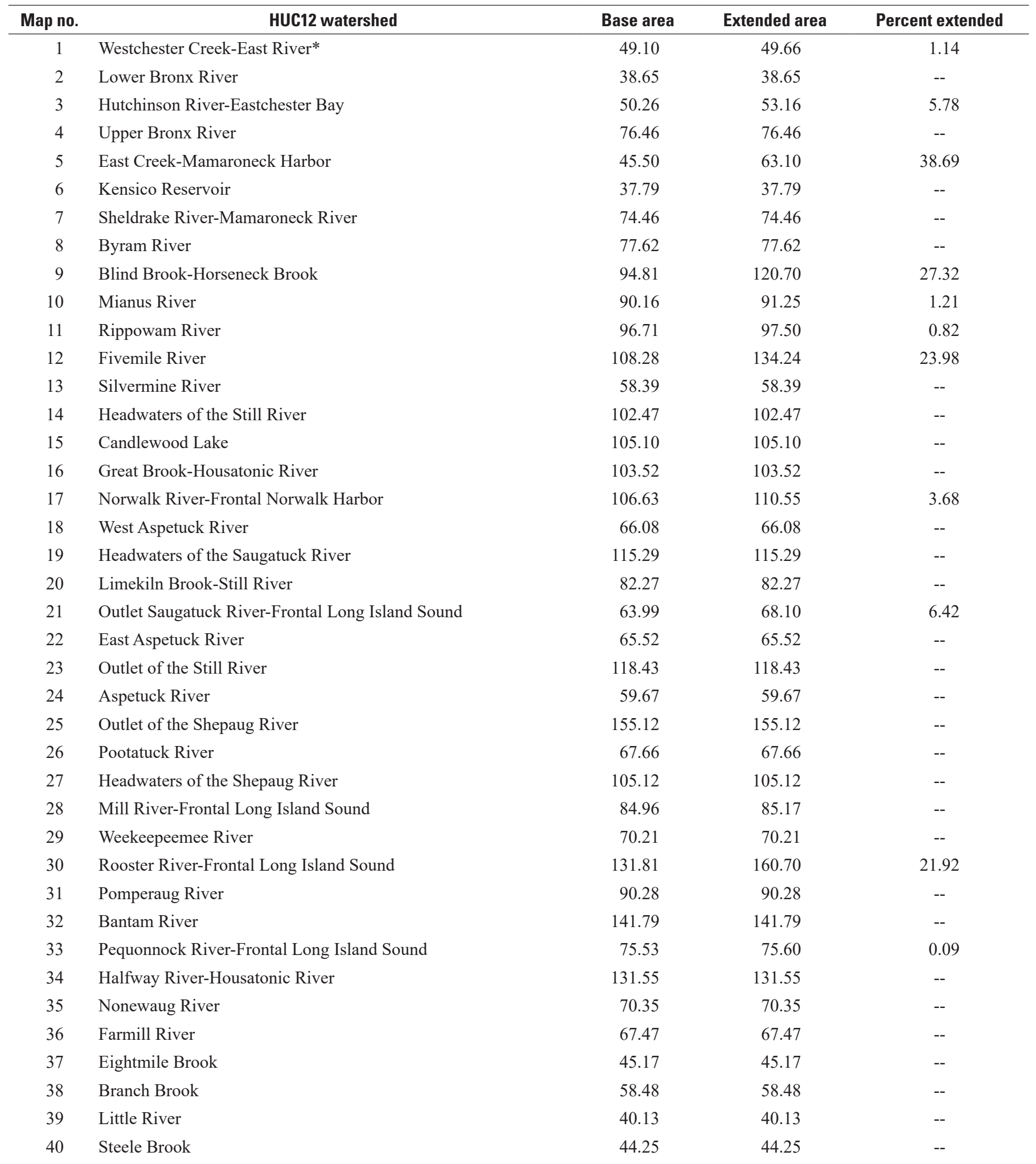


Table 1. HUC12 watersheds on the north shore of Long Island Sound (coastal Connecticut and adjacent areas of New York and Rhode Island).-Continued

[Map no. refers to number on figure 2. Base area is the area within the study area of each HUC12 watershed. Extended area includes adjacent coastal waters and was used for the calculation of groundwater budgets and travel times. Area is based on the modeled area and may differ slightly from mapped areas due to grid discretization. Area is reported in square kilometers; "--" indicates that the boundary was not extended; * indicates that part of the HUC12 watershed is outside of the study area]

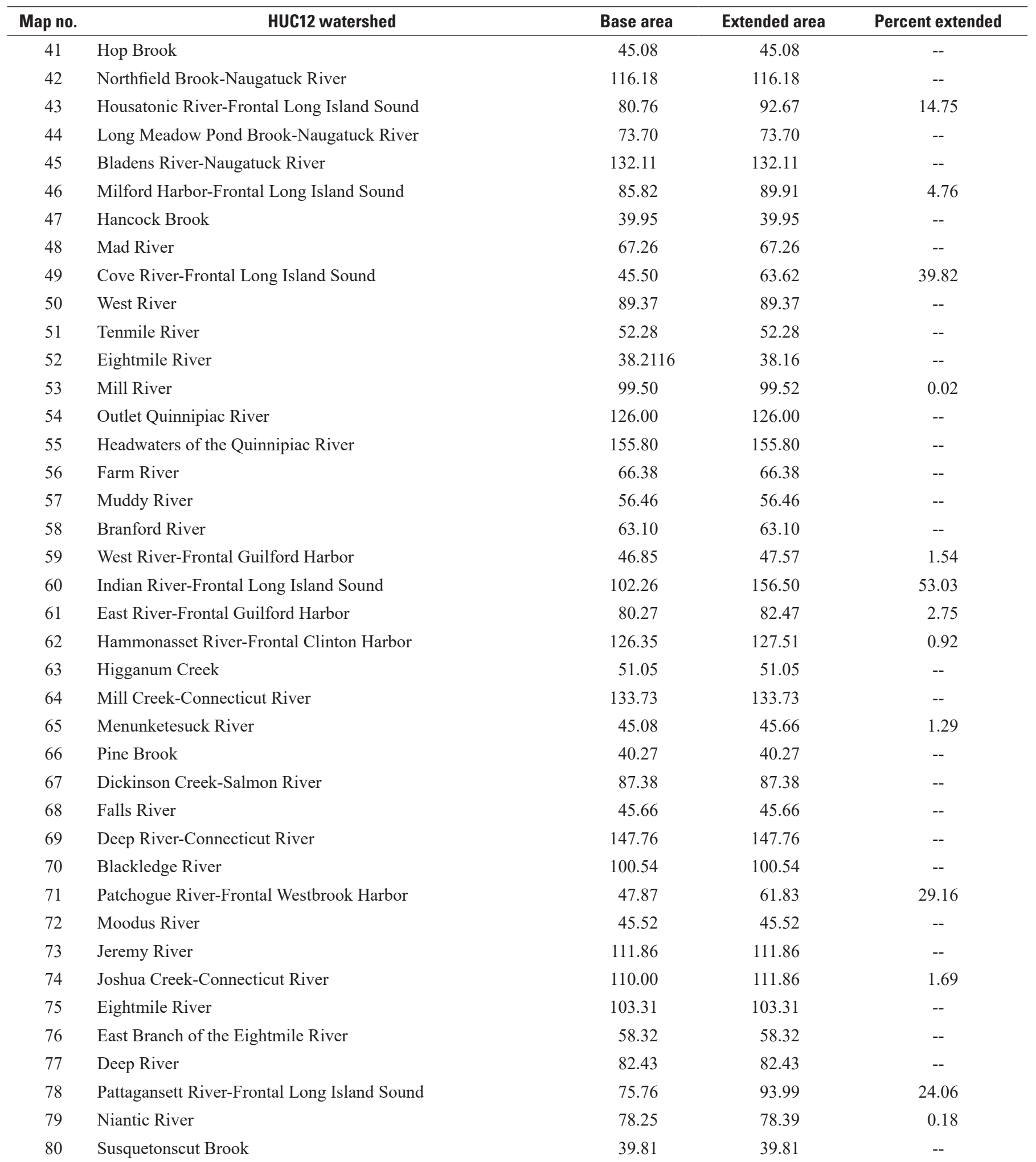


Table 1. HUC12 watersheds on the north shore of Long Island Sound (coastal Connecticut and adjacent areas of New York and Rhode Island).-Continued

[Map no. refers to number on figure 2. Base area is the area within the study area of each HUC12 watershed. Extended area includes adjacent coastal waters and was used for the calculation of groundwater budgets and travel times. Area is based on the modeled area and may differ slightly from mapped areas due to grid discretization. Area is reported in square kilometers; "--" indicates that the boundary was not extended; * indicates that part of the HUC12 watershed is outside of the study area]

\begin{tabular}{|c|c|c|c|c|}
\hline Map no. & HUC12 watershed & Base area & Extended area & Percent extended \\
\hline 81 & Pease Brook-Yantic River & 130.71 & 130.71 & -- \\
\hline 82 & Stony Brook & 57.14 & 57.14 & -- \\
\hline 83 & Fenger Brook-Frontal Long Island Sound & 43.25 & 55.21 & 27.66 \\
\hline 84 & Beaver Brook-Shetucket River & 157.66 & 157.66 & -- \\
\hline 85 & Thames River-Frontal New London Harbor & 148.46 & 154.54 & 4.10 \\
\hline 86 & Merrick Brook & 54.14 & 54.14 & -- \\
\hline 87 & Little River & 112.13 & 112.13 & -- \\
\hline 88 & Poquonock River-Frontal Fishers Island Sound & 43.06 & 44.13 & 2.48 \\
\hline 89 & Poquetanuck Brook & 73.56 & 73.56 & -- \\
\hline 90 & Cory Brook-Quinebaug River & 139.19 & 139.19 & -- \\
\hline 91 & Mystic River-Frontal Fishers Island Sound & 69.58 & 69.72 & 0.20 \\
\hline 92 & Broad Brook & 42.36 & 42.36 & -- \\
\hline 93 & Stony Brook-Frontal Fishers Island Sound & 113.13 & 144.67 & 27.88 \\
\hline 94 & Lower Pachaug River & 64.75 & 64.75 & -- \\
\hline 95 & Mill Brook & 46.17 & 46.17 & -- \\
\hline 96 & Shunock River & 42.94 & 42.94 & -- \\
\hline 97 & Upper Pachaug River & 98.78 & 98.78 & -- \\
\hline 98 & Lower Pawcatuck River & 41.13 & 41.55 & 1.02 \\
\hline 99 & Ashaway River & 72.07 & 72.07 & -- \\
\hline 100 & Lower Wood River & 73.95 & 73.95 & -- \\
\hline 101 & Upper Wood River & 158.31 & 158.31 & -- \\
\hline 102 & Tomaquag Brook-Pawcatuck River & 147.74 & 147.74 & -- \\
\hline 103 & Ninigret Pond-Frontal Block Island Sound & 73.14 & 121.80 & 66.53 \\
\hline 104 & Beaver River & 32.14 & 32.14 & -- \\
\hline 105 & Usquepaug River-Pawcatuck River & 54.88 & 54.88 & -- \\
\hline 106 & Usquepaug River & 94.13 & 94.13 & -- \\
\hline 107 & Chipuxet River-Pawcatuck River & 66.59 & 66.59 & -- \\
\hline 108 & Point Judith Pond-Frontal Block Island Sound & 28.71 & 40.76 & 41.99 \\
\hline 109 & Saugatucket River & 44.45 & 44.45 & -- \\
\hline 110 & Pettaquamscutt River-Frontal Atlantic Ocean & 43.87 & 62.13 & 41.61 \\
\hline 111 & Lower West Passage* & 90.46 & 91.42 & 1.05 \\
\hline
\end{tabular}

and sedimentary bedrock (fig. 3). The metamorphic bedrock is older (Precambrian through Devonian) than the sedimentary bedrock (Mesozoic in the Connecticut River Valley and Carboniferous in coastal Rhode Island) (Olcott, 1995).

Unconsolidated glacial sediments overlie the bedrock along the north shore of the LIS (fig. 4). These sediments were deposited during periods of glacial movement between 75,000 and 11,000 years ago. Glacial till, which consists of unsorted and unstratified sediments of various grain sizes, was deposited under the glaciers throughout the area. In some areas, glacial-till deposits remain from an earlier period of glaciation between 190,000 and 130,000 years ago. Glacial stratified sediments were deposited by glacial meltwater during glacial melting or stagnation and are predominantly found in stream valleys, whereas glacial till is predominantly present at the surface in upland areas or beneath stratified sediments in the valleys. The unconsolidated glacial sediments overlying bedrock range in thickness from less than 1 meter $(\mathrm{m})$ to 
Table 2. Priority embayments on the north shore of Long Island Sound (coastal Connecticut and adjacent areas of New York and Rhode Island).

[Priority embayments were identified by the Connecticut Department of Energy and Environmental Protection (2019a). Map letter refers to the letter on figure 2. Areas are reported in square kilometers. All locations are in Connecticut (CT)]

\begin{tabular}{clcc}
\hline Map letter & \multicolumn{1}{c}{ Embayment } & Embayment area & Watershed area \\
\hline a & Norwalk Harbor, CT & 2.53 & 108.93 \\
b & Saugatuck River, CT & 2.50 & 66.03 \\
c & Sasco Brook, CT & 0.05 & 26.52 \\
d & Mill River, CT & 0.26 & 85.07 \\
e & Farm River, CT & 0.42 & 67.31 \\
f & Pages Cove, CT & 0.07 & 0.71 \\
g & Niantic River, CT & 3.21 & 78.32 \\
h & Beebee Cove, CT & 0.53 & 2.35 \\
i & Mystic River, CT & 1.17 & 69.53 \\
j & Williams Cove, CT & 0.06 & 4.89 \\
k & Quanaduck Cove, CT & 0.19 & 1.98 \\
l & Wequetequock Cove, CT & 0.82 & 33.86 \\
m & Little Narragansett Bay, CT & 3.31 & 9.18 \\
$\mathrm{n}$ & Pawcatuck River, CT & 2.64 & 41.06 \\
\hline
\end{tabular}

more than $100 \mathrm{~m}$ with a mean of $9.8 \mathrm{~m}$ across the north shore of the LIS (Melvin and others, 1992; Olcott, 1995; Yager and others, 2018a).

\section{Hydrologic Setting}

The aquifer system consists of bedrock, glacial, and alluvial aquifers. Crystalline-rock aquifers are within the metamorphic bedrock in the study area (fig. 3), and the Connecticut River valley contains a Mesozoic-bedrock basin aquifer (arkose, a type of sedimentary bedrock) (U.S. Geological Survey, 2003). Private residential wells and many smaller public-supply wells typically pump from bedrock aquifers. Unconsolidated surficial valley-fill aquifers of glacial and alluvial origin are found in glacial stratified sediments along river valleys (fig. 4) across the region (U.S. Geological Survey, 2002). Major public-supply wells are often completed in the valley-fill aquifers because of their high transmissivity and proximity to large rivers.

The primary source of water to the aquifer system is precipitation recharge. From 1981 to 2010, precipitation ranged from 109.3 to 142.0 centimeters per year $(\mathrm{cm} / \mathrm{yr})$, with a mean of $125.7 \mathrm{~cm} / \mathrm{yr}$, across the north shore of the LIS (PRISM Climate Group, 2012). Region-wide, approximately
39.5 percent of precipitation ( 0 to $114.4 \mathrm{~cm} / \mathrm{yr}$ with a mean of $49.6 \mathrm{~cm} / \mathrm{yr}$ ) enters the groundwater system as recharge (Yager and others, 2018a).

Groundwater discharges to both fresh and saline surface water. More than 7,000 kilometers $(\mathrm{km})$ of stream length, including reaches of the Housatonic, Connecticut, and Thames Rivers, cover the model area, with a slightly higher density of larger streams and rivers in the western part of the study area than in the eastern part (fig. 5). Groundwater discharge is an important source of water, nutrients, and habitat in many of these streams and rivers. Fresh groundwater also discharges to saline surface water in estuaries, embayments, and the open Long Island Sound (fig. 2). The magnitude of groundwater discharge to fresh and saline waters cannot be measured directly but will be calculated as part of this regional-modeling analysis.

Groundwater levels vary spatially and temporally across the north shore of LIS. Groundwater levels tend to be shallower in flat areas near water bodies, and deeper in areas with locally higher altitudes. The depth to water below land surface varies from $0 \mathrm{~m}$ near wetlands to more than $10 \mathrm{~m}$ in upland areas. Groundwater levels generally are lowest during the late summer and early fall when evapotranspiration rates typically exceed precipitation rates. Groundwater is typically withdrawn by public-water suppliers, residents with private wells, and a variety of industries (Dieter and others, 2018). 


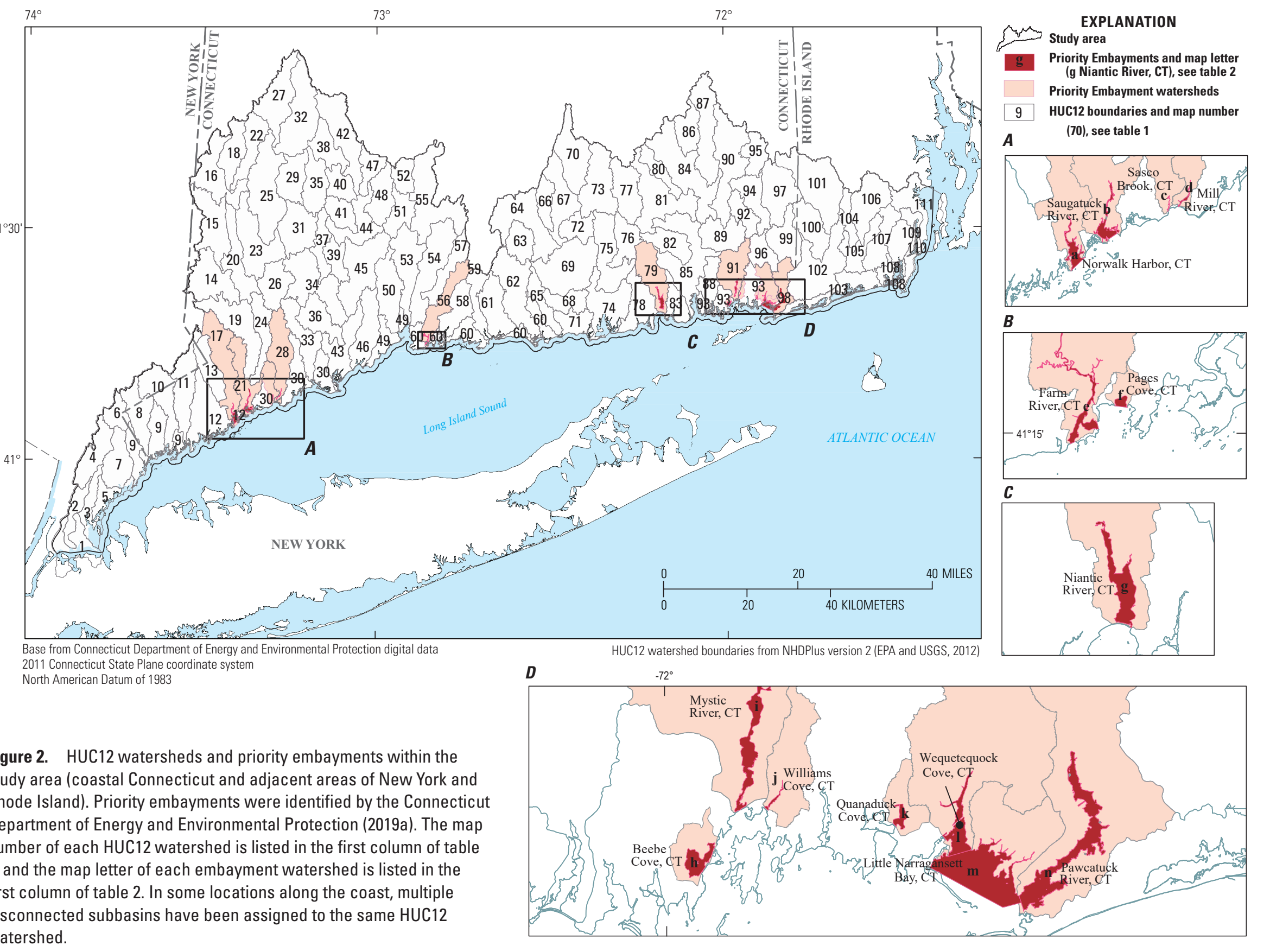




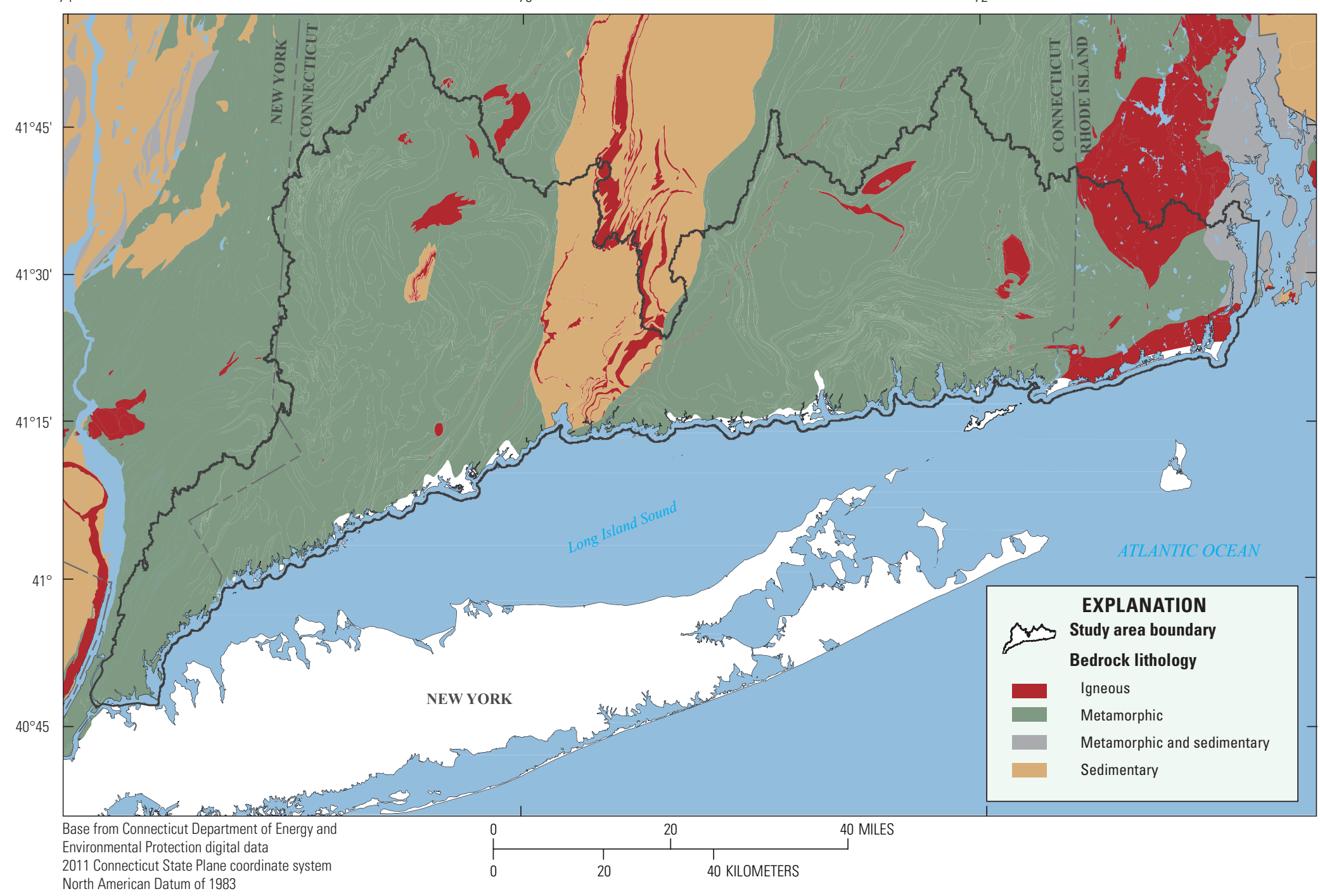

Figure 3. Bedrock geology of the north shore of Long Island Sound (coastal Connecticut and adjacent areas of New York and Rhode Island). Geology from Horton (2017). 


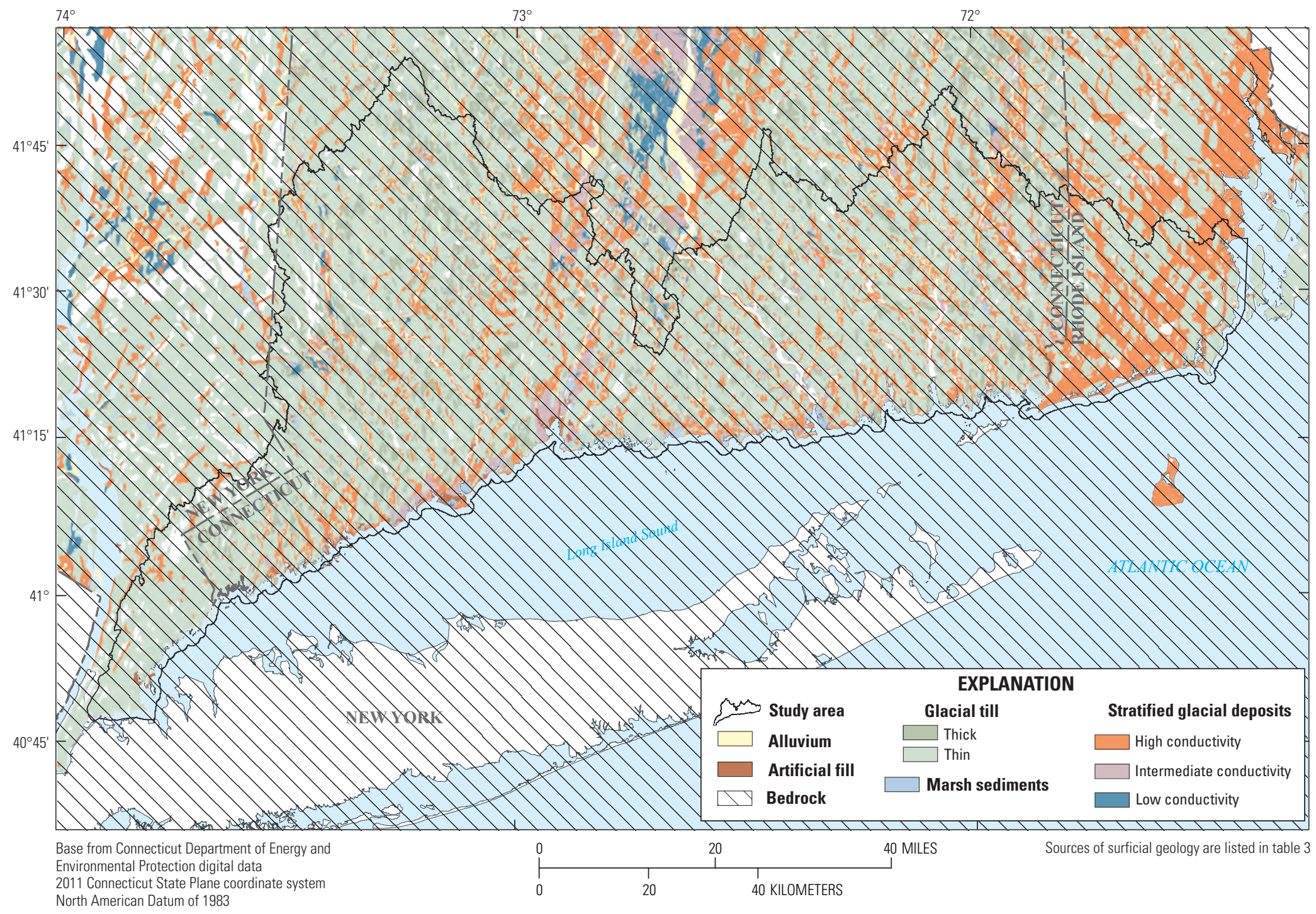

Figure 4. Surficial geology of the north shore of Long Island Sound (coastal Connecticut and adjacent areas of New York and Rhode Island). 


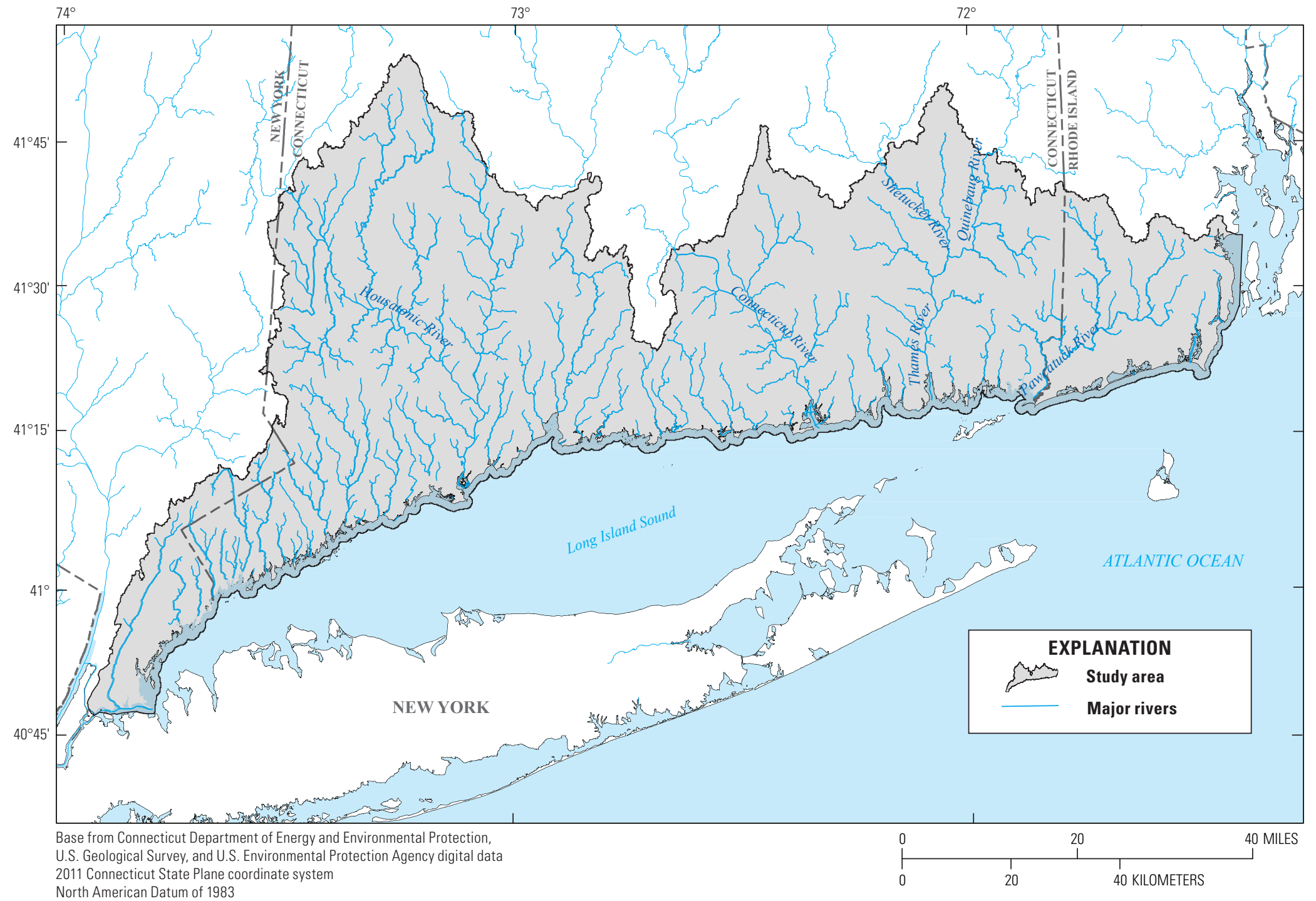

Figure 5. Major streams and rivers on the north shore of Long Island Sound (coastal Connecticut and adjacent areas of New York and Rhode Island). 


\section{Data Compilation and Analysis}

Existing lithologic, hydrologic, land-use, and wateruse data were compiled from a variety of national and local sources (table 3). Lithologic, land-use, water-use, and some hydrologic data (groundwater recharge) were used as inputs to the model. The remaining hydrologic data (groundwater levels and streamflows) were used as observations in the modelcalibration process.

\section{Lithologic Data}

Information about sediment distribution, thickness, and rock type across the north shore of the LIS was compiled from State and national data sources (table 3 ). The bedrock altitude was calculated by subtracting the thickness of the unconsolidated glacial sediments (Long Island Sound Resource Center and U.S. Geological Survey, 2004; Yager and others, 2018a) (fig. 6) from the land surface altitude (U.S. Environmental Protection Agency and U.S. Geological Survey, 2012). Bedrock lithology was simplified into four types: igneous, metamorphic, sedimentary, and interspersed metamorphic and sedimentary. Surficial-material lithology was simplified into eight types: alluvium, artificial fill (of unknown lithology), stratified glacial deposits - high hydraulic conductivity, stratified glacial deposits - intermediate hydraulic conductivity, stratified glacial deposits- - low hydraulic conductivity, marsh sediments, thick glacial till, and thin glacial till. The "stratified glacial deposits - high conductivity" type encompasses sediments ranging from fine sand to gravel and that may include interspersed fine sand, silt, and clay. Stratified glacial deposits of high conductivity are mapped as "Coarse Deposits" or "Stacked Coarse Deposits" in Connecticut (Stone and others, 1992); as "Beach," "Barrier Island," Lacustrine delta,"
"Lacustrine sand," "Outwash sand and gravel," "Fluvial sand and gravel," "Kame deposits," and "Kame moraine" in New York (New York State Museum and New York State Geological Survey, 1999); and as "Mixed" and "Outwash" in Rhode Island (RIGIS, 1989). The "stratified glacial deposits of intermediate conductivity" encompasses a combined unit of coarser sediments overlying finer sediments. Stratified glacial deposits of intermediate conductivity were mapped only for Connecticut and identified as "Stacked Coarse Deposits Overlying Fine Deposits" (Stone and others, 1992). Stratified glacial deposits of low conductivity include very fine sand, silt, and clay, which are mapped as "Fine Deposits" in Connecticut (Stone and others, 1992), and as "Lacustrine silt and clay" in New York (New York State Museum and New York State Geological Survey, 1999). There are no mapped areas of stratified glacial deposits of low conductivity in Rhode Island. Thin glacial till, sometimes referred to as surface till, is at the land surface and was deposited during the late Wisconsin glaciation ( 75,000 to 11,000 years ago) (Melvin and others, 1992). Thick glacial till, which is alternatively referred to as drumlin till, is thicker, deeper, and more compact than thin glacial till and was deposited during the late Illinois glaciation (180,000 to 150,000 years ago). Because the water table frequently is located within bedrock in areas of thin glacial till, this till and the underlying bedrock were represented in the model as a till-bedrock complex.

\section{Hydrologic Data}

Initial values of groundwater recharge were based on a Soil Water Balance (SWB) model (Westenbroek and others, 2010) developed for the glacial-aquifer system (Yager and others, 2018b). The SWB model calculates groundwater recharge by using a modified Thornthwaite-Mather water

Table 3. Data sources used in creating the model.

\begin{tabular}{lc}
\hline \multicolumn{1}{c}{ Variable } & Source \\
\hline Land-Surface Altitude & National Elevation Dataset (U.S. Environmental Protection Agency and U.S. Geological \\
& Survey, 2012) \\
Surficial-Sediment Thickness & $\begin{array}{c}\text { Digital products from a hydrogeologic framework for Quaternary sediments within the } \\
\text { glaciated conterminous United States (Yager and others, 2018a); Connecticut Glacial }\end{array}$ \\
& Sediment Thickness (Long Island Sound Resource Center and U.S. Geological Survey, \\
& 2004) \\
Surficial Sediment_-New York & Surficial Geology_-Lower Hudson Sheet (New York State Museum and New York State \\
& Geological Survey, 1999) \\
Surficial Sediment_-Connecticut & Surficial Materials Map of Connecticut (Stone and others, 1992) and Quaternary Geologic \\
& Map of Connecticut and Long Island Sound Basin (Stone and others, 2005) digitized \\
& in Connecticut Quaternary Geology and Surficial Materials Polygon (U.S. Geological \\
Survey and others, 2005) & Rhode Island Glacial Deposits (Rhode Island Geographic Information System, 1989) \\
Surficial Sediment-Rhode Island & State Geologic Map Compilation (Horton, 2017) \\
Bedrock Geology & Digital products from a hydrogeologic framework for Quaternary sediments within the \\
groundwater Recharge & glaciated conterminous United States (Yager and others, 2018a) \\
\hline
\end{tabular}




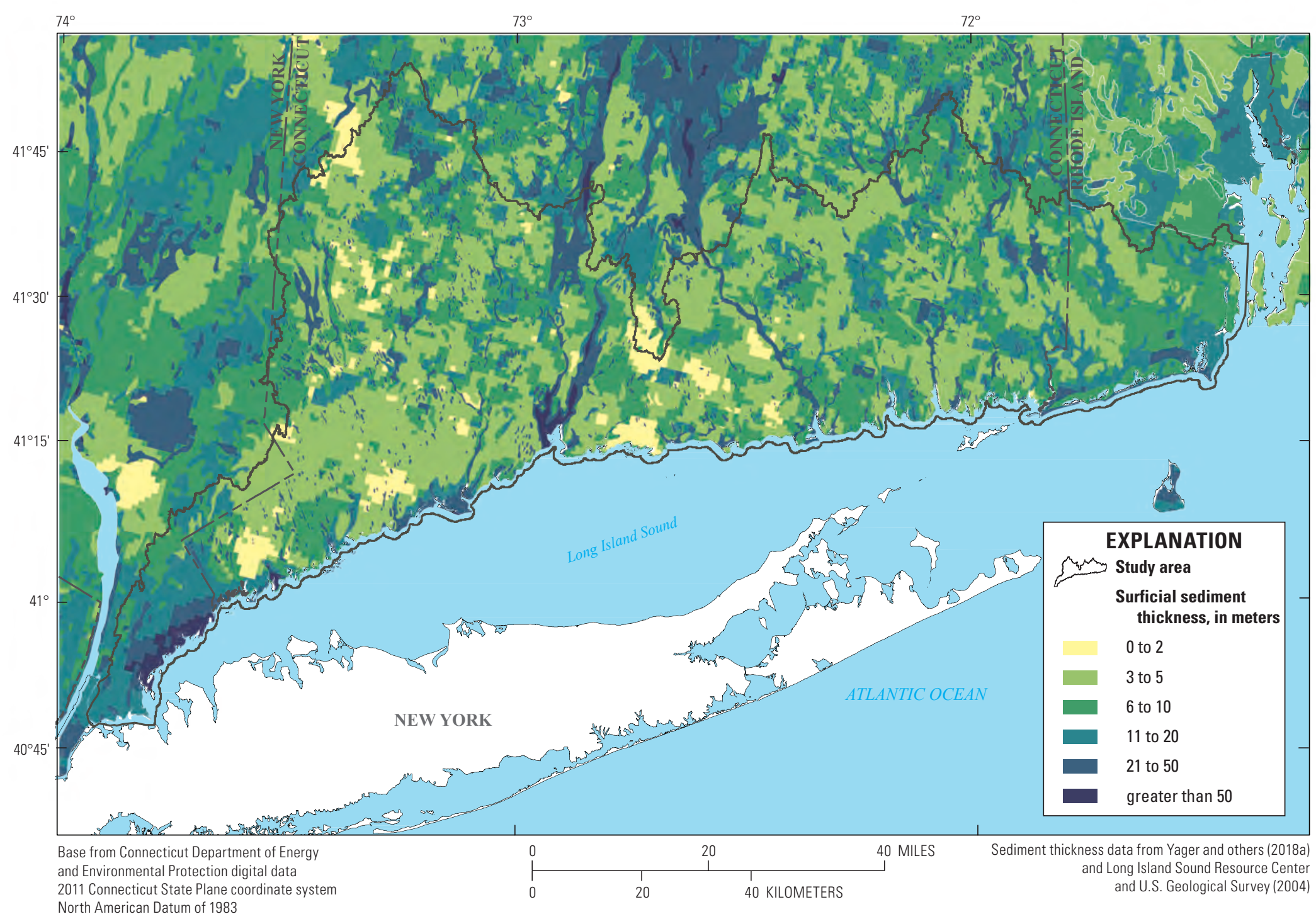

Figure 6. Unconsolidated surficial-sediment thickness on the north shore of Long Island Sound (coastal Connecticut and adjacent areas of New York and Rhode Island). 
balance and data on precipitation, air temperature, land use, and soil properties. Initial estimates of groundwater recharge were modified during the model-calibration process.

Four groups of data were used to calibrate the groundwater-flow model: mean groundwater levels from 65 wells (U.S. Geological Survey, 2020), mean streamflows during base-flow conditions from 14 gages (U.S. Geological Survey, 2020), stream water-level altitudes from 477 stream segments mapped in the NHDPlus version 2 dataset (U.S. Environmental Protection Agency and U.S. Geological Survey, 2012), and the general observation that the altitudes of groundwater levels are typically below the land surface in terrestrial areas (the absence of flooded land) and at or above the water-level altitudes of perennial water bodies (the absence of dry streams). Three of these observation groupsgroundwater levels, stream water-level altitudes, and flooded land or dry streams - provide important information about water levels. The fourth group - stream base flow-provides important information about the movement of water through the subsurface, including the amount of water entering the system as recharge. Data for two of the observations, stream water-level altitudes and flooded land or dry streams, were generated from maps of long-term, steady-state conditions (U.S. Environmental Protection Agency and U.S. Geological Survey, 2012). The other two observations, mean groundwater levels and mean stream base flow, were calculated by using available measurements from water years 1990 through 2019 (U.S. Geological Survey, 2020). Data from wells and streamgages with longer periods of record were analyzed to confirm that the mean values based on data from wells and streamgages with shorter periods of record were consistent with long term averages. The details of the analysis are given in appendix 1.

Wells were selected on the basis of their period of record and number of measurements: water levels in all wells used in the analysis had been measured 50 or more times during water years 1990 through 2019. To account for different measurement frequencies for each selected well, available mean waterlevel data from water years 1990 through 2019 was aggregated first by year and month, then year, and finally by the total time period. In total, water levels from 65 wells were used for model calibration (fig. 7, table 4). Water-level data for all wells are available in the National Water Information System (U.S. Geological Survey, 2020).

Flow records for streamgages at sites unaffected by water withdrawals, such as groundwater pumping, surfacewater diversions, and regulation, were selected for the baseflow observations. The streamgages within the study area designated as "Ref" in the Geospatial Attributes of Gages for Evaluating Streamflow, version II (GAGESII) dataset (Falcone, 2011) and two additional streamgages (USGS site numbers 01208873 and 012035055 ) were selected. Mean base flow at each streamgage was calculated by using two algorithms, PART (Rutledge, 1998) and BFI (Gustard and others, 1992), as implemented in the DVstats package in the statistical software R (Lorenz, 2017; R Core Team, 2019).
The PART and BFI algorithms were selected because they use different approaches to determining percentages of streamflow originating as base flow rather than stormflow; the average of the two values was defined as the base-flow observation at the 14 streamgages used for model calibration (fig. 7, table 5). Streamflow data for all gages are available in the National Water Information System (U.S. Geological Survey, 2020).

Stream water-level altitudes were used as indicators of the water table. Stream reaches were selected on the basis of land-surface slope from model cells with a slope of no more than 0.5 percent because, in flat areas, the stream water-level altitude is more likely to be representative of the nearby water table. The altitudes for the selected reaches were calculated from the NHDPlus dataset (U.S. Environmental Protection Agency and U.S. Geological Survey, 2012) by assuming a linear decrease in altitude over the segment length. In total, 477 stream water-level-altitude observations were used (fig. 8) (Barclay and Mullaney, 2021b).

\section{Land-Use Data}

Land cover throughout the north shore of the LIS varies from heavily forested to heavily populated. Deciduous forest was the dominant land cover (45 percent of the study area), and development of all densities composed the second most abundant land cover (27 percent of the study area) in 2011 (Homer and others, 2015). The mean population density in 2010 was 434 people per square kilometer $\left(\mathrm{km}^{2}\right)$, with densities greater than 3,000 people per $\mathrm{km}^{2}$ near the urban areas of New Haven and Stamford, Conn., and Port Chester, N.Y.; and densities greater than 10,000 people per $\mathrm{km}^{2}$ near New York City, N.Y. (Falcone, 2016) (fig. 1). The combination of forested land cover and high population densities outside city centers results in a largely exurban landscape. In exurban areas, private wells and private septic systems become important components of the groundwater budget due to relatively higher populations than in rural areas, but limited public water infrastructure as compared to urban areas.

In this study, land-use data from the 2011 National Land Cover Database (NLCD) (Homer and others, 2015) were used to identify areas of open water and wetlands for the analysis of flooded land and dry rivers, to adjust groundwater recharge by pervious cover, and to estimate rates of nitrogen loading for the nitrogen-transport case study. Land-use data were also used in the estimation of groundwater recharge used as an input in this study (Yager and others, 2018b).

\section{Water-Use Data}

Water use was estimated for public water-supply wells, private residential wells, and septic return flows. Withdrawals for industrial, thermoelectric, and agricultural purposes, as well as non-community public water-supply wells in Connecticut, were not included in this analysis because of limited data availability and estimated small magnitudes 
Table 4. U.S. Geological Survey groundwater-monitoring wells on the north shore of Long Island Sound (coastal Connecticut and adjacent areas of New York and Rhode Island) used for model calibration.

[Map no. refers to the number on figure 7. Altitude is in meters relative to NAVD 88. "Water level" is defined as the mean water-level altitude in meters for the period of analysis relative to NAVD 88. Data for monitoring wells are available in the National Water Information System (U.S. Geological Survey, 2020). CT, Connecticut; RI, Rhode Island]

\begin{tabular}{|c|c|c|c|c|c|c|c|c|}
\hline $\begin{array}{l}\text { Map } \\
\text { no. }\end{array}$ & Site number & Station name & $\begin{array}{l}\text { Decimal } \\
\text { latitude }\end{array}$ & $\begin{array}{l}\text { Decimal } \\
\text { longitude }\end{array}$ & $\begin{array}{l}\text { Land-surface } \\
\text { altitude }\end{array}$ & $\begin{array}{l}\text { Mean water- } \\
\text { level altitude }\end{array}$ & $\begin{array}{c}\text { Number of } \\
\text { measure- } \\
\text { ments }\end{array}$ & Period of analysis \\
\hline 1 & 405329073514901 & B 71.1 & 40.89154119 & -73.863221 & 87.00 & 58.07 & 69 & $\begin{array}{l}08 / 15 / 2006 \text { through } \\
04 / 14 / 2015\end{array}$ \\
\hline 2 & 410443073414101 & CT-GW 22 & 41.07788889 & -73.67752778 & 216.54 & 211.11 & 218 & $\begin{array}{l}10 / 05 / 2001 \text { through } \\
11 / 18 / 2020\end{array}$ \\
\hline 3 & 410515073415901 & CT-GW 23 & 41.0874722 & -73.6831667 & 365.94 & 339.38 & 216 & $\begin{array}{l}10 / 05 / 2001 \text { through } \\
11 / 18 / 2020\end{array}$ \\
\hline 4 & 410628073413301 & CT-GW 21 GREENWICH, CT & 41.10788889 & -73.69238889 & 465.11 & 436.28 & 234 & $\begin{array}{l}10 / 05 / 2001 \text { through } \\
10 / 29 / 2020\end{array}$ \\
\hline 5 & 411058073182001 & CT-FF 33 & 41.18255 & -73.3049194 & 154.03 & 148.81 & 341 & $\begin{array}{l}08 / 19 / 1993 \text { through } \\
11 / 18 / 2020\end{array}$ \\
\hline 6 & 411103073181301 & CT-FF 32 & 41.18368889 & -73.3033222 & 175.96 & 168.35 & 321 & $\begin{array}{l}\text { 08/19/1993 through } \\
11 / 18 / 2020\end{array}$ \\
\hline 7 & 411118073175801 & CT-FF 31 & 41.18849167 & -73.2990056 & 272.47 & 264.60 & 337 & $\begin{array}{l}10 / 26 / 1993 \text { through } \\
11 / 18 / 2020\end{array}$ \\
\hline 8 & 411124073172201 & CT-FF 30 & 41.1902111 & -73.28900278 & 335.76 & 330.87 & 337 & $\begin{array}{l}08 / 19 / 1993 \text { through } \\
11 / 18 / 2020\end{array}$ \\
\hline 9 & 411256073153101 & CT-FF 23 & 41.21565185 & -73.258171 & 113.27 & 105.47 & 428 & $\begin{array}{l}\text { 09/26/1966 through } \\
11 / 18 / 2020\end{array}$ \\
\hline 10 & 411723072344701 & CT-MA 314 & 41.289821 & -72.5792586 & 45.00 & 27.09 & 145 & $\begin{array}{l}03 / 29 / 1982 \text { through } \\
05 / 28 / 1996\end{array}$ \\
\hline 11 & 411735072315001 & CT-CL 225 & 41.29315485 & -72.53009039 & 30.59 & 24.39 & 378 & $\begin{array}{l}12 / 18 / 1991 \text { through } \\
11 / 19 / 2020\end{array}$ \\
\hline 12 & 411826072322401 & CT-CL 224 & 41.30715278 & -72.53995278 & 154.19 & 133.67 & 364 & $\begin{array}{l}10 / 22 / 1991 \text { through } \\
09 / 24 / 2020\end{array}$ \\
\hline 13 & 411832072325501 & CT-CL 223 CLINTON, CT & 41.30890556 & -72.5480694 & 204.48 & 198.94 & 378 & $\begin{array}{l}10 / 22 / 1991 \text { through } \\
10 / 22 / 2020\end{array}$ \\
\hline 14 & 412013072030601 & CT-GT 19 & 41.33681667 & -72.0512806 & 18.81 & 3.67 & 1186 & $\begin{array}{l}05 / 01 / 1958 \text { through } \\
11 / 19 / 2020\end{array}$ \\
\hline 15 & 412154071462901 & RI-WEW 522 WESTERLY, RI & 41.36509999 & -71.7742336 & 44.06 & 31.59 & 304 & $\begin{array}{l}04 / 20 / 1966 \text { through } \\
10 / 28 / 2020\end{array}$ \\
\hline
\end{tabular}


Table 4. U.S. Geological Survey groundwater-monitoring wells on the north shore of Long Island Sound (coastal Connecticut and adjacent areas of New York and Rhode Island) used for model calibration.-Continued

[Map no. refers to the number on figure 7. Altitude is in meters relative to NAVD 88. "Water level" is defined as the mean water-level altitude in meters for the period of analysis relative to NAVD 88. Data for monitoring wells are available in the National Water Information System (U.S. Geological Survey, 2020). CT, Connecticut; RI, Rhode Island]

\begin{tabular}{|c|c|c|c|c|c|c|c|c|}
\hline $\begin{array}{c}\text { Map } \\
\text { no. }\end{array}$ & Site number & Station name & $\begin{array}{l}\text { Decimal } \\
\text { latitude }\end{array}$ & $\begin{array}{l}\text { Decimal } \\
\text { longitude }\end{array}$ & $\begin{array}{l}\text { Land-surface } \\
\text { altitude }\end{array}$ & $\begin{array}{l}\text { Mean water- } \\
\text { level altitude }\end{array}$ & $\begin{array}{c}\text { Number of } \\
\text { measure- } \\
\text { ments }\end{array}$ & Period of analysis \\
\hline 16 & 412214071394001 & RI-CHW 18 & 41.37065647 & -71.66061838 & 26.00 & 7.48 & 369 & $\begin{array}{l}10 / 01 / 1946 \text { through } \\
10 / 29 / 2020\end{array}$ \\
\hline 17 & 412307072515201 & CT-NHV 201 & 41.3853861 & -72.8637333 & 25.77 & 9.83 & 419 & $\begin{array}{l}09 / 22 / 1975 \text { through } \\
11 / 18 / 2020\end{array}$ \\
\hline 18 & 412417072541901 & CT-HM 449 & 41.4049611 & -72.90445 & 230.68 & 213.66 & 334 & $\begin{array}{l}01 / 07 / 1993 \text { through } \\
11 / 18 / 2020\end{array}$ \\
\hline 19 & 412417072541902 & CT-HM 450 & 41.4049583 & -72.90442778 & 231.63 & 219.45 & 295 & $\begin{array}{l}01 / 07 / 1993 \text { through } \\
11 / 18 / 2020\end{array}$ \\
\hline 20 & 412423072542801 & CT-HM 445 & 41.4066111 & -72.9074583 & 188.02 & 164.43 & 332 & $\begin{array}{l}07 / 12 / 1988 \text { through } \\
11 / 18 / 2020\end{array}$ \\
\hline 21 & 412424071423601 & RI-CHW 587 & 41.4067669 & -71.70950979 & 90.00 & 80.71 & 262 & $\begin{array}{l}\text { 10/08/1992 through } \\
11 / 26 / 2013\end{array}$ \\
\hline 22 & 412429073165101 & CT-NT 15, NEWTOWN, CT & 41.40820556 & -73.28055 & 261.28 & 255.68 & 443 & $\begin{array}{l}12 / 01 / 1966 \text { through } \\
10 / 29 / 2020\end{array}$ \\
\hline 23 & 412434071422401 & RI-CHW 586 & 41.40954469 & -71.70617638 & 125.00 & 120.14 & 269 & $\begin{array}{l}09 / 30 / 1992 \text { through } \\
11 / 26 / 2013\end{array}$ \\
\hline 24 & 412541072542001 & CT-HM 448 & 41.42786389 & -72.90500278 & 109.38 & 96.16 & 335 & $\begin{array}{l}01 / 07 / 1993 \text { through } \\
11 / 18 / 2020\end{array}$ \\
\hline 25 & 412546072541701 & CT-HM 447 & 41.4294972 & -72.904125 & 99.07 & 96.36 & 336 & $\begin{array}{l}01 / 07 / 1993 \text { through } \\
11 / 18 / 2020\end{array}$ \\
\hline 26 & 412546072541702 & CT-HM 446 & 41.42951944 & -72.9041194 & 99.05 & 95.71 & 335 & $\begin{array}{l}04 / 14 / 1993 \text { through } \\
11 / 18 / 2020\end{array}$ \\
\hline 27 & 412550072510701 & CT-NHV 202 & 41.4306528 & -72.85148937 & 55.00 & 4.94 & 188 & $\begin{array}{c}10 / 07 / 1975 \text { through } \\
10 / 29 / 2001\end{array}$ \\
\hline 28 & 412718071415201 & RI-RIW 785 & 41.4551 & -71.697288 & 85.00 & 60.02 & 357 & $\begin{array}{c}10 / 30 / 1989 \text { through } \\
10 / 29 / 2020\end{array}$ \\
\hline 29 & 412746071510601 & CT-NSN 78 & 41.49626667 & -71.8508306 & 314.22 & 309.84 & 401 & $\begin{array}{c}10 / 25 / 1991 \text { through } \\
11 / 19 / 2020\end{array}$ \\
\hline 30 & 412824072173301 & CT-SM 7 & 41.47357778 & -72.29161389 & 233.48 & 223.30 & 420 & $\begin{array}{l}\text { 03/19/1979 through } \\
11 / 19 / 2020\end{array}$ \\
\hline
\end{tabular}


Table 4. U.S. Geological Survey groundwater-monitoring wells on the north shore of Long Island Sound (coastal Connecticut and adjacent areas of New York and Rhode Island) used for model calibration.-Continued

[Map no. refers to the number on figure 7. Altitude is in meters relative to NAVD 88. "Water level" is defined as the mean water-level altitude in meters for the period of analysis relative to NAVD 88. Data for monitoring wells are available in the National Water Information System (U.S. Geological Survey, 2020). CT, Connecticut; RI, Rhode Island]

\begin{tabular}{|c|c|c|c|c|c|c|c|c|}
\hline $\begin{array}{l}\text { Map } \\
\text { no. }\end{array}$ & Site number & Station name & $\begin{array}{l}\text { Decimal } \\
\text { latitude }\end{array}$ & $\begin{array}{l}\text { Decimal } \\
\text { longitude }\end{array}$ & $\begin{array}{l}\text { Land-surface } \\
\text { altitude }\end{array}$ & $\begin{array}{l}\text { Mean water- } \\
\text { level altitude }\end{array}$ & $\begin{array}{c}\text { Number of } \\
\text { measure- } \\
\text { ments }\end{array}$ & Period of analysis \\
\hline 31 & 412844071422802 & RI-RIW 600 RICHMOND, RI & 41.47898869 & -71.7072888 & 99.27 & 65.42 & 329 & $\begin{array}{l}\text { 09/21/1977 through } \\
10 / 28 / 2020\end{array}$ \\
\hline 32 & 412916073121701 & CT-SB 42 SOUTHBURY, CT & 41.48787368 & -73.2042801 & 468.71 & 453.54 & 388 & $\begin{array}{l}08 / 19 / 1993 \text { through } \\
10 / 30 / 2020\end{array}$ \\
\hline 33 & 412918071321001 & $\begin{array}{l}\text { RI-SNW } 6 \text { SOUTH KINGS- } \\
\text { TOWN, RI }\end{array}$ & 41.48843449 & -71.5356159 & 110.99 & 99.13 & 314 & $\begin{array}{l}11 / 01 / 1947 \text { through } \\
10 / 28 / 2020\end{array}$ \\
\hline 34 & 412923071361601 & RI-SNW 515 & 41.4898228 & -71.6039518 & 127.93 & 99.94 & 133 & $\begin{array}{l}03 / 29 / 1955 \text { through } \\
06 / 21 / 2000\end{array}$ \\
\hline 35 & 412931071514201 & CT-NSN 77 & 41.4921 & -71.85985278 & 516.93 & 504.90 & 391 & $\begin{array}{l}10 / 25 / 1991 \text { through } \\
11 / 19 / 2020\end{array}$ \\
\hline 36 & 412932071374302 & RI-RIW 417 RICHMOND, RI & 41.49232259 & -71.6281194 & 114.67 & 107.82 & 307 & $\begin{array}{l}12 / 22 / 1975 \text { through } \\
10 / 28 / 2020\end{array}$ \\
\hline 37 & 412935071355701 & RI-SNW 1198 & 41.49315616 & -71.59867389 & 112.37 & 102.93 & 303 & $\begin{array}{l}12 / 01 / 1988 \text { through } \\
10 / 29 / 2020\end{array}$ \\
\hline 38 & 412935073122701 & CT-SB 41 & 41.49290556 & -73.2070056 & 379.76 & 331.35 & 513 & $\begin{array}{l}\text { 10/24/1991 through } \\
10 / 30 / 2020\end{array}$ \\
\hline 39 & 412954073125201 & CT-SB 30 & 41.4982472 & -73.2138361 & 255.56 & 236.42 & 547 & $\begin{array}{l}01 / 02 / 1979 \text { through } \\
10 / 30 / 2020\end{array}$ \\
\hline 40 & 413002073131001 & CT-SB 39 & 41.50061667 & -73.21836389 & 190.40 & 184.00 & 553 & $\begin{array}{l}10 / 24 / 1991 \text { through } \\
10 / 30 / 2020\end{array}$ \\
\hline 41 & 413007073250501 & CT-BD 8 & 41.50198056 & -73.4173417 & 255.42 & 225.12 & 413 & $\begin{array}{l}12 / 19 / 1966 \text { through } \\
11 / 18 / 2020\end{array}$ \\
\hline 42 & 413126071455501 & RI-HOW 67 & 41.5239881 & -71.76479119 & 335.00 & 317.46 & 352 & $\begin{array}{l}08 / 30 / 1953 \text { through } \\
10 / 29 / 2020\end{array}$ \\
\hline 43 & 413134073021701 & CT-WB 93 & 41.52611389 & -73.03746389 & 321.80 & 294.62 & 423 & $\begin{array}{l}10 / 01 / 1943 \text { through } \\
10 / 30 / 2020\end{array}$ \\
\hline 44 & 413135071314201 & RI-EXW 278 & 41.5264899 & -71.52783819 & 230.90 & 217.06 & 324 & $\begin{array}{l}08 / 12 / 1954 \text { through } \\
08 / 26 / 2020\end{array}$ \\
\hline 45 & 413148071281601 & RI-NKW 255 & 41.53010139 & -71.4706136 & 50.00 & 40.72 & 384 & $\begin{array}{l}\text { 08/01/1954 through } \\
10 / 29 / 2020\end{array}$ \\
\hline
\end{tabular}


Table 4. U.S. Geological Survey groundwater-monitoring wells on the north shore of Long Island Sound (coastal Connecticut and adjacent areas of New York and Rhode Island) used for model calibration.-Continued

[Map no. refers to the number on figure 7. Altitude is in meters relative to NAVD 88. "Water level" is defined as the mean water-level altitude in meters for the period of analysis relative to NAVD 88. Data for monitoring wells are available in the National Water Information System (U.S. Geological Survey, 2020). CT, Connecticut; RI, Rhode Island]

\begin{tabular}{|c|c|c|c|c|c|c|c|c|}
\hline $\begin{array}{l}\text { Map } \\
\text { no. }\end{array}$ & Site number & Station name & $\begin{array}{l}\text { Decimal } \\
\text { latitude }\end{array}$ & $\begin{array}{l}\text { Decimal } \\
\text { longitude }\end{array}$ & $\begin{array}{l}\text { Land-surface } \\
\text { altitude }\end{array}$ & $\begin{array}{l}\text { Mean water- } \\
\text { level altitude }\end{array}$ & $\begin{array}{c}\text { Number of } \\
\text { measure- } \\
\text { ments }\end{array}$ & Period of analysis \\
\hline 46 & 413202073122401 & CT-WY 1 & 41.534125 & -73.2057611 & 271.07 & 246.95 & 584 & $\begin{array}{l}10 / 04 / 1913 \text { through } \\
10 / 30 / 2020\end{array}$ \\
\hline 47 & 413245072584201 & CT-WB 198 & 41.54643056 & -72.9778222 & 541.98 & 526.32 & 427 & $\begin{array}{l}10 / 01 / 1943 \text { through } \\
10 / 30 / 2020\end{array}$ \\
\hline 48 & 413252071323601 & RI-EXW 554 EXETER, RI & 41.5478786 & -71.5428388 & 156.03 & 146.22 & 327 & $\begin{array}{l}12 / 06 / 1988 \text { through } \\
10 / 28 / 2020\end{array}$ \\
\hline 49 & 413254072335501 & CT-MT 261 & 41.5482861 & -72.5648833 & 150.00 & 127.91 & 407 & $\begin{array}{l}04 / 01 / 1956 \text { through } \\
11 / 19 / 2020\end{array}$ \\
\hline 50 & 413358071433801 & RI-EXW 475 EXETER, RI & 41.56621059 & -71.72673449 & 142.06 & 127.59 & 304 & $\begin{array}{l}03 / 11 / 1981 \text { through } \\
11 / 04 / 2020\end{array}$ \\
\hline 51 & 413400071363101 & RI-EXW 238 & 41.56676699 & -71.608119 & 333.80 & 321.10 & 352 & $\begin{array}{l}\text { 07/27/1954 through } \\
10 / 29 / 2020\end{array}$ \\
\hline 52 & 413423071431901 & RI-EXW 6 & 41.57315507 & -71.7214565 & 132.80 & 126.34 & 302 & $\begin{array}{l}06 / 26 / 1946 \text { through } \\
11 / 27 / 2013\end{array}$ \\
\hline 53 & 413457072252201 & CT-CO 335 & 41.5828833 & -72.4220861 & 149.09 & 141.95 & 412 & $\begin{array}{l}01 / 10 / 1986 \text { through } \\
11 / 19 / 2020\end{array}$ \\
\hline 54 & 413505071452801 & RI-EXW 158 & 41.5848215 & -71.7572911 & 315.00 & 304.10 & 277 & $\begin{array}{l}\text { 09/14/1953 through } \\
06 / 27 / 2014\end{array}$ \\
\hline 55 & 413518072264501 & CT-MB 36 & 41.58843237 & -72.4453635 & 490.30 & 485.65 & 340 & $\begin{array}{l}\text { 08/18/1993 through } \\
11 / 19 / 2020\end{array}$ \\
\hline 56 & 413535072253701 & $\begin{array}{l}\text { CT-MB } 32 \text { MARLBOROUGH, } \\
\text { CT }\end{array}$ & 41.5931333 & -72.4264222 & 257.60 & 252.42 & 421 & $\begin{array}{l}01 / 10 / 1986 \text { through } \\
11 / 19 / 2020\end{array}$ \\
\hline 57 & 413554072270201 & CT-MB 35 & 41.5984324 & -72.4500858 & 515.00 & 502.82 & 339 & $\begin{array}{l}\text { 08/18/1993 through } \\
11 / 19 / 2020\end{array}$ \\
\hline 58 & 413645071332901 & RI-WGW 206 & 41.6126005 & -71.5575618 & 374.00 & 368.49 & 362 & $\begin{array}{l}\text { 10/14/1954 through } \\
08 / 26 / 2020\end{array}$ \\
\hline 59 & 413907071465001 & RI-WGW 181 & 41.65204364 & -71.7800697 & 380.00 & 363.76 & 370 & $\begin{array}{l}\text { 08/02/1966 through } \\
10 / 29 / 2020\end{array}$ \\
\hline 60 & 414054071552001 & CT-PL 1 & 41.681325 & -71.9222583 & 184.51 & 154.84 & 423 & $\begin{array}{l}10 / 01 / 1942 \text { through } \\
11 / 20 / 2020\end{array}$ \\
\hline
\end{tabular}


Table 4. U.S. Geological Survey groundwater-monitoring wells on the north shore of Long Island Sound (coastal Connecticut and adjacent areas of New York and Rhode Island) used for model calibration.-Continued

[Map no. refers to the number on figure 7. Altitude is in meters relative to NAVD 88. "Water level" is defined as the mean water-level altitude in meters for the period of analysis relative to NAVD 88. Data for monitoring wells are available in the National Water Information System (U.S. Geological Survey, 2020). CT, Connecticut; RI, Rhode Island]

\begin{tabular}{|c|c|c|c|c|c|c|c|c|}
\hline $\begin{array}{l}\text { Map } \\
\text { no. }\end{array}$ & Site number & Station name & $\begin{array}{l}\text { Decimal } \\
\text { latitude }\end{array}$ & $\begin{array}{l}\text { Decimal } \\
\text { longitude }\end{array}$ & $\begin{array}{l}\text { Land-surface } \\
\text { altitude }\end{array}$ & $\begin{array}{l}\text { Mean water- } \\
\text { level altitude }\end{array}$ & $\begin{array}{c}\text { Number of } \\
\text { measure- } \\
\text { ments }\end{array}$ & Period of analysis \\
\hline 61 & 414237072034401 & CT-SC 20 & 41.7103763 & -72.0617425 & 357.23 & 350.70 & 317 & $\begin{array}{l}12 / 16 / 1983 \text { through } \\
11 / 20 / 2020\end{array}$ \\
\hline 62 & 414240072032201 & CT-SC 21 & 41.71120967 & -72.0556312 & 267.93 & 267.32 & 330 & $\begin{array}{l}12 / 17 / 1983 \text { through } \\
11 / 20 / 2020\end{array}$ \\
\hline 63 & 414240072032202 & CT-SC 23 & 41.71120967 & -72.0556312 & 268.00 & 265.87 & 340 & $\begin{array}{l}12 / 17 / 1983 \text { through } \\
11 / 20 / 2020\end{array}$ \\
\hline 64 & 414240072033201 & CT-SC 22 SCOTLAND & 41.71101667 & -72.0591667 & 327.13 & 315.27 & 334 & $\begin{array}{l}10 / 02 / 1984 \text { through } \\
11 / 20 / 2020\end{array}$ \\
\hline 65 & 414243072040501 & CT-SC 19 & 41.71215278 & -72.0675083 & 494.65 & 489.03 & 343 & $\begin{array}{l}12 / 16 / 1983 \text { through } \\
11 / 20 / 2020\end{array}$ \\
\hline
\end{tabular}




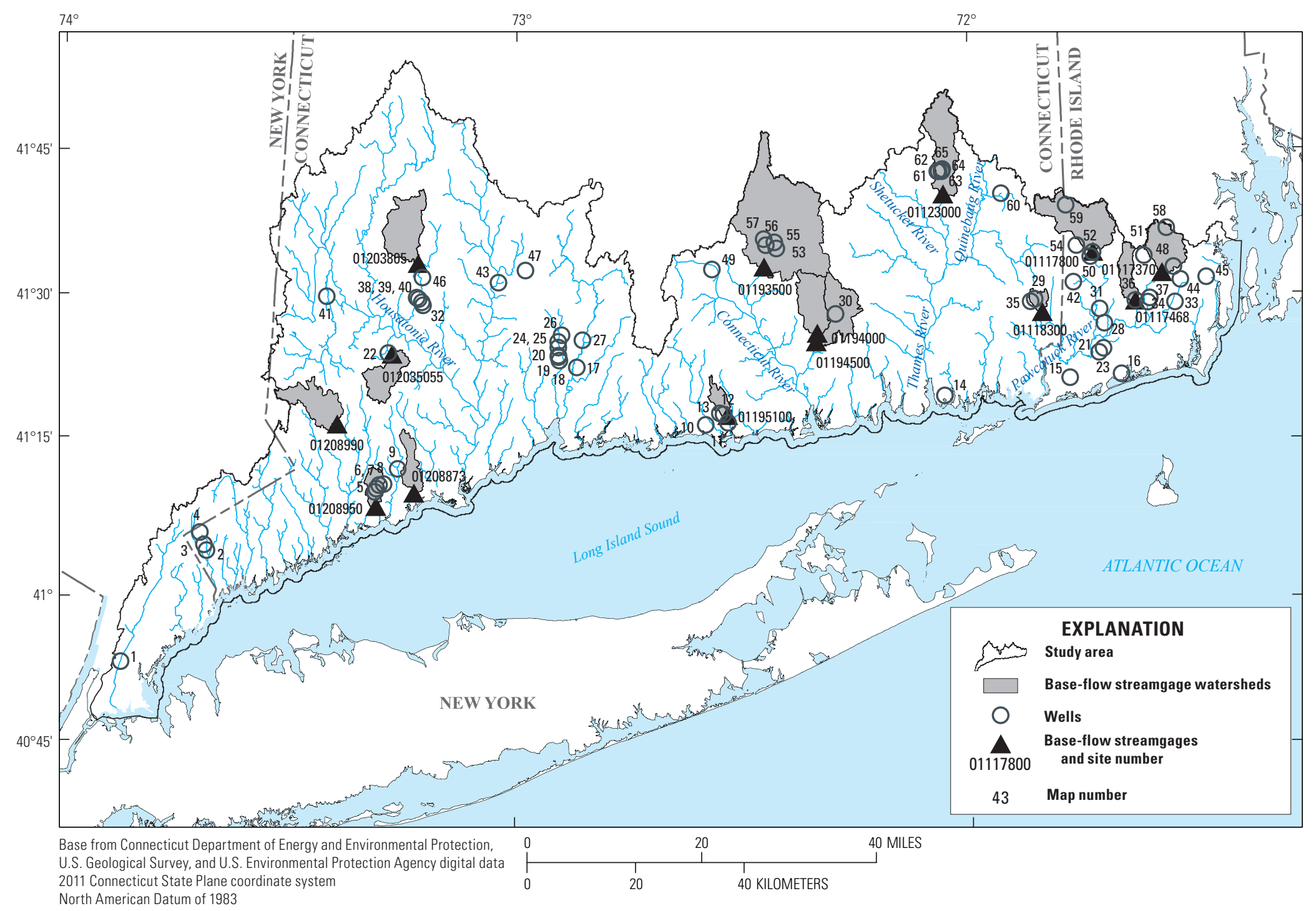

Figure 7. Locations and map numbers of 65 groundwater-monitoring wells and the locations and site numbers of 14 streamgages and their associated watersheds on the north shore of Long Island Sound (coastal Connecticut and adjacent areas of New York and Rhode Island). The wells and streamgages were used for model calibration. The map number of each well is listed in the first column of table 4. The site number of each streamgage is listed in the first column of table 5. Site numbers and associated data are available in the National Water Information System (U.S. Geological Survey, 2020). 
Table 5. U.S. Geological Survey streamgages on the north shore of Long Island Sound (coastal Connecticut and adjacent areas of New York and Rhode Island) that were used for model calibration.

[Drainage area is in square kilometers. Base flow was calculated by using the mean of the PART and BFI algorithms implemented in the DVstats package in R (Lorenz, 2017; R Core Team, 2019). Base flow and total streamflow are given in cubic meters per day. Data for streamgages are available in the National Water Information System (U.S. Geological Survey, 2020). CT, Connecticut; RI, Rhode Island]

\begin{tabular}{|c|c|c|c|c|c|c|c|c|c|}
\hline Site number & Station name & $\begin{array}{l}\text { Decimal } \\
\text { latitude }\end{array}$ & $\begin{array}{l}\text { Decimal } \\
\text { longitude }\end{array}$ & $\begin{array}{l}\text { Drainage } \\
\text { area }\end{array}$ & Period of analysis & $\begin{array}{l}\text { Number } \\
\text { of years }\end{array}$ & $\begin{array}{l}\text { Base- } \\
\text { flow } \\
\text { fraction }\end{array}$ & $\begin{array}{c}\text { Mean } \\
\text { base flow }\end{array}$ & $\begin{array}{c}\text { Total } \\
\text { streamflow }\end{array}$ \\
\hline 01117800 & $\begin{array}{l}\text { WOOD RIVER NEAR } \\
\text { ARCADIA, RI }\end{array}$ & 41.57427256 & -71.72102022 & 91 & $\begin{array}{l}\text { 10/01/1989 through } \\
\text { 09/30/2019 }\end{array}$ & 30 & 0.77 & 142,557 & 184,005 \\
\hline 01194500 & $\begin{array}{l}\text { EAST BRANCH EIGHT- } \\
\text { MILE RIVER NEAR } \\
\text { NORTH LYME, CT }\end{array}$ & 41.42755092 & -72.33470565 & 58 & $\begin{array}{l}\text { 10/01/2002 through } \\
\text { 09/30/2019 }\end{array}$ & 17 & 0.58 & 70,620 & 121,531 \\
\hline 01193500 & $\begin{array}{l}\text { SALMON RIVER NEAR } \\
\text { EAST HAMPTON, CT }\end{array}$ & 41.55234949 & -72.44975686 & 259 & $\begin{array}{l}\text { 10/01/1989 through } \\
09 / 30 / 2019\end{array}$ & 30 & 0.59 & 285,496 & 486,074 \\
\hline 01117370 & $\begin{array}{l}\text { QUEEN R AT LIBERTY RD } \\
\text { AT LIBERTY RI }\end{array}$ & 41.53900091 & -71.56876505 & 51 & $\begin{array}{l}\text { 10/01/1998 through } \\
09 / 30 / 2016\end{array}$ & 18 & 0.72 & 67,365 & 93,938 \\
\hline 01208950 & $\begin{array}{l}\text { SASCO BROOK NEAR } \\
\text { SOUTHPORT, CT }\end{array}$ & 41.15293914 & -73.30598742 & 19 & $\begin{array}{l}\text { 10/01/1989 through } \\
09 / 30 / 2019\end{array}$ & 30 & 0.54 & 18,615 & 34,162 \\
\hline 01117468 & $\begin{array}{l}\text { BEAVER RIVER NEAR } \\
\text { USQUEPAUG, RI }\end{array}$ & 41.49234178 & -71.62817817 & 23 & $\begin{array}{l}\text { 10/01/1989 through } \\
09 / 30 / 2019\end{array}$ & 30 & 0.79 & 39,723 & 50,037 \\
\hline 01208873 & $\begin{array}{l}\text { ROOSTER RIVER AT } \\
\text { FAIRFIELD, CT }\end{array}$ & 41.17571828 & -73.22254791 & 27 & $\begin{array}{l}\text { 10/01/1989 through } \\
09 / 30 / 2017\end{array}$ & 28 & 0.49 & 19,483 & 39,529 \\
\hline 012035055 & $\begin{array}{l}\text { POOTATUCK RIVER AT } \\
\text { BERKSHIRE }\end{array}$ & 41.40656178 & -73.27227848 & 40 & $\begin{array}{l}\text { 10/01/2006 through } \\
\text { 09/30/2019 }\end{array}$ & 13 & 0.61 & 49,998 & 81,462 \\
\hline 01194000 & $\begin{array}{l}\text { EIGHTMILE RIVER AT } \\
\text { NORTH PLAIN, CT }\end{array}$ & 41.44172838 & -72.33254247 & 52 & $\begin{array}{l}\text { 10/01/2008 through } \\
\text { 09/30/2019 }\end{array}$ & 11 & 0.61 & 64,333 & 105,626 \\
\hline 01208990 & $\begin{array}{l}\text { SAUGATUCK RIVER NEAR } \\
\text { REDDING, CT }\end{array}$ & 41.28970875 & -73.39246016 & 54 & $\begin{array}{l}\text { 10/01/1989 through } \\
\text { 09/30/2019 }\end{array}$ & 30 & 0.61 & 61,368 & 100,540 \\
\hline 01195100 & $\begin{array}{l}\text { INDIAN RIVER NEAR } \\
\text { CLINTON, CT }\end{array}$ & 41.30546407 & -72.53090117 & 15 & $\begin{array}{l}\text { 10/01/1989 through } \\
\text { 09/30/2019 }\end{array}$ & 30 & 0.55 & 13,165 & 23,969 \\
\hline 01118300 & $\begin{array}{l}\text { PENDELTON HILL BROOK } \\
\text { NEAR CLARKS FALLS, } \\
\text { CT }\end{array}$ & 41.47487803 & -71.83430835 & 10 & $\begin{array}{l}\text { 10/01/1989 through } \\
\text { 09/30/2019 }\end{array}$ & 30 & 0.62 & 13,672 & 22,214 \\
\hline 01123000 & $\begin{array}{l}\text { LITTLE RIVER NEAR } \\
\text { HANOVER, CT }\end{array}$ & 41.67215691 & -72.05222345 & 78 & $\begin{array}{l}\text { 10/01/1989 through } \\
\text { 09/30/2019 }\end{array}$ & 30 & 0.62 & 85,416 & 137,710 \\
\hline 01203805 & $\begin{array}{l}\text { WEEKEEPEEMEE RIVER } \\
\text { AT HOTCHKISSVILLE, } \\
\text { CT }\end{array}$ & 41.55768198 & -73.21549684 & 69 & $\begin{array}{l}\text { 10/01/2003 through } \\
\text { 09/30/2019 }\end{array}$ & 16 & 0.58 & 75,505 & 130,860 \\
\hline
\end{tabular}




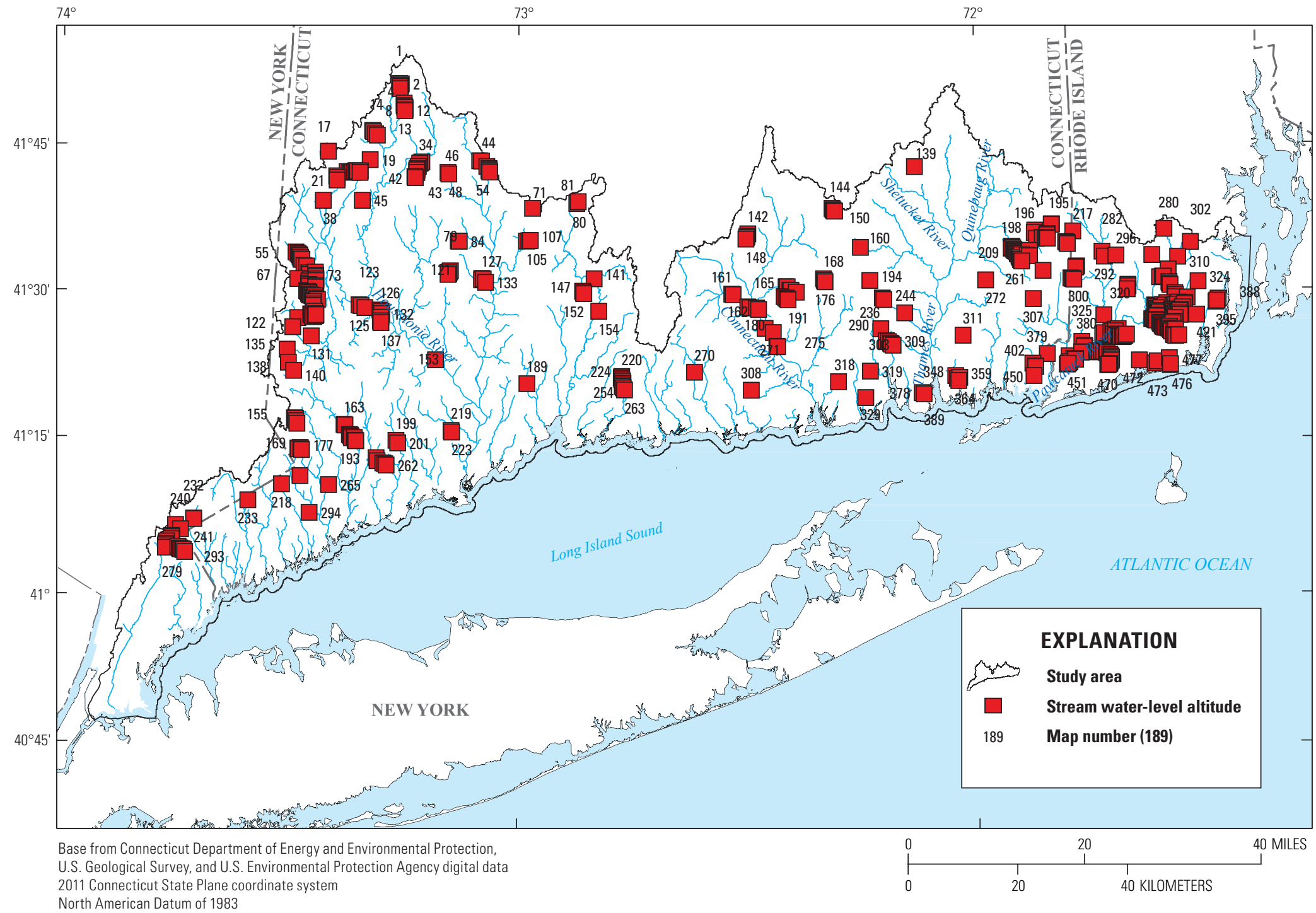

Figure 8. Locations of the calculated stream water-level altitudes on the north shore of Long Island Sound (coastal Connecticut and adjacent areas of New York and Rhode Island) that were used for model calibration. The map number of each stream water-level altitude is listed in Barclay and Mullaney (2021b). 
relative to other withdrawals. Due to the steady-state nature of this analysis and data-availability limitations, constant water-use rates were used. Sources of water-use data are listed in table 6, and withdrawal rates are summarized in table 7 . The approaches used to estimate the water-use data are described below.

Withdrawal locations and rates for private residential wells were estimated on the basis of the extent of public watersupply service areas (fig. 9) (Connecticut Office of Policy and Management, 2019), 2010 population density (Falcone, 2016), and an estimated per capita usage rate of 0.18 cubic meters per day $\left(\mathrm{m}^{3} / \mathrm{d}\right)$ (Dieter and others, 2018). Similarly, septic return-flow locations and rates were estimated on the basis of the extent of sewer-service (CT DEEP, 2019b [updated 1998 data]), population density (Falcone, 2016), an estimated per-capita usage rate of $0.18 \mathrm{~m}^{3} / \mathrm{d}$ (Dieter and others, 2018), and a consumptive-use fraction of 0.15 (Shaffer and Runkle, 2007). This consumptive-use fraction means that in areas with private wells and septic systems, the net loss of water from the groundwater system is 15 percent. In this analysis, it was assumed that private residential wells existed only where public water supply was not available, and that septic

Table 6. Data sources consulted for water-use information.

[All of New York City was assumed to be served by public water supply]

\begin{tabular}{|c|c|c|}
\hline Area & Source & Citation \\
\hline \multicolumn{3}{|c|}{ Public water-supply service areas } \\
\hline New York: New York City & $\begin{array}{l}\text { https://data.cityofnewyork.us/City-Government/Borough- } \\
\text { Boundaries/tqmj-j8zm }\end{array}$ & Department of City Planning, 2019 \\
\hline $\begin{array}{l}\text { New York: Westchester } \\
\text { County }\end{array}$ & $\begin{array}{l}\text { https://gis.westchestergov.com/datasets/wcgis::water- } \\
\text { districts-1/about }\end{array}$ & $\begin{array}{l}\text { Westchester County Department of Planning, } \\
\text { 2015b }\end{array}$ \\
\hline Connecticut & $\begin{array}{l}\text { ftp://ftp.state.ct.us/pub/opm/TCDPP/C\&D\%20 } \\
\text { Plan\%20GIS/GIS/OPM_DRAFT_C\&D_Data_Web/ } \\
\text { WaterService/ }\end{array}$ & $\begin{array}{l}\text { Connecticut Office of Policy and Management, } \\
2019\end{array}$ \\
\hline Rhode Island & https://www.rigis.org/datasets/water-supply-districts & $\begin{array}{l}\text { Rhode Island Geographic Information System, } \\
1995\end{array}$ \\
\hline \multicolumn{3}{|c|}{ Sewer-service areas } \\
\hline New York: New York City & https://openseweratlas.tumblr.com/data & Open Sewer Atlas NYC, 2016 \\
\hline Rhode Island & https://www.rigis.org/datasets/sewered-areas & $\begin{array}{l}\text { Rhode Island Geographic Information System, } \\
2012\end{array}$ \\
\hline \multicolumn{3}{|c|}{ Water-withdrawal rates } \\
\hline New York & $\begin{array}{l}\text { https://gis.ny.gov/gisdata/inventories/details.cfm?DSID= } \\
\quad 1265\end{array}$ & $\begin{array}{l}\text { Division of Water-Bureau of Water Resource } \\
\text { Management, } 2018\end{array}$ \\
\hline Connecticut & $\begin{array}{l}\text { https://pubs.usgs.gov/circ/1405/pdf/circ1405.pdf; htt } \\
\text { ps://www.arcgis.com/home/item.html?id=b5a6750a85cd } \\
\text { 49ffa800e1aab88db95c }\end{array}$ & $\begin{array}{l}\text { Maupin and others, 2014; Levin and others, 2018; } \\
\text { Connecticut Department of Public Health } 2013\end{array}$ \\
\hline Rhode Island & & $\begin{array}{l}\text { Data layer based on Rhode Island Department of } \\
\text { Health data }\end{array}$ \\
\hline
\end{tabular}


Table 7. Withdrawal rates from wells on the north shore of Long Island Sound (coastal Connecticut and adjacent areas of New York and Rhode Island).

[Withdrawal rates are reported in cubic meters per day. Entries in the columns labeled "Minimum," "Median," and "Maximum" are based on the applied pumping rates]

\begin{tabular}{lccccccc}
\hline \multirow{2}{*}{ Type } & \multicolumn{4}{c}{ All wells } & \multicolumn{2}{c}{ Wells with reduced pumping } \\
\cline { 2 - 8 } & $\begin{array}{c}\text { Number of } \\
\text { wells }\end{array}$ & Minimum & Median & Maximum & $\begin{array}{c}\text { Number of } \\
\text { wells }\end{array}$ & $\begin{array}{c}\text { Median } \\
\text { reduction }\end{array}$ & $\begin{array}{c}\text { Percentage } \\
\text { reduction }\end{array}$ \\
\hline Private, residential & 80,415 & 0.54 & 1.03 & 37.74 & 81 & 0.17 & 17.92 \\
Public water supply & 385 & 2.70 & 40.51 & $11,620.00$ & 55 & 492.62 & 54.68 \\
\hline
\end{tabular}

systems existed only where public sewer was not available. No assumptions were made regarding the co-occurrence of public water and public sewer, or private wells and septic systems; private-well withdrawals and septic return flows were estimated separately. Further details of the private-well withdrawals and septic return-flow estimates are given in appendix 2 . The estimated withdrawal rates for private wells are summarized in table 7, and a map of private-well withdrawals, septic return flows, and net groundwater losses due to private-well withdrawals and septic return flows is shown in figure 10.

Water-withdrawal information for public-supply wells was gathered from a variety of sources. Withdrawal information for wells in Rhode Island was previously compiled for the 2015 USGS water-use compilations (Dieter and others, 2018). Withdrawal information for larger wells in Connecticut previously was compiled for the USGS water-use survey in $2010^{1}$ (Maupin and others, 2014) and for the Connecticut Streamflow and Sustainable Water-Use Estimator (Levin and others, 2018). Withdrawal rates for smaller systems in Connecticut were estimated by using the service-area maps, the size of the population served (Connecticut Department of Public Health, 2013; Connecticut Office of Policy and Management, 2019), and an estimated per-capita water-usage rate of $0.18 \mathrm{~m}^{3} / \mathrm{d}$ (Dieter and others, 2018). Withdrawal information for wells in New York came from the New York State Department of Environmental Conservation's Well-Database Program (Division of Water-Bureau of Water Resource Management, 2018).

\footnotetext{
${ }^{1}$ Withdrawal information for Connecticut was based on data for 2010 because data for 2015 was only compiled at the county level and not the well level.
} 


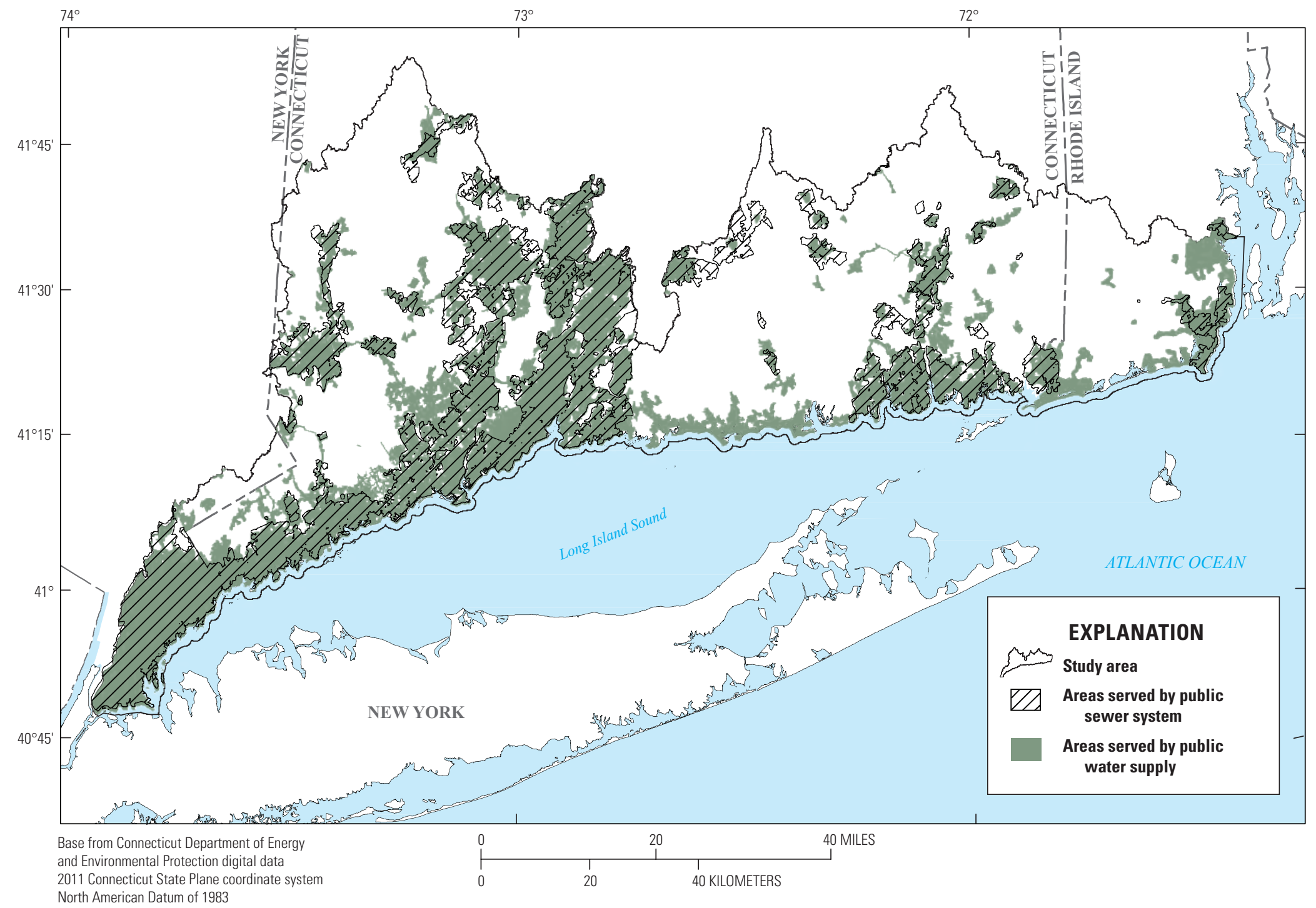

Figure 9. Public water-supply and public sewer service on the north shore of Long Island Sound (coastal Connecticut and adjacent areas of New York and Rhode Island). Sources of the public sewer and public water data are listed in table 6. 


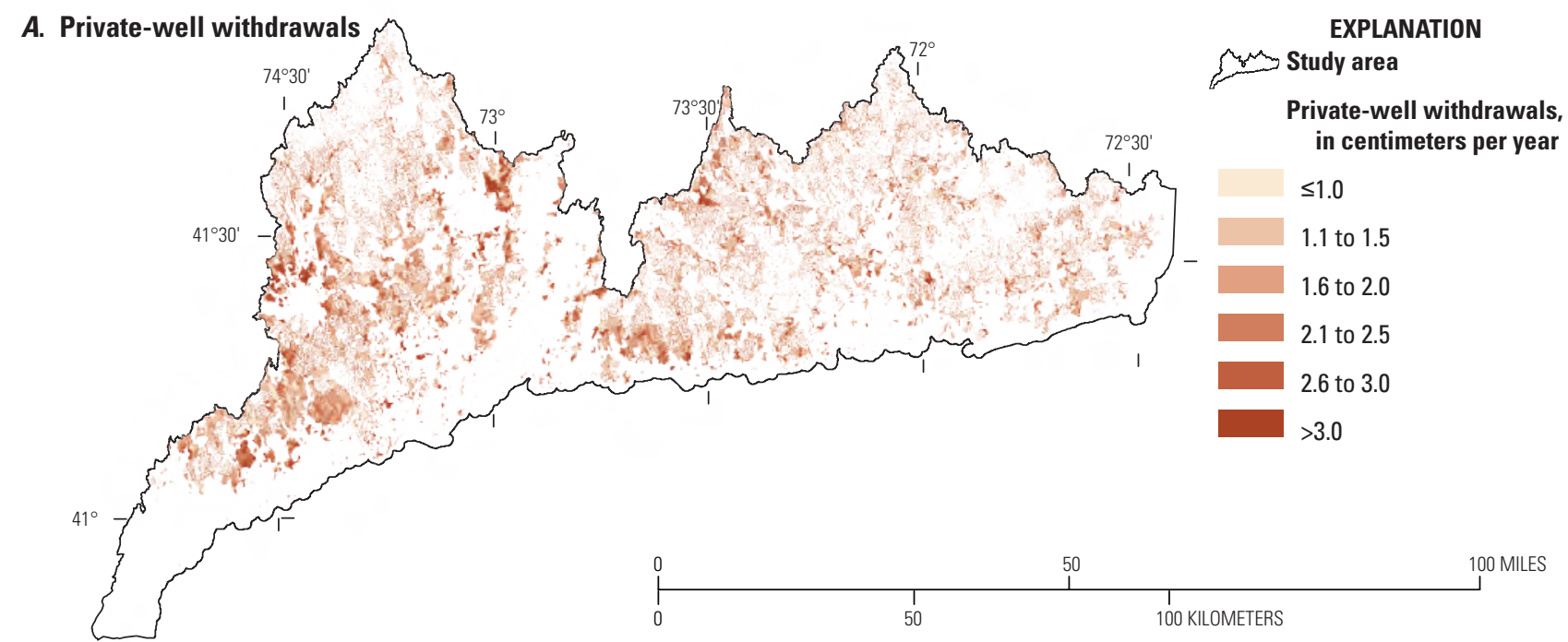

\section{B. Septic return flows}
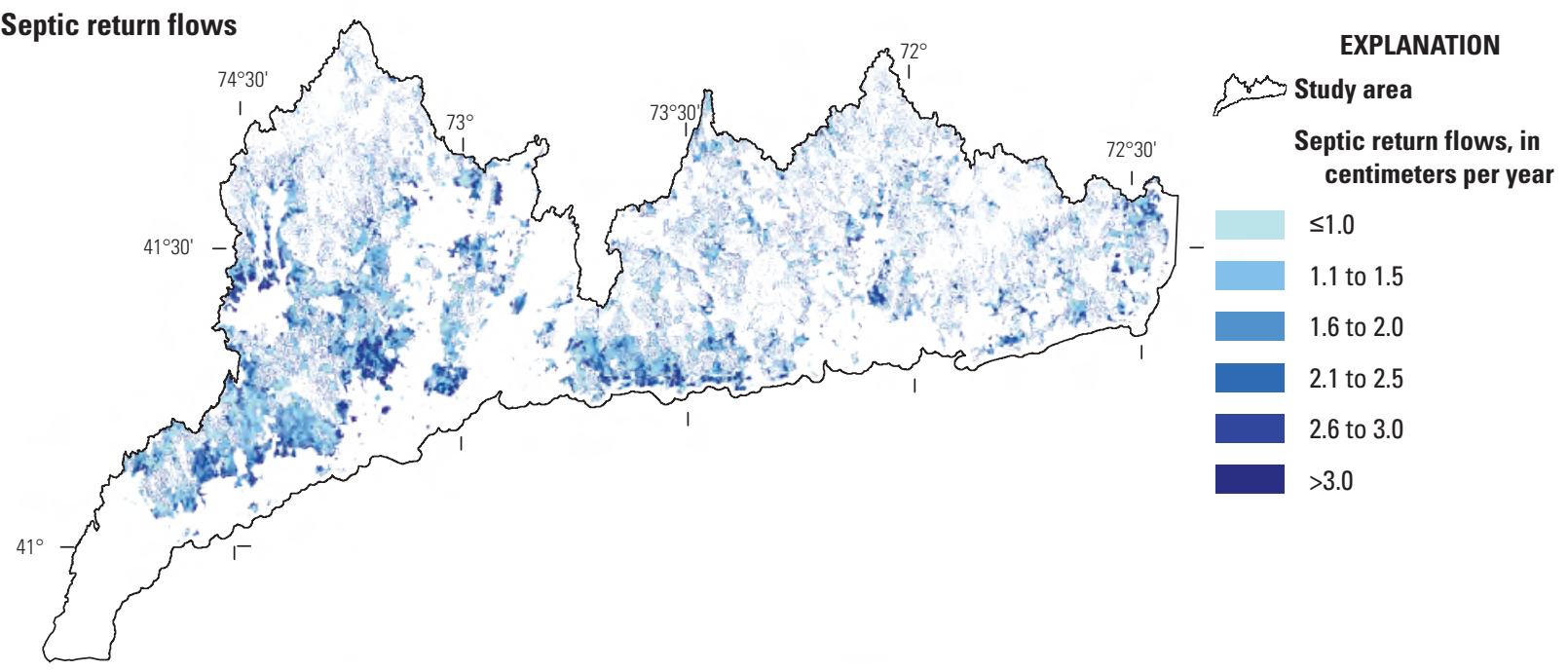

EXPLANATION

C. Net groundwater loss

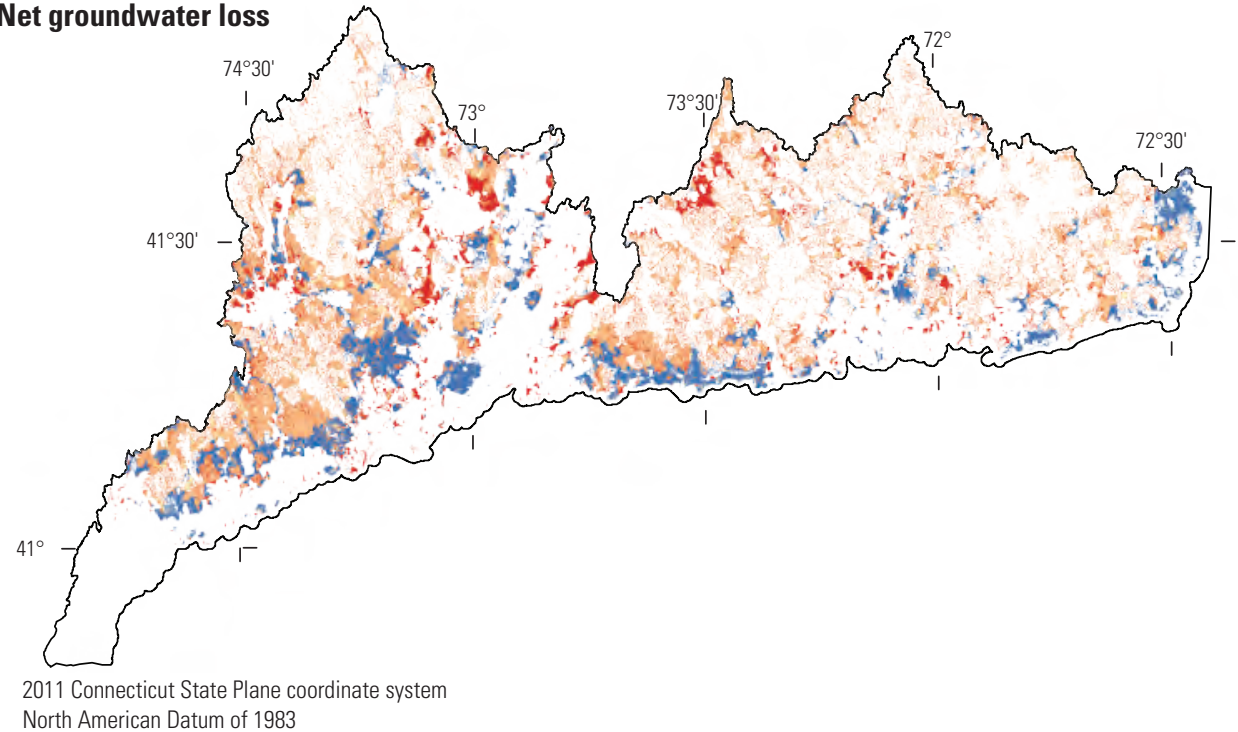

EXPLANATION

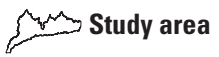

Net groundwater loss, in centimeters per year

$\leq-1.00$

-0.99 to -0.50

-0.49 to -0.15

-0.14 to 0.15

0.16 to 0.50

0.51 to 1.00

$>1.00$

Figure 10. A, estimated private-well withdrawals, $B$, septic return flows, and $C$, net groundwater loss from both private wells and septic return flows on the north shore of Long Island Sound (coastal Connecticut and adjacent areas of New York and Rhode Island). Sources of the service-area data are listed in table 6 , and the estimation method is described in appendix 2. 


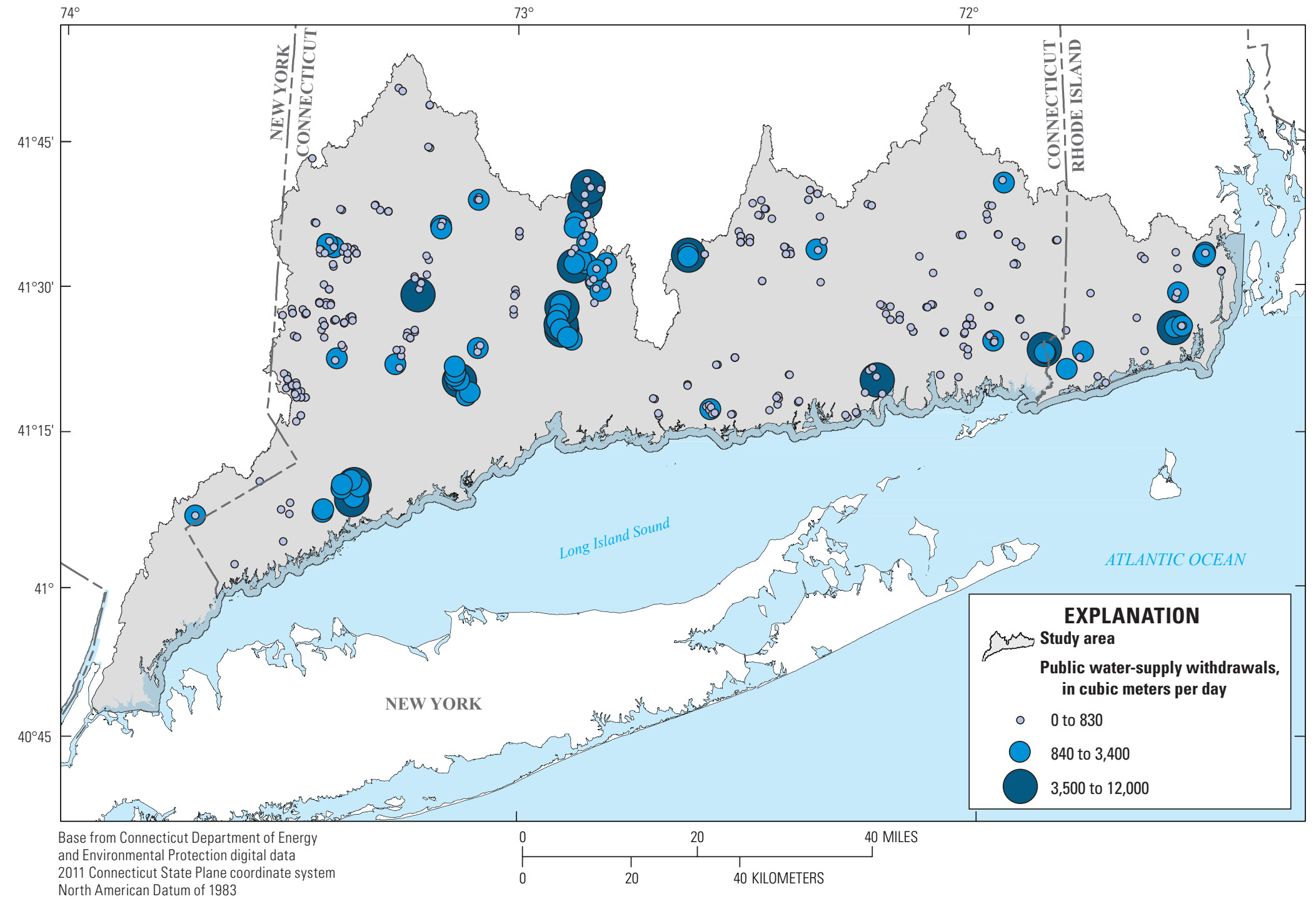

Figure 11. Groundwater withdrawals for public water supply on the north shore of Long Island Sound (coastal Connecticut and adjacent areas of New York and Rhode Island). In Connecticut, only community-public-water-supply wells are included. Sources of public water-supply withdrawal data are listed in table 6. 


\section{Numerical-Model Development}

A steady-state numerical groundwater-flow model of the north shore of the LIS was developed to quantify groundwater fluxes and groundwater travel times to embayments along the northern shore of LIS. Numerical groundwater-flow models, such as the suite of MODFLOW programs (Harbaugh, 2005; Niswonger and others, 2011), represent the subsurface as a three-dimensional grid of cells. The user specifies the properties of each cell, including the hydraulic conductivity, boundary conditions such as water bodies, inflows into the model such as groundwater recharge, and outflows such as groundwater withdrawals. Based on the specified information, the model calculates the groundwater head within each model cell and the flows between the model cells.

The model for this study was developed in MODFLOWNWT (Niswonger and others, 2011) by using a sequence of steps modified from Starn and Carlson (2018) and Starn and Belitz (2018). The approach is described briefly here and in detail in subsequent sections. First, a single-layer, coarse-resolution model was developed. Second, the singlelayer coarse-resolution model was expanded to a four-layer coarse-resolution model. Steps one and two are described in the section "Initial Model Development." Third, the fourlayer model was refined and calibrated to generate the final coarse-resolution model as described in the section "Model Calibration." Fourth, a fine-resolution, four-layer model was developed by using steps one and two and run by using the calibrated values from the coarse-resolution model from step three. This was the final model and is described in the section "Post-Calibration Model Adjustments." The previous four steps involve MODFLOW. The fifth step is setting up MODPATH (Pollock, 2012), a particle-tracking program that uses output from MODFLOW. The MODPATH analysis, which was used to simulate groundwater-flowpaths and estimate groundwater travel times, is described in the section "Particle Tracking Model Development."

Input and output files for the numerical groundwater-flow model and the particle-tracking model are available as a USGS data release (Barclay and Mullaney, 2021a).

\section{Groundwater-Model Design}

The numerical groundwater-flow model was developed by using MODFLOW-NWT (MODFLOW) (Niswonger and others, 2011) and the FloPy package (Bakker and others, 2016) within Python (Oliphant, 2007). Input data were extracted, and model files were compiled, by using variations of the GenMod 1.0 Jupyter notebooks (Starn and Carlson, 2018).

\section{Spatial Extent and Horizontal Model Grid}

The active model domain covers the northern shore of LIS, extending from the East River in New York on the west side to Narragansett Bay on the east side. The southern boundary extends $1 \mathrm{~km}$ into the Sound beyond the coast. The northern boundary extends to HUC12 watershed boundaries; groundwater was assumed not to flow across the northern boundary.

The initial horizontal discretization was $304.8 \mathrm{~m}$ $(1,000 \mathrm{ft})$, with 336 rows and 746 columns. The horizontal discretization was refined after the model calibration to $152.4 \mathrm{~m}$ $(500 \mathrm{ft})$, resulting in 672 rows and 1,491 columns. The final model had 1,001,952 cells in each layer, of which 399,976 were active.

\section{Hydraulic Boundaries}

Surface water, both fresh and saline, is represented by head-dependent flux boundaries; with such boundaries, flow between the surface water and the aquifer is controlled by the level of the surface water body, the groundwater head within the aquifer, and the user-specified sediment properties. Within MODFLOW, head-dependent flux boundaries can be simulated by using several different packages, such as the General-Head Boundary (GHB) and Drain (DRN) (Harbaugh, 2005; Niswonger and Prudic, 2005). These packages differ in the level of complexity with which groundwater-surface water interactions are represented and the degree to which unrealistic outflows from these boundary conditions can be controlled. The GHB package, which is the most general of the head-dependent boundary packages, allows for flow into or out of a model cell based on the model-calculated hydraulic head and user-specified conductance and boundary head. The DRN package is similar to the GHB package, but it uses a different method of calculating flux when the model-calculated head is below the boundary head, in this case the head of the drain. With the DRN package, the flux is zero when the model-calculated hydraulic head is below the user-specified river altitude. Because the DRN package does not allow flow from surface water into groundwater, it prevents unrealistically large inflows of water to the groundwater system from the surface-water boundary that can occur with the GHB package, but in doing so, it does not account for the contribution of surface water from losing stream reaches to the groundwater system. Other packages, such as the SFR2 package, may better represent simulated fluxes on the basis of upstream exchange and stream-channel geometry; however, the user-specified inputs to SFR2 are considerably more involved than for the DRN package and thus are beyond the scope of this study.

\section{Simulation of Freshwater Boundaries}

Freshwater streams and rivers were simulated by use of the DRN package. Stream water-level altitudes were extracted from the NHDPlus stream-altitude dataset (U.S. 
Environmental Protection Agency and U.S. Geological Survey, 2012) and linearly interpolated to the model grid. Minor corrections were made to stream water-level-altitude estimates to ensure that stream water-level altitudes decreased monotonically or were level in the downstream direction. Stream water-level altitudes below $0 \mathrm{~m}$ relative to NAVD 88 were set to $0 \mathrm{~m}$ to ensure smooth boundaries with sea level. The boundary conductance was calculated as the product of the streambed hydraulic conductivity, the segment length, the stream width, and the streambed thickness. Based on the preliminary calibration analysis completed as part of this study, the streambed hydraulic-conductivity values were set to 0.91 meter per day $(\mathrm{m} / \mathrm{d})$ for fine sediments and $9.1 \mathrm{~m} / \mathrm{d}$ for coarse sediments; these values were not adjusted during the calibration. The segment length was extracted from the NHDPlus dataset. The stream width was estimated by the use of a geomorphic scaling relation based on the sum of upstream river lengths as described in appendix 3. A value of $0.305 \mathrm{~m}$ was used for the streambed thickness for all streams.

Lakes within the study area were assumed to be well connected to the underlying aquifer and therefore to serve as preferential flowpaths where flow is essentially unimpeded (Masterson and Granato, 2013; Walter and others, 2019). For this reason, lakes were simulated as areas of high hydraulic conductivity. Model cells with their horizontal centroid within a waterbody as mapped in the NHDPlus, version 2.1, dataset (U.S. Environmental Protection Agency and U.S. Geological Survey, 2012) were classified as lakes, and the horizontal hydraulic conductivity in the uppermost model layer was set to $3,048 \mathrm{~m} / \mathrm{d}$, a value more than 200 times greater than the highest hydraulic conductivity for terrestrial sediments, such that water flow is relatively unimpeded. This value was not adjusted during the calibration process.

In the northeastern United States, most surface-water bodies are hydrologically connected to a stream network. Because of discretization issues in both the model grid and the digitized stream network, however, small streams and waterbodies that are, in reality, hydrologically connected to the stream network, are disconnected from the stream network in the model grid. This is particularly an issue in areas of steeper topography and thinner surficial sediments, where the bottom of the surficial layers in one model cell may be substantially higher than the top of the surficial layers in the adjacent cell.

To correct for erroneous hydrologically disconnected waterbodies, additional head-dependent flux boundaries were added to all waterbody cells by use of the DRN package. The percentage of each cell covered by waterbodies, as mapped by the National Wetland Inventory (U.S. Fish and Wildlife Service, 2018), was calculated. Drains were added to all active cells with waterbodies that did not already have a stream drain, and the conductance was adjusted for cells with existing stream drains. In cells with waterbodies and stream drains, the streambed area was adjusted to equal the larger value of the previously calculated streambed area and the area of the cell covered by water. For new drains, the altitude of the drain was set at the land surface, and the conductance of the drain was calculated as the product of the cell area covered by water, the bed-sediment thickness, and the bed-sediment hydraulic conductivity. As for the stream drains, the bed-sediment hydraulic conductivity was set to $0.91 \mathrm{~m} / \mathrm{d}$ for fine sediments and $9.1 \mathrm{~m} / \mathrm{d}$ for coarse sediments on the basis of the preliminary calibration analysis in this study; the sediment thickness was assumed to be $0.305 \mathrm{~m}$.

\section{Simulation of the Marine Boundary}

The overall spatial extent of the marine boundary was delineated by selecting areas classified as "Estuarine and Marine Deepwater" or "Estuarine and Marine Wetlands" in the National Wetlands Inventory (U.S. Fish and Wildlife Service, 2018). Areas of water within the marine boundary were collectively named "coastal waters"; all other areas were named "inland waters." The marine boundary was simulated by using a DRN for cells within the base HUC12 watershed boundaries (fig. 12) and a GHB for cells that extend beyond the HUC12 boundaries into LIS. The DRN package was used for the cells within the base HUC12 boundaries to prevent unrealistically large flows of water from surface water into the aquifer. Based on preliminary analysis in this study, these inflows from surface water to groundwater were less common in the open coastal waters beyond the HUC12 boundaries; for this reason, the GHB package, which better represents flow between coastal waters and groundwater, was used in cells beyond the HUC12 boundaries. For both the DRN and GHB packages, the altitude of the marine boundary (water-table altitude) was set according to the following rules to ensure that the boundaries sloped downward toward sea level in LIS: (1) the altitude of the marine boundary for all cells on rivers was set to the correct river-water-level altitude, with all altitudes set to $0 \mathrm{~m}$ or greater; and (2) for cells seaward of the HUC12 boundaries, the boundary altitude was set to $0 \mathrm{~m}$, and the water was assumed to be saline. The saltwater head was converted to an equivalent freshwater head by the use of a saltwater density of 1,022 kilograms per cubic meter $\left(\mathrm{kg} / \mathrm{m}^{3}\right)$ and a freshwater density of $1,000 \mathrm{~kg} / \mathrm{m}^{3}$ in the following equation (Befus and others, 2017):

$$
h_{f}=\left(\frac{\rho_{s}}{\rho_{f}}\right) h_{s}-\left(\frac{\rho_{s}-\rho_{f}}{\rho_{f}}\right) z_{s},
$$

where

$h_{f} \quad$ is the equivalent freshwater head, in m;

$\rho_{s} \quad$ is the density of saltwater, in $\mathrm{kg} / \mathrm{m}^{3}$;

$\rho_{f} \quad$ is the density of freshwater, in $\mathrm{kg} / \mathrm{m}^{3}$;

$h_{s} \quad$ is the saltwater head (sea level), in m; and

$z_{s} \quad$ is the altitude of the sea floor, in $\mathrm{m}$ below the saltwater head (sea-level).

The conductance was calculated as the product of the sediment thickness, the boundary-cell area, and the vertical hydraulic conductivity of the sediment. Marine-sediment thickness was assumed to be uniform at $5 \mathrm{~m}$ (Befus and others, 
2017; Thompson and others, 2007), the hydraulic conductivity was set at $0.61 \mathrm{~m} / \mathrm{d}$, and the area was defined as the fraction of the cell area covered by marine or estuarine wetlands.

\section{Aquifer Properties}

The thickness of unconsolidated surficial materials was derived from a national dataset of aquifer characteristics for the glaciated United States (Yager and others, 2018a). Data from a digital map of glacial sediment thickness for Connecticut (Long Island Sound Resource Center and U.S. Geological Survey, 2004) was used to fill in areas along the marine boundary that were not included in the national aquifer-characteristics file. The national dataset provided more precise estimates of sediment thickness than the Connecticut dataset, which contained thickness ranges rather than single estimates. The Connecticut dataset, however, better represented localized areas of thick sediment than the national dataset. For this reason, the mean of the estimated ranges from the Connecticut dataset was used whenever that estimate was larger than the estimate from the national dataset, unless the range from the Connecticut dataset was the thinnest range $(0-15.2 \mathrm{~m})$, in which case the national value was used. For a small number of model cells that were mapped to the thinnest range in the Connecticut dataset and missing from the national dataset, the thickness was interpolated from surrounding cells by using a grid interpolation first and then a nearest-neighbor algorithm. The grid interpolation was used for 1,845 cells, or 1.8 percent of active cells, and the nearest-neighbor algorithm was used for 38 cells, or 0.04 percent of active cells.

Initial hydraulic-conductivity values were estimated by using simple classifications in which sediments were identified as coarse or fine on the basis of the presence or absence of coarse stratified sediments as mapped in the hydrogeologic framework for surficial aquifers in the glaciated United States (Haj and others, 2018). The initial horizontal hydraulic conductivity was set to $0.91 \mathrm{~m} / \mathrm{d}$ in areas of fine sediments and to $9.1 \mathrm{~m} / \mathrm{d}$ in areas of coarse sediments on the basis of preliminary calibration runs conducted as part of this study. Bedrock formations were not classified by rock type in either the single-layer model or the four-layer model. The initial horizontal hydraulic conductivity in the bedrock was set to $0.3048 \mathrm{~m} / \mathrm{d}$ based on preliminary calibration runs conducted as part of this study. Vertical and horizontal anisotropy values were set to 1 for all cells. During the calibration process, additional surficial sediment and bedrock types were used to better represent aquifer conditions - in particular, hydraulic conductivity and vertical anisotropy. These sediment and bedrock types were identified by the use of geologic maps (table 3) and are further described in the subsequent section "Hydraulic Conductivity Parameters."

\section{Hydraulic Stresses}

Initial groundwater-recharge rates from precipitation were based on recharge rates from a soil water-balance (SWB) model developed for the glaciated United States (Yager and others, 2018a), which estimates groundwater recharge by using a Thornthwaite-Mather water-balance estimate based on precipitation, land cover, soil, and topographic data (Westenbroek and others, 2010). The groundwaterrecharge rates were adjusted as the first step in the model calibration.

Septic return flows were simulated as additional recharge. Areas with septic return flows were identified as areas without public sewer service. As described in appendix 2, the rates for areas with septic return flows were estimated by using the population density, the average waterusage rate per resident, and a consumptive fraction. In most areas, septic return flows represent the internal cycling of water from withdrawal from a private well to on-site return minus a consumptive fraction. In some areas, however, septic systems are coupled with public water supply, in which case the septic return flow may represent a net import of water from outside the local area (fig. 10C). In areas with septic return flows, the rates ranged from less than $0.1 \mathrm{~cm} / \mathrm{yr}$ to $96 \mathrm{~cm} / \mathrm{yr}$, with a median of $1.35 \mathrm{~cm} / \mathrm{yr}$ (fig. 10B).

Water-supply-well withdrawal rates and locations were compiled and estimated from a variety of State-level datasets (table 6, figs. 10A and 11). Estimates of the vertical locations of withdrawals from public water-supply wells were based on available data, which differed by State. Simulated withdrawals of water from public water-supply wells in Connecticut with associated aquifer-protection areas were from the deepest surficial material layer (model layer 3 for most wells); withdrawals from all other public water-supply wells in Connecticut were from the lowest layer (model layer 4), which is a bedrock layer. The Rhode Island dataset of public water-supply wells and their environments included the geologic material; withdrawals from wells with a material code of "SD" or "SD/R" were taken from the deepest surficial-material layer (model layer 3); withdrawals for all other wells (coded "B" or no data) were taken from the lowest layer. The two wells in New York did not have geologic material data; these wells were assumed to be bedrock wells, and their associated withdrawals were from model layer 4, the deepest layer. Public water-supply withdrawal rates were aggregated to the model-cell resolution and ranged from 2.7 to $11,620 \mathrm{~m}^{3} / \mathrm{d}$ (710 gal/d to 3.1 million gallons per day [Mgal/d] $)$ with a median of $40.51 \mathrm{~m}^{3} / \mathrm{d}(11,000 \mathrm{gal} / \mathrm{d})$ (table 7).

Areas without public water supply were assumed to be served by private residential wells. The rates were estimated on the basis of population density and per-resident water usage. Because these wells are typically finished in bedrock, they were assumed to be finished in model layer 4 . Residential-well withdrawals were calculated for each model 


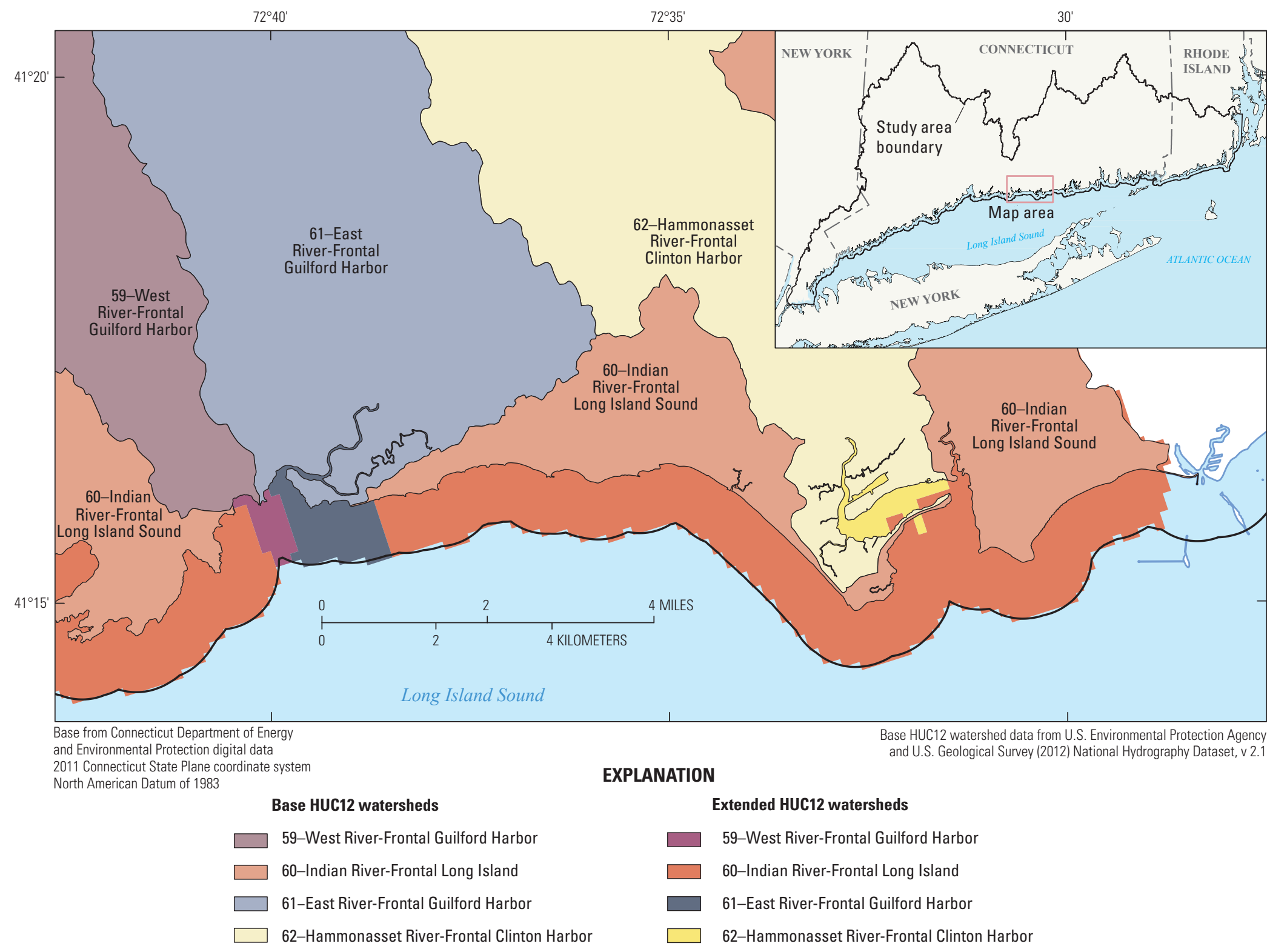

Figure 12. Base and extended HUC12 boundaries in a subset of the study area in coastal Connecticut. The map number of each watershed is listed in the first column of table 1. 
cell with private wells and ranged from 0.54 to $37.74 \mathrm{~m}^{3} / \mathrm{d}$ (140 to $10,000 \mathrm{gal} / \mathrm{d})$ with a median of $1.03 \mathrm{~m}^{3} / \mathrm{d}(270 \mathrm{gal} / \mathrm{d})$ (table 7, fig. 10A). The wells were simulated by the use of the WEL package in MODFLOW (Harbaugh, 2005).

\section{Initial Model Development}

First, a single-layer model was developed that simulated flows through unconsolidated surficial materials. The top of the model was set to the altitude of the land surface, and the bottom of the model was set to the altitude of the top of the bedrock as calculated by subtracting the thickness of the surficial sediments from the altitude of the land surface. In model cells where the thickness of the top of the bedrock was less than $4.57 \mathrm{~m}$, the thickness was increased to equal $4.57 \mathrm{~m}$ to facilitate model convergence. If the top of the bedrock was above the vertical center of a cell, then the cell was classified as bedrock; otherwise, it was classified as the overlying surficial material. The purpose of this model was to estimate the water-table altitude that would be used as the altitude of the top of the four-layer model.

Next, the single-layer model was expanded into a fourlayer model that simulated flows through both unconsolidated surficial materials and bedrock. The top of the four-layer model was derived from the simulated water table in the single-layer model. The simulated water table was modified in the following ways to calculate the altitude of the upper boundary of the four-layer model.

1. In cells that were dry, or where the actual pumping rates had been reduced to prevent the cell from going dry, the land surface, instead of the simulated water table, was used as the upper boundary.

2. The altitude of the upper boundary was set to 120 percent of the absolute value of the simulated water table as a buffer to prevent underestimation of the altitude of the upper boundary.

3. The final upper boundary was adjusted to be at or below the land surface and at or above the water-level altitude of all streams and rivers.

The combined thickness of model layers 1 through 3 was calculated as the difference between the altitudes of the new upper boundary and the top of the bedrock. In areas where the resulting thickness was less than $4.57 \mathrm{~m}$, the thickness was increased to equal $4.57 \mathrm{~m}$ to facilitate model convergence. The calculated thickness was then evenly divided among model layers 1 through 3 . The thickness of the layer that represented bedrock (layer 4), was set to $30.48 \mathrm{~m}$.

To classify each cell as bedrock or surficial material, the altitude of the bedrock surface was intersected with the three-dimensional model grid. If the altitude of the top of the bedrock was higher than the vertical center of the cell, the cell was classified as bedrock; otherwise, it was classified as unconsolidated surficial material. Unconsolidated surficial material covers 90 percent of layer 1 (the uppermost layer), 78 percent of layer 2, and 56 percent of layer 3. Layer 4 represents bedrock throughout the study area.

\section{Model Calibration}

The model was calibrated in two phases. First, the groundwater-recharge estimates were adjusted by use of a linear regression, recharge estimates from the SWB model, and calculated base flow from 14 streamgages. ${ }^{2}$ Hydraulicconductivity parameters were then calibrated by using PEST ++ calibration software (Welter and others, 2015). With PEST ++ , the user identifies a set of hydrologic observations such as water levels in wells and streamflows at streamgages. PEST ++ attempts to reduce the difference between observed and simulated values by systematically adjusting the values of model parameters within user-specified boundaries. This difference, termed the objective function, is calculated as the sum of the squared weighted residuals for each observation. The residual is calculated by subtracting the simulated value from the observed value, with positive residuals indicating that the value was undersimulated and negative values indicating that the value was over-simulated. The goal of the calibration process is to minimize the value of the objective function.

\section{Groundwater-Recharge Calibration}

Groundwater-recharge rates were modified from those estimated with a national SWB model (Yager and others, 2018a) to better match conditions within the study area. Rates were modified by using scaling factors that were estimated by regressing base-flow values on the SWB estimated recharge to coarse and fine sediments, with an adjustment for pervious cover. Base flow from 14 streamgages that were relatively unaffected by water withdrawals were used for this analysis (fig. 7, table 5). Two algorithms were used to calculate base flow-PART (Rutledge, 1998) and BFI (Gustard and others, 1992) — and the mean of the two values, normalized by the watershed area, was used in the regression for each streamflow gage. For this analysis, general groupings of coarse and fine sediments were used. Coarse sediments included "Stratified Glacial Deposits-High Conductivity" and "Stratified Glacial Deposits-Intermediate Conductivity" (table 8). Fine sediments included all other sediment types. A pervious-cover scalar was added to better simulate recharge in urban areas, where impervious cover reduces infiltration and groundwater recharge. Within each base-flow-streamgage watershed, the total recharge to each general sediment group was normalized by the total watershed area.

\footnotetext{
${ }^{2}$ Details of the recharge calibration are in the subsequent section, "Groundwater-Recharge Calibration."
} 
Table 8. Groundwater-recharge rates by sediment type on the north shore of Long Island Sound (coastal Connecticut and adjacent areas of New York and Rhode Island).

[Recharge rates are reported in centimeters per year; Soil Water Balance (SWB) estimates are from Yager and others, 2018a]

\begin{tabular}{lcccccc}
\hline \multirow{2}{*}{ Sediment type } & \multicolumn{3}{c}{ SWB estimates } & \multicolumn{3}{c}{ After calibration } \\
\cline { 2 - 7 } & $\begin{array}{c}\text { 25th } \\
\text { percentile }\end{array}$ & Median & $\begin{array}{c}\text { 75th } \\
\text { percentile }\end{array}$ & $\begin{array}{c}\text { 25th } \\
\text { percentile }\end{array}$ & Median & $\begin{array}{c}\text { 75th } \\
\text { percentile }\end{array}$ \\
\hline Entire study area & 45.17 & 49.97 & 54.98 & 25.72 & 30.11 & 34.27 \\
Alluvium & 47.87 & 54.33 & 60.25 & 26.14 & 30.81 & 34.76 \\
Artificial fill & 0.00 & 38.81 & 61.37 & 0.00 & 7.87 & 21.84 \\
Stratified glacial deposits_-high conductivity & 46.93 & 54.59 & 60.79 & 46.69 & 66.92 & 77.82 \\
Stratified glacial deposits—-intermediate & 53.97 & 60.15 & 65.34 & 31.85 & 53.89 & 71.49 \\
$\quad$ conductivity & & & & & & \\
Stratified glacial deposits—low conductivity & 49.57 & 58.12 & 65.40 & 22.00 & 27.66 & 32.61 \\
Marsh sediments & 45.37 & 51.35 & 56.55 & 26.75 & 31.10 & 34.30 \\
Thick glacial till & 45.08 & 48.74 & 52.45 & 24.53 & 28.27 & 31.06 \\
Glacial till & 44.99 & 49.29 & 53.43 & 25.44 & 29.38 & 32.17 \\
Exposed bedrock & 20.15 & 39.57 & 47.93 & 6.66 & 22.00 & 29.16 \\
\hline
\end{tabular}

The base flow and recharge were fit to a linear-regression equation of the following form:

$$
q=c^{*} F_{\text {pervious }}\left(a^{*} R_{\text {coarse }}+b^{*} R_{\text {fine }}\right),
$$

where

$$
\begin{array}{cc}
q & \begin{array}{c}
\text { is the calculated base flow, in meters per year; } \\
R_{\text {coarse }} \\
\text { is the total SWB estimated recharge to coarse } \\
\text { sediments, in meters per year; }
\end{array} \\
R_{\text {fine }} & \begin{array}{c}
\text { is the total SWB estimated recharge to fine } \\
\text { sediments, in meters per year; }
\end{array} \\
F_{\text {pervious }} & \begin{array}{c}
\text { is the fraction of the watershed with pervious } \\
\text { cover; and }
\end{array} \\
a, b \text {, and } c \quad \text { are all regression coefficients. }
\end{array}
$$

The linear-equation coefficients were calculated to be $a=1.39, b=0.62$, and $c=1$. The coefficient of determination $\left(R^{2}\right.$ value) for the regression equation was 0.99 (fig. 13). These coefficients were applied to the SWB estimated-recharge rates in the model and were not further adjusted during the model calibration. This approach allowed the total volume of groundwater recharge and the distribution of groundwater recharge between coarse and fine sediments to vary, but also preserved the spatial distribution of groundwater recharge simulated by the SWB model (Yager and others, 2018a).

Across the entire study area, the median of the adjusted recharge rate from natural precipitation was $30.11 \mathrm{~cm} / \mathrm{yr}$, and median rates by sediment type ranged from $7.87 \mathrm{~cm} / \mathrm{yr}$ for artificial fill to $66.92 \mathrm{~cm} / \mathrm{yr}$ for stratified glacial deposits of high conductivity (table 8 ) and tended to be higher in the eastern parts of the study area (fig. 14). The adjusted values were lower than those estimated by SWB. The median rate from SWB was $50.0 \mathrm{~cm} / \mathrm{yr}$, and median rates by sediment type ranged from $38.8 \mathrm{~cm} / \mathrm{yr}$ for artificial fill to $60.2 \mathrm{~cm} / \mathrm{yr}$ for stratified glacial deposits (intermediate conductivity).

\section{Hydraulic-Conductivity Parameters}

Horizontal hydraulic-conductivity values throughout the study area were represented by uniform horizontal hydraulicconductivity values in each of 12 geology-based zones (table 9). Geologic zones representing surficial sediments and bedrock were derived from geology maps of Connecticut, New York, and Rhode Island (table 3). Because glacial-till sediments are typically relatively thin, with water levels frequently dropping into the bedrock, in this model the thin glacial till and the underlying bedrock were represented as a combined "glacial till-bedrock complex" for the purpose of calibrating the hydraulic conductivity. Initial values were based on previous groundwater-modeling work in the region (Lyford and others, 2007; Masterson and Granato, 2013; Melvin and others, 1992; Starn and Brown, 2007) and literature values (Domenico and Schwartz, 1997). Vertical-anisotropy values were also updated from the default value of 1 used in the initial model to values based on the 12 geologic zones (table 10) and literature values (Lyford and others, 2007; Masterson and Granato, 2013). These values were not adjusted during the calibration because of the lack of available data.

\section{Observations}

Four primary types of observations-water levels in monitoring wells, stream water-level altitudes, streamflows under base-flow conditions, and the general position of the 


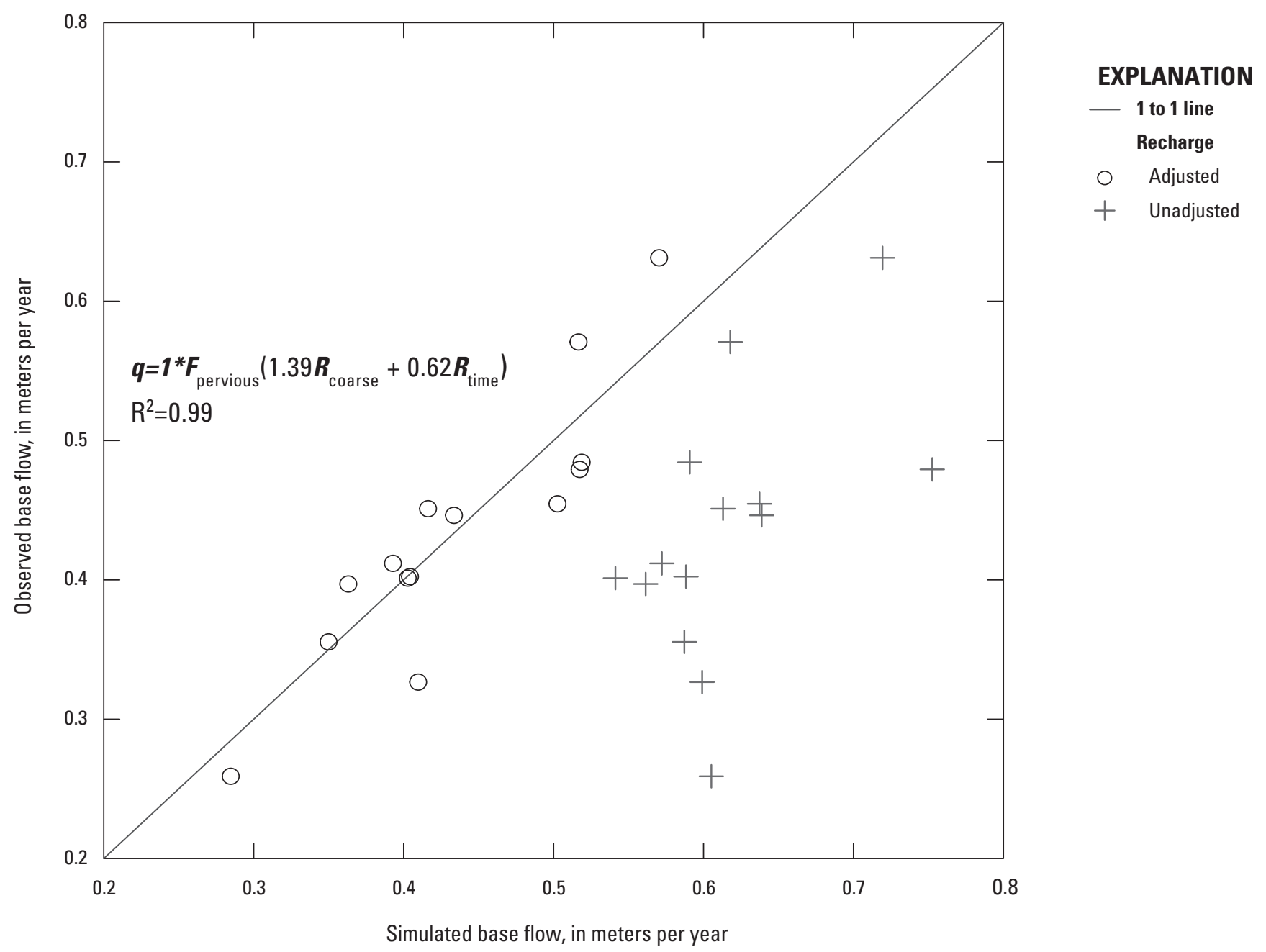

Figure 13. Observed and simulated base-flow values for 14 streamgages on the north shore of Long Island Sound (coastal Connecticut and adjacent areas of New York and Rhode Island). Observed base flow is the mean annual base flow calculated by the PART and BFI algorithms, whereas simulated base flow consists of the initial recharge values adjusted or unadjusted by the regression coefficients. Initial recharge values are from the Soil Water Balance (SWB) model (Yager and others, 2018a). $F_{\text {pervious }}$ fraction of the watershed with pervious cover; $q$, base flow in meters per year $(\mathrm{m} / \mathrm{yr}) ; R_{\text {coarse }}$, SWB recharge to coarse sediments in $\mathrm{m} / \mathrm{yr} ; R_{\text {fine, }}$ SWB recharge to fine sediments in $\mathrm{m} / \mathrm{yr} ; R^{2}$, coefficient of determination.

water table (as indicated by flooded land and dry streams) were used to calibrate the model. The weights of the individual observations within each group are given in table 11 . The weights were estimated on the basis of initial calibration analyses so that the final objective function for each observation group would be of a similar magnitude.

\section{Groundwater Levels}

Measured water levels in monitoring wells describe the water table at specific locations and times. In this study, mean water levels in 65 long-term monitoring wells during water years $^{3} 1999$ through 2018 were used to calibrate the model

\footnotetext{
${ }^{3}$ The water year is defined as the 12-month period from October 1 through September 30 of the next year and designated by the calendar year in which it ends.
}

(fig. 7, table 4) These wells were selected on the basis of the number of measurements (50 or more measurements). The period of analysis for most wells was water year 1999 through water year 2018. Eight wells had shorter periods of analysis, ranging from 8.7 to 17 years, with a median of 15 years. Wells located in artificial fill and those without recorded depths were not used. Individual water-level measurements with measurement accuracies of $0.3048 \mathrm{~m}$ or greater also were excluded. All water-level measurements were converted to their values with respect to the NAVD 88 datum prior to analysis. For wells located in unconsolidated sediments, the simulated water table at the location of the well was compared with the observed mean water-level altitude in the well. For wells located in bedrock, the altitude of the well bottom relative to the altitude of the center of the 30.48-m-thick bedrock layer was used to vertically interpolate the simulated water 


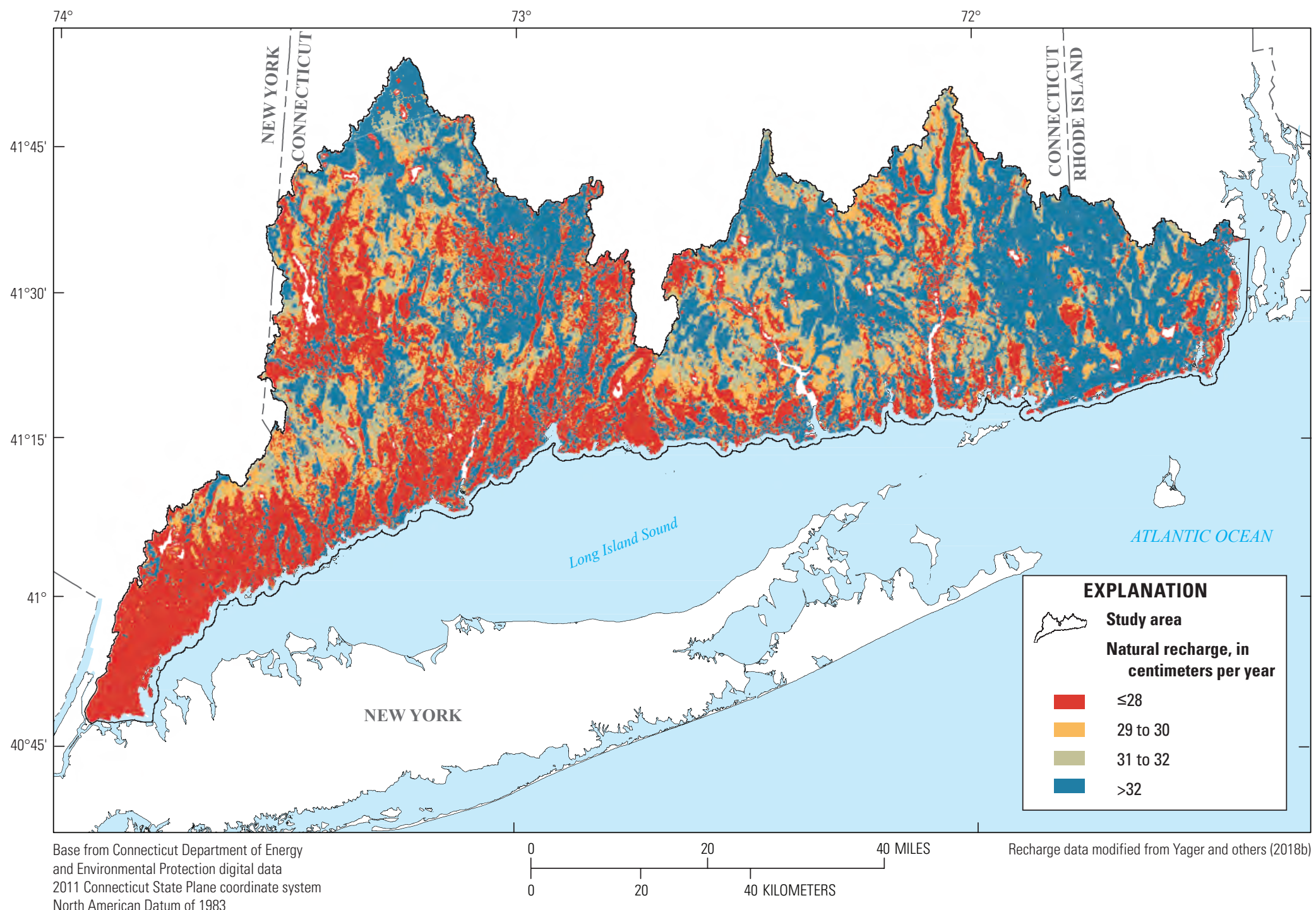

Figure 14. Estimated natural groundwater-recharge rates on the north shore of Long Island Sound (coastal Connecticut and adjacent areas of New York and Rhode Island). These rates do not include septic return flows. 
Table 9. Model-calibration parameters.

$[\mathrm{m} / \mathrm{d}$, meter per day]

\begin{tabular}{|c|c|c|c|c|}
\hline Parameter & Units & Initial value & Final value & Calibration range \\
\hline \multicolumn{5}{|c|}{ Groundwater-recharge multiplier } \\
\hline rch_fine & Dimensionless & 1 & 0.62 & Fixed \\
\hline rch_imp & Dimensionless & 0 & 1 & Fixed \\
\hline \multicolumn{5}{|c|}{ Horizontal hydraulic conductivity } \\
\hline Stratified glacial deposits — high conductivity & $\mathrm{m} / \mathrm{d}$ & 6 & 11 & $1.5-150$ \\
\hline Stratified glacial deposits-intermediate conductivity & $\mathrm{m} / \mathrm{d}$ & 3 & 5.8 & $1.5-150$ \\
\hline Stratified glacial deposits - low conductivity & $\mathrm{m} / \mathrm{d}$ & 3 & 2.6 & $0.003-50$ \\
\hline Marsh sediments & $\mathrm{m} / \mathrm{d}$ & 0.03 & 0.15 & $0.0003-3.0$ \\
\hline Metamorphic bedrock & $\mathrm{m} / \mathrm{d}$ & 0.3 & 0.13 & $0.003-1.0$ \\
\hline Metamorphic/sedimentary bedrock & $\mathrm{m} / \mathrm{d}$ & 0.06 & 0.0093 & $0.0003-1.0$ \\
\hline Sedimentary bedrock & $\mathrm{m} / \mathrm{d}$ & 0.09 & 0.10 & $0.003-1.0$ \\
\hline
\end{tabular}

Table 10. Geologic-unit parameters.

[For the purposes of estimating porosity and vertical anisotropy, thin glacial till and the underlying bedrock were treated as separate units]

\begin{tabular}{|c|c|c|}
\hline Parameter & Porosity & Vertical anisotropy \\
\hline Alluvium & 0.375 & 4 \\
\hline Artificial Fill & 0.375 & 30 \\
\hline Stratified glacial deposits - high conductivity & 0.375 & 4 \\
\hline Stratified glacial deposits - intermediate conductivity & 0.375 & 30 \\
\hline Stratified glacial deposits-low conductivity & 0.375 & 30 \\
\hline Marsh sediments & 0.375 & 1,000 \\
\hline Thick glacial till & 0.25 & 10 \\
\hline Thin glacial till & 0.275 & 10 \\
\hline Igneous bedrock & 0.01 & 1 \\
\hline Metamorphic bedrock & 0.01 & 1 \\
\hline Metamorphic/sedimentary bedrock & 0.01 & 1 \\
\hline Sedimentary bedrock & 0.01 & 1 \\
\hline
\end{tabular}

level in the well by using the simulated water table and the hydraulic head in model layer 4 prior to comparison with the observed water-level altitude. Water-level data for all wells are available in the National Water Information System (U.S. Geological Survey, 2020).

\section{Stream Water-Level Altitude}

In humid regions, the water level in a stream during base-flow conditions is the altitude of the water table adjacent to the stream. If the land surface is relatively flat in the area near a stream, stream water-level altitudes provide information on the water-table altitude across that area. Stream water-level altitudes, as recorded in the NHDPlus version 2.1 (U.S. Environmental Protection Agency and U.S. Geological 
Table 11. Observation groups, weights, and values of the absolute mean residuals.

[Initial mean absolute errors were calculated prior to the scaling of recharge and prior to calibration. Post-calibration mean absolute-error values were calculated after calibration but before the parameter values were rounded to two significant digits. Final mean absolute-error values for the 1,000 -foot (ft) model were calculated after the calibrated parameter values were rounded to two significant digits. m, meter; $\mathrm{m}^{3} / \mathrm{d}$, cubic meter per day]

\begin{tabular}{|c|c|c|c|c|c|c|c|}
\hline Observation group & Number & Weight & Units & $\begin{array}{c}\text { Initial mean } \\
\text { absolute } \\
\text { error }\end{array}$ & $\begin{array}{c}\text { Post- } \\
\text { calibration } \\
\text { mean } \\
\text { absolute } \\
\text { error }\end{array}$ & $\begin{array}{c}\text { Final mean } \\
\text { absolute } \\
\text { error, } \\
1,000-f t \\
\text { model }\end{array}$ & $\begin{array}{c}\text { Final mean } \\
\text { absolute } \\
\text { error, } \\
500-f t \\
\text { model }\end{array}$ \\
\hline Groundwater levels & 65 & 0.6 & $\mathrm{~m}$ & 6.3 & 4.7 & 4.7 & 4.5 \\
\hline Stream altitude & 477 & 0.34 & $\mathrm{~m}$ & 1.1 & 1.1 & 1.1 & 1.3 \\
\hline Flooded land/dry streams & 2 & 200 & Dimensionless & 0.32 & 0.085 & 0.086 & 0.11 \\
\hline
\end{tabular}

Survey, 2012) from flat areas (with a land-surface slope less than or equal to 0.50 percent) were used as calibration targets for the water-table altitude (fig. 8; Barclay and Mullaney, 2021a, b). For each stream reach within a model cell, the simulated water level at the midpoint of the stream reach was compared to the mapped water level in the stream. Streams and rivers within the model area were simulated by using the DRN package, which will cause groundwater to discharge when the head within the cell is higher than the DRN boundary, thereby lowering the head within the cell; however, the DRN package does not affect the simulation results when the head within the cell is lower than the DRN boundary; the observed stream water-level altitudes can increase the simulated groundwater head in those locations where the head is below the DRN boundary.

\section{Streamflows}

Observations of streamflows during base-flow conditions can provide important information regarding the amount of water entering the groundwater system and constrain estimates of groundwater recharge, which can then be used to estimate hydraulic-conductivity values. Because groundwater-flow rates are a function of differences in groundwater head (the driving force for groundwater movement) and hydraulic conductivity (property of the aquifer indicating the ease of flow through the subsurface), the use of both flow rates (refined groundwater-recharge estimates) and observations of groundwater heads (water levels in wells and streams) can help to constrain the calibrated hydraulic conductivities to values typical for the geologic units present in this study area. In this study, mean daily discharge values under base-flow conditions from 14 streamgages with relatively low streamflow alterations caused by groundwater or surface-water withdrawals were used as calibration targets (fig. 7, table 5). Twelve of the 14 streamgages were classified as "Reference" streamgages in the GAGES II dataset (Falcone, 2011) which indicate minimal hydrologic disturbance, and two additional streamgages (01208873, Rooster River at Fairfield, Conn.; 012035055, Pootatuck River at Berkshire) were determined to be relatively unaffected by water use in their drainage areas on the basis of observations during prior site visits. For each streamgage, mean base flow was calculated by using the PART (Rutledge, 1998) and BFI (Gustard and others, 1992) algorithms as implemented in the DVstats package in $\mathrm{R}$ ( $\mathrm{R}$ Core Team, 2019). The mean value from longest continuous period of daily mean streamflow values was used as a calibration target. The lengths of streamflow records ranged from 11 to 30 years, with a mean of 24.5 years, and drainage areas ranged from $10 \mathrm{~km}^{2}$ to $259 \mathrm{~km}^{2}$, with a mean of $61 \mathrm{~km}^{2}$. The streamflow values, both observed and simulated, were normalized for drainage area by the use of a square-root transformation. The square root of the observed base flow at each streamgage was compared with the square root of the total simulated groundwater discharge upstream of the streamgage location. Streamflow data for all gages are available in the National Water Information System (U.S. Geological Survey, 2020).

\section{Flooded Land/Dry Stream Errors}

Specific quantitative hydrologic observations are time-consuming and expensive to collect, particularly for a study that is regional in scope. The available measurements, however, can be supplemented with qualitative observations based on the assumptions that in humid areas such as New England, perennial streams typically contain water (which means that the altitude of the water table is above the altitude of the streambed) and that the land surface is dry (which means that the altitude of the water table is below the land-surface altitude). In combination, these observations constrain the location of the simulated water table as between the streambed and the surrounding land surface. In this study, two error rates were calculated: (1) the percentage of river cells that were dry (because the water table was more than $1 \mathrm{~m}$ below the stream-bottom altitude) and (2) the percentage of non-wetland terrestrial cells that were flooded (because the water table was more than $1 \mathrm{~m}$ above the land surface) (Starn and Belitz, 2018). The calibration observations were (1) the difference between the error rates was equal to zero, which prevents the calibration software from minimizing one error 
rate while increasing another; and (2) the sum of the error rates was equal to zero, which directs the calibration software to minimize both values. Although the dry-streams component of this error metric overlaps the stream water-level-altitude observations, it contributes to the calibration targets in two ways. First, it includes more stream reaches than are included in the stream water-level-altitude observations because it does not filter out stream reaches with greater slopes. Second, the dry-stream metric is explicitly balanced with the flooded-land metric (by using the sum and the difference of the two metrics rather than the metrics themselves).

\section{Calibration Results}

The calibration process involved iteratively adjusting the values of the model parameters, calculating simulated values of the observations, comparing the simulated and observed values, and readjusting the parameter values. In total, the calibration process required seven iterations. The initial values of the mean absolute error were calculated by using the uncalibrated model prior to scaling the groundwater recharge. These values serve as a baseline for quantifying the model improvement with calibration. The greatest relative decreases in the observation group's mean absolute residual were in the streamflow $\left(23,000\right.$ to $\left.7,200 \mathrm{~m}^{3} / \mathrm{d}\right)$ and the flooded-land/drystreams metrics $(0.32$ to 0.11$)$ (table 11$)$. The mean absolute errors of the stream water-level altitudes changed relatively little during the calibration process. The model fit was most sensitive to the values for the alluvium, glacial till/bedrock complex, metamorphic bedrock, stratified glacial deposits (high conductivity), and sedimentary bedrock. The model fit was least sensitive to the values for igneous bedrock, the combined metamorphic-and-sedimentary bedrock unit, marsh sediments, and artificial fill.

\section{Post-Calibration Model Adjustments}

After calibration, two adjustments were made to the model. First, the final parameter values were rounded to two significant digits. The result was the final 304.8-m (1,000-ft) model, and the mean absolute residuals for the final model did not differ substantially from the mean absolute residuals for the post-calibration 304.8-m (1,000-ft) model, which did not have rounded parameter values (table 11). Second, the horizontal discretization was reduced from $304.8 \mathrm{~m} \mathrm{(1,000 \textrm {ft } )}$ to $152.4 \mathrm{~m} \mathrm{(500} \mathrm{ft)} \mathrm{with} 1,001,952$ cells in each layer, of which 399,976 were active, to better represent local patterns in groundwater discharge. This reduced discretization created the final 152.4-m (500-ft) model. ${ }^{4}$ The refinement was done after calibration to reduce computational needs during calibration. The mean absolute residuals for the final model with a refined 152.4-m (500-ft) grid did not vary substantially from the mean absolute residuals for the final model with the original

\footnotetext{
${ }^{4}$ The implications of model-grid resolutions are discussed in the subsequent section, "Grid Resolution."
}

304.8-m (1,000-ft) grid; for this reason, the final 152.4-m (500-ft) model was used for all calculations except as noted in the text.

\section{Comparison of Observations and Simulated Equivalents}

The simulated values from the final $152.4-\mathrm{m}(500-\mathrm{ft})$ model for three of the observation groups were compared with the corresponding observations (fig. 15). Residuals - the differences between the observed and simulated values for groundwater levels - ranged from - 11 to $19 \mathrm{~m}$ (fig. 16A), with a mean residual of $0.6 \mathrm{~m}$ and a mean absolute residual of $4.5 \mathrm{~m}$ (table 11). A positive mean residual indicates that the model has a bias for underpredicting groundwater levels. The magnitudes of the residuals were larger for wells located at higher altitudes.

Residuals for stream altitudes ranged from -2.8 to $37 \mathrm{~m}$, with a mean residual of $1.2 \mathrm{~m}$ and a mean absolute residual of $1.3 \mathrm{~m}$ (table 11, fig. 16B). A positive mean residual indicates that the model has a bias for underpredicting the stream waterlevel altitude (fig. 15B). Most of the simulated stream altitudes (81 percent) were within $2 \mathrm{~m}$ of the observed altitude.

Residuals for base-flow discharge ranged from 170 to $17,000 \mathrm{~m}^{3} / \mathrm{d}\left(0.07\right.$ to $\left.6.9 \mathrm{ft}^{3} / \mathrm{s}\right)$, with a mean residual of $7,200 \mathrm{~m}^{3} / \mathrm{d}\left(2.9 \mathrm{ft}^{3} / \mathrm{d}\right)$ and a mean absolute residual of $7,200 \mathrm{~m}^{3} / \mathrm{d}\left(2.9 \mathrm{ft}^{3} / \mathrm{s}\right)$ (table 11, fig. 17). The errors ranged from 0.25 to 22 percent of observed streamflows, with a mean of 13 percent. A positive mean residual indicates that the model has a bias for underpredicting base flow.

Among non-wetland terrestrial model cells, 11 percent were flooded; the simulated water level was above the land surface (fig. 18). The largest clusters of flooded terrestrial cells were in New York and in isolated areas near the model boundary. In highly urbanized areas, groundwater recharge may be overestimated because of the presence of storm drains and other types of water infrastructure that are not simulated in the model. Urban water dynamics are further discussed in the section "Limitations and Factors Affecting Model Simulations" below. Of the model cells that included stream segments or coastal water, 10 percent were dry. Most of the dry streams were in upland areas and along minor tributaries. Because perennial streams were used for the stream network in the model, dry streams were assumed to be errors.

\section{Particle-Tracking Model Development}

Particle-tracking software can be used in conjunction with a groundwater-flow model to determine flow directions and rates within an aquifer. Hypothetical particles representing packets of water are tracked through the aquifer on the basis of the simulated groundwater flow and a user-specified distribution of subsurface porosity. The resulting particle-tracking analysis provides information about contributing areas, flowpath depth, and subsurface travel time. 

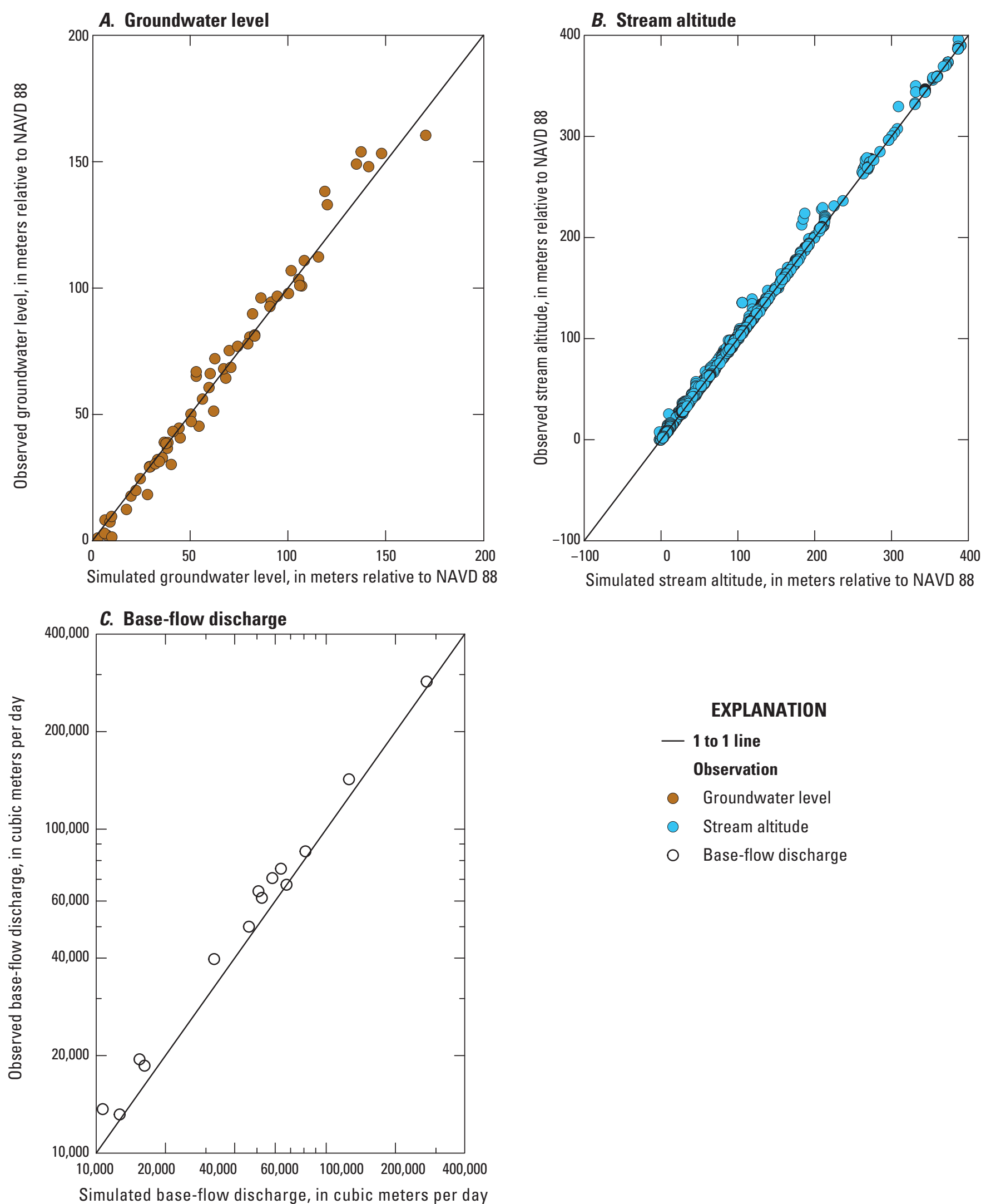

\section{EXPLANATION}

- 1 to 1 line

Observation

- Groundwater level

- Stream altitude

O Base-flow discharge

Figure 15. A, observed and simulated groundwater levels, $B$, observed and simulated stream altitudes, and $C$, observed and simulated base-flow discharges, north shore of Long Island Sound (coastal Connecticut and adjacent areas of New York and Rhode Island). NAVD 88, North American Vertical Datum of 1988. 


\section{A. Groundwater-level residuals}

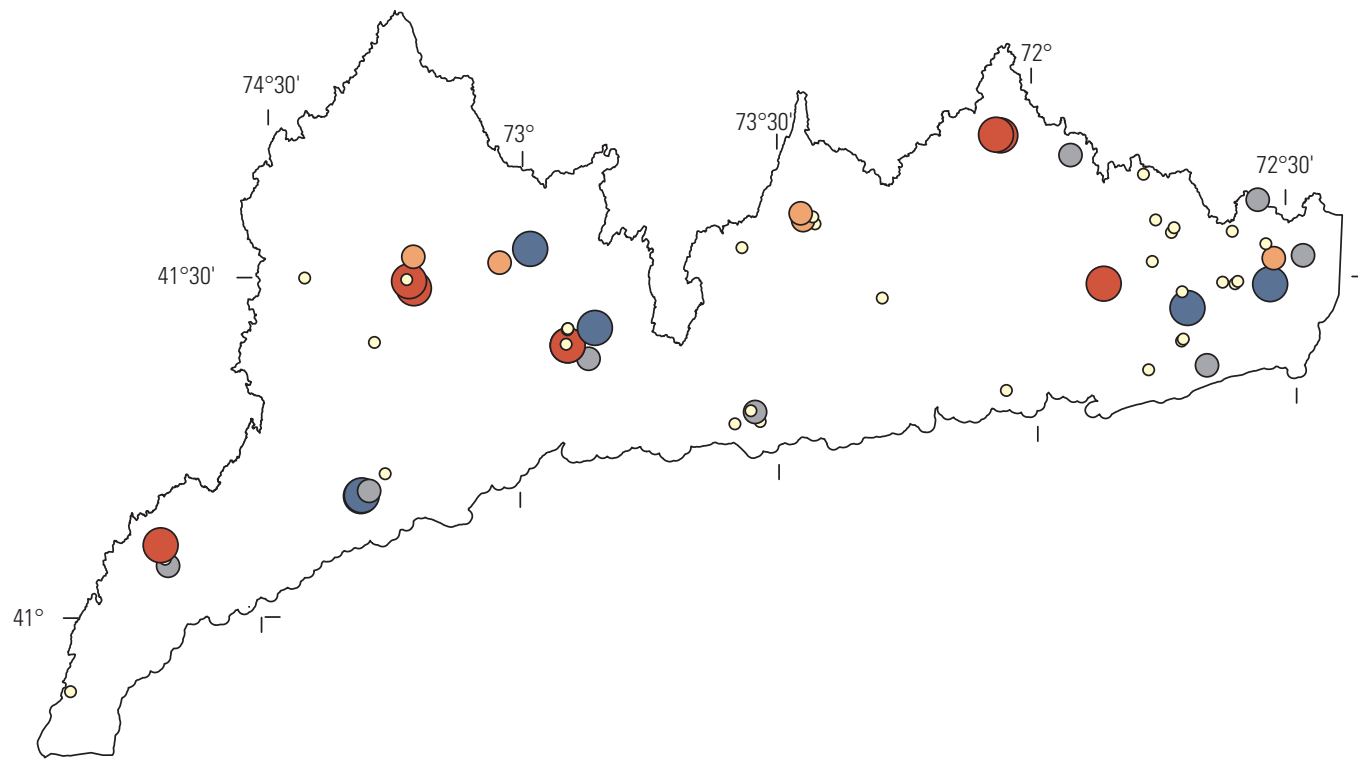

EXPLANATION

Sym Study area

Groundwater-level residual, in meters

$\leq-8.0$

-7.9 to -3.0

-2.9 to 3.0

3.1 to 8.0

\section{B. Stream-altitude residuals}

EXPLANATION

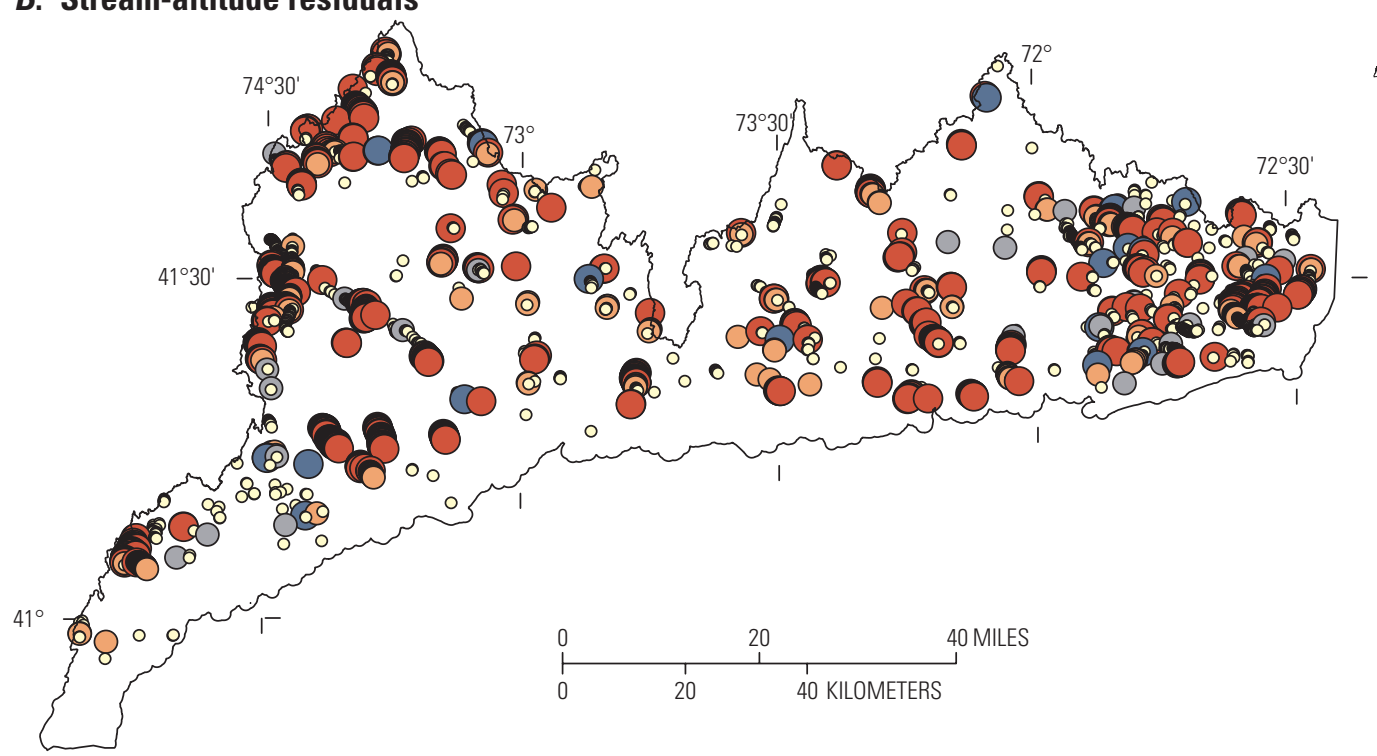

\section{Sum Study area \\ Stream-altitude residual,} in meters

$\leq-1.0$

-0.9 to -0.5

-0.4 to 0.5

0.6 to 1.0

$>1.0$

\section{Connecticut State Plane coordinate system} North American Datum of 1983

Figure 16. $A$, groundwater-level residuals and $B$, stream-altitude residuals on the north shore of Long Island Sound (coastal Connecticut and adjacent areas of New York and Rhode Island).

In this study, MODPATH (Pollock, 2012) was used for the particle-tracking simulation. A total of 3 million particles was released; the number of particles within each active cell was proportional to the calibrated groundwater-recharge rate; and all particles were distributed in a grid formation across the top face of the model cells in the upper-most non-dry grid layer. Porosity values were based on geologic material (table 10) (Lyford and others, 2007). Particles were released at a single predetermined time, tracked in the forward direction, and allowed to pass through weak sinks. ${ }^{5}$ Some particles did not move during the simulation or were discharged immediately because they were released in an area of groundwater discharge.

\footnotetext{
${ }^{5}$ Weak sinks occur in model cells where some, but not all, water flowing into the cell leaves the study area. For example, a well with a pumping rate less than the flux of water into the cell would be a weak sink.
} 


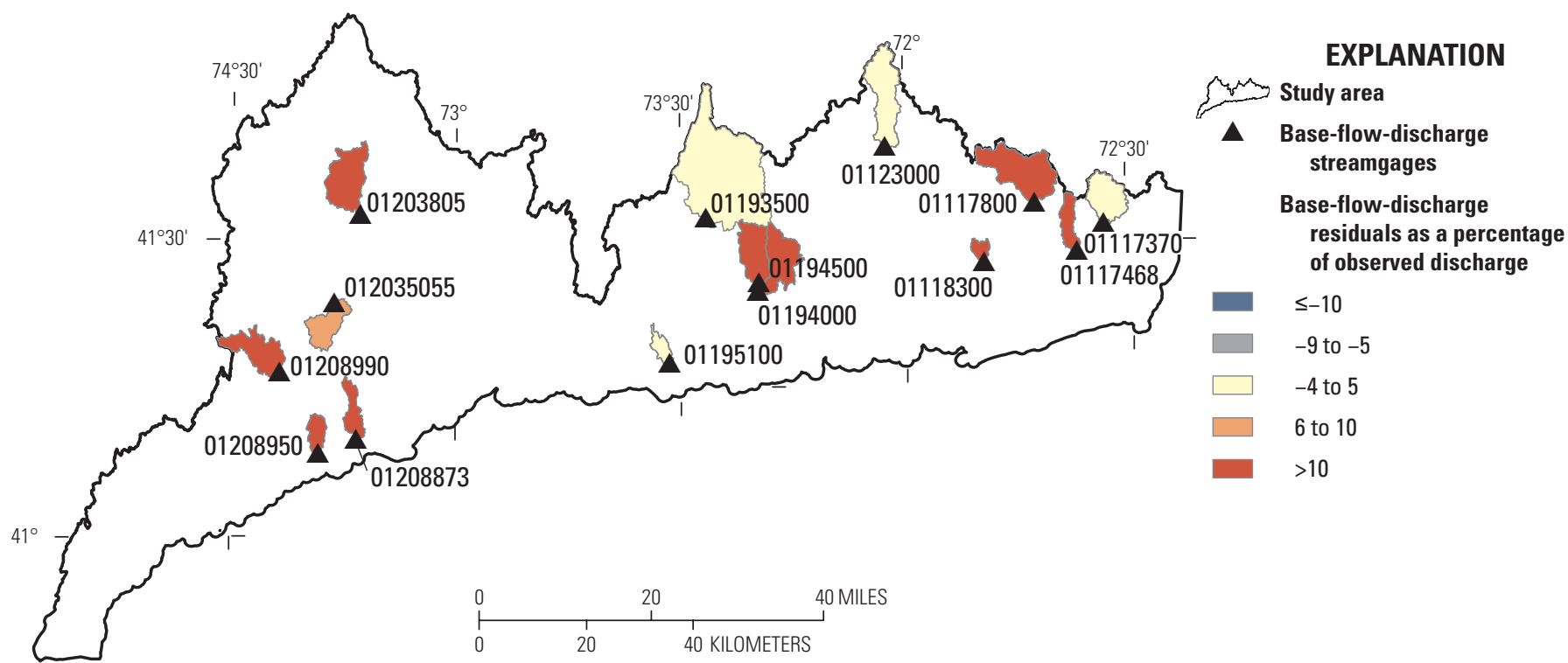

2011 Connecticut State Plane coordinate system North American Datum of 1983

Figure 17. Base-flow-discharge residuals as a percentage of observed base flow on the north shore of Long Island Sound (coastal Connecticut and adjacent areas of New York and Rhode Island). The observed values are measured at the gages; the residuals are mapped to the watershed areas that contribute groundwater discharge to the simulated base flow values.

\section{Groundwater Budgets and Travel Times}

Groundwater budgets describe the inflows and outflows of groundwater for a given area, and for this reason, they are an important tool in describing the groundwater-flow system. Major components of a groundwater budget include inflows such as natural-recharge and septic-return flows; outflows such as well withdrawals, discharges to streams, rivers, and coastal waters; and the movement of groundwater to or from adjoining areas.

The time required for water to travel through the groundwater system from recharge to discharge areas also provides important information about the hydrologic system. Groundwater travel times provide insight into how water moves through an aquifer system, which in turn affects the transport and chemical reactions of nutrients and other contaminants (McGuire and McDonnell, 2006). For example, longer travel times could allow for the reduction of more nitrogen or a longer required time for flushing legacy nitrogen out of the groundwater system. Because groundwater travel times can influence the time delay between management actions and visible effects, these travel times might be an important factor in water-resource-management plans (Meals and others, 2010).

Groundwater budgets and travel times were compiled for the entire study area, for each HUC12 watershed and its adjacent coastal waters (extended HUC12s) throughout the study area, and for 14 priority embayments selected by CT DEEP (Connecticut Department of Energy and Environmental Protection, 2019a) (fig. 2). Because the HUC12 boundaries end at the shoreline, groundwater discharge to adjacent coastal waters would be counted as lateral transfers out of the basin. To include adjacent coastal waters in the calculation of groundwater budgets and travel times, coastal waters seaward of the HUC12 boundaries, between the edge of land and the offshore model boundary, were assigned to the nearest HUC12, as indicated in fig. 12 for a subset of HUC12 watersheds. The extended HUC12 watersheds were used for the HUC12 groundwater-budget and travel-time calculations.

\section{Groundwater Budgets}

Across the entire study area, inflows were predominantly from natural recharge (98.8 percent), with a small internally recycled contribution from septic return flows (1.2 percent) (Barclay and Mullaney, 2021b). Outflows consisted of discharge to inland waters (90.6 percent), discharge to coastal waters ( 7.0 percent), and well withdrawals (2.4 percent). The relative magnitudes of components of the groundwater budget varied widely across HUC12 and embayment watersheds. Groundwater-budget data are available in Barclay and Mullaney (2021b).

\section{HUC12 Groundwater Budgets}

Natural recharge accounted for 70 to 98 percent of the inflows into HUC12 watersheds, with a median of 94 percent (fig. 19) (Barclay and Mullaney, 2021b). In HUC12 watersheds with septic return flows, these flows accounted 
A. Flooded land

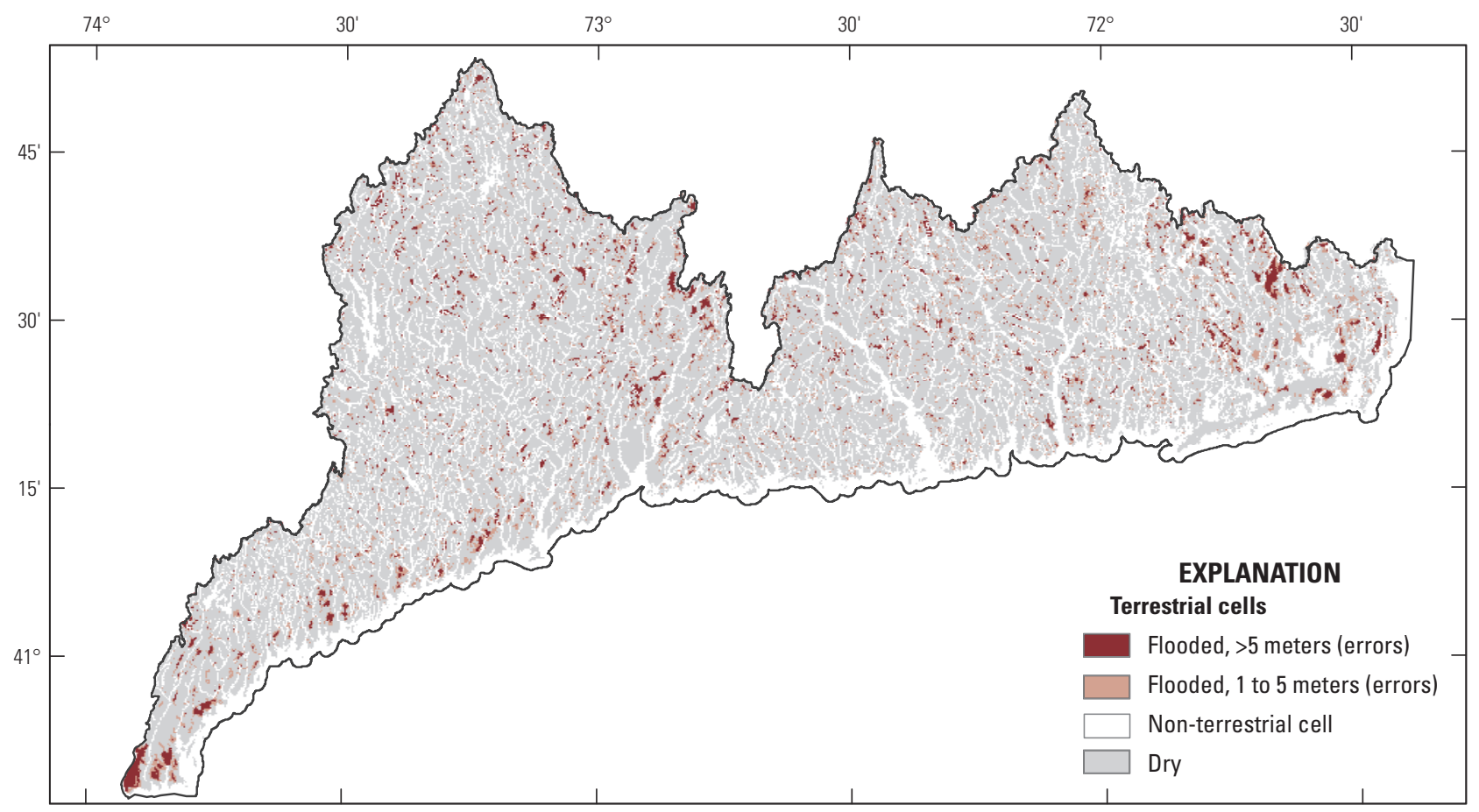

B. Dry streams

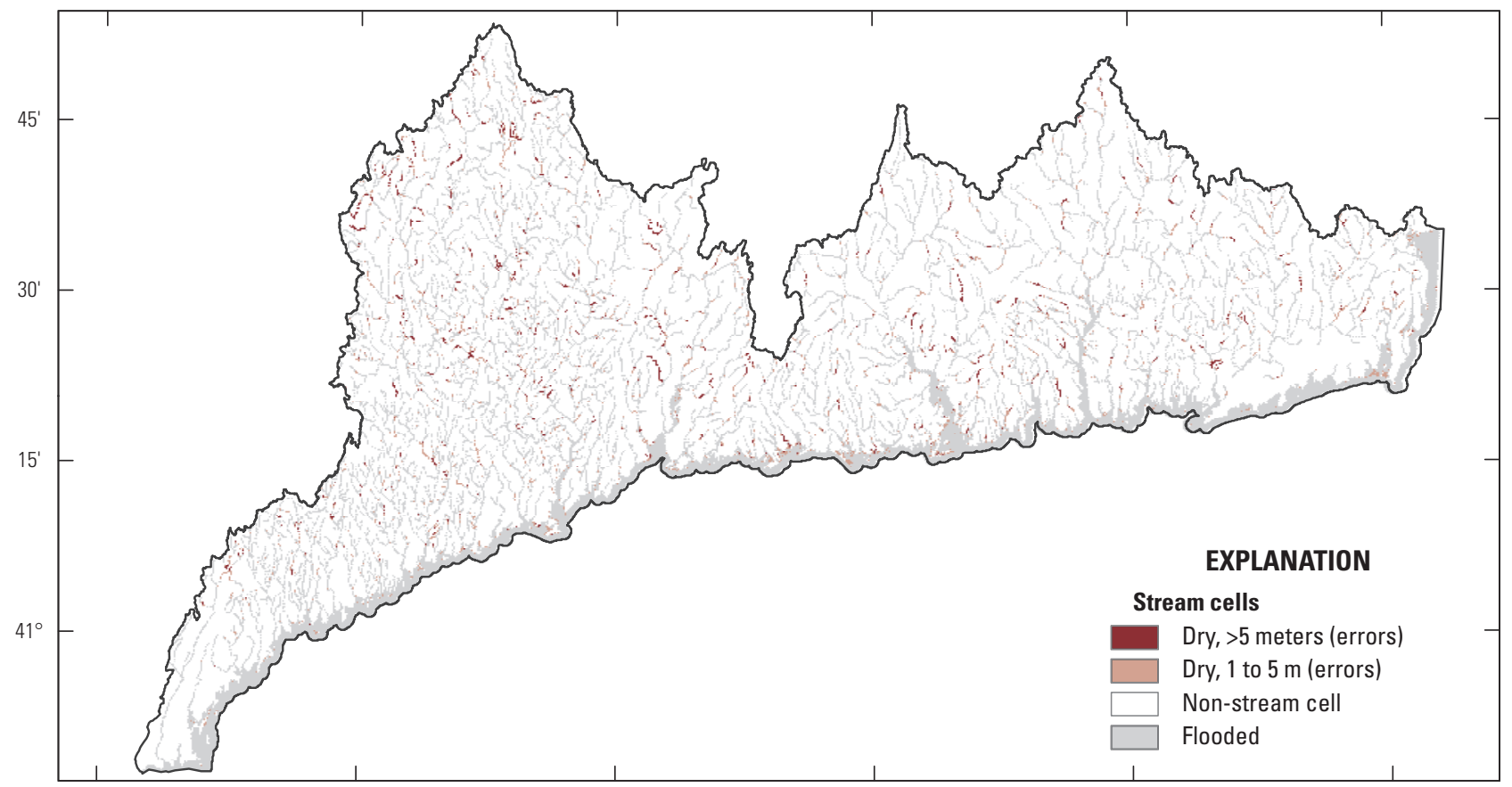

2011 Connecticut State Plane coordinate system North American Datum of 1983

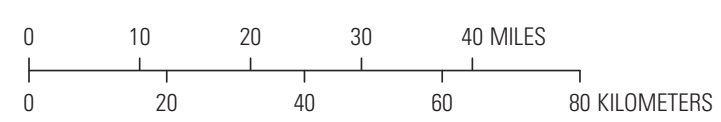

Figure 18. A, flooded land and $B$, dry streams with specified ranges of errors on the north shore of Long Island Sound (coastal Connecticut and adjacent areas of New York and Rhode Island). Errors are shown in two groups-the depth of flooding or the depth to water below the stream. 
for less than 1 to 4 percent of the groundwater inflows into HUC12 watersheds, with a median of 0.9 percent. Septic return flows are predominantly internally recycled water from well withdrawals and tend to account for larger fractions of the groundwater budget near, but not necessarily in, HUC12 watersheds with high population densities (fig. 19B). Transfers of groundwater into the HUC12 watersheds from nearby watersheds account for 2 to 29 percent of inflows, with a median of 5 percent (fig. 20A). Transfers of groundwater from nearby watersheds were most common near the coast and near the Housatonic, Thames, and Pawcatuck Rivers.

Groundwater discharge to inland waters ranged from 4 to 98 percent, with a median of 91 percent, of the total discharges of groundwater within each HUC12 (fig. 21). Groundwater discharged to coastal waters from 52 HUC12 watersheds, in which it ranged from less than 1 to 66 percent of groundwater outflows, with a median of 13 percent. The ratio of inland to coastal discharge varied widely among HUC12 watersheds that discharged to coastal waters, from less than 1 to more than 1,000, with a median of 6 . In most HUC12 watersheds, even those that border coastal waters, outflows are dominated by discharge to inland waters. This ratio suggests that most groundwater-transported nitrogen will discharge first to inland waters, where it may be further attenuated by biological uptake and denitrification (Helton and others, 2011; Mulholland and others, 2008; Reisinger and others, 2015; Wollheim and others, 2017), prior to discharge to coastal waters. It does not, however, suggest substantial additional delays between discharge from the groundwater system and arrival to coastal waters, as transport within surface water is relatively fast compared to groundwater. In two watersheds, Westchester Creek-East River (map no. =1) and East CreekMamaroneck Harbor (map no. = 5) (figs. 2 and 22A) (Barclay and Mullaney, 2021b), outflows are dominated by discharge to coastal waters, as indicated by ratios of inland to coastal discharge of 0.5 or less. In HUC12 watersheds with simulated well withdrawals, the percentages of outflows to wells ranged from less than 1 to 14 percent, with a median of 1 percent. As with septic return flows, well withdrawals were greatest near, but not necessarily in, watersheds with higher population densities (fig. 22B). Well withdrawals are particularly important in the Mill River (map no. =53), Headwaters Quinnipiac River (map no. = 55), Pattagansett River-Frontal Long Island Sound (map no. = 78), Lower Pawcatuck River (map no. = 98), and Chipuxet River-Pawcatuck River (map no. = 107), where they account for more than 7 percent of groundwater outflows (figs. 2 and 22B). Lateral transfers of groundwater to other HUC12 watersheds ranged from 2 to 41 percent of outflows, with a median of 5 percent. Groundwater transfers are a substantial component of the groundwater budget in Westchester Creek-East River (map no. $=1 ; 41$ percent of outflows), and Chipuxet River-Pawcatuck River (map no. $=107$; 26 percent of outflows) (figs. 2 and 20B). Lateral transfers of groundwater are indicative of groundwater watersheds that are not coincident with topographic (surface-water) watersheds, or of areas with low topographic relief for which topographic boundaries may not be precisely represented in a regional model. Transfers of groundwater out to nearby watersheds were most common near the coast and near the Housatonic and Pawcatuck Rivers.

\section{Embayment Groundwater Budgets}

Natural recharge accounted for 75 to 98 percent, with a median of 93 percent, of the groundwater inflows into the priority embayment watersheds (fig. 2) (Barclay and Mullaney, 2021b). Priority embayments were identified by CT DEEP (2019a) as focus areas for nitrogen management. Septic return flows occurred in 12 embayment watersheds, where it accounted for 0.1 to 3 percent of the groundwater inflows into the embayment watersheds, with a mean of 0.7 percent. Transfers of groundwater into the embayment watersheds from nearby watersheds account for 2 to 24 percent of inflows, with a median of 5 percent. Large transfers of groundwater from or to adjacent watersheds could indicate areas where the groundwater divides differ from the surface-watershed divides, or they could be a modeling artifact due to a combination of low topographic relief and coarse model resolution.

Groundwater discharge to inland waters accounted for 12 to 91 percent of outflows from priority-embayment watersheds, with a median of 74 percent (fig. 2) (Barclay and Mullaney, 2021b). Groundwater discharge to coastal waters ranged from 2 to 56 percent, with a median of 15 percent. Groundwater withdrawals were present in 12 embayment watersheds, where they accounted for 0.5 to 14 percent of total outflows, with a median of 1 percent. Transfers of groundwater to other basins ranged from 2 to 33 percent of outflows, with a median of 10 percent.

\section{Groundwater Travel Times}

Across the study area, the interquartile range of groundwater travel times to all waters, both coastal and inland, extended from 0.1 to 5.9 years, with a median of 1.9 years (fig. 23A) (Barclay and Mullaney, 2021b). Travel times for water discharging to inland waters were similar, with an interquartile range of 0.1 to 5.7 years and a median of 1.9 years (fig. 23B). Travel times for water discharging to coastal waters had a similar median, but a broader range, with an interquartile range of 0 to 11.1 years and a median of 1.9 years (fig. 23C). Because groundwater that discharges to inland waters will be transported to coastal waters without substantial additional delays, in areas with longer travels times for groundwater discharge to coastal waters, groundwater discharge to inland surface-water networks (streams and rivers) might shortcircuit transport to coastal waters as compared to groundwater transport directly to coastal waters. Travel times tended to be slightly longer in lower elevation and coastal areas, where groundwater-table gradients likely are less steep (fig. 24). Groundwater travel-time data are available in Barclay and Mullaney (2021b). 
A. Natural recharge
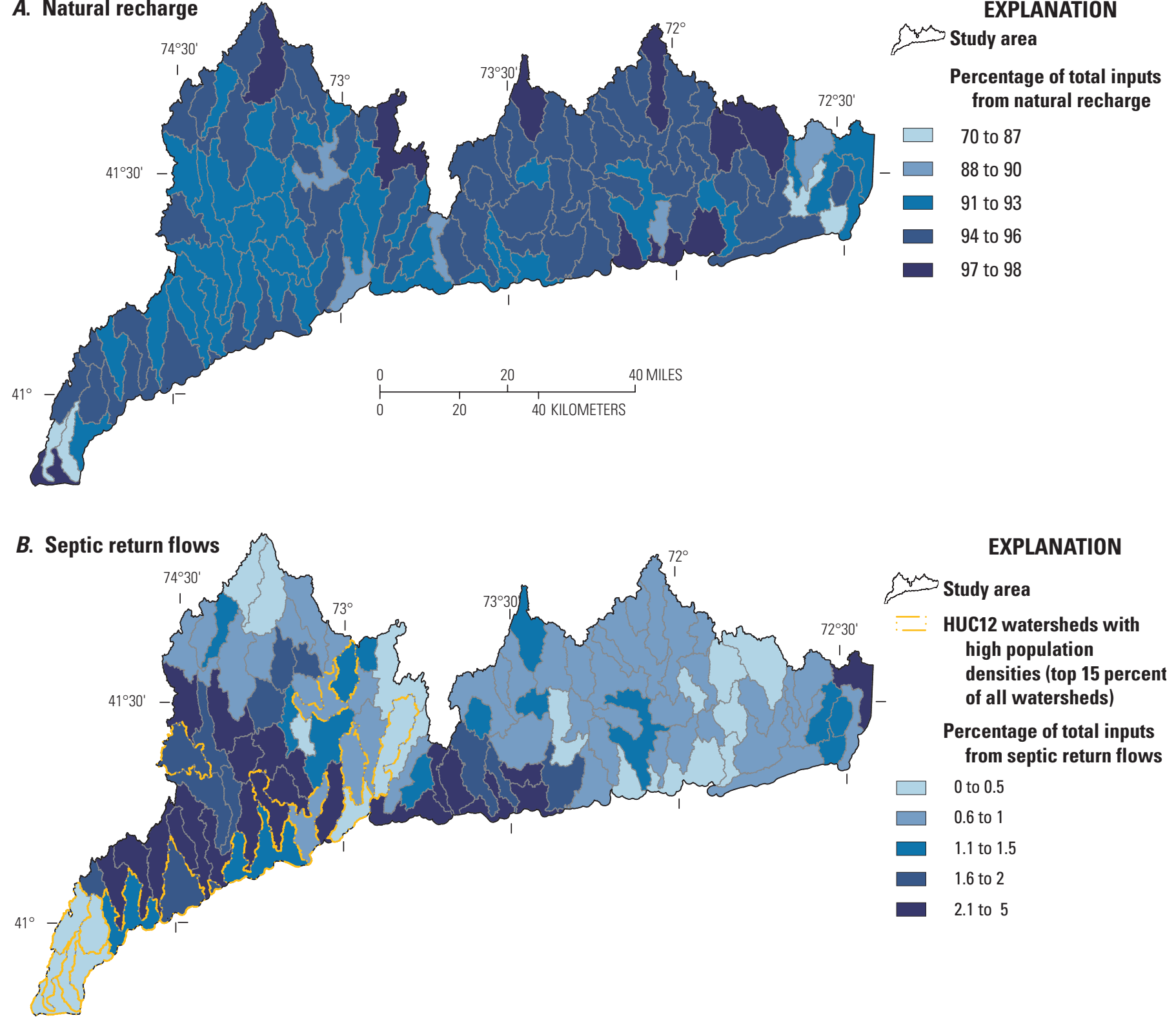

2011 Connecticut State Plane coordinate system

North American Datum of 1983

Figure 19. Percentages of inflows to the groundwater system for each extended HUC12 watershed from $A$, natural recharge and $B$, septic return flows on the north shore of Long Island Sound (coastal Connecticut and adjacent areas of New York and Rhode Island).

\section{HUC12 Groundwater Travel Times}

The median groundwater travel time by HUC12 watershed ranged from 0.9 to 53.5 years, with a median of 1.8 years (fig. 25). Because most groundwater discharges to inland rather than coastal waters, median travel times to inland surface waters, which ranged from 1.1 to 80.2 years with a median of 1.9 years across extended HUC12 watersheds, were similar to travel times to all surface waters. For most extended HUC12 watersheds with groundwater discharge to coastal waters, median travel times to coastal waters were slightly longer, ranging from less than 1 to 61.6 years with a median of 2.8 years across the HUC12 watersheds. In some HUC12 watersheds, the calculated median travel time was 0 years, which was due to a modeling artifact that created spatially extensive areas of discharge. In these watersheds, more than half of the particles (simulated packets of water) in the tracking simulation started in cells with groundwater discharge and consequently discharged immediately with a simulated travel time of 0 days. HUC12 watersheds with particularly long median travel times (greater than 25 years) included Westchester Creek-East River (map no. $=1 ; 31.4$ years); 
A. Lateral transfers of groundwater into the watershed

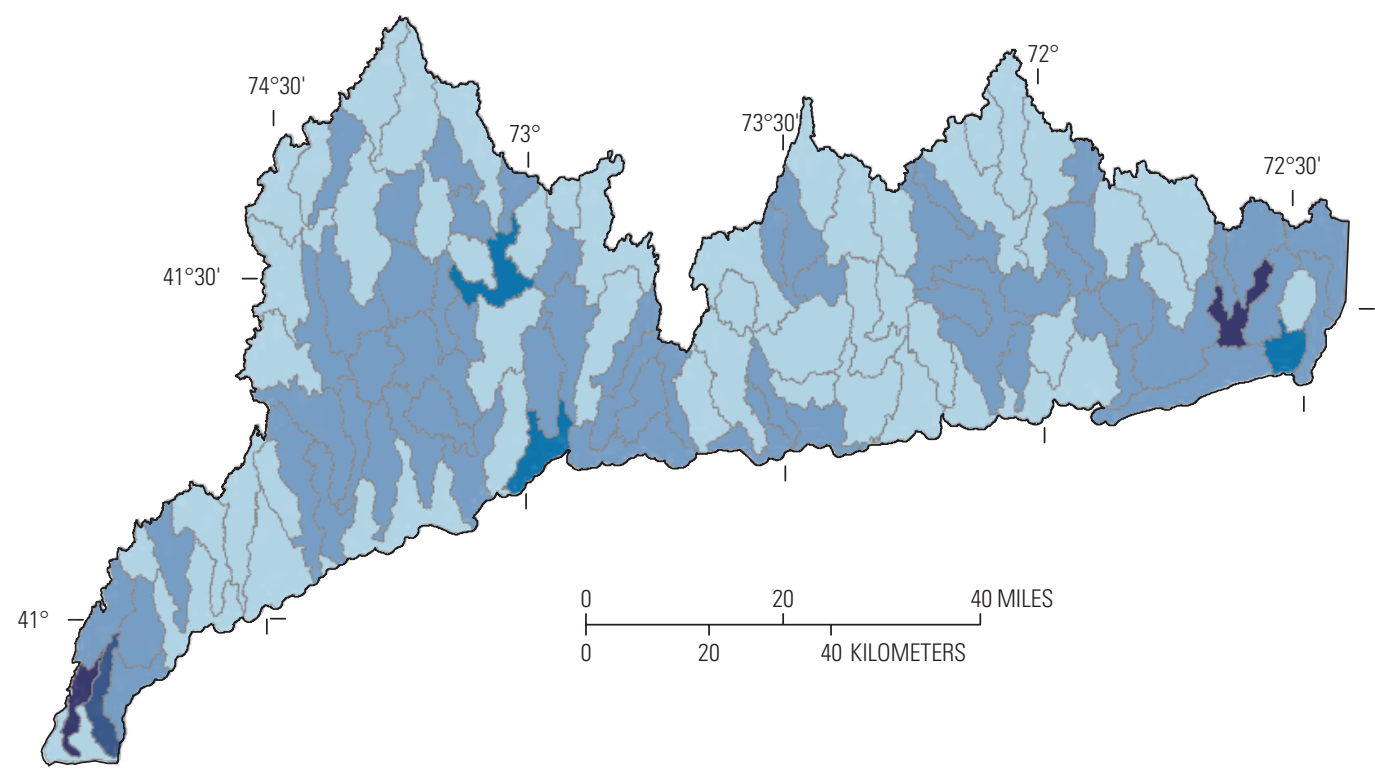

EXPLANATION

Study area

Percentage of total groundwater inflows transferred in laterally

2 to 5

6 to 10

11 to 15

16 to 20

$>20$

\section{B. Lateral transfers of groundwater out of the watershed}

\section{EXPLANATION}

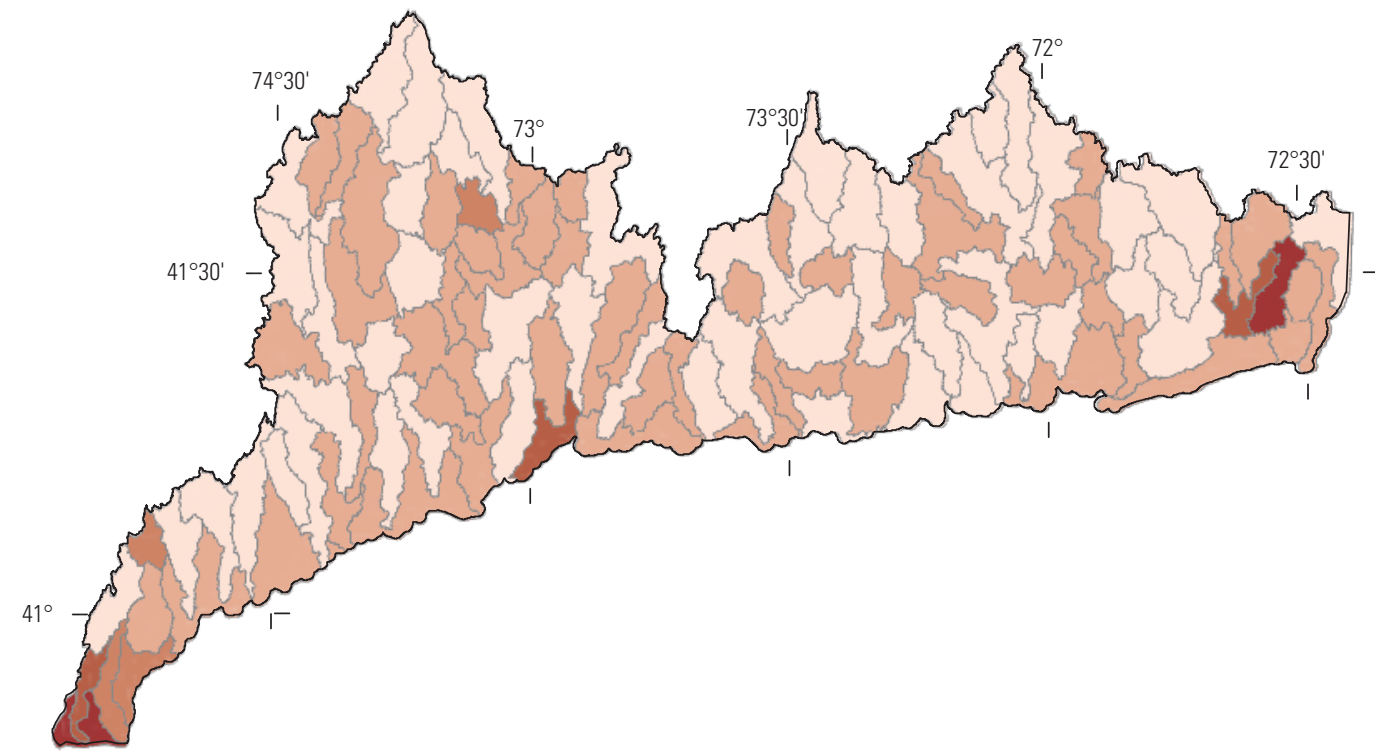

Study area

Percentage of total discharge transferred out laterally

2011 Connecticut State Plane coordinate system North American Datum of 1983

Figure 20. Percentages of $A$, inflows into and $B$, outflows from the groundwater system by lateral transfers of water for each extended HUC12 watershed on the north shore of Long Island Sound (coastal Connecticut and adjacent areas of New York and Rhode Island).

Hutchinson River-Eastchester Bay (map no. $=3 ; 50.3$ years); and East Creek-Mamaroneck Harbor (map no. $=5 ; 53.5$ years) (figs. 2 and 25) (Barclay and Mullaney, 2021b). All three HUC12 watersheds with long travel times are in the highly developed area near New York City. These watersheds contain extensive areas of impervious cover and subsurface water infrastructure, both of which are not well represented in this model. Impervious cover and subsurface infrastructure alter hydrologic flowpaths and can reduce or increase groundwater recharge (Hibbs and Sharp, 2012; Lerner, 1990; Lerner, 2002). These alterations likely influence the simulated travel times. Precise estimation of groundwater travel times in highly urbanized watersheds within the study area would require more extensive data on urban water infrastructure and a more refined assessment of groundwater recharge. 


\title{
A. Groundwater discharge to inland waters
}

EXPLANATION

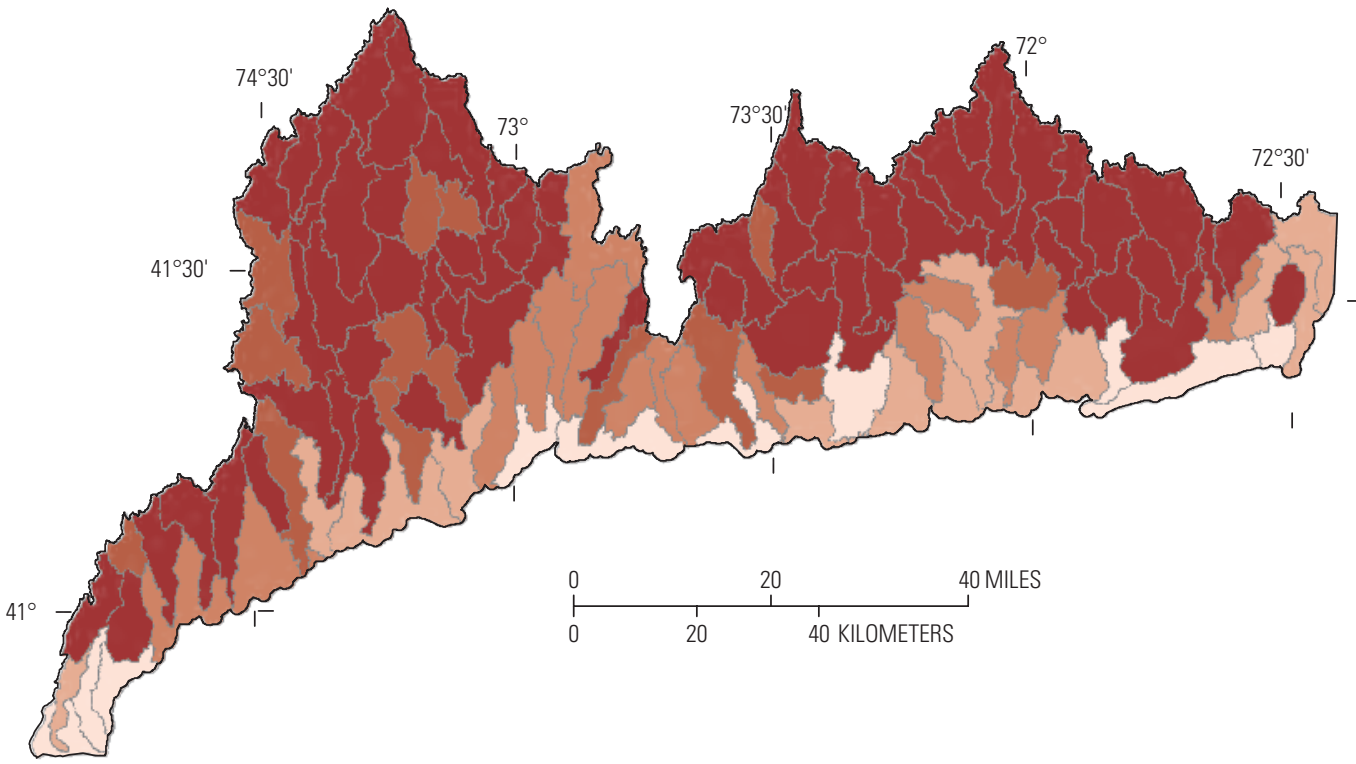

\author{
Sum Study area \\ Percentage of total \\ discharge to
}

inland waters

4 to 50

51 to 75

76 to 85

86 to 90

91 to 100

\section{B. Goundwater discharge to coastal waters}

EXPLANATION

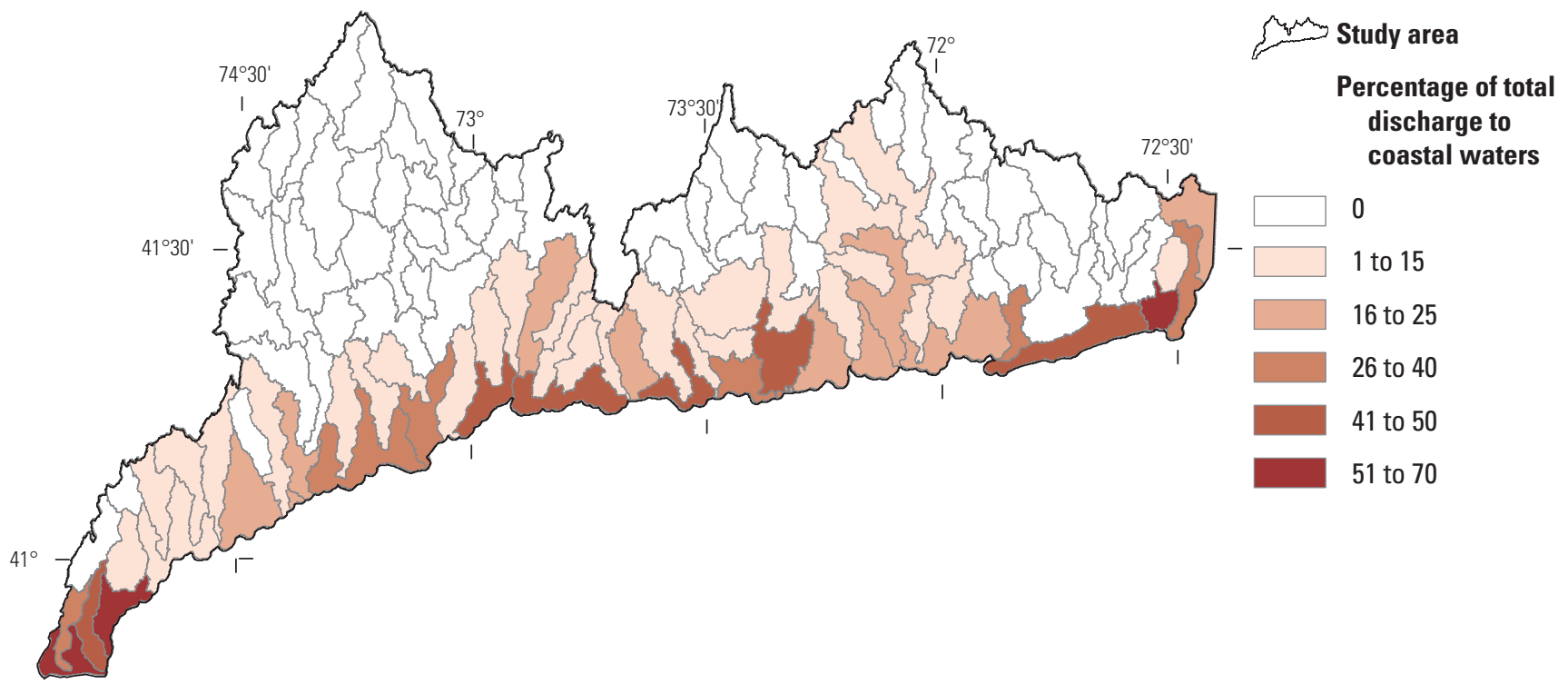

2011 Connecticut State Plane coordinate system

North American Datum of 1983

Figure 21. Percentage of groundwater discharge from each extended HUC12 watershed to $A$, inland water and $B$, coastal waters on the north shore of Long Island Sound (coastal Connecticut and adjacent areas of New York and Rhode Island).

\section{Embayment Groundwater Travel Times}

The median groundwater travel time by priorityembayment watershed ranged from less than 1 to 4.2 years with a median of 1.6 years. Three embayment watersheds had particularly short median travel times (less than 1 year): Page's Cove, Conn. (0 years); Quanaduck Cove, Conn. (0 years); and Little Narragansett Bay, Conn. (0 years) (Barclay and Mullaney, 2021b). Travel times to inland waters ranged from less than 1 year to 4.5 years, with a median of 1.6 years. Travel times to coastal waters were slightly longer, ranging from less than 1 to 18.6 years, with a median of 2.3 years. Median travel times of 0 years are unrealistically short and result from an artifact in the modeling process that overestimates areas of groundwater discharge. A finer resolution grid may be needed to constrain the spatial extent of groundwater discharge and to generate realistic travel-time estimates in these areas. 
A. Groundwater discharge to inland waters versus coastal waters

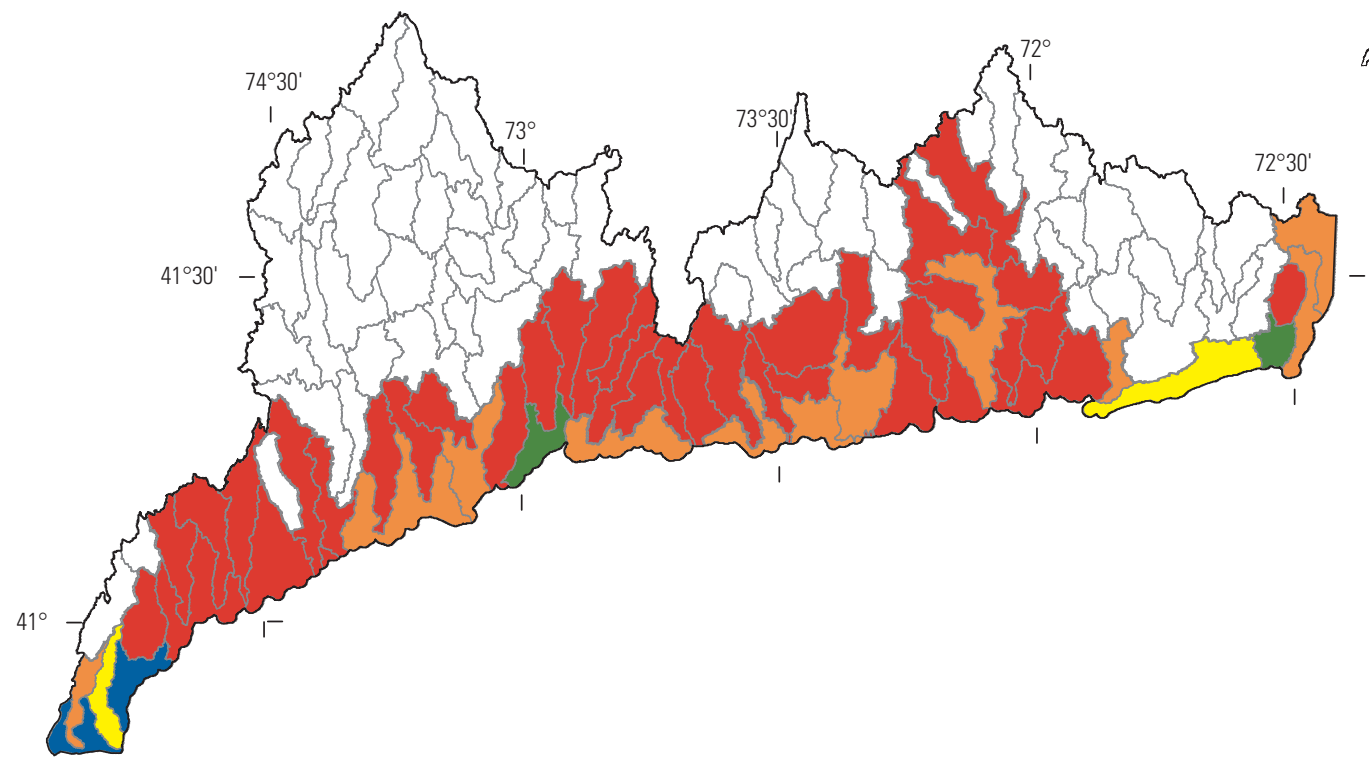

EXPLANATION

Study area

Ratio of discharge to inland versus coastal waters

$\square$ No coastal discharge

$\leq 0.50$

0.51 to 0.90

0.91 to 1.10

1.11 to 3.00

$>3.00$

\section{B. Groundwater discharge to wells}

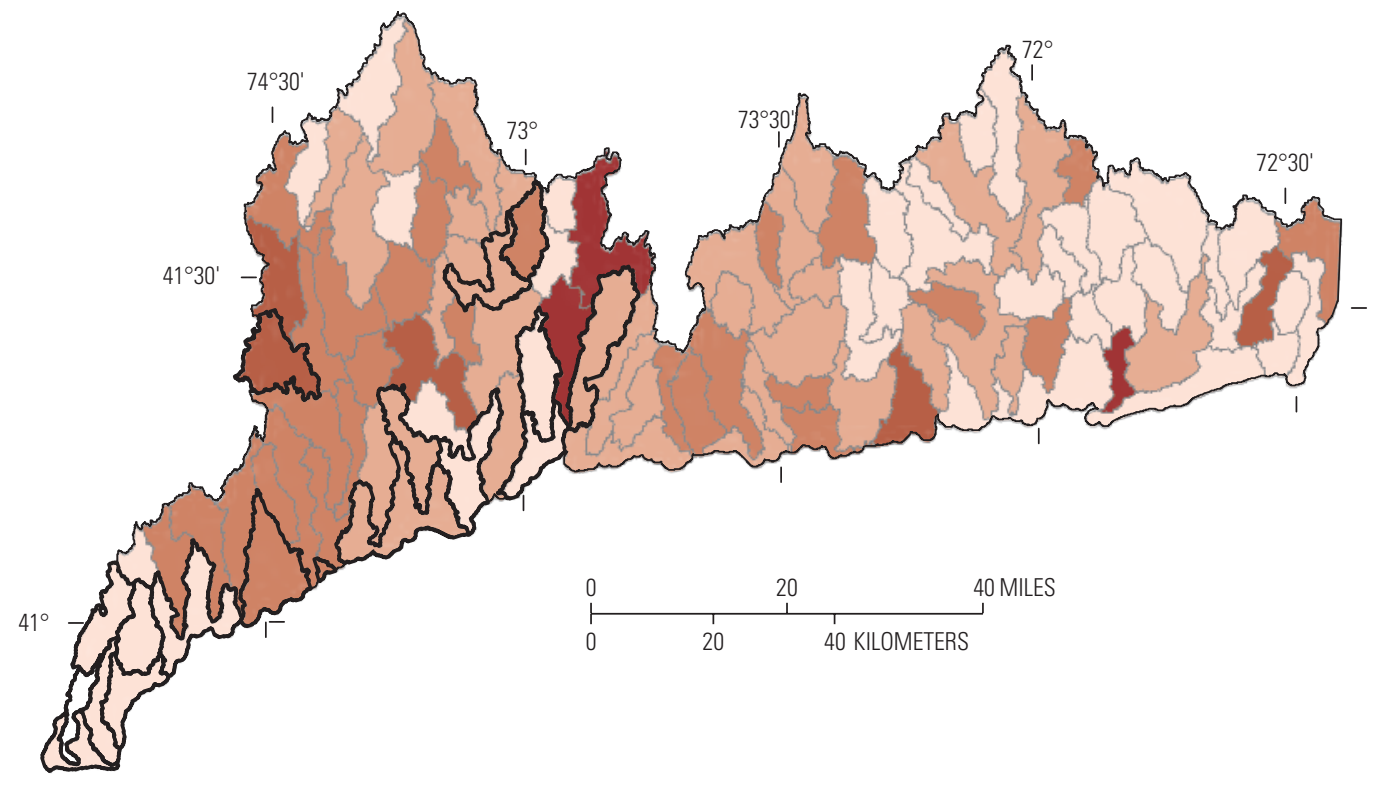

EXPLANATION

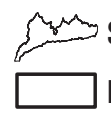

Study area

HUC12 watersheds with high population densities (top 15 percent of all watersheds)

Percentage of total discharge to wells

\section{0}

$>0$ to 1

2

3 to 5

6 to 10

$>10$

2011 Connecticut State Plane coordinate system

Figure 22. A, ratio of groundwater discharge to inland waters to groundwater discharge to coastal waters for each extended HUC12 watershed with groundwater discharge to coastal waters and $B$, percentage of groundwater discharge from each extended HUC12 watershed to wells on the north shore of Long Island Sound (coastal Connecticut and adjacent areas of New York and Rhode Island). 


\section{Contributing Areas of Groundwater Discharge to Coastal and Inland Waters}

Across the study area, most groundwater discharges to surface waterbodies or receptors near the recharge location and does not travel substantial distances. The distance between the recharge and discharge locations ranged from less than $1 \mathrm{~m}$ to more than $12 \mathrm{~km}$, with an interquartile range of 19 to $510 \mathrm{~m}$ and a median of $180 \mathrm{~m}$. Short travel distances between recharge and discharge locations, combined with short travel times suggest that local, as opposed to regional, groundwater flowpaths are dominant in the study area. Most of the land within the study area contributes to groundwater discharge to inland waters (fig. 24), with only $710 \mathrm{~km}^{2}$, or 8 percent of the study area, contributing to coastal waters, a finding that is consistent with the groundwater-budget results that most groundwater within the study area discharges to inland waters prior to reaching coastal waters. Nitrogen that discharges first to inland waters can be attenuated through instream processes such as biological uptake and denitrification (Helton and others, 2011; Mulholland and others, 2008; Reisinger and others, 2015; Wollheim and others, 2017), but will likely not undergo substantial additional delays in delivery to coastal waters because surface transport is faster than groundwater transport. 

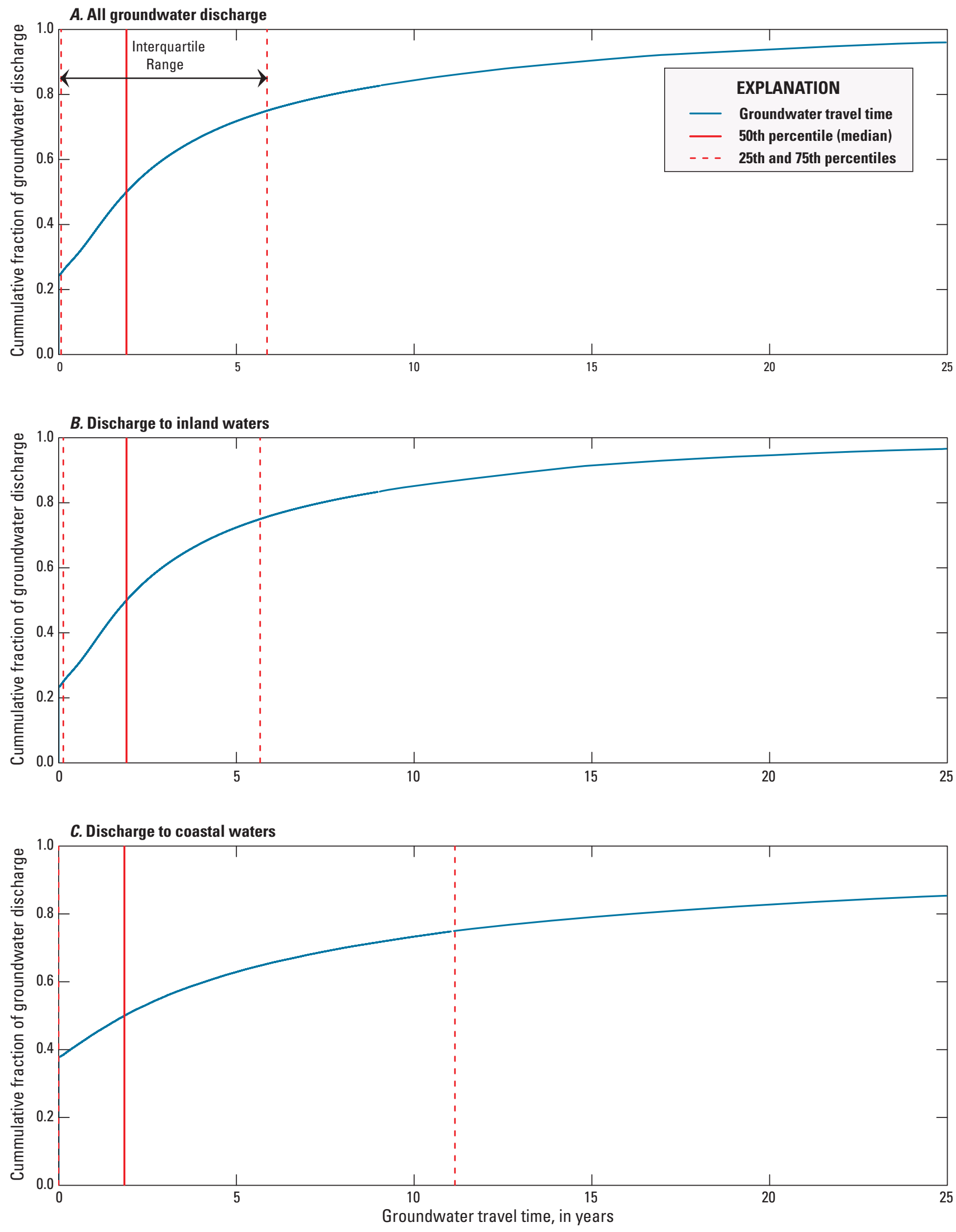

Figure 23. Cumulative fraction of groundwater discharge by travel time on the north shore of Long Island Sound (coastal Connecticut and adjacent areas of New York and Rhode Island) for groundwater discharge to $A$, all waters, $B$, inland waters, and $C$, coastal waters. 


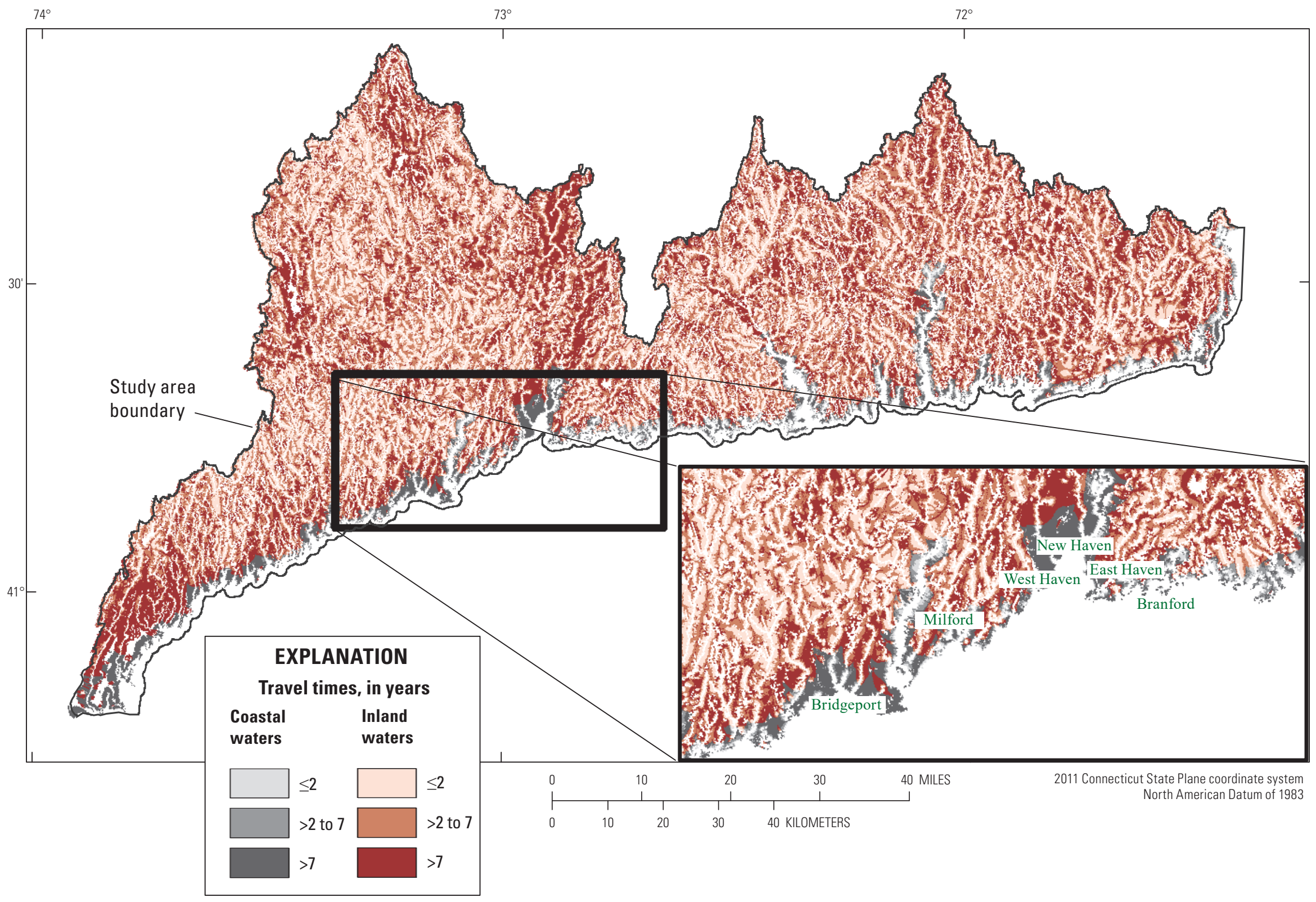

Figure 24. Groundwater travel times by discharge location (inland waters or coastal waters) on the north shore of Long Island Sound (coastal Connecticut and adjacent areas of New York and Rhode Island). Groundwater travel times are defined as the medians of the travel times from the point of recharge to the discharge location. Because transport times within the stream network are shorter than groundwater travel times, the mapped travel times are indicative of travel to coastal waters, regardless of the specific discharge location. 


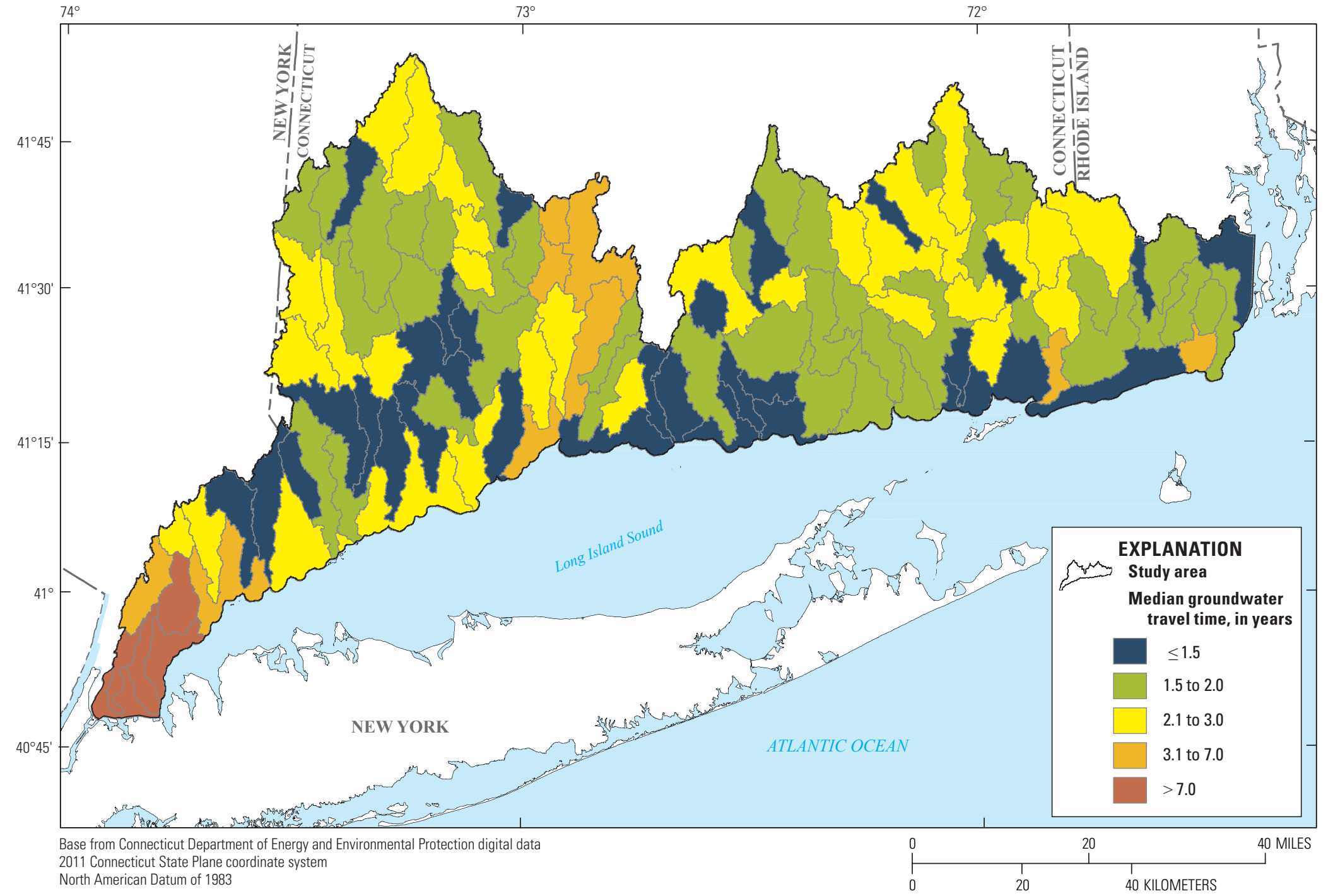

Figure 25. Median groundwater travel-times on the north shore of Long Island Sound (coastal Connecticut and adjacent areas of New York and Rhode Island) by extended HUC12 watershed. 


\section{Limitations and Factors Affecting Model Simulations}

Groundwater models improve understanding of groundwater-flow systems and provide predictive capabilities that are otherwise difficult to obtain; however, groundwater models are greatly simplified representations of the modeled hydrologic system. Simplifications are necessary because of limited data availability and computer resources, but they influence model outputs and predictions. In particular, simplifications and estimations related to temporal dynamics, water use, aquifer properties, and grid resolution may affect estimated travel times and groundwater budgets. Understanding the effects of simplifications and estimations in the modeldevelopment process is important for understanding and using the model predictions.

\section{Temporal Dynamics}

The model developed for this study is a steady-state model, meaning that it represents long-term average conditions. Steady-state models are robust for calculating long-term average groundwater budgets and for estimating long-term average travel times. Steady-state models, however, are not able to simulate interannual variation, seasonal patterns, or event dynamics. In this study, the simulated travel times are relatively short, with 38 percent of groundwater discharging within 1 year of recharge. Travel times of less than one year indicate that interannual and seasonal flow patterns may be important for understanding nutrient- and contaminanttransport patterns; however, assessing these temporal patterns was beyond the scope of this study.

\section{Water-Use Estimates}

Detailed water-use data were not available for the entire study area. Estimates were made regarding the locations and volumes of private-well withdrawals, septic return flows, and smaller public-supply wells. In addition, other types of wells, such as industrial and agricultural wells, were not included in the model. The effects of errors in the estimates of pumpage for private wells and septic return flows are likely small and localized because of their relatively small contribution to the overall water budget, and because private wells and septic systems in many areas co-occur, and the net withdrawals are small (fig. 10C). The effects of not including pumping from agricultural wells also are likely minor because agricultural wells account for approximately 0.5 percent of total groundwater outflows on the basis of county-level estimates of groundwater withdrawals and total groundwater outflows calculated in this study (Dieter and others, 2018). Omitting industrial wells may have a greater influence on the overall water budget because industrial withdrawals are estimated to compose a slightly larger fraction of total groundwater outflow (0.8 percent) (Dieter and others, 2018), but data on industrial withdrawals are not available.

\section{Applied- and Simulated-Pumping Rates}

The simulated withdrawal rates for some wells (136 of 80,800 wells) were reduced to prevent drying of the model cell. In total, these reductions reduced total well withdrawals by 22 percent and accounted for less than 1 percent of the water budget. Reduced pumping was more likely to be required (1) for public water-supply wells (14 percent) compared to private wells (less than 0.1 percent), (2) for higher flux wells (median non-reduced flux of $1.5 \mathrm{~m}^{3} / \mathrm{d}(370 \mathrm{gal} / \mathrm{d}$ ) for wells with reduced pumping compared to a median flux of $1.0 \mathrm{~m}^{3} / \mathrm{d}(260 \mathrm{gal} / \mathrm{d})$ for wells without reduced pumping), (3) in locations with shallow bedrock (median surficialmaterial thickness of $3.0 \mathrm{~m}$ for wells with reduced pumping compared to $4.6 \mathrm{~m}$ for wells without reduced pumping), (4) at higher altitudes (median altitude of $159 \mathrm{~m}$ for wells with reduced pumping compared to $120 \mathrm{~m}$ for wells without reduced pumping), and (5) closer to wetlands (median distance to a wetland of $63 \mathrm{~m}$ for wells with reduced pumping as compared to $104 \mathrm{~m}$ for wells without reduced pumping). Medians noted above were different at the 95-percent confidence level. Many public water-supply wells are located near streams or wetlands, where induced infiltration from surface water might maintain well yields (Kazmann, 1948). Infiltration of surface water is not simulated within the groundwater-flow model, which may be the reason why many public water-supply wells were simulated with reduced pumping. In addition, yields from some public water-supply wells with reduced pumping may be supported by localized deposits of higher conductivity sediments that are not represented in the regional-scale model. Finally, due to limited data on public water-supply withdrawals and the model spatial resolution, some withdrawals that are spread across multiple wells or well fields were simulated as single wells with aggregated withdrawal rates that lead to localized drying of the aquifer.

The effects of simulated reduced pumping on groundwater budgets and simulated travel times are minimal for most watersheds because the total reduction is a small fraction of the total simulated groundwater outflow (less than 0.001 percent to 9.9 percent, with a median less than 0.001 percent in extended HUC12 watersheds with reduced pumping) and any induced infiltration would be cycled locally. The reduction in pumping accounts for more than five percent of the total groundwater outflows in five extended HUC12 watersheds, all of which have near-stream (less than 150 meters from the nearest water body) public watersupply wells with high applied pumping rates (Rippowam River, map no. 11; 4,500 m³/d [1.2 Mgal/d]; Little River, map no. 39; 2,800 m³/d [0.74 Mgal/d]; Mill River, map no. 53; $18,000 \mathrm{~m}^{3} / \mathrm{d}$ [4.8 Mgal/d]; Mill Creek-Connecticut River, map no. $64 ; 12,000 \mathrm{~m}^{3} / \mathrm{d}[3.1 \mathrm{Mgal} / \mathrm{d}]$; and the Lower Pawcatuck River map no. $98 ; 6,500 \mathrm{~m}^{3} / \mathrm{d}$ [1.7 Mgal/d]) (fig. 2, table 1) 
that likely induced infiltration of surface water, which is not simulated in this model. In these watersheds, refinement of information about well-withdrawals, surficial materials, and stream-aquifer exchange processes may be needed. For all watersheds, reduced pumping would likely influence groundwater flowpaths and travel times in the vicinity of the wells with reduced pumping.

\section{Urban Water Dynamics}

In highly developed urban areas, humans have altered water budgets in complex ways. Storm and sanitary sewers can provide an additional inflow or outflow of water to or from the groundwater system (Lerner, 1990; Lerner, 2002; Wittenberg and Aksoy, 2010). Impervious cover can reduce (Kauffman and others, 2009) and change the spatial patterns of recharge (Han and others, 2017), which may lower groundwater tables. Disturbance associated with urbanization can change the physical properties of the subsurface (Hibbs and Sharp, 2012).

In this model, developed land cover is incorporated into the estimates of groundwater recharge, but other urban dynamics, such as sewers, were not simulated and may result in larger errors in urban areas. For example, areas of flooded terrestrial cells (fig. 18) are clustered around the highly developed areas in New York, and simulated travel times in these watersheds are outliers within the broader study area. Together, these data indicate that urban-water dynamics may be less well represented in this regional model, and further detailed studies may be needed to reliably estimate budgets and travel times in these areas.

\section{Hydrogeologic Framework}

Aquifer properties such as hydraulic conductivity, porosity, and depth to bedrock can vary, sometimes substantially, on the scale of tens of meters, but aquifer-property information is not available at fine spatial resolutions across the study area. In addition, the available data must be aggregated to the grid-cell resolution. Lack of data and aggregation to the grid cell results in the loss of local-scale heterogeneity in simulated aquifer properties. The simplified representation of the aquifer can be expected to reflect the broad spatial patterns of groundwater flow, but will likely not represent flow and discharge patterns caused by local features such as bedrock outcrops, small deposits of coarse or fine materials, or abrupt changes in the land-surface slope.

\section{Hydraulic Conductivity}

Hydraulic conductivity is a measure of how easily water flows through a material. In the model, hydraulic-conductivity values are calibrated for broad geologic-material groups. Hydraulic-conductivity values vary due to compression from the overburden (for unconsolidated sediments), fracture patterns (in fractured bedrock), and variations in sediment composition, among other causes. This heterogeneity in hydraulic-conductivity values for materials of the same type was not simulated in this model because of limited calibration data. Model areas where the actual hydraulic-conductivity values differ substantially from the calibrated values are more likely to be located where calibration data is particularly limited, and geologic properties and histories differ from those in the rest of the modeled area.

\section{Aquifer Porosity}

Aquifer porosity is inversely proportional to groundwater velocity and travel time. In this study, porosity values were estimated on the basis of geology. Like hydraulic conductivity, porosity values are heterogeneous, even within the same general type of material (Anderson, 1997). To quantify the effects of porosity on simulated groundwater travel times, the porosity across the study area was increased and decreased by 50 percent and the travel times were recomputed. The porosity of areas mapped as "open water" was not modified in the sensitivity analysis. Travel times were computed on the basis of simulated volumetric water flux per unit area of aquifer material (an output from the groundwater model) and the userspecified porosity. Varying the porosity did not change the distribution of groundwater discharge among coastal waters, inland waters, and wells (fig. 26). Increasing and decreasing the porosity, however, did result in a linear, inversely proportional change in the flow velocity and a linear, directly proportional change in the travel times due to the same volume of water moving through a larger (with increased porosity) or smaller (with decreased porosity) space.

\section{Depth to Bedrock}

The thickness of unconsolidated sediments, which is synonymous with the depth to bedrock, was determined from generalized maps that may not represent local heterogeneity in sediment thickness. At the extended HUC12 watershed level, mean unconsolidated-sediment thickness across the model cells was positively correlated with the median of the groundwater travel time calculated in this study (Spearman's rho $=0.81, p \leq 0.01)$. This suggests that groundwater travel times may be longer than simulated in areas with thicker-thanmapped unconsolidated sediments and shorter than simulated in areas with thinner-than-mapped unconsolidated sediments.

\section{Grid Resolution}

Model-cell resolution, which is frequently chosen on the basis of computational resources, may influence simulated travel times because it determines the level of detail at which geologic and hydrologic features are simulated in the model. In general, as the model-cell size increases, the required computational resources decrease, but some features will 

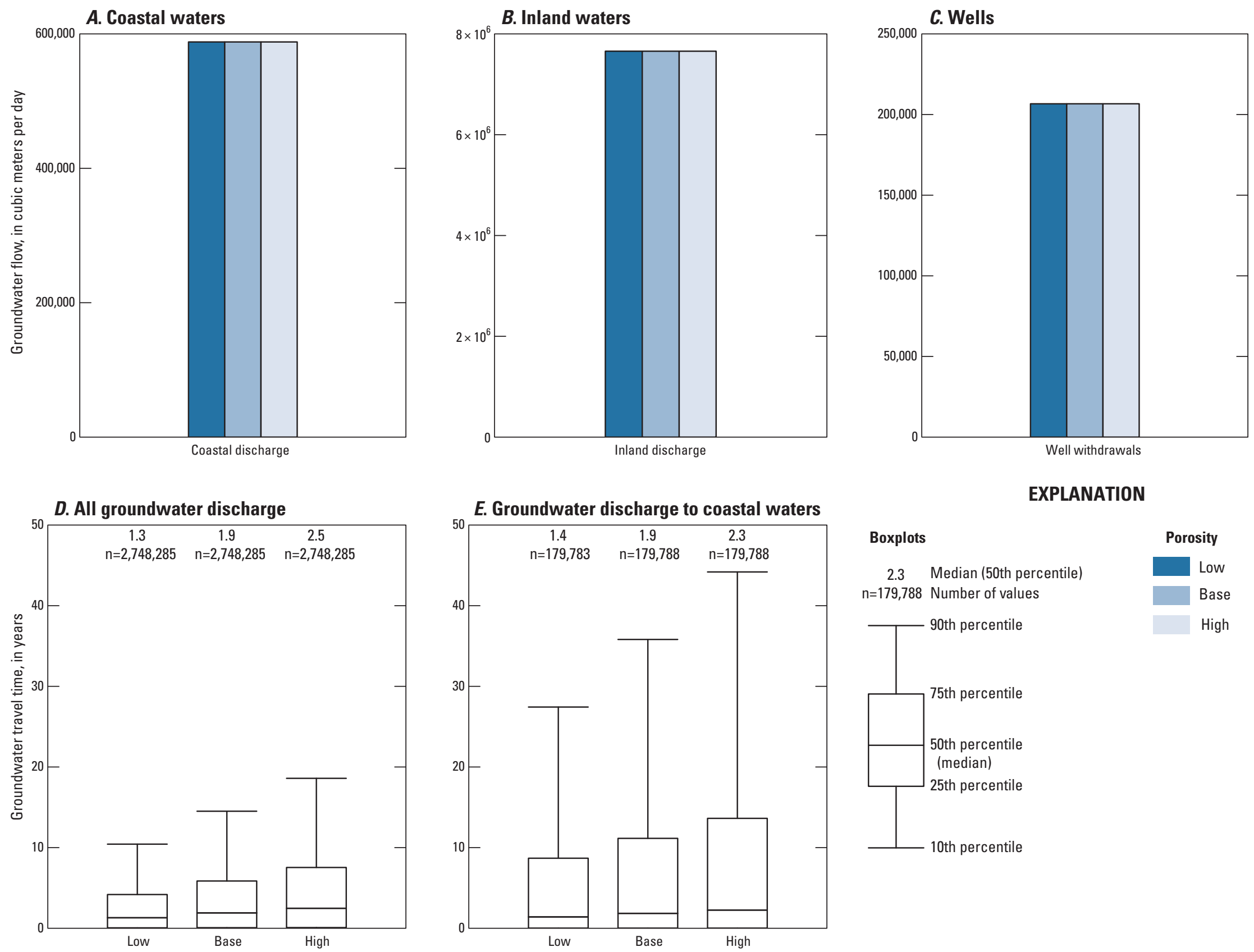

EXPLANATION

Boxplots

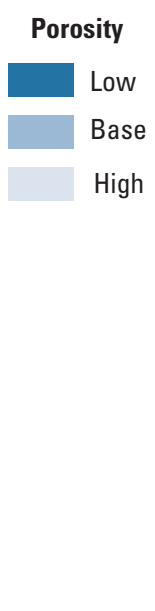

Figure 26. The effects of varying aquifer porosity on groundwater outflows to $A$, coastal waters, $B$, inland waters, and $C$, wells; and on $D$, travel times for all groundwater discharges, and $E$, groundwater discharge to coastal waters, north shore of Long Island Sound (coastal Connecticut and adjacent areas of New York and Rhode Island). 
be over-emphasized, and other features may not be represented or may be overly generalized. For example, an area of groundwater discharge might be simulated as one large cell rather than one small cell, or conversely, a localized deposit of coarse sediments may not be represented. To quantify the effects of model-grid resolution on the simulated groundwater travel times, three inset models of the Niantic River watershed (fig. 27) were developed with varying grid resolutions (304.8 $\mathrm{m}[1,000 \mathrm{ft}], 152.4 \mathrm{~m}[500 \mathrm{ft}]$, and $76.2 \mathrm{~m}$ [250 ft]). The inset models were developed in the same way as the primary groundwater model, except that the inset models were not calibrated due to the lack of calibration data available within the inset model area. The calibrated parameter values from the primary groundwater model were used for all three inset models.

Decreasing the cell size from $304.8 \mathrm{~m}(1,000 \mathrm{ft})$ to $152.4 \mathrm{~m}(500 \mathrm{ft})$ and $71.2 \mathrm{~m}(250 \mathrm{ft})$ resulted in a decrease in the volume of groundwater discharging to coastal waters (fig. 28). Decreasing the cell size resulted in an increase in the travel time for all groundwater discharge from a median of 0 years for the $304.8-\mathrm{m}(1,000-\mathrm{ft})$ model to 1.7 years for the $152.4-\mathrm{m}(500-\mathrm{ft})$ model and 1.8 years for the $71.2-\mathrm{m}(250-\mathrm{ft})$ model (fig. 28D). The effects of decreased cell size on travel times were more pronounced for discharge to coastal cells, in which travel times increased from 0 years for the $304.8-\mathrm{m}$ $(1,000-\mathrm{ft})$ model, to 2.8 years for the $152.4-\mathrm{m}(500-\mathrm{ft})$ model, and to 5.4 years for the $71.2-\mathrm{m}(250-\mathrm{ft})$ model. The increase in travel times with the finer resolution grid is largely due to mapping the groundwater discharge areas at a finer resolution. Any particle that begins in a model cell with groundwater discharge will have a travel time of 0 years, even if the groundwater is discharged over a relatively small fraction of the model-cell area. Reducing the grid resolution results in fewer model cells with groundwater discharge and therefore fewer particles that have a travel time of 0 (15 percent for the $71.2-\mathrm{m}$ [250-ft] grid cells, versus 30 percent and 53 percent for the 152.4-m [500-ft] and 304.8-m [1,000-ft] cells, respectively). Decreasing the grid resolution may result in more accurate estimates of travel time; however, it also increases the necessary computing resources and run times. In this study, the final 152.4-m (500-ft) model, rather than the final 304.8-m (1,000-ft) model, was used to improve the estimates of groundwater travel times without substantially increasing computational complexity. 


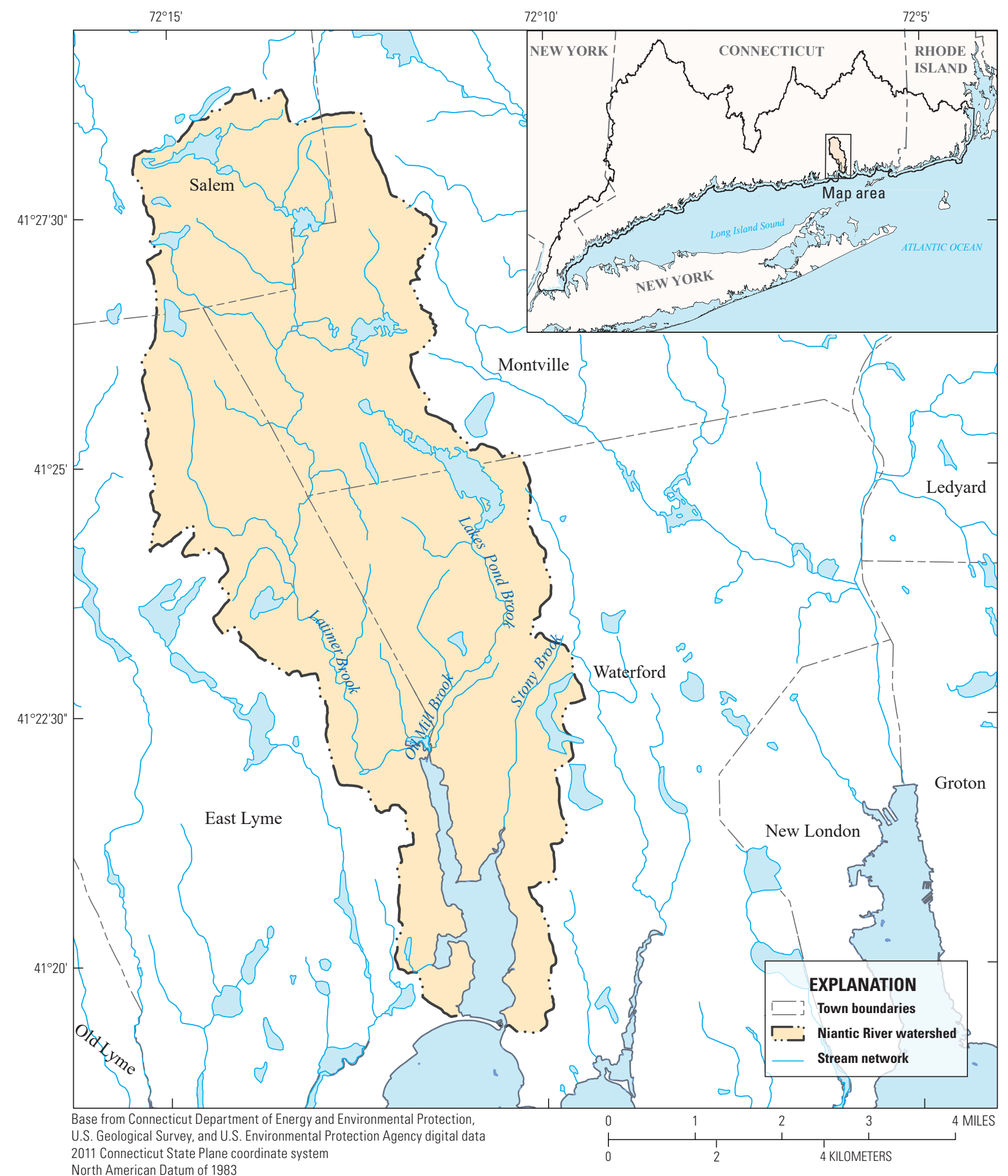

Figure 27. The Niantic River watershed, Connecticut. 

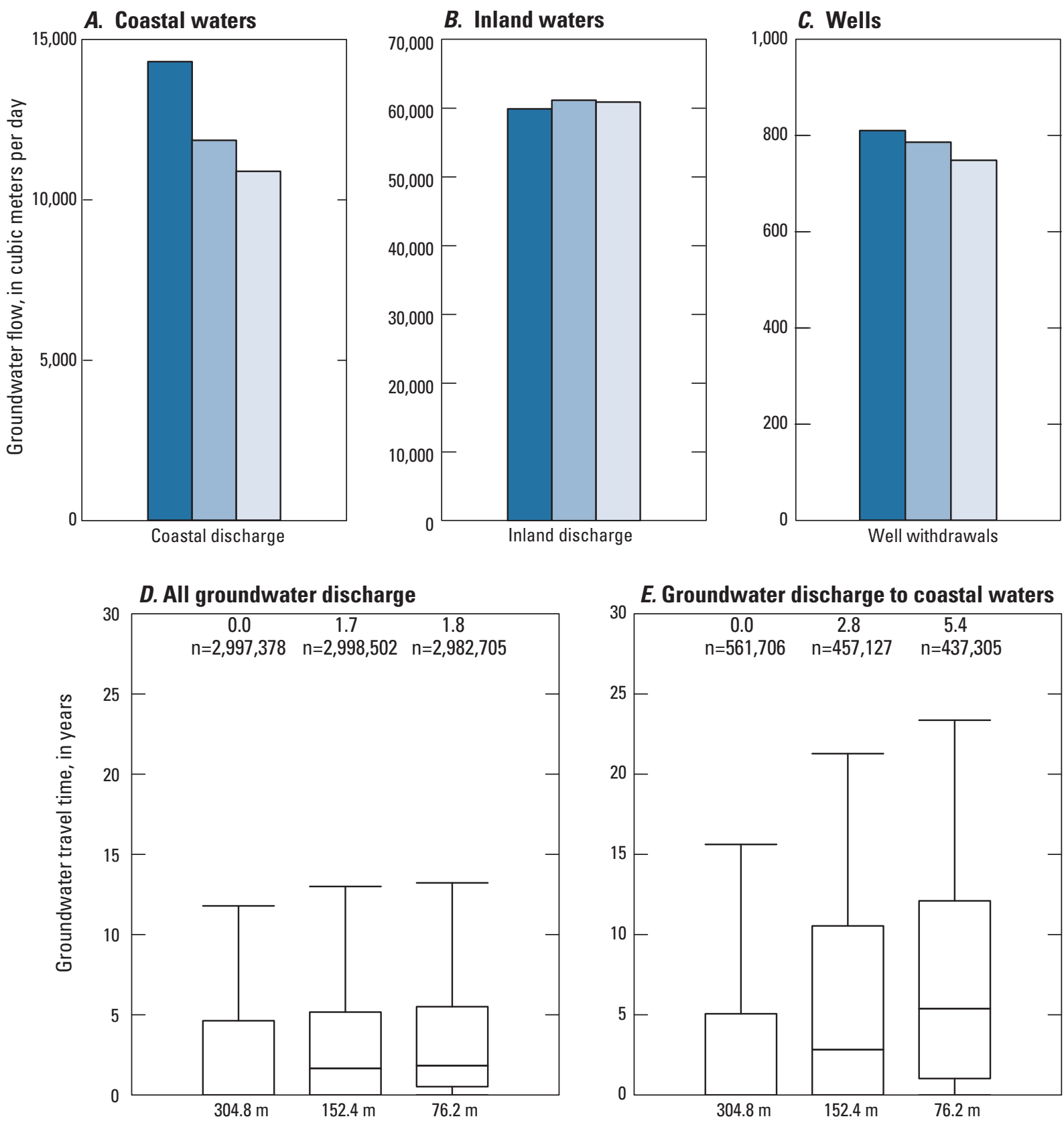

E. Groundwater discharge to coastal waters

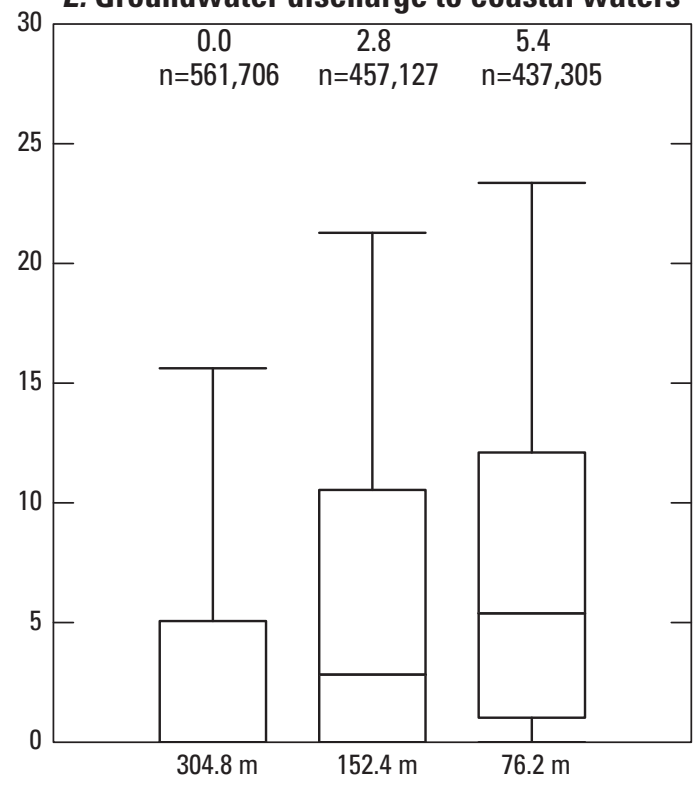

EXPLANATION

Boxplots

Grid resolution, in meters (m)

5.4 Median (50th percentile)

$\square 04.8$

$\mathrm{n}=437,305$ Number of values

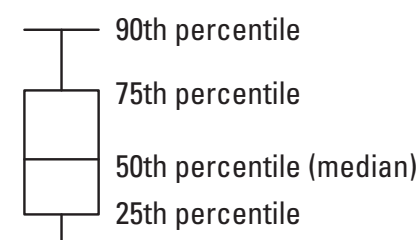

Figure 28. Groundwater outflows to $A$, coastal waters, $B$, inland waters, and $C$, wells with different grid-resolution values; $D$, groundwater travel times for all groundwater discharge, and $E$, groundwater discharge to coastal waters with different grid-resolution values, Niantic River watershed, Connecticut. 


\section{Simulation of Nitrogen Transport by Groundwater Within the Niantic River Watershed}

Groundwater nitrogen loads and travel times differ substantially across the landscape, with some areas contributing high loads with longer legacies and other areas contributing smaller loads. Understanding the spatial and temporal patterns of nitrogen loading, discharge, and attenuation from and in groundwater can inform management actions. For example, targeting management efforts to areas with short travel times might result in the greatest reduction of nitrogen to coastal waters in the near term, whereas areas with long travel times may require prolonged investment and (or) expectations of a delayed response. Groundwater-flow models can be used to determine the spatial patterns of groundwater nitrogen loading and travel times to inland and coastal waters at the watershed scale.

To demonstrate the feasibility and benefits of simulating groundwater nitrogen loading and travel times, nitrogen transport and loading were analyzed by using the regional groundwater model described above. The demonstration project used a simple nitrogen model that incorporates rates of nitrogen loading based on land-use and rates of attenuation based on geologic zones (Vaudrey and others, 2016a, b). The nitrogen model was linked with the regional groundwater-flow model to connect nitrogen loads with groundwater travel times. In addition to highlighting one potential use of the regional groundwater model, this demonstration project provided preliminary data on groundwater transport of nitrogen within the demonstration watershed.

The watershed for the demonstration project is the Niantic River watershed (fig. 27), a coastal watershed in eastern Connecticut. The Niantic watershed was selected because (1) it is one of the priority embayments for Connecticut's Second Generation Nitrogen Strategy: Long Island Sound (Connecticut Department of Energy and Environmental Protection, 2019a), and (2) measurements of surface-water nitrogen concentrations during base-flow conditions, which can be compared to the simulated values, were made during a prior investigation (Mullaney, 2013). Within the Niantic River estuary, eelgrass beds, an indicator of desirable nitrogen loads and water quality (Latimer and Rego, 2010; Vaudrey, 2008; Vaudrey and others, 2010), experience substantial interannual variations. The presence of eelgrass beds, combined with high interannual variability, suggests an opportunity to improve conditions within the estuary through targeted management efforts. Understanding spatial patterns of groundwater nitrogen loading and attenuation within the watershed could guide these efforts.

\section{Prior Investigations}

The Niantic River watershed was the focus of two prior USGS studies. In 2013, a study was conducted to quantify total nitrogen concentrations and instantaneous nitrogen loads in tributaries of the Niantic River estuary (Mullaney, 2013). Instantaneous nitrogen loads measured under base-flow conditions during the 2013 study were used for comparison with simulated values from the current study. A second study was conducted to determine the effects of sewering on nitrogen loads on one peninsula in the Niantic River estuary (Mullaney, 2015).

Nitrogen transport within the Niantic River watershed was also included in work by Vaudrey and others (2016a) quantifying nitrogen loading to embayments around LIS. That study focused on watershed-scale estimates of nitrogen loads for each embayment. The current demonstration project incorporates data on nitrogen input and loading from Vaudrey and others (2016a, b) to investigate nitrogen transport through the groundwater system in the Niantic River watershed and to quantify spatial patterns in loading and attenuation.

\section{Nitrogen-Transport-Model Development}

The nitrogen-transport model calculated groundwater nitrogen loads by using nitrogen inputs estimated from land-cover and land-use data, nitrogen-removal rates based on subsurface zone and geologic material, and groundwater flowpaths as simulated with particle tracking. The nitrogen inputs and loading factors are largely based on those estimated by Valiela and others (1997) and modified by Vaudrey and others $(2016 \mathrm{a}, \mathrm{b})$. The primary changes to nitrogen inputs for this study include (1) the use of raster datasets of wet and dry atmospheric deposition of nitrogen, (2) the estimation of septic inputs on the basis of a population density dataset rather than census blocks, and (3) the partitioning of nitrogen inputs between surface runoff and groundwater pathways. These changes are discussed in the subsequent section "Nitrogen Inputs."

\section{Particle Tracking}

Particle tracking can be a useful tool for understanding the fate of nitrogen within a groundwater-flow system, as well as estimating subsurface travel times and contributing areas (Walter, 2008; Walter and others, 2019). As with the regional groundwater-flow model, MODPATH (Pollock, 2012) was used for the particle-tracking simulation in the Niantic River watershed nitrogen model. MODPATH simulates advective transport through the groundwater-flow system. Denitrification, the reactive removal of dissolved nitrogen, and biological uptake are simulated through attenuation factors that are applied after the particle-tracking simulation. Dispersion and adsorption are not simulated with particle tracking. Particle-tracking parameters were the same as those 
used for the regional groundwater model, except that the density of particles within each cell was higher in the nitrogentransport simulation because the total simulation area was smaller. After the particle-tracking simulation, nitrogen loading and attenuation factors were applied.

\section{Nitrogen Inputs}

The model of nitrogen transport by groundwater accounts for nitrogen from three sources: atmospheric deposition, agricultural and lawn fertilizer, and septic systems (table 12, fig. 29). Nitrogen inputs from each source were estimated at the model-cell level as described below. The model-cell inputs for each source then were divided by the number of particles released within the cell to calculate nitrogen loads from each source for each particle.

Data on wet and dry nitrogen deposition for 2006 from the National Atmospheric Deposition Program (National Atmospheric Deposition Program, 2018) were used to estimate atmospheric-deposition inputs. The wet-deposition data include nitrogen that is transported via surface runoff, which is outside of the model boundary, as well as that which is in groundwater recharge, which is inside the model boundary. It was assumed that wet deposition is divided between surface runoff and recharge like precipitation. Approximately 23 percent of precipitation contributes to surface runoff, based on analysis of streamflow at two streamgages near the study area (01127500, Yantic River at Yantic, Conn.; 01194500, East Branch Eightmile River near North Lyme, Conn.); therefore, the wet-deposition inputs were decreased by 23 percent to account for wet-deposition nitrogen that does not enter the soil or groundwater system. It was assumed that a lesser fraction of dry-deposition nitrogen than wet-deposition nitrogen was transported through surface runoff; therefore, the dry-deposition data were decreased by 11.5 percent, which is 50 percent of the reduction for wet deposition, to account for surface transport. Because groundwater typically discharges rather than recharges to areas with open water, both wet- and dry-deposition inputs were further reduced by the percentage of the model cell covered by water. Finally, it was assumed that the spatial distribution of wet deposition was the same as the spatial distribution of groundwater recharge.

Nitrogen inputs from fertilizer were estimated from landcover data (fig. 30) and typical application rates. Nitrogen inputs on agricultural land were estimated by using the fraction of land within each model cell classified as "81-Pasture/ Hay" or "82-Row Crops" in NLCD (Homer and others, 2015) and an annual application rate of 86 kilograms of nitrogen per hectare per year (kg-N/ha/yr) (Vaudrey and others, 2016b). Nitrogen inputs to turf grass were estimated by using the fraction of land within each model cell classified as "Turf and Grass" in the Long Island Sound land-cover dataset (Center for Land Use Education and Research [CLEAR], 2016) and an annual application rate of $67.6 \mathrm{~kg}-\mathrm{N} / \mathrm{ha} / \mathrm{yr}$ (Vaudrey and others, 2016b). The loading rate for turf grass was based on surveys of fertilizing practices on residential lawns, parks, and recreational fields (Guillard and Fitzpatrick, 2011). It was assumed that some fertilizer was removed through surface runoff; therefore, the fertilizer inputs were reduced by 11.5 percent, the same reduction as was applied to dry deposition.

Nitrogen inputs from residential septic systems were estimated on the basis of a map of public sewer service areas (CT DEEP, 2019b [updated 1998 data]), 2010 population density (Falcone, 2016), and per resident nitrogen inputs of 4.8 kilograms of nitrogen per year $(\mathrm{kg}-\mathrm{N} / \mathrm{yr}$ ) per person (Vaudrey and others, 2016b) (fig. 30). The Niantic River watershed contains two nonresidential, subsurface wastewater-disposal systems with permits for subsurface sewage disposal. Locations and estimated nitrogen loads for these systems were compiled by Lombardo Associates, Inc., as part of an inventory of onsite wastewater systems for coastal Connecticut (CT DEEP, written commun., 2020; Barclay and Mullaney 2021a). Nitrogen inputs from the nonresidential subsurface systems were added to the septic inputs for the appropriate model cells.

Table 12. Estimated rates of nitrogen loading, by nitrogen source and land cover, on the north shore of Long Island Sound (coastal Connecticut and adjacent areas of New York and Rhode Island).

[Atmspheric deposition loading rates are based on the National Atmospheric Deposition Program (National Atmospheric Deposition Program, 2018). Estimated rates of nitrogen loading from fertilizer and septic systems are modified slightly from Vaudrey and others (2016a, b). Nitrogen inputs and outflows are given in kilograms of nitrogen per square kilometer per year ( $\mathrm{kg}-\mathrm{N} / \mathrm{km}^{2} / \mathrm{yr}$ ) or kilograms of nitrogen per year per person ( $\left.\mathrm{kg}-\mathrm{N} / \mathrm{yr} / \mathrm{person}\right)$. Land covers except for turf/grass are based on the 2011 National Land Cover Database (Homer and others, 2015). Turf/grass was determined by using data from the Center for Land Use Education and Research (CLEAR) (2016)]

\begin{tabular}{llc}
\hline \multicolumn{1}{c}{ Nitrogen source } & \multicolumn{1}{c}{ Land cover } & \multicolumn{1}{c}{ Nitrogen loading rate } \\
\hline Atmospheric deposition & Open water & $0 \mathrm{~kg}-\mathrm{N} / \mathrm{km}^{2} / \mathrm{yr}$ \\
& All other land covers & 34 to $1,604 \mathrm{~kg}-\mathrm{N} / \mathrm{km}^{2} / \mathrm{yr}$ \\
& & $\left(\right.$ median $\left.=885 \mathrm{~kg}-\mathrm{N} / \mathrm{km}^{2} / \mathrm{yr}\right)$ \\
Fertilizer & Agricultural land & $7,611 \mathrm{~kg}-\mathrm{N} / \mathrm{km}^{2} / \mathrm{yr}$ \\
& Turf/grass & $5,983 \mathrm{~kg}-\mathrm{N} / \mathrm{km}^{2} / \mathrm{yr}$ \\
Septic systems & All land covers & $4.8 \mathrm{~kg}-\mathrm{N} / \mathrm{yr} / \mathrm{person}$ \\
\hline
\end{tabular}


A. All nitrogen inputs

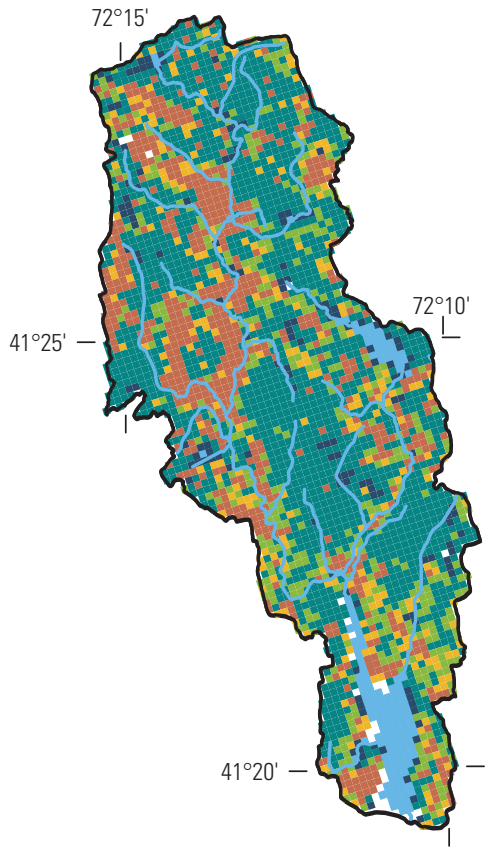

C. Fertilizer

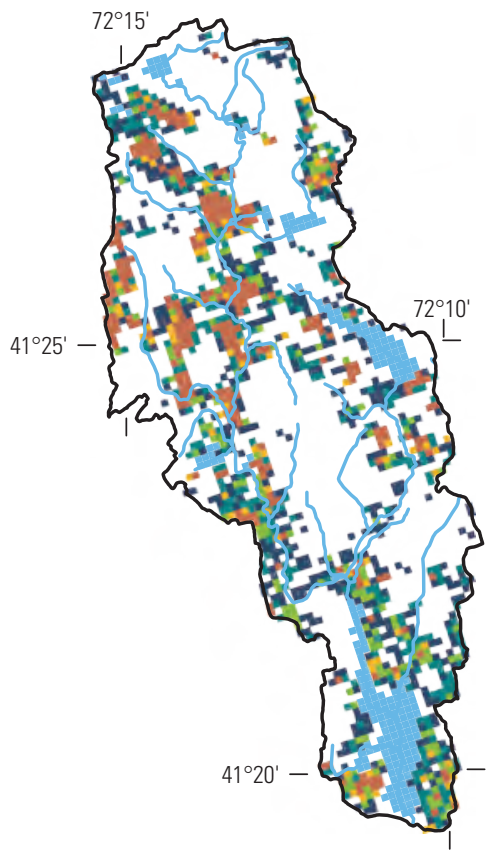

B. Atmospheric deposition

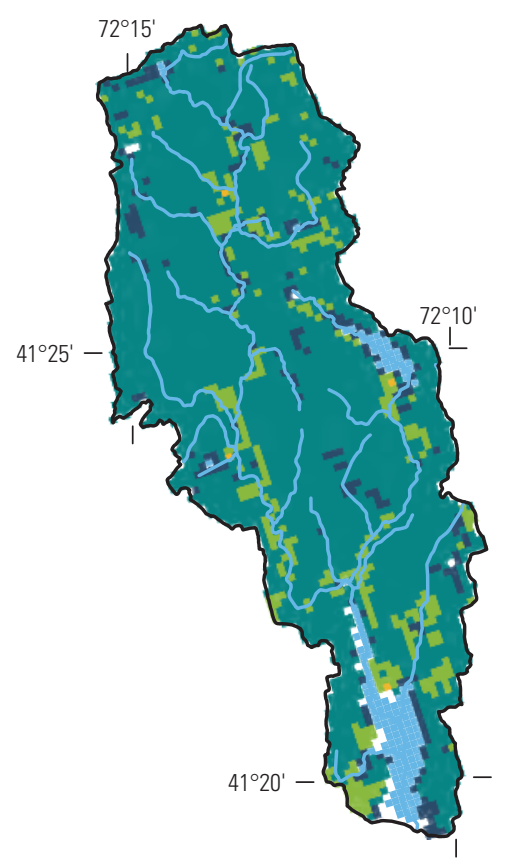

\section{EXPLANATION}

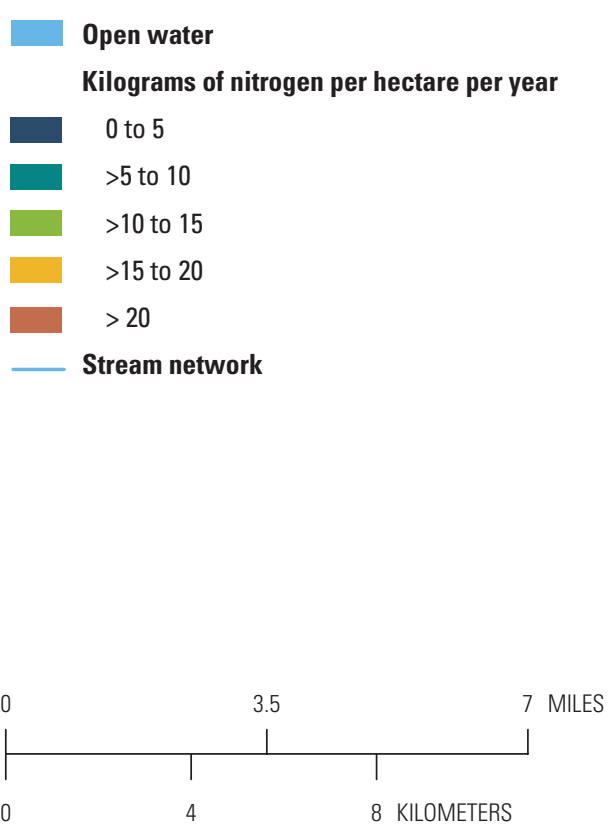

\section{Septic systems}

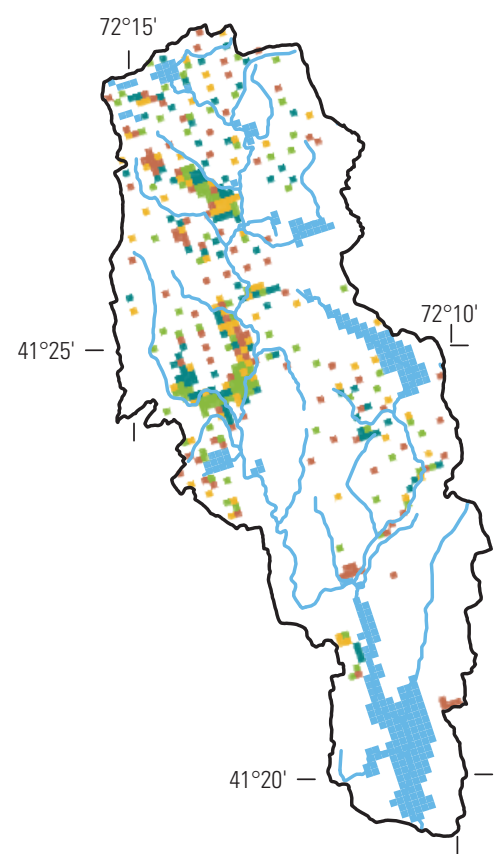

2011 Connecticut State Plane coordinate system North American Datum of 1983

Figure 29. Nitrogen inputs to the groundwater system in the Niantic River watershed, Connecticut. $A$, All nitrogen inputs. $B$, Atmospheric-deposition nitrogen inputs. $C$, Fertilizer nitrogen inputs. $D$, Septic-system nitrogen inputs. 
A. 2011 land cover

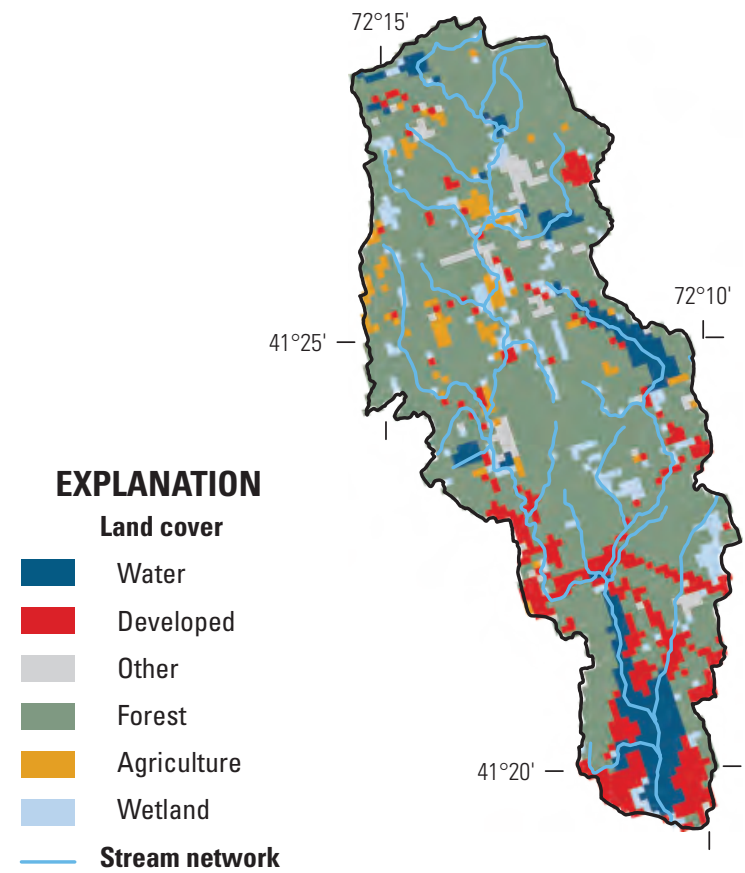

C. Percent grass and turf

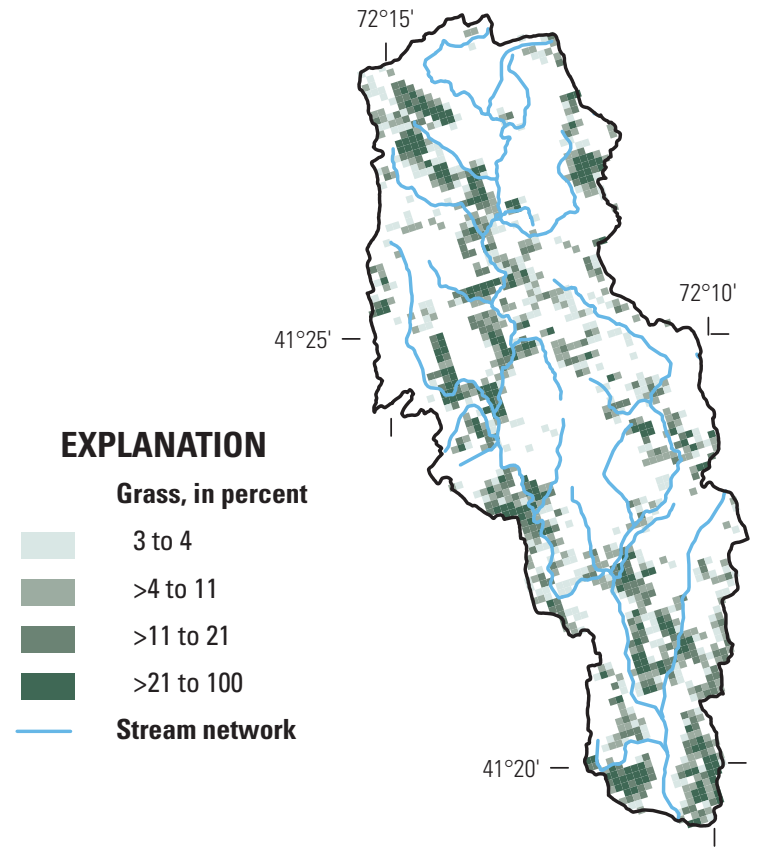

2011 Connecticut State Plane coordinate system North American Datum of 1983

\section{B. Percent agriculture}

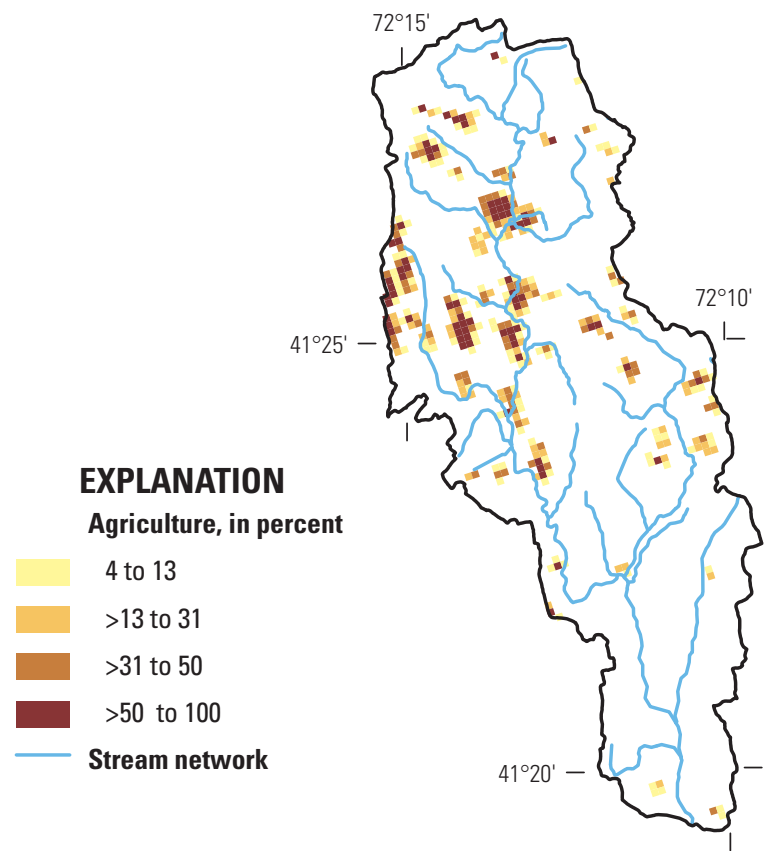

D. Population served by septic

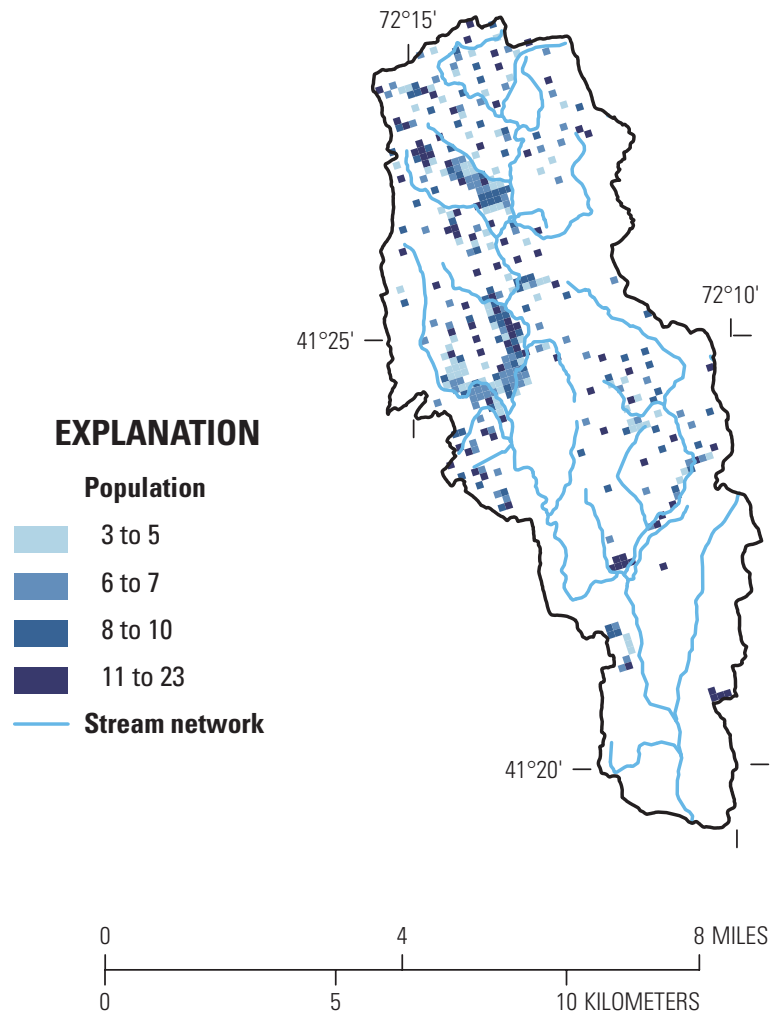

Figure 30. Land use in the Niantic River watershed, Connecticut. $A, 2011$ land cover. $B$, Percent agriculture. $C$, Percent turf and grass. D, Estimated population with septic systems. Land cover in 2011 and percent agriculture are from the 2011 National Land Cover Database (Homer and others, 2015). The percent turf and grass is from the 2010 Long Island Sound land-cover dataset (Center for Land Use Education and Research [CLEAR], 2016). The estimated population served by septic systems was estimated based on 2010 population density (Falcone, 2016) and sewer service areas as described in appendix 2. 


\section{Nitrogen Attenuation}

Nitrogen attenuation, or removal through biological uptake or reactive processes, was simulated by using removal fractions that were sequentially applied by subsurface zone (table 13, fig. 31). Removal fractions varied by nitrogen source (atmospheric deposition, fertilizer, or septic effluent), land cover, soil type, and subsurface zone.

For nitrogen from atmospheric-deposition and fertilizer sources, removal fractions were estimated for the soil zone, vadose zone, and aquifer or saturated zone on the basis of field measurements in the Waquoit Bay, Massachusetts, watershed (Valiela and others, 1997). For atmospheric-deposition sources, removal fractions in the soil zone varied by land cover, with rates ranging from 31 percent for high-intensity developed land to 75 percent for wetlands (Valiela and others, 1997; Vaudrey and others, 2016b). For fertilizer sources, Valiela and others (1997) estimated the soil-zone removal fraction to be 38 percent on the basis of lysimeter data. The removal fraction in the vadose zone below the soil zone was 61 percent for both atmospheric-deposition and fertilizer sources, except where the atmospheric deposition occurred over open water or wetlands, in which case the vadose-zone removal was zero percent. The removal fraction in the aquifer was 16 percent for nitrogen from both atmospheric-deposition and fertilizer sources (Vaudrey and others, 2016b), based on a literature review of aquifer nitrogen removal conducted by Jamie Vaudrey (Jamie Vaudrey, University of Connecticut, written commun., 2019).

For nitrogen from septic-system sources, removal fractions were estimated for the tank, leaching fields, plume (unsaturated zone below the leaching field), and aquifer. Nitrogen removal in the septic tank was estimated to be 5 percent on the basis of an analysis of nitrogen concentrations of waste entering and leaving septic-system tanks conducted by Valiela and others (1997). Removal fractions for the leaching fields and plume were the same values; they were estimated on the basis of soil texture and modeling work by Radcliffe and Bradshaw (2014), and they ranged from 1 percent for sand to 49 percent for sandy loam. Soil type was estimated on the basis of the Gridded Soil Survery (Soil Survey Staff, 2017a, b, c; U.S. Department of Agriculture, 2019). The

Table 13. Estimated rates of nitrogen attenuation, by nitrogen source, zone, and land cover, on the north shore of Long Island Sound (coastal Connecticut and adjacent areas of New York and Rhode Island).

[Estimated rates of nitrogen attenuation are modified from Vaudrey and others (2016a, b) and Radcliffe and Bradshaw (2014). Land covers are based on the 2011 National Land Cover Database (Homer and others, 2015). Soil types are based on the Gridded Soil Survey (Soil Survey Staff, 2017a, b, c; U.S. Department of Agriculture, 2019)]

\begin{tabular}{|c|c|c|c|}
\hline Nitrogen source & Zone & Land cover or soil type & $\begin{array}{l}\text { Nitrogen attenuation rate, } \\
\text { in percent }\end{array}$ \\
\hline \multirow{10}{*}{$\begin{array}{r}\text { Atmospheric } \\
\text { deposition }\end{array}$} & \multirow[t]{7}{*}{ Soil } & Open water & 55 \\
\hline & & Developed, medium intensity & 46 \\
\hline & & Developed, high intensity & 31 \\
\hline & & Wetlands & 75 \\
\hline & & $\begin{array}{l}\text { Planted/cultivated; pasture; developed, open space; } \\
\text { developed, low intensity }\end{array}$ & 62 \\
\hline & & Barren land & 32 \\
\hline & & Forest; shrubland; herbaceous & 65 \\
\hline & \multirow[t]{2}{*}{ Vadose zone } & Open water and wetlands & 0 \\
\hline & & All land cover except open water and wetlands & 61 \\
\hline & Aquifer & All land covers & 16 \\
\hline \multirow[t]{3}{*}{ Fertilizer } & Soil & All land covers & 38 \\
\hline & Vadose zone & All land covers & 61 \\
\hline & Aquifer & All land covers & 16 \\
\hline \multirow[t]{6}{*}{ Septic systems } & Septic tank & All land covers & 5 \\
\hline & \multirow{4}{*}{$\begin{array}{l}\text { Leaching field and } \\
\text { plume }\end{array}$} & Loamy sand & ${ }^{1} 10$ \\
\hline & & Sand & ${ }^{1} 1$ \\
\hline & & Silty loam & 136 \\
\hline & & Sandy loam & 149 \\
\hline & Aquifer & All land covers & 16 \\
\hline
\end{tabular}

${ }^{1}$ Percentage was applied twice: once for the leaching field and once for the plume. 
A. General nitrogen attenuation process

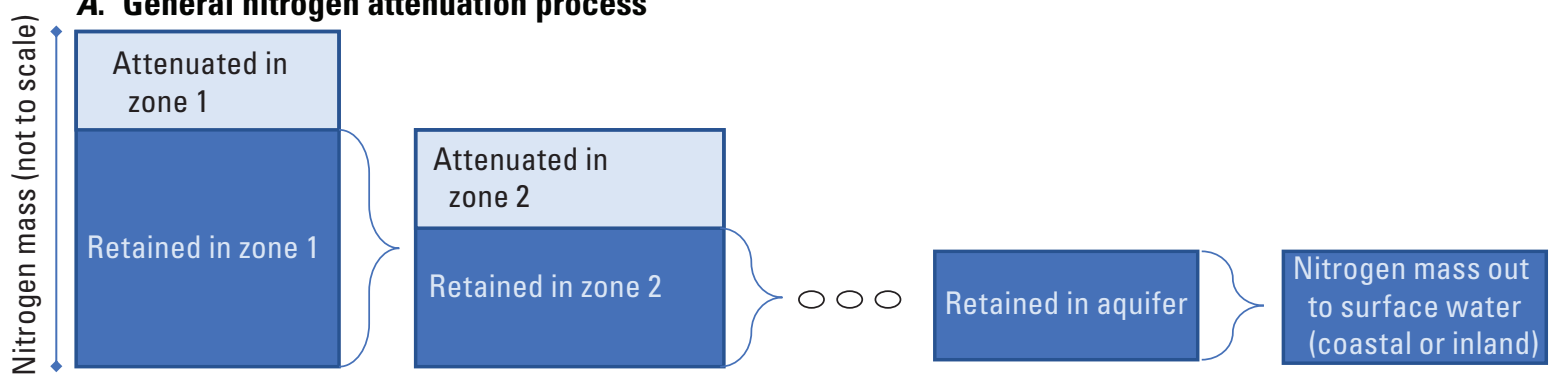

B. Example for a particle with fertilizer nitrogen inputs $(10.0 \mathrm{~g}-\mathrm{N})$ :

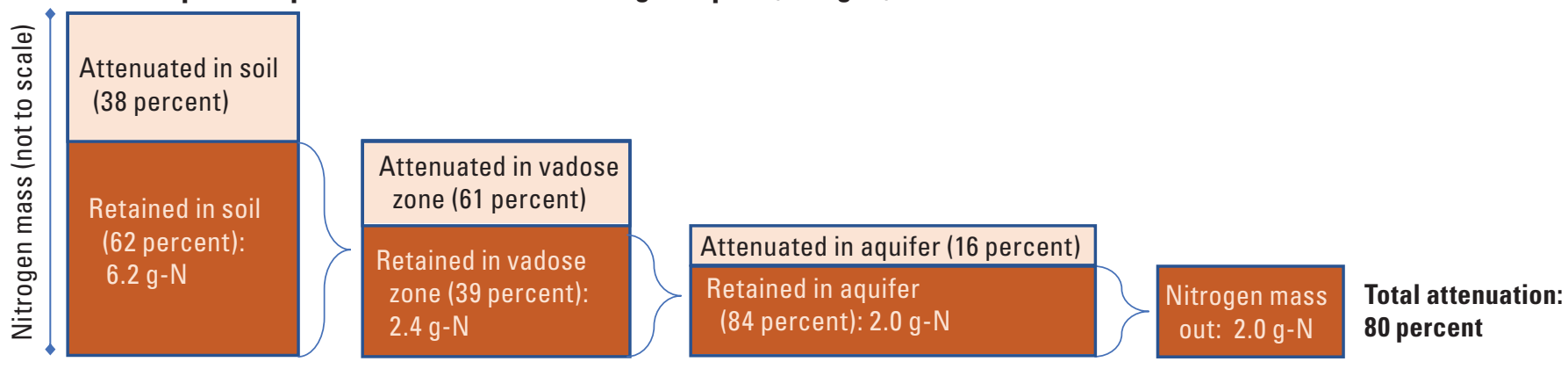

Figure 31. Schematics of the sequential nitrogen attenuation calculations shown $A$, in general, and $B$, with an example for a particle with nitrogen inputs from fertilizer. $\mathrm{g}-\mathrm{N}$, grams of nitrogen.

removal fractions for the leaching fields and the plume were applied twice, once for the leaching fields and again for the plume. The removal fraction in the aquifer was 16 percent, the same value used for aquifer removal of nitrogen from atmospheric-deposition and fertilizer sources (Vaudrey and others, 2016a, b).

\section{Nitrogen-Model Evaluation}

Simulated groundwater nitrogen loads were compared to instantaneous nitrogen loads observed during base-flow conditions in June 2005 (Mullaney, 2013). In June 2005, instantaneous loads were calculated for 11 subbasins within the watershed (fig. 32). In this study, observations from adjacent subbasins were combined to generate an additional 9 instantaneous loads, for a total of 20 observation basins. Simulated nitrogen loads were compiled for each observation basin by summing the attenuated mass of nitrogen discharging to surface waters within the basin. The simulated and observed loads are estimated by using different aspects of the hydrologic system - the observed loads are based on stream concentration and stream discharge, and the simulated loads are based on groundwater delivery to the stream - but comparing the estimates can yield information about the system and the simulation.

Simulated and observed nitrogen loads were compared for each observation basin (fig. 33). In general, the simulated loads were higher than the observed loads, with a mean error of 4.56 kilograms of nitrogen per day (10.1 pounds of nitrogen per day). The mean error is the average difference between the simulated and observed values. Some of the difference between the simulated and observed nitrogen loads likely is due to denitrification in sediments at the point of discharge (Billen and others, 2018) or instream processes, such as biological uptake and denitrification (Mulholland and others, 2008; Reisinger and others, 2015), that remove groundwatertransported nitrogen between the point of groundwater discharge and the subbasin outlet. In addition, some of the difference may be due to nitrogen removed through reservoir withdrawals. These processes were not simulated; therefore, the simulated loads would be expected to be higher than observed. In addition, the simulated loads reflect long-term average conditions, whereas the observed loads are a pointin-time snapshot. The observed flows were measured on a day with below-average stream discharge, which may have resulted in observed loads that were below the long-term average (Mullaney, 2013). Finally, precise rates and spatial patterns of nitrogen loading and attenuation are unknown, resulting in uncertainty in simulated values. Overall, the simulated and observed loads trend similarly, as indicated by the correspondence to the 1-to-1 line (fig. 33).

\section{Nitrogen-Transport-Model Results}

Based on this demonstration simulation and estimated rates of nitrogen input and attenuation, groundwater transports an estimated $22,000 \mathrm{~kg}-\mathrm{N} / \mathrm{yr}(2.9 \mathrm{~kg}-\mathrm{N} / \mathrm{ha} / \mathrm{yr})$ to streams, rivers, and coastal waters within the Niantic River watershed (fig. 34, table 14). This load represents an overall removal rate of 81 percent of input loads. Approximately 36 percent 


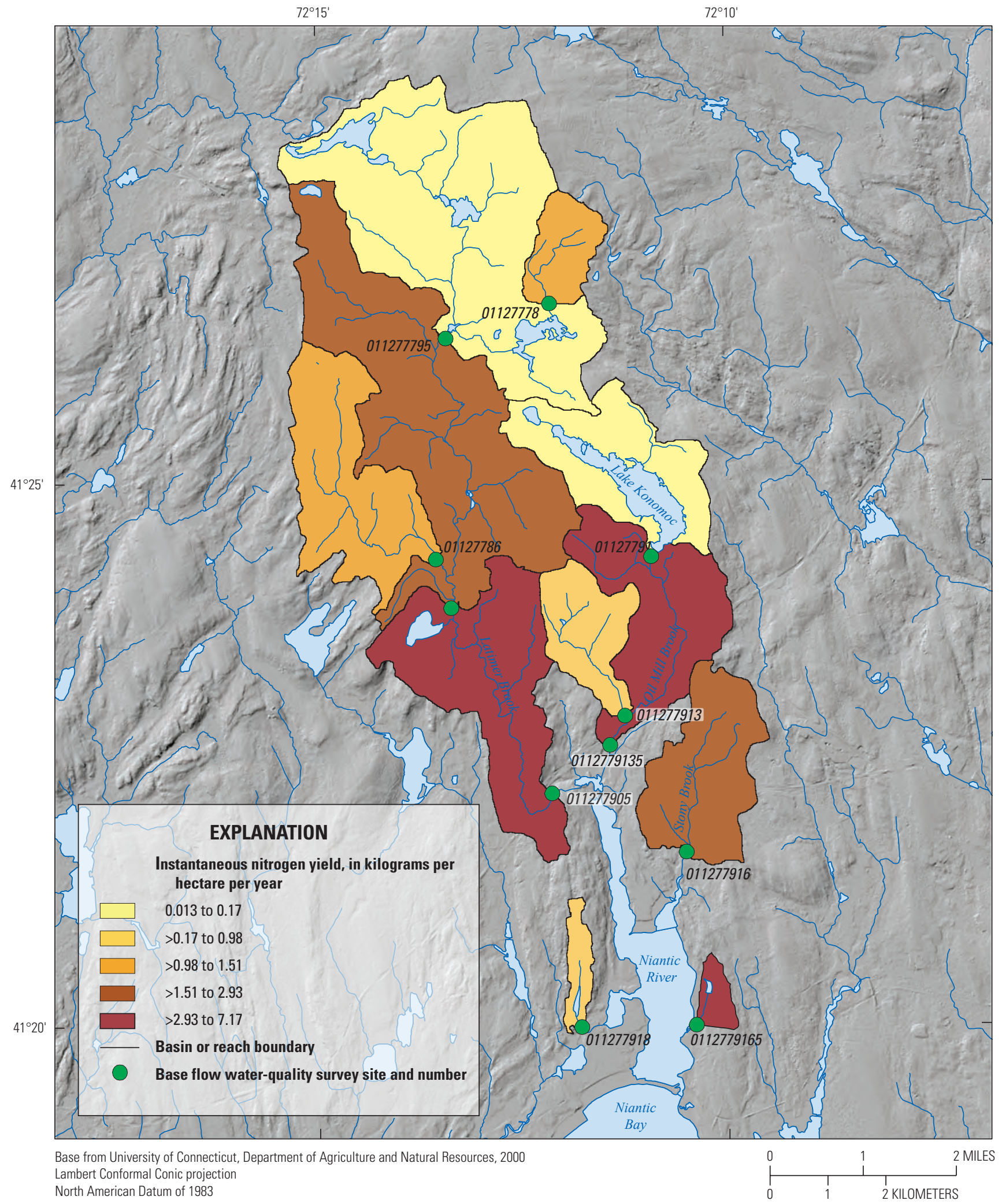

Figure 32. Nitrogen yields from selected basin segments in the Niantic River watershed, Connecticut, June 1-3, 2005. Figure modified from Mullaney (2013). 

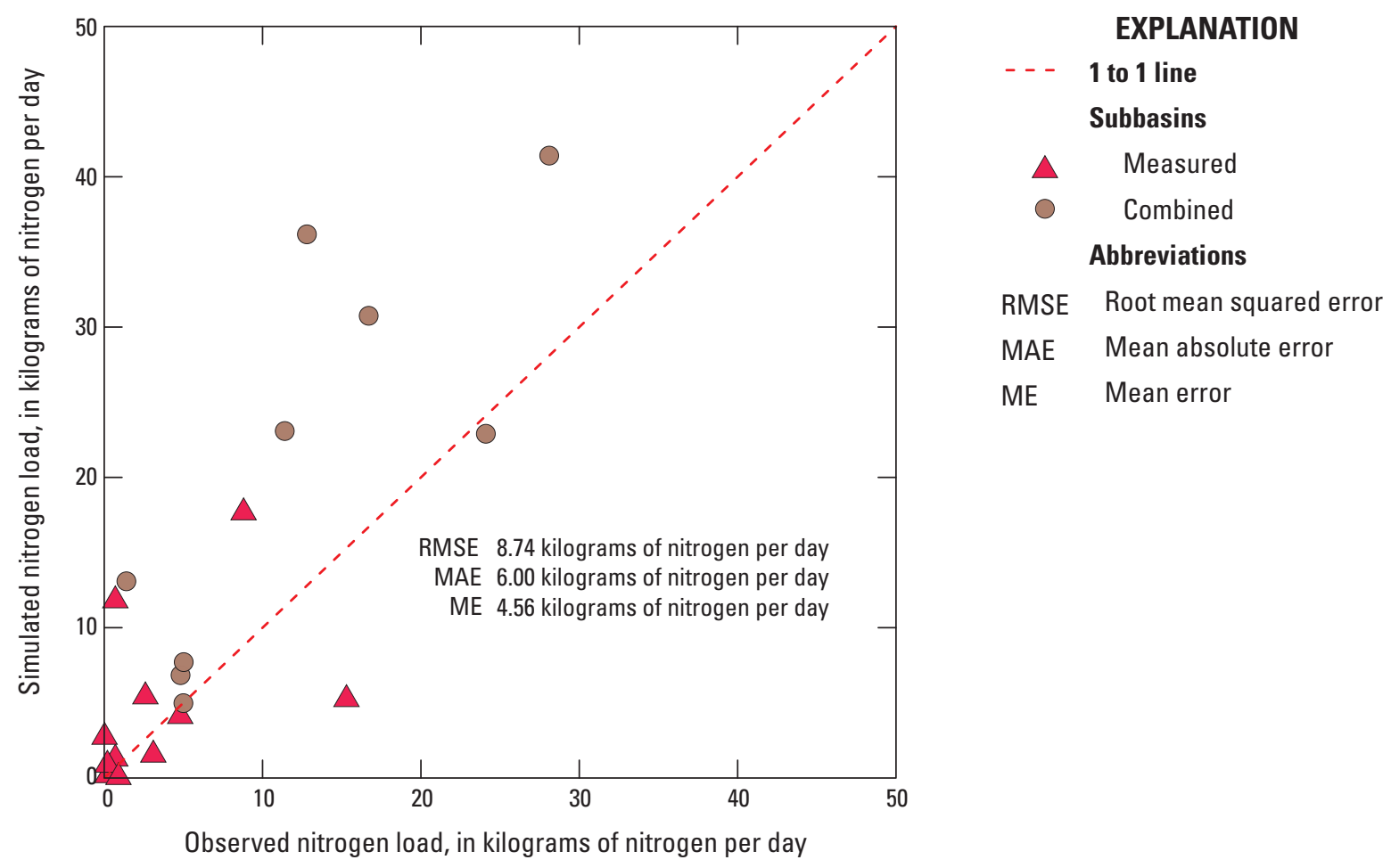

EXPLANATION

\begin{tabular}{ll} 
& Subbasins \\
& Measured \\
\multicolumn{1}{c}{ Abbreviations } \\
RMSE & Root mean squared error \\
MAE & Mean absolute error \\
ME & Mean error
\end{tabular}

Figure 33. Observed and simulated base-flow nitrogen load in the Niantic River watershed, Connecticut. Observed values are from Mullaney (2013).

of discharging nitrogen is from atmospheric-deposition sources, 38 percent from fertilizers, and 26 percent from septic systems. Combined removal rates varied by nitrogen source, with 87-percent removal of atmospheric-deposition nitrogen, 80-percent removal of fertilizer nitrogen, and 59-percent removal of septic-system nitrogen. Aggregate rates of loading and removal are a function of the loading- and removal-rate estimates used in the demonstration study and are independent of the hydrologic simulation. The groundwater-transport model determines the temporal discharge patterns and influences the spatial discharge patterns.

These simulation results suggest that most of the groundwater-transported nitrogen ( 88 percent) discharges to inland waters and that 12 percent discharges directly to coastal waters (fig. 35, table 14). Attenuation rates for groundwatertransported nitrogen and relative source contributions did not differ between nitrogen discharged to coastal water and nitrogen discharged to inland waters (81-percent removal). Septic systems contributed a greater fraction of nitrogen discharged to inland waters (27 percent) than of nitrogen discharged to coastal waters (20 percent), most likely because septic systems primarily are inland, away from coastal waters (fig. 29D). Atmospheric deposition and fertilizer contributed a slightly smaller fraction of nitrogen discharged to inland waters (36 percent for atmospheric deposition and 38 percent for fertilizer) compared to nitrogen discharged to coastal waters ( 38 percent for atmospheric deposition and 42 percent for fertilizer).

Simulated nitrogen loads from groundwater to surface water at the point of discharge, in both inland and coastal waters, ranged from 0 to more than $75 \mathrm{~kg}-\mathrm{N} / \mathrm{h}$ a of waterbody per year (fig. 36A), with a median of $12 \mathrm{~kg}-\mathrm{N} / \mathrm{ha}$ of waterbody per year. The rates of nitrogen loading to surface water were highest along the stream network in the northwestern portion of the watershed. Removal percentages ranged from 33 to 93 percent (fig. 36B), with a median of 85 percent.

Subsurface travel times for groundwater-transported nitrogen ranged from less than 1 day to more than 100 years, with a median of 1.6 years (fig. 37). Travel times for nitrogen discharged to inland waters were slightly shorter (median of 1.5 years) than for nitrogen discharged to coastal waters (median of 2.7 years). Nitrogen sources near streams tended to have shorter subsurface transport times, and sources with longer transport times tended to cluster on hillslopes and along watershed divides (fig. 38). In general, simulation results suggest the Niantic River watershed does not have spatially expansive areas with multidecadal nitrogen travel times; however, 5 percent of nitrogen has a travel time greater than 20 years, suggesting nitrogen legacies may be substantial in some isolated areas. 
Table 14. Simulated nitrogen inputs, outputs, and removal rates by discharge location and nitrogen source within the Niantic River watershed, Connecticut.

[Nitrogen inputs and outflows are given in kilograms of nitrogen per year]

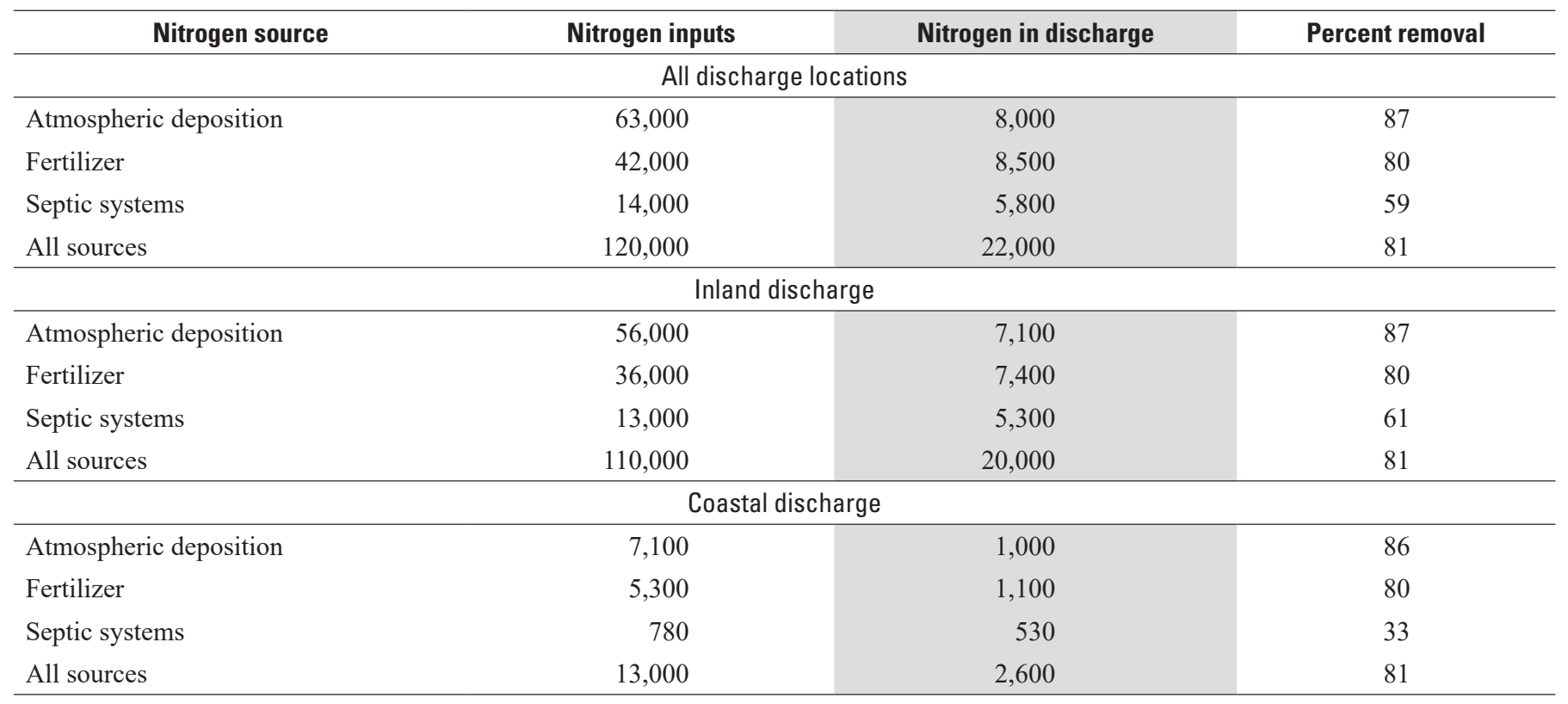

\section{Comparison with Nitrogen Loads from Other Studies}

Vaudrey and others (2016a, b) also calculated watershedwide nitrogen loads to the Niantic River, allowing for comparison between the studies. Although many of the rates of nitrogen loading and attenuation used here were the same as in Vaudrey and others (2016a, b), that study and the current study calculated different estimates of nitrogen loads because of differences in (1) included pathways (groundwater-transport pathways in this study as compared to all transport pathways in Vaudrey and others [2016a]), (2) simulated processes (this study did not simulate nitrogen removal in ponds, and Vaudrey and others [2016a, b] incorporated a 50-percent reduction in nitrogen from flow through ponds), and (3) analysis approach.

The watershed-wide nitrogen loads from septic systems $(5,800 \mathrm{~kg}-\mathrm{N} / \mathrm{yr})$ calculated as part of this study are slightly higher than those calculated by Vaudrey and others (2016a) $(4,600 \mathrm{~kg}-\mathrm{N} / \mathrm{yr})$. These differences are largely due to

1. differences in estimating the population served by septic systems (a population density raster was used in this study, with an estimate of 3,000 people served by septic systems, as compared to a census block-based analysis in Vaudrey and others [2016a, b], with an estimate of 3,600 people served by septic systems),

2. the inclusion in Vaudrey and others $(2016 a, b)$ of nitrogen removal in ponds, which is not simulated in this study, and
3. differences in soil-texture-based rates of septic nitrogen attenuation (a spatially uniform rate of 30 percent was used in Vaudrey and others [2016a, b], based on soil texture across the watershed, as compared to spatially heterogeneous rates with a mass-weighted mean of 32 percent in this study).

The watershed-wide loads of nitrogen from fertilizer in this study $(8,500 \mathrm{~kg}-\mathrm{N} / \mathrm{yr})$ were also higher than those calculated by Vaudrey and others $(2016 \mathrm{a}, \mathrm{b})(5,900 \mathrm{~kg}-\mathrm{N} / \mathrm{yr})$. This difference is due to (1) the inclusion in Vaudrey and others (2016a) of nitrogen removal in ponds, which is not simulated in this study, and (2) removal of fertilizer inputs transported by overland flow, which bypasses the groundwater system, in this study but not in Vaudrey and others (2016a).

The watershed-wide nitrogen loads from atmospheric deposition from this study $(8,000 \mathrm{~kg}-\mathrm{N} / \mathrm{yr})$ are also higher than the atmospheric-deposition loads from Vaudrey and others $(2016 \mathrm{a}, \mathrm{b})(7,500 \mathrm{~kg}-\mathrm{N} / \mathrm{yr})$. This difference is due to (1) the inclusion in Vaudrey and others (2016a) of nitrogen removal in ponds, which is not simulated in this study, and (2) removal of atmospheric-deposition inputs transported by overland flow, which bypasses the groundwater system, in this study but not in Vaudrey and others (2016a, b).

In a study focused on the effects of sewering on groundwater nitrogen, Mullaney (2015) estimated groundwater nitrogen loads of approximately 8,600 kg-N/yr (19,000 lb-N/yr) into the lower Niantic River watershed on the basis of measured groundwater nitrogen concentrations and estimated groundwater-flow rates. The estimate for that area from this study was 4,000 kg-N/yr. The difference in estimates is likely 


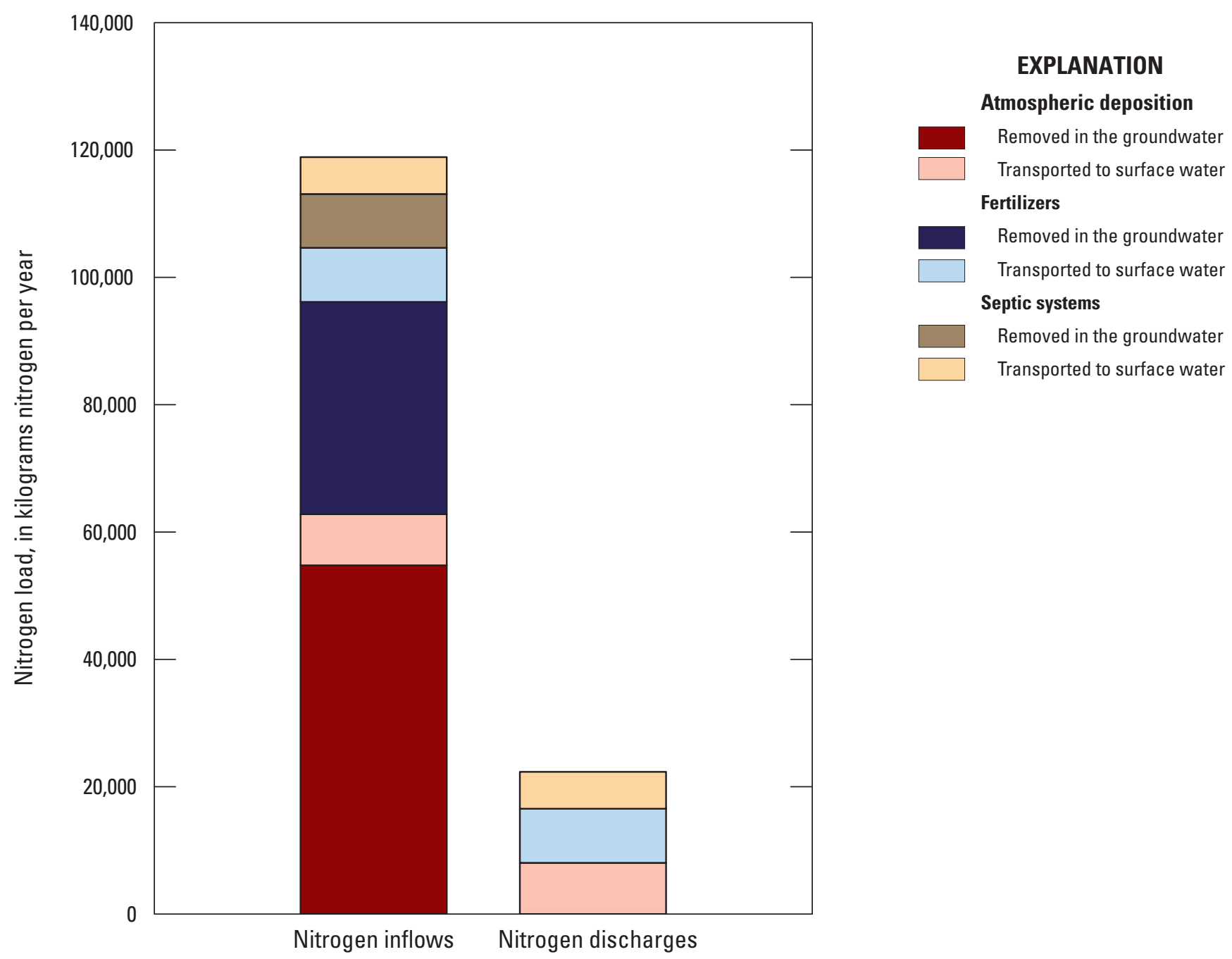

Figure 34. Nitrogen inputs and attenuation in the Niantic River watershed, Connecticut.

due to differing methods between the studies. Mullaney (2015) used observed nitrogen concentrations, estimated groundwater-flow rates, and the assumption, based on water chemistry data, that no further nitrogen would be removed prior to discharge. The current study used estimated nitrogen inputs and removal rates, as well as simulated groundwaterflow rates, to estimate annual groundwater nitrogen loads.

\section{Limitations}

Substantial uncertainty exists in the estimated nitrogen loading and attenuation factors and spatial patterns used in this study. Available data on rates of nitrogen loading and attenuation are sparse, and the existing data suggest rates are highly variable (Guillard and Fitzpatrick, 2011; New York State Department of Environmental Conservation, 2014; Stony Brook University Center for Survey Research, 2006; U.S. Environmental Protection Agency, 2002; Valiela and others, 1997). For this demonstration project, loading and attenuation factors, primarily based on those from Vaudrey and others (2016a, b), which were modified for conditions in coastal Connecticut, were adjusted as described in the section "Nitrogen-Transport-Model Development" and used. Other studies in nearby or similar areas have used different loading and attenuation factors (for example: Ator, 2019; Gold and others, 1990; Shimizu and others, 2018; Valiela and others, 1997). In particular, aquifer denitrification is a complex and variable process, with removal rates depending upon the flowpath length and the biogeochemical setting. Spatially varying rates of aquifer denitrification are not well constrained at the watershed scale; therefore, the rates used in this demonstration project represent a highly simplified estimate. Higher loading rates would result in greater nitrogen loads, and higher attenuation factors would result in smaller nitrogen loads. A thorough review of existing data on nitrogen loading and removal rates may yield more precise estimates of catchment scale loading and removal but is beyond the scope of the demonstration presented in this study. 

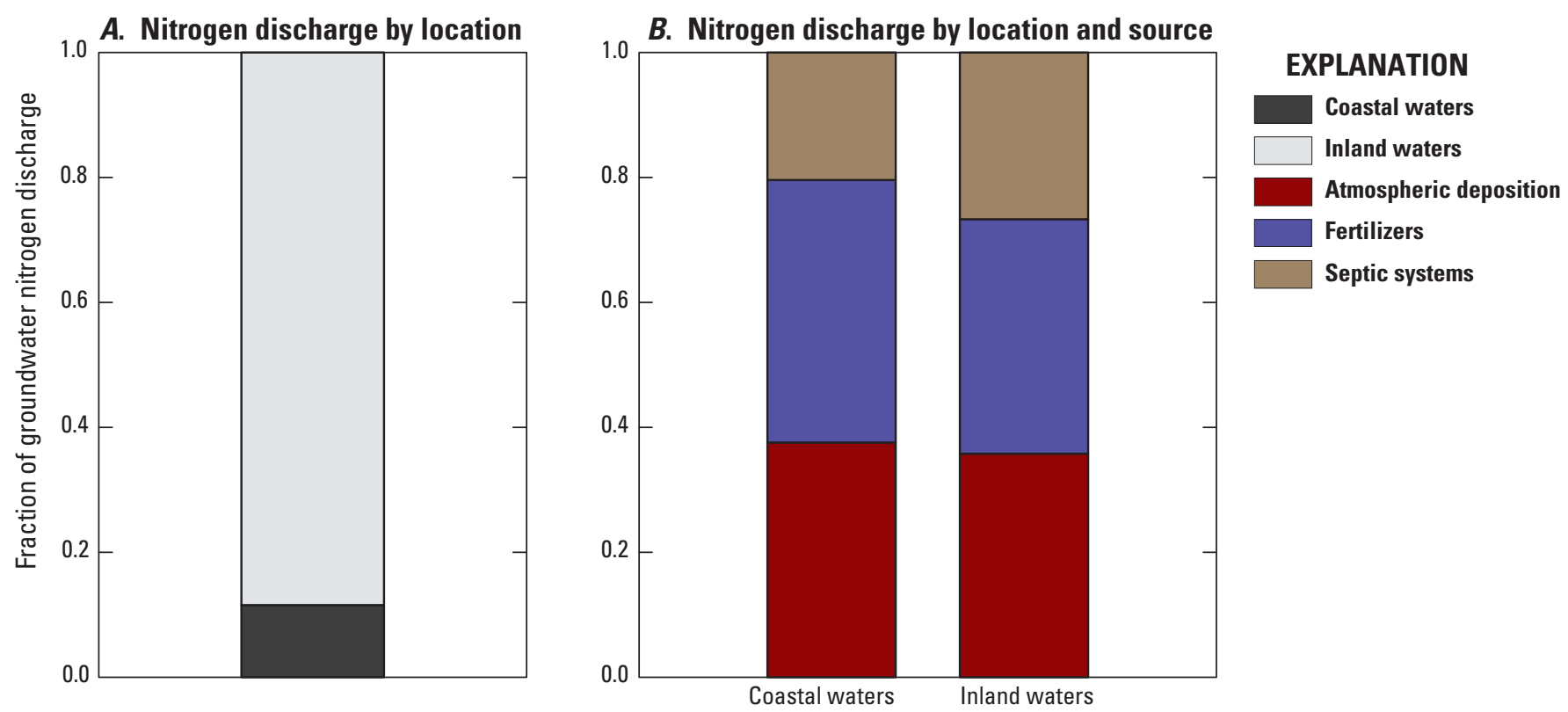

Figure 35. Fraction of groundwater nitrogen by discharge location and nitrogen source in the Niantic River watershed, Connecticut. $A$, Fractions of nitrogen discharged to coastal and inland waters. $B$, Fractions of nitrogen discharged to coastal and inland waters by nitrogen source.

Assumptions were also made in this study regarding the spatial location of nitrogen inputs and attenuation. For example, in estimating the locations of nitrogen inputs from septic systems, it was assumed that residents in areas served by public sewers did not have septic systems. Also, it was assumed that fertilizer was distributed uniformly across model cells by land use (inputs to agricultural cells were spatially homogeneous, as were inputs to turf and grass cells). The uncertainties in estimated nitrogen loads and travel times due to assumptions regarding nitrogen source location are likely relatively small compared to the uncertainty due to nitrogen loading and attenuation rates.

Other sources of uncertainty in the nitrogen transport model results are (1) the assumption that land-cover patterns did not change, (2) the exclusion of dispersion and adsorption transport processes, and (3) the limitations for the regional groundwater model discussed previously in the section "Limitations and Factors Affecting Model Predictions." Changes in land cover are likely relatively minor over the period of the nitrogen travel times, of which 75 percent are less than 5 years. The exclusion of dispersion likely has minor effects on the simulated transport times, based on work on Cape Cod done by Walter (2008). Adsorption would slow the transport of any nitrogen present as ammonia, but the ammonia fraction of total nitrogen in groundwater in the Niantic River watershed is relatively small (less than 5 percent in most cases) (Mullaney, 2015), suggesting that the effects of excluding adsorption are minor. 
A. Nitrogen loading to surface waters, both inland and coastal

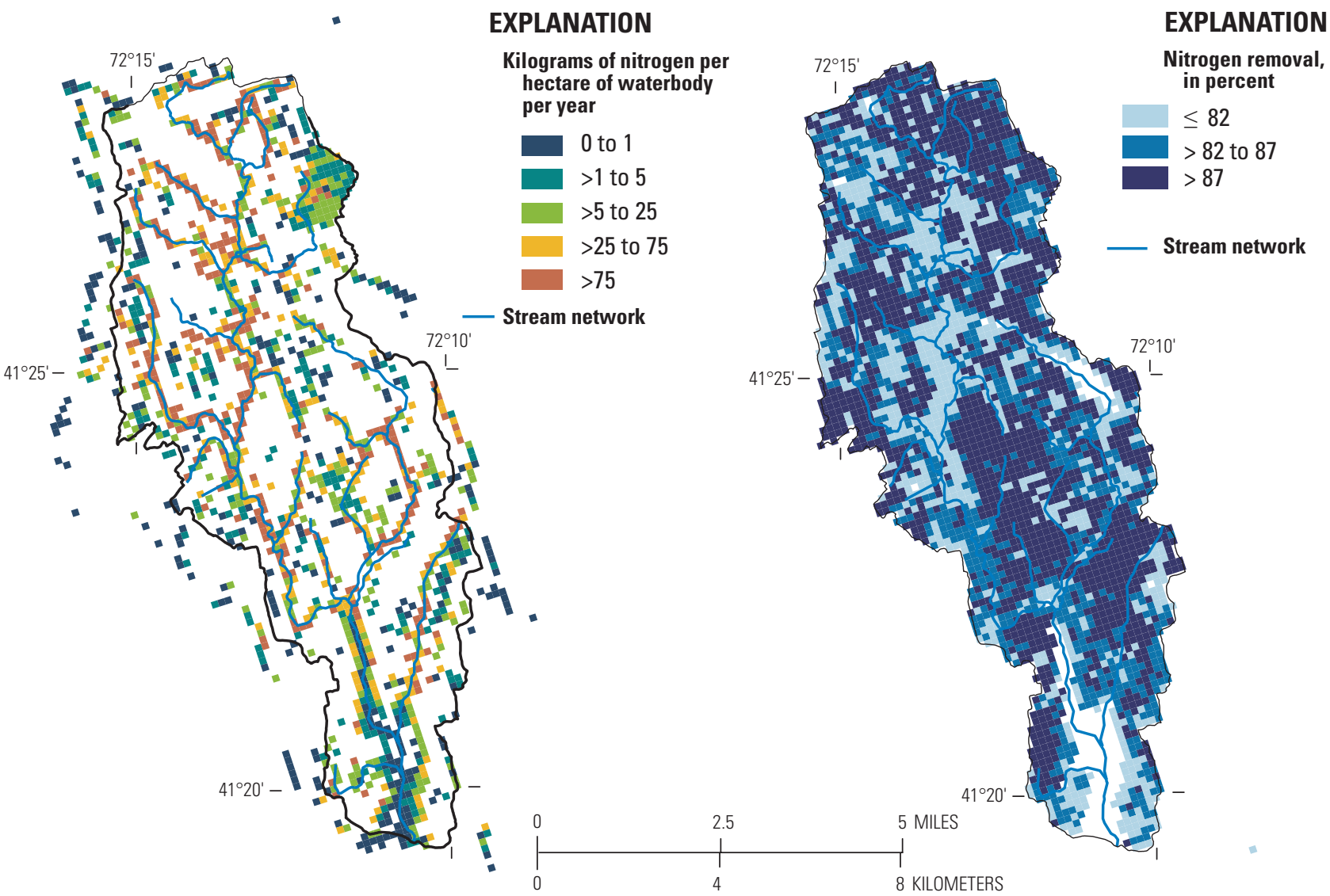

2011 Connecticut State Plane coordinate system

North American Datum of 1983

Figure 36. Simulated rates of nitrogen loading and removal within the Niantic River watershed, Connecticut. $A$, Rates of groundwater nitrogen loading to surface waters (coastal and inland) normalized to the discharge area for nitrogen sourced from within the Niantic River watershed. $B$, Percentages of nitrogen removal mapped to the source location. $\mathrm{kg}-\mathrm{N} / \mathrm{ha}$-waterbody/yr, kilograms of nitrogen per hectare of waterbody per year. 

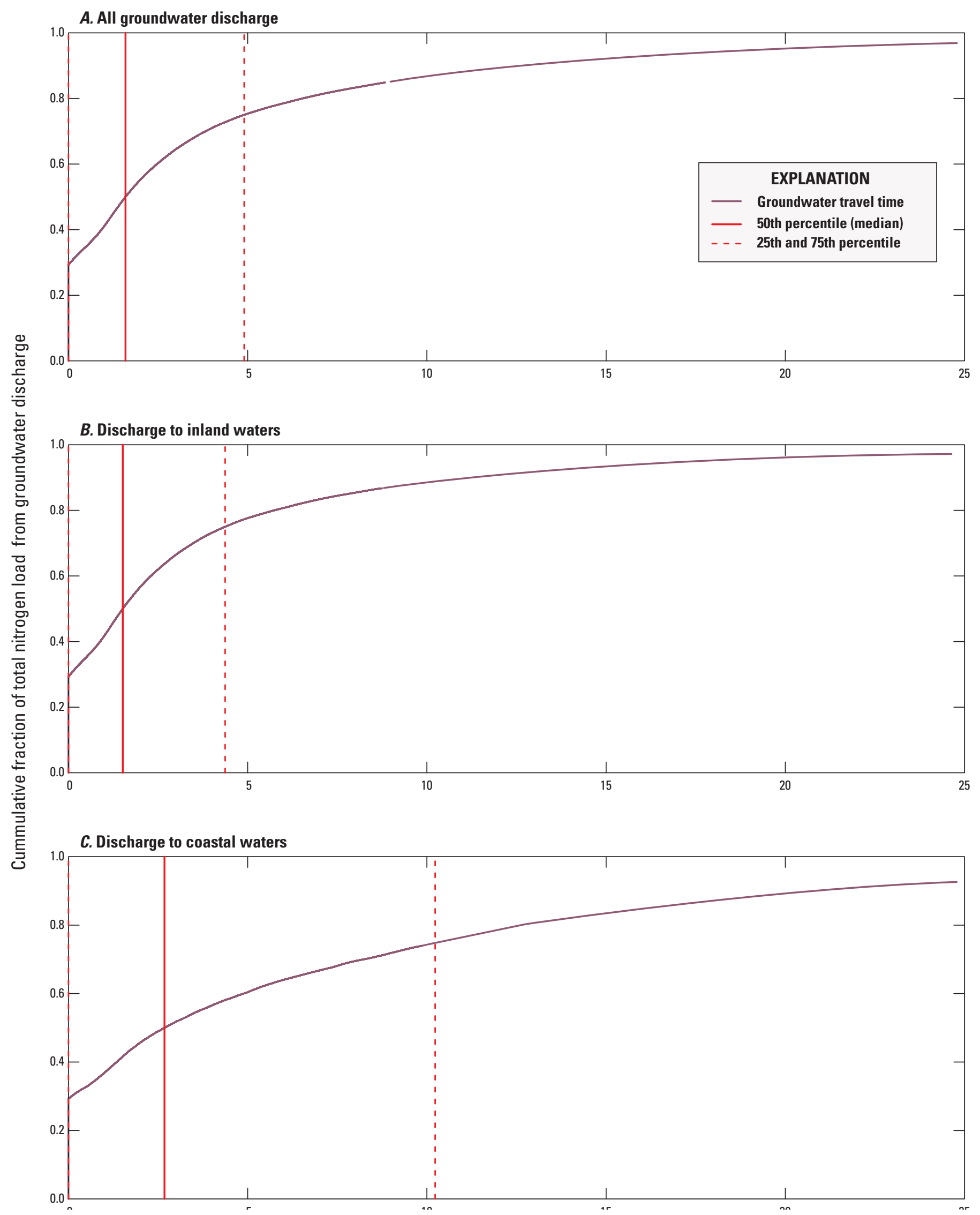

Figure 37. Simulated groundwater travel time for nitrogen by discharge location within the Niantic River watershed, Connecticut. $A$, All discharge locations. $B$, Inland waters. $C$, Coastal waters. 
A. Travel times: $<2$ years

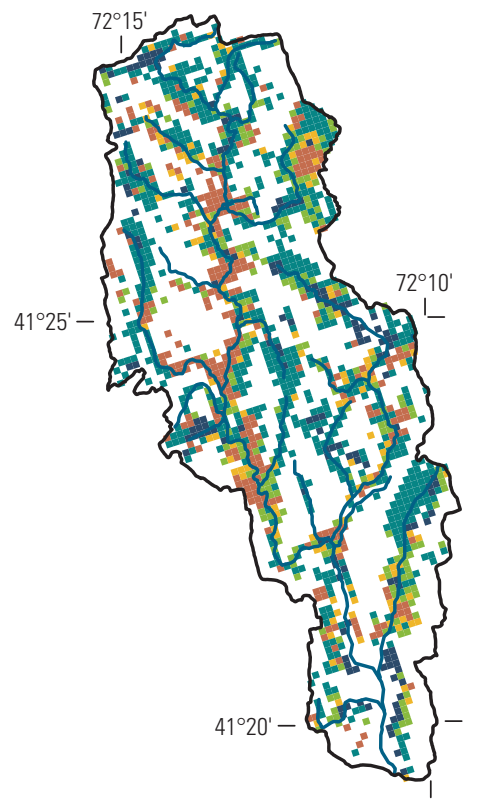

B. Travel times: 2 to 5 years

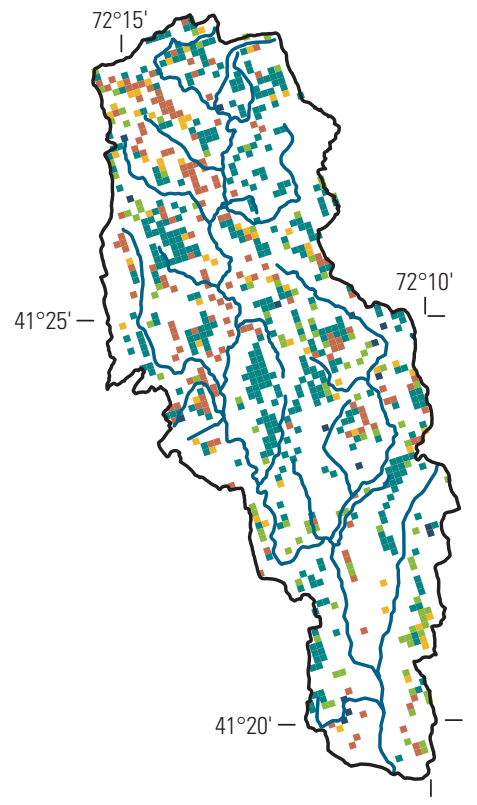

C. Travel times: $\mathbf{5}$ to 10 years

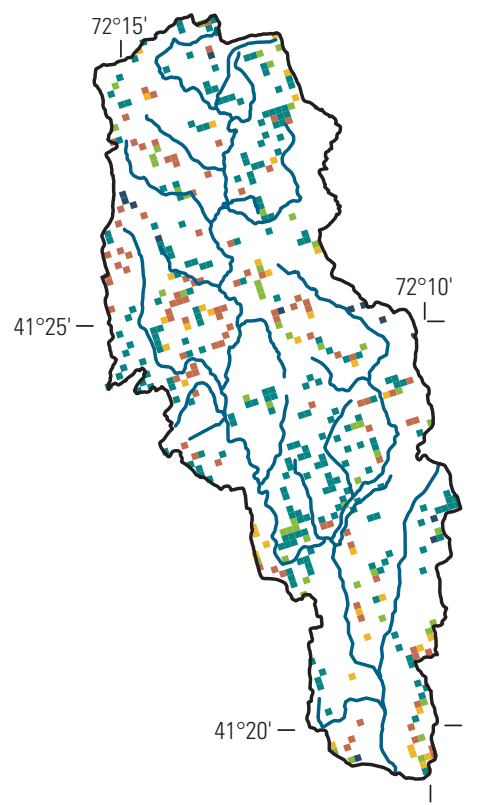

\section{Travel times: $>10$ years}

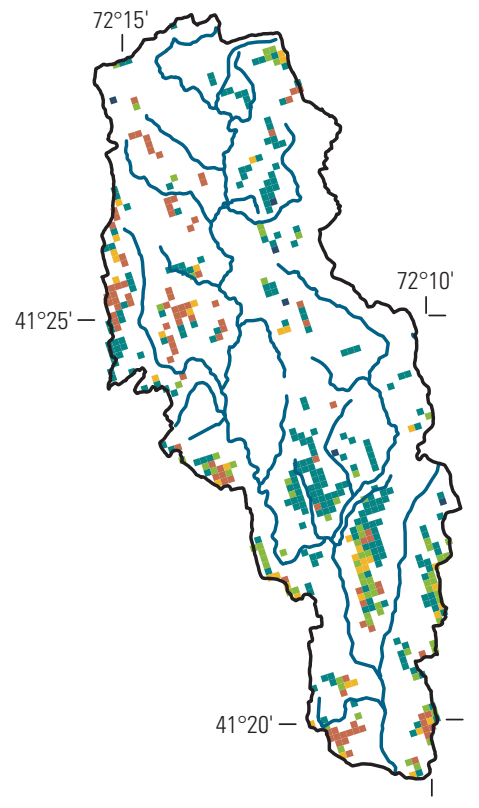

\section{EXPLANATION}

Kilograms of nitrogen per hectare per year

\section{0 to 5}

6 to10

11 to 15

16 to 20

$>20$

_ Stream network

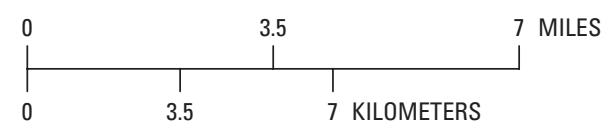

2011 Connecticut State Plane coordinate system North American Datum of 1983

Figure 38. Nitrogen inputs by groundwater travel time within the Niantic River watershed, Connecticut. $A$, Travel times $<2$ years. $B$, Travel times of $2-5$ years. $C$, Travel times of $5-10$ years. $D$, Travel times $>10$ years. 


\section{Summary}

Aquatic systems in and around Long Island Sound (LIS) provide a variety of ecological and economic benefits, but in some areas of the LIS aquatic ecosystems are degraded because of excess nitrogen. A substantial fraction of nitrogen inputs to the LIS are transported through the groundwater-flow system. Because groundwater travel times in surficial aquifers can exceed 100 years, multiyear lags are introduced between inputs at the water table and discharge to receiving waters. The U.S. Geological Survey, in cooperation with the Connecticut Department of Energy and Environmental Protection and the Long Island Sound Study, developed a steady-state groundwater model of the watersheds draining from the northern shore of the LIS for the purpose of calculating groundwater budgets and groundwater travel times to coastal waters.

The model was developed by using the MODFLOWNWT software and existing spatial data on aquifers, river networks, altitudes, land cover, groundwater recharge, and water use. Coastal waters were delineated on the basis of the National Wetland Inventory; all noncoastal waters were collectively termed "inland waters." Median groundwater recharge rates from natural sources, by sediment type, ranged from 7.87 to 66.92 centimeters per year $(\mathrm{cm} / \mathrm{yr})$, with a median of $29.38 \mathrm{~cm} / \mathrm{yr}$ across the study area. Septic return flows were simulated in 98,187 model cells, with rates ranging from less than $0.1 \mathrm{~cm} / \mathrm{yr}$ to $96 \mathrm{~cm} / \mathrm{yr}$, with a median of $1.35 \mathrm{~cm} / \mathrm{yr}$. Water supply withdrawals were simulated for 385 wells, with applied withdrawal rates ranging from 2.7 to 11,620 cubic meters per day $\left(\mathrm{m}^{3} / \mathrm{d}\right)$ and with a median of $40.51 \mathrm{~m}^{3} / \mathrm{d}$ $(10,700 \mathrm{gal} / \mathrm{d})$. Private well withdrawals, estimated by using population density and public water-supply service-area data, were simulated for 80,415 wells, with applied withdrawal rates ranging from 0.54 to $37.74 \mathrm{~m}^{3} / \mathrm{d}$ (143 to $\left.10,000 \mathrm{gal} / \mathrm{d}\right)$ and with a median of $1.03 \mathrm{~m}^{3} / \mathrm{d}(272 \mathrm{gal} / \mathrm{d})$, across the study area.

The model was developed with two horizontal grid resolutions. First, a coarser model with a horizontal resolution of 304.8-meters (m) (1,000-feet [ft]) was calibrated by using the PEST++ software, water levels in 65 wells with longterm records, stream altitudes from 477 streams, base flow at 14 streamgages that are relatively unaffected by withdrawals, and an error metric based on incorrectly simulated flooding and incorrectly simulated dry streams. The calibrated values were then used for a finer model with a $152.4-\mathrm{m}(500-\mathrm{ft})$ resolution. This approach resulted in a finer resolution simulation with decreased computational requirements for calibration. In the coarser, 304.8-m (1,000-ft) model, the mean absolute residual for well water levels was $4.7 \mathrm{~m}$, for stream altitudes was $1.1 \mathrm{~m}$, and for base-flow streamflow was $5,600 \mathrm{~m}^{3} / \mathrm{d}$ ( 2.3 cubic feet per second). About 91 percent of the terrestrial cells were correctly simulated as having the water table below land surface, and 92 percent of the cells representing streams were correctly simulated as having the water table above land surface, suggesting the model is sufficient for simulating broader flow patterns. In the finer, 152.4-m (500-ft) model, the mean absolute error for well water levels was $4.5 \mathrm{~m}$, for stream altitudes was $1.3 \mathrm{~m}$, and for base flow was $7,200 \mathrm{~m}^{3} / \mathrm{d}$ ( 2.9 cubic feet per second). In the finer resolution model, about 89 percent of the terrestrial cells were correctly simulated as having the water table below land surface, and nearly 90 percent of the cells representing streams were correctly simulated as having the water table above land surface, indicating the finer resolution model simulated the flow patterns as well as the calibrated coarser resolution model. The finer resolution model was used for all subsequent analyses because of its better representation of groundwater-discharge zones.

Groundwater budgets were compiled for the entire study area, for each HUC12 watershed and its adjacent coastal waters within the study area, and for 14 coastal-embayment watersheds. Across the entire study area, inflows were predominantly from natural recharge ( 98.8 percent), and a small (internally recycled) contribution was from septic return flows (1.2 percent). Outflows consisted of discharge to inland waters (90.6 percent), discharge to coastal waters ( 7.0 percent), and well withdrawals ( 2.4 percent). When groundwater budgets were compiled for each HUC12 watershed, the portion of groundwater discharging to coastal waters ranged from less than 1 to 66 percent of groundwater outflows, with a median of 13 percent, in HUC12 watersheds where coastal discharge occurred. Within coastal-embayment watersheds, the portion of groundwater discharging to coastal waters ranged from 2 to 56 percent, with a median of 15 percent.

Groundwater travel times were also compiled for the entire study area, for each HUC12 watershed and its adjacent coastal waters within the study area, and for 14 priority coastal embayments. Across the entire study area, the median groundwater travel time was 1.9 years, and the interquartile range was from 0.1 to 5.9 years. Travel times for groundwater discharging to coastal waters had a similar median (1.9 years) but a wider interquartile range ( 0 to 11.1 years). Median travel times for the HUC12 watersheds ranged from 0.9 to 53.5 years, with a median of 1.8 years. Among HUC12 watersheds that contain coastal areas, travel times for groundwater discharging to coastal waters ranged from less than 1 to 61.6 years, with a median of 2.8 years. Median travel times for groundwater discharging to coastal waters within the embayments ranged from less than 1 to 18.6 years, with a median of 2.3 years.

A sensitivity analysis was performed to assess the effects of uncertainty in subsurface porosity and of model-grid resolution on the simulated groundwater budgets and travel times. Increasing and decreasing the porosity by 50 percent did not change the distribution of groundwater between coastal waters, inland waters, and wells. Changing the porosity did, however, result in a linear, directly proportional change in the travel times, because of the same volume of water moving through a larger (with increased porosity) or smaller (with decreased porosity) space. Reducing the grid resolution from $304.8 \mathrm{~m}(1,000 \mathrm{ft})$ to $152.4 \mathrm{~m}(500 \mathrm{ft})$ and further to $76.2 \mathrm{~m}$ $(250 \mathrm{ft})$ decreased the fraction of groundwater discharging to coastal waters and increased the travel time for all groundwater discharge from a median of 0 years for the $304.8-\mathrm{m}$ 
$(1,000-\mathrm{ft})$ model to 1.6 years for the $152.4-\mathrm{m}(500-\mathrm{ft})$ model and to 1.8 years for the $76.2-\mathrm{m}$ ( $250-\mathrm{ft})$ model, likely because of finer resolution mapping of groundwater discharge areas.

The regional groundwater model was used for a demonstration study that simulated groundwater transport of nitrogen within the Niantic River watershed. Nitrogen inputs were estimated by using land-cover-based loading factors, and attenuation was estimated by using attenuation factors based on geologic zones and soil properties. By using the estimated nitrogen inputs and attenuation rates, groundwater nitrogen loads to selected surface water subbasins within the Niantic River watershed were simulated; the loads were similar to observed instantaneous stream loads. Based on this simulation, groundwater transports an estimated 22,000 kilograms of nitrogen per year (2.9 kilograms of nitrogen per hectare per year) to streams, rivers, and coastal waters within the Niantic River watershed. The resulting groundwater nitrogen load reflects a removal rate of 81 percent within the soil and groundwater system. Approximately 36 percent of discharging nitrogen is from atmospheric-deposition sources, 38 percent from fertilizers, and 26 percent from septic systems. In this simulation, most of the groundwater-transported nitrogen (88 percent) discharges to streams and rivers, and only 12 percent discharges directly to coastal waters. Subsurface travel times for groundwater-transported nitrogen ranged from less than 1 day to more than 100 years, with a median of 1.6 years.

\section{References Cited}

Anderson, M.P., 1997, Characterization of geological heterogeneity, in Dagan, G., and Neuman, S.P., eds., Subsurface flow and transport-A stochastic approach: New York, Cambridge University Press, p. 23-43, accessed December 16, 2020, at https://books.google.com/ books? id $=8 \mathrm{pqOuN}-\mathrm{G} 1 \mathrm{~A} 0 \mathrm{C} \&$ printsec $=$ frontcover $\# \mathrm{v}=$ onepage $\& q \& \mathrm{f}=$ false.

Ator, S.W., 2019, Spatially referenced models of streamflow and nitrogen, phosphorus, and suspended-sediment loads in streams of the northeastern United States: U.S. Geological Survey Scientific Investigations Report 2019-5118, 57 p., accessed June 25, 2021, at https://doi.org/10.3133/ $\operatorname{sir} 20195118$.

Bakker, M., Post, V., Langevin, C.D., Hughes, J.D., White, J.T., Starn, J.J., and Fienen, M.N., 2016, Scripting MODFLOW model development using Python and FloPy: Groundwater, v. 54, no. 5, p. 733-739, accessed June 25, 2021, at https://doi.org/10.1111/gwat.12413.
Barclay, J.R., and Mullaney, J.R., 2021a, MODFLOW-NWT and MODPATH groundwater flow models of steady-state conditions in coastal Connecticut and adjacent areas of New York and Rhode Island, as well as a nitrogen transport model of the Niantic River watershed: U.S. Geological Survey data release, https://doi.org/10.5066/P9BLHPIT.

Barclay, J.R., and Mullaney, J.R., 2021b, Summary data on groundwater budgets and travel times for watersheds on the north shore of Long Island Sound: U.S. Geological Survey data release, https://doi.org/10.5066/P91TQ895.

Befus, K.M., Kroeger, K.D., Smith, C.G., and Swarzenski, P.W., 2017, The magnitude and origin of groundwater discharge to eastern U.S. and Gulf of Mexico coastal waters: Geophysical Research Letters, v. 44, no. 20, p. 10396-10406, accessed June 25, 2021, at https://doi.org/ 10.1002/2017GL075238.

Billen, G., Ramarson, A., Thieu, V., Théry, S., Silvestre, M., Pasquier, C., Hénault, C., and Garnier, J., 2018, Nitrate retention at the river-watershed interface-A new conceptual modeling approach: Biogeochemistry, v. 139, p. 31-51, accessed June 25, 2021, at https://doi.org/10.1007/s10533018-0455-9.

Center for Land Use Education and Research (CLEAR), 2016, Long Island Sound 2010 land cover: CLEAR, Connecticut's Changing Landscape Land Cover dataset, accessed December 5, 2019, at https:/clear.uconn.edu/ projects/landscape/download/LIS_landcover2010_v2-03_ ctstp83.zip.

Connecticut Department of Energy and Environmental Protection [CT DEEP], 2012, Aquifer Protection Areas: Connecticut Department of Energy and Environmental Protection dataset, accessed March 8, 2019, at ftp://ftp.state.ct.us/pub/dep/gis/shapefile_format_zip/ Aquifer_Protection_Area_shp.zip.

Connecticut Department of Energy and Environmental Protection [CT DEEP], 2019a, Connecticut's Second Generation Nitrogen Strategy-Long Island Sound, 2017-2022: Connecticut Department of Energy and Environmental Protection, 19 p., accessed May 4, 2020, at https://portal.ct.gov/-/media/DEEP/water/lis_water quality/nitrogen_control_program/2ndGenNitrogenStrate gypdf.pdf?la=en.

Connecticut Department of Energy and Environmental Protection [CT DEEP], 2019b, Sewer service areas 1998 (Sewer_Service.shp): Connecticut Department of Energy and Environmental Protection dataset, accessed July 19, 2021, at https://ct-deep-gis-open-data-website-ctdeep.hub.a rcgis.com/datasets/sewer-service-area-1998. 
Connecticut Department of Public Health, 2013, Public water supply service areas in Connecticut: State of Connecticut dataset, accessed March 5, 2019, at https://www.arcgis.com/ home/item.html?id=b5a6750a85cd49ffa800e1aab88db95c.

Connecticut Office of Policy and Management, 2019, Public water service areas 2017 (PublicWaterService2017.shp): State of Connecticut dataset, accessed December 2, 2019, at ftp://ftp.state.ct.us/pub/opm/TCDPP/C\&D\%20Plan\%20 GIS/GIS/OPM_DRAFT_C\&D_Data_Web/WaterService/.

Department of City Planning, 2019, Boundaries of boroughs: City of New York dataset, accessed March 4, 2019, at https://data.cityofnewyork.us/City-Government/BoroughBoundaries/tqmj-j8zm.

Dieter, C.A., Linsey, K.S., Caldwell, R.R., Harris, M.A., Ivahnenko, T.I., Lovelace, J.K., Maupin, M.A., and Barber, N.L., 2018, Estimated use of water in the United States county-level data for 2015: U.S. Geological Survey data release, accessed June 25, 2021, at https://doi.org/10.5066/ F7TB15V5.

Domenico, P., and Schwartz, F., 1997, Physical and chemical hydrogeology 2nd ed.: New York, Wiley, 506 p.

Division of Water-Bureau of Water Resource Management, 2018, NYS water withdrawals - Excludes DEC Region 1 (NYSDEC): State of New York, Division of Water-Bureau of Water Resource Management dataset, accessed March 4 2019, at https://gis.ny.gov/gisdata/inventories/details.cfm? $\mathrm{DSID}=1265$.

Falcone, J.A., 2011, GAGES-II: Geospatial Attributes of Gages for Evaluating Streamflow: U.S. Geological Survey dataset, accessed June 25, 2021, at https://doi.org/10.3133/ 70046617.

Falcone, J.A., 2016, U.S. block-level population density rasters for 1990, 2000, and 2010: U.S. Geological Survey data release, accessed June 24, 2021, at https://doi.org/10.5066/ F74J0C6M.

Gold, A.J., DeRagon, W.R., Sullivan, W.M., and Lemunyon, J.L., 1990, Nitrate-nitrogen losses to groundwater from rural and suburban land uses: Journal of Soil and Water Conservation, v. 45, no. 2, p. 305-310, accessed June 24, 2021, at https://www.jswconline.org/content/45/2/305.

Guillard, K., and Fitzpatrick, R., 2011, Nitrogen fertilizer reductions on coastal lawns through training and education: University of Connecticut, final report for EPA Clean Water Act Section 319 Grant, report number 08-10, 29 p.

Gustard, A., Bullock, A., and Dixon, J., 1992, Low flow estimation in the United Kingdom: Institute of Hydrology report 108, [variously paged; $292 \mathrm{p}$ ].
Haj, A.E., Soller, D.R., Reddy, J.E., Kauffman, L.J., Yager, R.M., and Buchwald, C.A., 2018, Hydrogeologic framework for characterization and occurrence of confined and unconfined aquifers in quaternary sediments in the glaciated conterminous United States-A digital map compilation and database: U.S. Geological Survey Data Series 1090, 31 p., accessed June 24, 2021, at https://doi.org/10.3133/ds1090.

Han, D., Currell, M.J., Cao, G., and Hall, B., 2017, Alterations to groundwater recharge due to anthropogenic landscape change: Journal of Hydrology, v. 554, p. 545-557, accessed June 24, 2021, at https://doi.org/10.1016/j.jhydrol. 2017.09.018.

Harbaugh, A.W., 2005, MODFLOW-2005, The U.S. Geological Survey modular ground-water model-The ground-water flow process: U.S. Geological Survey Techniques and Methods, book 6, chap. A16, 253 p., accessed June 24, 2021, at https://doi.org/10.3133/tm6A16.

Helton, A.M., Poole, G.C., Meyer, J.L., Wollheim, W.M., Peterson, B.J., Mulholland, P.J., Bernhardt, E.S., Stanford, J.A., Arango, C., Ashkenas, L.R., Cooper, L.W., Dodds, W.K., Gregory, S.V., Hall, R.O., Jr., Hamilton, S.K., Johnson, S.L., McDowell, W.H., Potter, J.D., Tank, J.L., Thomas, S.M., Valett, H.M., Webster, J.R., and Zeglin, L., 2011, Thinking outside the channel-Modeling nitrogen cycling in networked river ecosystems: Frontiers in Ecology and the Environment, v. 9, no. 4, p. 229-238, accessed June 24, 2021, at https://doi.org/10.1890/080211.

Hibbs, B.J., and Sharp, J.M., Jr., 2012, Hydrogeological impacts of urbanization: Environmental \& Engineering Geoscience, v. 18, no. 1, p. 3-24, accessed June 24, 2021, at https://doi.org/10.2113/gseegeosci.18.1.3.

Homer, C.G., Dewitz, J.A., Yang, L., Jin, S., Danielson, P., Xian, G., Coulston, J., Herold, N.D., Wickham, J., and Megown, K., 2015, Completion of the 2011 National Land Cover Database for the conterminous United StatesRepresenting a decade of land cover change information: Photogrammetric Engineering and Remote Sensing, v. 81, no. 5, p. 345-354, accessed June 24, 2021, at https://www. ingentaconnect.com/content/asprs/pers/2015/00000081/ 00000005/art00002.

Horton, J.D., 2017, The State Geologic Map Compilation (SGMC) geodatabase of the conterminous United States (ver. 1.1, August 2017): U.S. Geological Survey data release, accessed July 22, 2019, at https://doi.org/10.5066/ F7WH2N65.

Kauffman, G.J., Belden, A.C., Vonck, K.J., and Homsey, A.R., 2009, Link between impervious cover and base flow in the White Clay Creek Wild and Scenic Watershed in Delaware: Journal of Hydrologic Engineering, v. 14, no. 4, p. 324-334, accessed June 24, 2021, at https://doi.org/10.1061/ (ASCE)1084-0699(2009)14:4(324). 
Kazmann, R.G., 1948, The induced infiltration of river water to wells: Eos, Transactions, American Geophysical Union, v. 29, no. 1, p. 85-92, accessed June 24, 2021, at https://doi.org/10.1029/TR029i001p00085.

Latimer, J.S., and Rego, S.A., 2010, Empirical relationship between eelgrass extent and predicted watershed-derived nitrogen loading for shallow New England estuaries: Estuarine, Coastal and Shelf Science, v. 90, no. 4, p. 231-240, accessed June 24, 2021, at https://doi.org/ 10.1016/j.ecss.2010.09.004.

Lerner, D.N., 1990, Groundwater recharge in urban areas: Atmospheric Environment. Part B, Urban Atmosphere, v. 24, no. 1, p. 29-33, accessed June 24, 2021, at https://doi.org/10.1016/0957-1272(90)90006-G.

Lerner, D.N., 2002, Identifying and quantifying urban recharge - A review: Hydrogeology Journal, v. 10, no. 1, p. 143-152, accessed June 24, 2021, at https://doi.org/ 10.1007/s10040-001-0177-1.

Levin, S.B., Olson, S.A., Nielsen, M.G., and Granato, G.E., 2018, The Connecticut Streamflow and Sustainable Water Use Estimator-A decision-support tool to estimate water availability at ungaged stream locations in Connecticut: U.S. Geological Survey Scientific Investigations Report 2018-5135, 34 p., accessed June 24, 2021, at https://doi.org/ $10.3133 /$ sir20185135.

Long Island Sound Study [LISS], 2015, Long Island Sound study comprehensive conservation and management plan 2015-Returning the urban sea to abundance: Long Island Sound Study, 70 p., accessed December 2, 2019, at https://longislandsoundstudy.net/2015/09/2015comprehensive-conservation-and-management-plan/.

Long Island Sound Resource Center and U.S. Geological Survey, 2004, Thickness of glacial sediments in Connecticut and the Long Island Sound Basin polygon: State of Connecticut, Department of Environmental Protection dataset, accessed January 14, 2020, at ftp://ftp.state.ct.us/ pub/dep/gis/shapefile_format_zip/Glacial_Sediment_ Thickness_shp.zip.

Lorenz, D.L., 2017, DVstats: Functions to manipulate dailyvalues data (ver. 0.3.4): U.S. Geological Survey R software package, accessed June 24, 2021, at https://rdrr.io/github/ USGS-R/DVstats/.
Lyford, F.P., Carlson, C.S., Brown, C.J., and Starn, J.J., 2007, Hydrogeologic setting and ground-water flow simulations of the Pomperaug River Basin regional study area, Connecticut, section 6 of Paschke, S.S., ed., Hydrogeologic settings and ground-water flow simulations for regional studies of the transport of anthropogenic and natural contaminants to public-supply wells - Studies begun in 2001: U.S. Geological Survey Professional Paper 1737-A, p. 6-1-6-26, accessed June 24, 2021, at https://doi.org/ 10.3133/pp1737A.

Masterson, J.P., and Granato, G.E., 2013, Numerical simulation of groundwater and surface-water interactions in the Big River Management Area, central Rhode Island: U.S. Geological Survey Scientific Investigations Report 2012-5077, 64 p., accessed June 24, 2021, at https://doi.org/ $10.3133 /$ sir20125077.

Maupin, M.A., Kenny, J.F., Hutson, S.S., Lovelace, J.K., Barber, N.L., and Linsey, K.S., 2014, Estimated use of water in the United States in 2010: Circular 1405, 64 p., accessed June 24, 2021, at https://doi.org/10.3133/cir1405.

McGuire, K.J., and McDonnell, J.J., 2006, A review and evaluation of catchment transit time modeling: Journal of Hydrology, v. 330, no. 3-4, p. 543-563, accessed June 24, 2021, at https://doi.org/10.1016/j.jhydrol.2006.04.020.

Meals, D.W., Dressing, S.A., and Davenport, T.E., 2010, Lag time in water quality response to best management practices-A review: Journal of Environmental Quality, v. 39, no. 1, p. 85-96, accessed June 24, 2021, at https://doi.org/10.2134/jeq2009.0108.

Melvin, R.L., Stone, B.D., Stone, J.R., and Trask, N.J., 1992, Hydrogeology of thick till deposits in Connecticut: U.S. Geological Survey Open-File Report 92-43, 43 p., accessed June 24, 2021, at https://doi.org/10.3133/ofr9243.

Mulholland, P.J., Helton, A.M., Poole, G.C., Hall, R.O., Hamilton, S.K., Peterson, B.J., Tank, J.L., Ashkenas, L.R., Cooper, L.W., Dahm, C.N., Dodds, W.K., Findlay, S.E.G., Gregory, S.V., Grimm, N.B., Johnson, S.L., McDowell, W.H., Meyer, J.L., Valett, H.M., Webster, J.R., Arango, C.P., Beaulieu, J.J., Bernot, M.J., Burgin, A.J., Crenshaw, C.L., Johnson, L.T., Niederlehner, B.R., O’Brien, J.M., Potter, J.D., Sheibley, R.W., Sobota, D.J., and Thomas, S.M., 2008, Stream denitrification across biomes and its response to anthropogenic nitrate loading: Nature, v. 452 , no. 7184 , p. 202-205, accessed June 24, 2021, at https://doi.org/ 10.1038 /nature 06686 .

Mullaney, J.R., 2013, Nutrient concentrations and loads and Escherichia coli densities in tributaries of the Niantic River estuary, southeastern Connecticut, 2005 and 2008-2011: Scientific Investigations Report 2013-5008, 27 p., accessed June 24, 2021, at https://doi.org/10.3133/sir20135008. 
Mullaney, J.R., 2015, Evaluation of the effects of sewering on nitrogen loads to the Niantic River, southeastern Connecticut, 2005-2011: Scientific Investigations Report 2015-5011, 30 p., accessed June 24, 2021, at https://doi.org/ 10.3133/sir20155011.

National Atmospheric Deposition Program, 2018, Total deposition maps (ver. 2018.01): National Atmospheric Deposition Program dataset, accessed December 2, 2019, at http://nadp.slh.wisc.edu/committees/tdep/tdepmaps/.

New York State Department of Environmental Conservation, 2014, New York State design standards for intermediate sized wastewater treatment systems: New York State Department of Environmental Conservation, Division of Water, [variously paged; 269 p.], accessed June 5, 2020, at https://www.dec.ny.gov/docs/water_pdf/2014designstd.pdf.

New York State Museum and New York State Geological Survey, 1999, Surficial geology_Lower Hudson: New York State Museum Technology Center digital dataset [corresponds with original 1989 map sheet], scale 1:250,000, accessed June 4, 2019, at http://www.nysm.nysed.gov/ research-collections/geology/gis.

Niswonger, R.G., Panday, S., and Ibaraki, M., 2011, MODFLOW-NWT, A Newton formulation for MODFLOW-2005: U.S. Geological Survey Techniques and Methods, 6-A37, 44 p., accessed June 24, 2021, at https://doi.org/10.3133/tm6A37.

Niswonger, R.G., and Prudic, D.E., 2005, Documentation of the Streamflow-Routing (SFR2) Package to include unsaturated flow beneath streams - A modification to SFR1: U.S. Geological Survey Techniques and Methods, book 6, chap. A13, 57 p., accessed June 24, 2021, at https://doi.org/ 10.3133/tm6A13.

Olcott, P.G., 1995, Connecticut, Maine, Massachusetts, New Hampshire, New York, Rhode Island, Vermont, segment 12 of Groundwater atlas of the United States: U.S. Geological Survey Hydrologic Investigations Atlas, v. 130-M, p. M1-M28, accessed June 24, 2021, at https://doi.org/ 10.3133/ha730M.

Oliphant, T.E., 2007, Python for scientific computing: Computing in Science \& Engineering, v. 9, no. 3, p. 10-20, accessed June 24, 2021, at https://doi.org/10.1109/ MCSE.2007.58.

Open Sewer Atlas, NYC, 2016, Sewersheds: Open Sewer Atlas NYC dataset, accessed June 30, 2021, at http $\mathrm{s}: / /$ openseweratlas.tumblr.com/data.

Pollock, D.W., 2012, User guide for MODPATH version 6-A particle-tracking model for MODFLOW: U.S. Geological Survey Techniques and Methods, book 6, chap. A41, 58 p., accessed June 24, 2021, at https://doi.org/10.3133/tm6A41.
PRISM Climate Group, 2012, 30 yr mean precipitation (PRISM_ppt_30yr_normal_800mM2_annual_bil. bil): Oregon State University, Northwest Alliance for Computational Science \& Engineering dataset, accessed September 27, 2019, at http://prism.oregonstate.edu.

R Core Team, 2019, R-A language and environment for statistical computing (ver. 3.6.0): The R Foundation software release, accessed July 1, 2019, at http://www.R-project.org.

Radcliffe, D.E., and Bradshaw, J.K., 2014, Model test of proposed loading rates for onsite wastewater treatment systems: Soil Science Society of America Journal, v. 78, no. 1, p. 97-107, accessed June 20, 2021, at https://doi.org/ 10.2136/sssaj2013.07.0257.

Reisinger, A.J., Tank, J.L., Rosi-Marshall, E.J., Hall, R.O., Jr., and Baker, M.A., 2015, The varying role of water column nutrient uptake along river continua in contrasting landscapes: Biogeochemistry, v. 125, no. 1, p. 115-131, accessed June 24, 2021, at https://doi.org/10.1007/s10533015-0118-z.

Rhode Island Geographic Information System [RIGIS], 1989, Rhode Island glacial deposits - s44ggl88: Rhode Island Geographic Information System (RIGIS) Data Distribution System, accessed July 13, 2018, at https://www.rigis.org/ datasets/glacial-deposits.

Rhode Island Geographic Information System [RIGIS], 1995, Water supply districts - s44uwd95: Rhode Island Geographic Information System (RIGIS) Data Distribution System, accessed March 4, 2019, at https://www.rigis.org/ datasets/water-supply-districts.

Rhode Island Geographic Information System [RIGIS], 2012, Rhode Island sewered areas - sewerAreas12: Rhode Island Geographic Information System (RIGIS) Data Distribution System, accessed March 4, 2019, at https://www.rigis.org/ datasets/sewered-areas.

Rutledge, A.T., 1998, Computer programs for describing the recession of ground-water discharge and for estimating mean ground-water recharge and discharge from streamflow records-update: U.S. Geological Survey Water-Resources Investigations Report 98-4148, 43 p., accessed June 24, 2021, at https://doi.org/10.3133/wri984148.

Sanford, W.E., Pope, J.P., Selnick, D.L., and Stumvoll, R.F., 2012, Simulation of groundwater flow in the shallow aquifer system of the Delmarva Peninsula, Maryland and Delaware: U.S. Geological Survey Open-File Report 2012-1140, 58 p., accessed June 24, 2021, at https://doi.org/ $10.3133 /$ ofr20121140.

Seaber, P.R., Kapinos, F.P., and Knapp, G.L., 1987, Hydrologic unit maps: U.S. Geological Survey Water Supply Paper 2294, 63 p., 1 pl., accessed June 25, 2021, at http://pubs.er.usgs.gov/publication/wsp2294. 
Shaffer, K.H., and Runkle, D.L., 2007, Consumptive water-use coefficients for the Great Lakes Basin and climatically similar areas: U.S. Geological Survey Scientific Investigations Report 2007-5197, 191 p., accessed June 24, 2021, at https://doi.org/10.3133/sir20075197.

Shimizu, M., Wentz, E.A., Merson, J., Kellogg, D.Q., and Gold, A.J., 2018, Modeling anthropogenic nitrogen flow for the Niantic River catchment in coastal New England: Landscape Ecology, v. 33, no. 8, p. 1385-1398, accessed June 24, 2021, at https://doi.org/10.1007/s10980018-0667-3.

Soil Survey Staff, 2017a, The Gridded Soil Survey Geographic (gSSURGO) Database for Connecticut (FY 2018 official release): United States Department of Agriculture, Natural Resources Conservation Service, accessed June 6, 2018, at https://gdg.sc.egov.usda.gov/.

Soil Survey Staff, 2017b, The Gridded Soil Survey Geographic (gSSURGO) Database for New York (FY 2018 official release): United States Department of Agriculture, Natural Resources Conservation Service, accessed June 6, 2018, at https://gdg.sc.egov.usda.gov/.

Soil Survey Staff, 2017c, The Gridded Soil Survey Geographic (gSSURGO) Database for Rhode Island (FY 2018 official release): United States Department of Agriculture, Natural Resources Conservation Service, accessed June 6, 2018, at https://gdg.sc.egov.usda.gov/.

Starn, J.J., and Belitz, K., 2018, Regionalization of groundwater residence time using metamodeling: Water Resources Research, v. 54, no. 9, p. 6357-6373, accessed June 24, 2021, at https://doi.org/10.1029/2017WR021531.

Starn, J.J., and Brown, C.J., 2007, Simulations of groundwater flow and residence time near Woodbury, Connecticut: U.S. Geological Survey Scientific Investigations Report 2007-5210, 56 p., accessed June 24, 2021, at https://doi.org/ 10.3133/sir20075210.

Starn, J.J., and Carlson, C.S., 2018, Supporting datasets used in the General Groundwater-Model Construction System Version 0.1: U.S. Geological Survey data release, accessed June 24, 2021, at https://doi.org/10.1029/2017WR021531.

Stone, J.R., Schafer, J.P., London, E.H., DiGiacomo-Cohen, M.L., Lewis, R.S., and Thompson, W.B., 2005, Quaternary geologic map of Connecticut and Long Island Sound Basin: U.S. Geological Survey Scientific Investigations Map 2784, 2 sheets, scale 1:125,000, accessed August 27, 2021, at https://doi.org/10.3133/sim2784.

Stone, J.R., Schafer, J.P., London, E.H., and Thompson, W.B., 1992, Surficial materials map of Connecticut: Reston, Va., U.S. Geological Survey, 2 sheets, scale 1:125,000, accessed June 24, 2021, at https://doi.org/10.3133/70046712.
Stony Brook University Center for Survey Research, 2006, Public perception survey of Long Island Sound watershed residents: Stony Brook University Center for Survey Research, 54 p., accessed June 5, 2020, at http://longislandsoundstudy.net/wp-content/uploads/2010/ 03/LIS.Public.Perception.Survey2006.pdf.

Thompson, C., Smith, L., and Maji, R., 2007, Hydrogeological modeling of submarine groundwater discharge on the continental shelf of Louisiana: Journal of Geophysical Research. Oceans, v. 112, no. C3., 13 p., accessed June 24, 2021, at https://doi.org/10.1029/2006JC003557.

U.S. Department of Agriculture, Natural Resources Conservation Service, 2019, National soil survey handbook, title 430, accessed July 29, 2021, at https://directives. sc.egov.usda.gov/OpenNonWebContent.aspx? content= 44385.wba.

U.S. Environmental Protection Agency, 2002, Onsite wastewater treatment systems manual: U.S. Environmental Protection Agency EPA/625/R-00/008, [variously paged, 367 p.], accessed February 2, 2021, at https://www.epa.gov/ sites/production/files/2015-06/documents/2004_07_07_ septics_septic_2002_osdm_all.pdf.

U.S. Environmental Protection Agency and U.S. Geological Survey, 2012, National Hydrography Dataset PlusNHDPlus (ver. 2.10): U.S. Environmental Protection Agency and U.S. Geological Survey dataset, accessed June 24, 2021, at https://www.epa.gov/waterdata/nhdplusnational-hydrography-dataset-plus.

U.S. Fish and Wildlife Service, 2018, National wetlands inventory: U.S. Fish and Wildlife Service dataset, accessed April 29, 2019, at https://www.fws.gov/wetlands/.

U.S. Geological Survey, 2002, Aquifers of alluvial and glacial origin: U.S. Geological Survey dataset, accessed December 19, 2019, at https://water.usgs.gov/ogw/aquifer/ alluv-glac/alvaqfp025_nt00171.tar.gz.

U.S. Geological Survey, 2003, Principal aquifers of the 48 conterminous United States, Hawaii, Puerto Rico, and the U.S. Virgin Islands: U.S. Geological Survey digital data, accessed December 19, 2019, at https://water.usgs.gov/GIS/ metadata/usgswrd/XML/aquifers_us.xml.

U.S. Geological Survey, 2020, USGS water data for the Nation: U.S. Geological Survey National Water Information System database, accessed February 7, 2020, at https://doi.org/10.5066/F7P55KJN. 
U.S. Geological Survey, Connecticut Department of Energy and Environmental Protection, and Geological and Natural History Survey, 2005, Connecticut Quaternary geology and surficial materials polygon: Connecticut Department of Energy and Environmental Protection dataset, accessed August 27, 2021, at https://ct-deep-gis-open-data-websitectdeep.hub.arcgis.com/datasets/CTDEEP:quaternarygeology/about.

Valiela, I., Collins, G., Kremer, J., Lajtha, K., Geist, M., Seely, B., Brawley, J., and Sham, C.H., 1997, Nitrogen loading from coastal watersheds to receiving estuaries- $-\mathrm{New}$ method and application: Ecological Applications, v. 7, no. 2, p. 358-380, accessed June 24, 2021, at https://doi.org/ 10.1890/1051-0761(1997)007[0358:NLFCWT]2.0.CO;2.

Vaudrey, J.M.P., 2008, Establishing restoration objectives for eelgrass in Long Island Sound-Part II-Case studies: Final grant report to the Connecticut Department of Environmental Protection, Bureau of Water Protection and Land Reuse and the U.S. Environmental Protection Agency, 59 p., accessed May 19, 2020, at http://www.lisrc. uconn.edu/eelgrass/documents/LIS\%20Eelgrass\% 20Case\%20Studies.pdf.

Vaudrey, J.M.P., Kremer, J.N., Branco, B.F., and Short, F.T., 2010, Eelgrass recovery after nutrient enrichment reversal: Aquatic Botany, v. 93, no. 4, p. 237-243, accessed June 24, 2021, at https://doi.org/10.1016/j.aquabot.2010.08.005.

Vaudrey, J.M.P., Yarish, C., Kim, J.K., Pickerell, C., and Brousseau, L., 2016a, Comparative analysis and model development for determining the susceptibility to eutrophication of Long Island Sound embayments: Connecticut Sea Grant Project report R/CE-34-CTNY, 46 p., accessed February 18, 2020, at https://vaudrey.lab.uconn.edu/wpcontent/uploads/sites/1663/2017/02/Vaudrey_R-CE-34CTNY_FinalReport_2016.pdf.

Vaudrey, J.M.P., Yarish, C., Kim, J.K., Pickerell, C., and Brousseau, L., 2016b, Long Island Sound nitrogen loading model, 2016 version: Groton, Conn., University of Connecticut, accessed December 9, 2019, at https:/vaudrey.lab.uconn.edu/wp-content/uploads/sites/ 1663/2016/02/LongIslandSound_NLoadingModel_ v2016.xlsx.

Walter, D.A., 2008, Use of numerical models to simulate transport of sewage-derived nitrate in a coastal aquifer, central and western Cape Cod, Massachusetts: U.S. Geological Survey Scientific Investigations Report 2007-5259, 41 p., accessed June 24, 2021, at https://doi.org/10.3133/ $\operatorname{sir} 20075259$.
Walter, D.A., McCobb, T.D., and Fienen, M.N., 2019, Use of a numerical model to simulate the hydrologic system and transport of contaminants near Joint Base Cape Cod, western Cape Cod, Massachusetts: U.S. Geological Survey Scientific Investigations Report 2018-5139, 98 p., accessed June 24, 2021, at https://doi.org/10.3133/sir20185139.

Welter, D.E., White, J.T., Hunt, R.J., and Doherty, J.E., 2015, Approaches in highly parameterized inversion-PEST++ Version 3, a Parameter ESTimation and uncertainty analysis software suite optimized for large environmental models: U.S. Geological Survey Techniques and Methods, book 7 , chap. C12, 54 p., accessed June 24, 2021, at https://doi.org/ $10.3133 / \mathrm{tm} 7 \mathrm{C} 12$.

Westchester County Department of Planning, 2015a, Sewer districts: Westchester County dataset, accessed March 4, 2019, at https://giswww.westchestergov.com/download/ wcsewdst.zip.

Westchester County Department of Planning, 2015b, Water districts: Westchester County dataset, accessed March 4, 2019, at https://giswww.westchestergov.com/download/ wcwatdst.zip.

Westenbroek, S.M., Kelson, V.A., Dripps, W.R., Hunt, R.J., and Bradbury, K.R., 2010, SWB-A modified Thornthwaite-Mather Soil-Water-Balance code for estimating groundwater recharge: U.S. Geological Survey Techniques and Methods, book 6, chap. A31, 60 p., accessed June 24, 2021, at https://doi.org/10.3133/tm6A31.

Wittenberg, H., and Aksoy, H., 2010, Groundwater intrusion into leaky sewer systems: Water Science and Technology, v. 62 , no. 1, p. 92-98, accessed June 24, 2021, at https://doi.org/10.2166/wst.2010.287.

Wollheim, W.M., Mulukutla, G.K., Cook, C., and Carey, R.O., 2017, Aquatic nitrate retention at river network scales across flow conditions determined using nested in situ sensors: Water Resources Research, v. 53, no. 11, p. 9740-9756, accessed June 24, 2021, at https://doi.org/ 10.1002/2017WR020644.

Yager, R.M., Kauffman, L.J., Buchwald, C.A., Westenbroek, S.M., and Reddy, J.E., 2018a, Digital products from a hydrogeologic framework for Quaternary sediments within the glaciated conterminous United States: U.S. Geological Survey data release, accessed June 24, 2021, at https://doi.org/10.5066/F7HH6J8X.

Yager, R.M., Kauffman, L.J., Soller, D.R., Haj, A.E., Heisig, P.M., Buchwald, C.A., Westenbroek, S.M., and Reddy, J.E., 2018b, Characterization and occurrence of confined and unconfined aquifers in Quaternary sediments in the glaciated conterminous United States: U.S. Geological Survey Scientific Investigations Report 2018-5091, 90 p., accessed June 24, 2021, at https://doi.org/10.3133/sir20185091. 


\section{Appendix 1. Comparison of Analysis Periods for Well and Streamgage Data}

The 30-year period spanning water years 1990 to 2019 was used as the base period for calculating the mean waterlevel altitude for wells and mean base flow for streams (U.S. Geological Survey, 2020). To determine if this period of analysis was representative of long-term averages, mean values from water years 1990 through 2019 were compared with mean period-of-record values for the subset of wells (22 out of 65 ) and streamgages ( 8 out of 14 ) with periods of record that were complete during the base period and extended at least 1 year longer (tables 1.1 and 1.2). Well water-level records with an average of 10 or more measurements per year during each year of the base period were considered complete. Streamgage records with no missing daily values during the base period were considered complete.
For wells with complete records, mean depth to water for water years 1990 to 2019 ranged from 0.2 meter (m) shallower to $0.3 \mathrm{~m}$ deeper, with a median of $0.009 \mathrm{~m}$ shallower, than water levels for the periods of record (31 to 80 years, with a median of 52.5 years). For streamgages with complete records, mean base flow for water years 1990 to 2019 ranged from 3.2 percent lower to 5.2 percent higher, with a median of 0.2 percent lower, than mean base flow for the periods of record ( 37 to 91 years, with a median of 55 years). For this analysis, base flow was calculated by using the mean of base flow as calculated by the PART and BFI algorithms, as implemented in the DVstats package in R (Lorenz, 2017; R Core Team, 2019), and the mean of the two algorithms was used. Because the values for water years 1990 to 2019 differed from the

Table 1.1. U.S. Geological Survey groundwater-monitoring on the north shore of Long Island Sound (coastal Connecticut and adjacent areas of New York and Rhode Island) with long periods of record used for determining if water years 1990 to 2019 were representative of long-term average conditions.

[Map no. refers to number on figure 10. Water levels are reported in meters below land surface. Difference is between the mean water level for 1990 to 2019 and the mean water level for the period of record and is reported in meters. Water-level data for all wells are available in the National Water Information System (U.S. Geological Survey, 2020). CT, Connecticut; RI, Rhode Island]

\begin{tabular}{|c|c|c|c|c|c|c|}
\hline \multirow{2}{*}{$\begin{array}{c}\text { Map } \\
\text { no. }\end{array}$} & \multirow[b]{2}{*}{ Site number } & \multirow[b]{2}{*}{ Station name } & \multirow{2}{*}{$\begin{array}{l}\text { Period of record } \\
\text { (water years) }\end{array}$} & \multicolumn{2}{|c|}{ Mean water level } & \multirow[b]{2}{*}{ Difference } \\
\hline & & & & $\begin{array}{l}\text { Period of } \\
\text { record }\end{array}$ & $\begin{array}{l}1990 \text { to } 2019 \\
\text { (water years) }\end{array}$ & \\
\hline 11 & 411256073153101 & CT-FF 23 & 1966 to 2019 & 2.39 & 2.38 & -0.01 \\
\hline 15 & 412013072030601 & CT-GT 19 & 1958 to 2019 & 4.62 & 4.61 & -0.01 \\
\hline 16 & 412154071462901 & RI-WEW 522 WESTERLY, RI & 1966 to 2019 & 3.77 & 3.79 & 0.02 \\
\hline 17 & 412214071394001 & RI-CHW 18 & 1947 to 2019 & 5.4 & 5.36 & -0.04 \\
\hline 18 & 412307072515201 & CT-NHV 201 & 1975 to 2019 & 4.86 & 4.86 & 0 \\
\hline 23 & 412429073165101 & CT-NT 15 NEWTOWN, CT & 1967 to 2019 & 1.71 & 1.7 & 0 \\
\hline 30 & 412824072173301 & CT-SM 7 & 1979 to 2019 & 3.14 & 3.1 & -0.04 \\
\hline 31 & 412844071422802 & RI-RIW 600 RICHMOND, RI & 1977 to 2019 & 10.32 & 10.31 & -0.01 \\
\hline 33 & 412918071321001 & $\begin{array}{l}\text { RI-SNW } 6 \text { SOUTH } \\
\text { KINGSTOWN, RI }\end{array}$ & 1948 to 2019 & 3.69 & 3.61 & -0.08 \\
\hline 35 & 412932071374302 & RI-RIW 417 RICHMOND, RI & 1976 to 2019 & 2.08 & 2.08 & 0 \\
\hline 40 & 413007073250501 & CT-BD 8 & 1967 to 2019 & 9.25 & 9.24 & -0.02 \\
\hline 42 & 413134073021701 & CT-WB 93 & 1944 to 2019 & 8.29 & 8.29 & 0 \\
\hline 44 & 413148071281601 & RI-NKW 255 & 1954 to 2019 & 2.59 & 2.55 & -0.04 \\
\hline 45 & 413202073122401 & CT-WY 1 & 1914 to 2019 & 7.57 & 7.35 & -0.22 \\
\hline 46 & 413245072584201 & CT-WB 198 & 1944 to 2019 & 4.51 & 4.77 & 0.26 \\
\hline 47 & 413252071323601 & RI-EXW 554 EXETER, RI & 1989 to 2019 & 2.99 & 2.99 & 0 \\
\hline 49 & 413358071433801 & RI-EXW 475 EXETER, RI & 1981 to 2019 & 4.4 & 4.41 & 0 \\
\hline 52 & 413457072252201 & CT-CO 335 & 1986 to 2019 & 2.19 & 2.18 & -0.01 \\
\hline 55 & 413535072253701 & CT-MB 32 MARLBOROUGH, CT & 1986 to 2019 & 1.58 & 1.59 & 0.01 \\
\hline 57 & 413645071332901 & RI-WGW 206 & 1955 to 2019 & 1.37 & 1.38 & 0.01 \\
\hline 58 & 413907071465001 & RI-WGW 181 & 1966 to 2019 & 4.76 & 4.71 & -0.05 \\
\hline 59 & 414054071552001 & CT-PL 1 & 1943 to 2019 & 9.15 & 9.04 & -0.11 \\
\hline
\end{tabular}


Table 1.2. U.S. Geological Survey streamgages on the north shore of Long Island Sound (coastal Connecticut and adjacent areas of New York and Rhode Island) with long periods of record used for determining if water years 1990 to 2019 were representative of long-term average conditions.

[Base flow was calculated by using the PART and BFI algorithms as implemented in the DVstats package in R (Lorenz, 2017; R Core Team, 2019). Base flow is given in cubic meters per day. Difference is for 1990 to 2019 relative to the period of record and is reported in percent difference. Streamflow data for all gages are available in the National Water Information System (U.S. Geological Survey, 2020). CT, Connecticut; RI, Rhode Island]

\begin{tabular}{|c|c|c|c|c|c|}
\hline \multirow[b]{2}{*}{$\begin{array}{c}\text { Site } \\
\text { number }\end{array}$} & \multirow[b]{2}{*}{ Station name } & \multirow[b]{2}{*}{ Period of record } & \multicolumn{2}{|c|}{ Base flow } & \multirow{2}{*}{$\begin{array}{c}\text { Percent } \\
\text { difference }\end{array}$} \\
\hline & & & $\begin{array}{l}\text { Period of } \\
\text { record }\end{array}$ & $\begin{array}{l}1990 \text { to } 2019 \\
\text { (water years) }\end{array}$ & \\
\hline 01117468 & BEAVER RIVER NEAR USQUEPAUG, RI & $\begin{array}{r}1974-12-04 \text { to } \\
2019-09-30\end{array}$ & 41,038 & 39,723 & -3.2 \\
\hline 01117800 & WOOD RIVER NEAR ARCADIA, RI & $\begin{array}{r}1982-10-01 \text { to } \\
2019-09-30\end{array}$ & 143,613 & 142,557 & -0.7 \\
\hline 01118300 & $\begin{array}{l}\text { PENDLETON HILL BROOK NEAR CLARKS } \\
\text { FALLS, CT }\end{array}$ & $\begin{array}{r}1958-10-01 \text { to } \\
2019-09-30\end{array}$ & 13,339 & 13,672 & 2.5 \\
\hline 01123000 & LITTLE RIVER NEAR HANOVER, CT & $\begin{array}{r}1951-10-01 \text { to } \\
2019-09-30\end{array}$ & 85,724 & 85,416 & -0.4 \\
\hline 01193500 & SALMON RIVER NEAR EAST HAMPTON, CT & $\begin{array}{r}1928-10-01 \text { to } \\
2019-09-30\end{array}$ & 271,493 & 285,495 & 5.2 \\
\hline 01195100 & INDIAN RIVER NEAR CLINTON, CT & $\begin{array}{r}1981-11-04 \text { to } \\
2019-09-30\end{array}$ & 13,307 & 13,189 & -0.9 \\
\hline 01208950 & SASCO BROOK NEAR SOUTHPORT, CT & $\begin{array}{r}1964-10-01 \text { to } \\
2019-09-30\end{array}$ & 18,214 & 18,617 & 2.2 \\
\hline 01208990 & SAUGATUCK RIVER NEAR REDDING, CT & $\begin{array}{r}1964-10-01 \text { to } \\
2019-09-30\end{array}$ & 61,408 & 61,368 & -0.1 \\
\hline
\end{tabular}

period-of-record values by less than $0.5 \mathrm{~m}$ for wells and less than 5 percent for most streamgages, it was determined that the mean values calculated from data for water years 1990 to 2019 were representative of long-term average conditions.

The remaining wells (43 of 65) and streamgages (6 of 14) did not have complete records during the base period of water years 1990 through 2019, or their records did not extend beyond the base period. To determine if the shorter periods were representative of the long-term average conditions, mean values for these shorter periods were compared with the values for the full base period by using the 22 wells and 8 streamgages with complete records.

For the 22 wells with complete site records, mean water levels during the short periods (58 to 360 months of measurements, with a median of 311 months of measurements) ranged from $0.3 \mathrm{~m}$ shallower to $0.1 \mathrm{~m}$ deeper, with a median difference of $0.0 \mathrm{~m}$, than mean water levels during the base period of water years 1990 through 2019. Because the mean water levels during all the short periods were substantially similar to the mean water levels during the complete base period, the shorter periods were determined to be sufficiently representative of long-term average conditions and were included as observations.

For the eight streamgages with complete records, mean base flow during the six shorter periods ranged from 7 percent lower to 5 percent higher, with a median of 0.0 percent higher, than base flow during the base period of water years
1990 to 2019. At all eight gages, base flow was more than 3 percent lower during water years 1999 to 2016, and at three of the complete record sites (01195100, INDIAN RIVER NEAR CLINTON, CT; 01123000, LITTLE RIVER NEAR HANOVER, CT; and 01117800, WOOD RIVER NEAR ARCADIA, RI), base flow was more than 5 percent lower. This suggests that base-flow observations for the streamgage with data only during this period (01117370, QUEEN R AT LIBERTY RD AT LIBERTY RI) may be lower than longterm average conditions. Biases in the estimates of base flow could lead to biases in the estimates of groundwater recharge, hydraulic conductivity, and subsurface travel time. In this case, because the bias in the base-flow estimates (1) was relatively small (less than 10 percent) and (2) affected only one of the streamgages used in the calibration, it was determined that the implications of the bias on the regional model were likely minimal, and the gage was used in the calibration.

\section{References Cited}

Lorenz, D.L., 2017, DVstats-Functions to manipulate dailyvalues data (ver. 0.3.4): U.S. Geological Survey R software package, accessed June 24, 2021, at https://rdrr.io/github/ USGS-R/DVstats/. 
R Core Team, 2019, R-A language and environment for statistical computing (ver. 3.6.0): The R Foundation software release, accessed July 1, 2019, at http://www.R-project.org.
U.S. Geological Survey, 2020, USGS water data for the Nation: U.S. Geological Survey National Water Information System database, accessed February 7, 2020, at https://doi.org/10.5066/F7P55KJN. 


\section{Appendix 2. Estimation of Private-Well Withdrawals and Septic Return Flows}

Private-well withdrawals were estimated on the basis of service-area maps (Connecticut Office of Policy and Management, 2019), 2010 population density (Falcone, 2016), and per resident usage rates (Dieter and others, 2018). In some areas the service-area maps denoted areas served by public water, and in others they denoted the spatial footprint of the service lines. To better represent the areas served, two adjustments were made. For Connecticut, spatial data layers of public water service areas were intersected with parcel data, where available, to identify parcels that were served by public water. Second, for both Connecticut and adjoining areas of New York and Rhode Island, a 91-meter buffer was applied to the service-area data layer. The resulting maps of public water-supply areas maps from all three States were merged. The same process was used to generate a map of sewer service areas.

Calculations of well withdrawals and septic return flows were done at the model cell resolution. The availability of public water within each model cell was based on whether the center of the cell overlapped the map of public water-supply service areas. It was assumed that no private wells existed within the areas served by public water supply. The availability of public sewer was determined similarly for each model cell, and it was similarly assumed that septic systems did not exist within the area served by public sewer.

The population within each model cell was estimated on the basis of the average population density from a 2010 population density raster (Falcone, 2016) and the model-cell area. Using the raw population density raster, this approach accurately simulates the population-dependent well withdrawals and septic return flows at the scale of tens of kilometers. It does not, however, represent heterogeneity in withdrawals and return flows at scales of less than 1 kilometer because the population density raster reflects a smoother population distribution, with less clustering, than actually occurs. One artifact of the population smoothing is that the estimation algorithm resulted in noninteger populations within each model cell, some with noninteger populations well below 1. Enforcing integer values of population resulted in incorrect population totals, particularly in sparsely populated areas, because of rounding. For example, in an area with population density of 2 people per square kilometer, the number of people per model cell is approximately 0.5 . This could be rounded down to 0 people per cell, or up to 1 person per cell, with neither rounding method producing the correct population. Using the smooth, noninteger population distribution, however, resulted in many model cells with simulated private wells serving less than 1 person. To maintain the consistent total population and minimize the number of simulated wells serving an unrealistically small number of people, the estimated populations for cells with an estimated population less than 3 people but greater than 0 people were redistributed to nearby cells so that no cell had an estimated population between 0 and 3 . Three people was set as the threshold based on the average household size of 2.54 for Connecticut in 2014-18 (U.S. Census Bureau, 2019). The new location of redistributed population was selected by (1) proximity to the original cell, (2) presence of roads within the cell, and (3) estimated population within the new location.

Private well withdrawal rates for each model cell without available public water were calculated as the product of the population within the cell and a per resident usage rate of 0.18 cubic meters per day (Dieter and others, 2018). Septic return flow rates for each model cell without available public sewer were calculated as the product of the population within the cell, a per resident usage rate of 0.18 cubic meter per day (47 gallons per day) (Dieter and others, 2018) and a consumptive-use fraction of 0.15 (Shaffer and Runkle, 2007). No assumption was made regarding the co-occurrence of public water and public sewer. Calculations of private-well withdrawals and septic return flows were calculated separately, and all four possible combinations exist within the study area: public water and public sewer, public water and septic system, private well and public sewer, and private well and septic system.

\section{References Cited}

Connecticut Office of Policy and Management, 2019, Public water service areas 2017 (PublicWaterService2017.shp): State of Connecticut dataset, accessed December 2, 2019, at ftp://ftp.state.ct.us/pub/opm/TCDPP/C\&D\%20Plan $\% 20$ GIS/GIS/OPM_DRAFT_C\&D_Data_Web/WaterService/.

Dieter, C.A., Linsey, K.S., Caldwell, R.R., Harris, M.A., Ivahnenko, T.I., Lovelace, J.K., Maupin, M.A., and Barber, N.L., 2018, Estimated use of water in the United States county-level data for 2015: U.S. Geological Survey data release, accessed June 24, 2021, at https://doi.org/10.5066/ F7TB15V5.

Falcone, J.A., 2016, U.S. block-level population density rasters for 1990, 2000, and 2010: U.S. Geological Survey data release, accessed June 24, 2021, at https://doi.org/10.5066/ F74J0C6M.

Shaffer, K.H., and Runkle, D.L., 2007, Consumptive water-use coefficients for the Great Lakes Basin and climatically similar areas: U.S. Geological Survey Scientific Investigations Report 2007-5197, 191 p., accessed June 24, 2021, at https://doi.org/10.3133/sir20075197.

U.S. Census Bureau, 2019, 2014-2018 American Community Survey 5-year estimates: U.S. Census Bureau dataset, accessed July 22, 2020, at https://www.census.gov/ quickfacts/fact/table/CT/PST045219. 


\section{Appendix 3. Estimation of Stream Width}

The stream width was estimated by using a geomorphic scaling relationship, which was developed from the sum of upstream river lengths (the arbolate sum), as recorded in the National Hydrography Dataset Plus (NHDPlus; U.S. Environmental Protection Agency and U.S. Geological Survey, 2012), and the stream width, as recorded in the National Water Information System (U.S. Geological Survey, 2020), for 204 sites in New England (hydrologic region 01) (Seaber and others, 1987). This analysis follows the example used by Feinstein and others (2010, app. 2, p. 264).

First, all sites with a recorded stream width (parameter code 00004) were selected, and the mean stream width was calculated. Then the sites were spatially joined using ArcMap and Python to the closest NHDPlus segment, and the arbolate sum (total upstream length of mapped streams) was extracted. The stream width was plotted against the arbolate sum on a semilog plot, and outliers were examined. Three sites were removed that were joined to the incorrect stream segment. The final site list included 204 sites and is available in Barclay and Mullaney (2021). The stream orders ranged from 1 to 6 , with a median of 3 . The arbolate sums ranged from 1.0 to $19,023.8$ kilometers, with a median of 62.3 kilometers. The stream widths ranged from 0.61 to 291.7 meters, with a median of 8.2 meters.

The natural logarithm of the stream width and arbolate sum data were fit to a linear-regression equation of the following form:

$$
\ln (w)=a+b * \ln (s),
$$

where

$$
\begin{aligned}
& w \quad \text { is the width of the stream, in meters; } \\
& s \quad \text { is the arbolate sum of upstream river lengths, } \\
& \text { in kilometers; } \\
& a \quad \text { is a regression coefficient; and } \\
& b \quad \text { is a regression coefficient. }
\end{aligned}
$$

The linear-equation coefficients were calculated to be $a=-0.20$ and $b=0.5594$. The coefficient of determination $\left(R^{2}\right)$ value for the regression equation was 0.83 (fig. 3.1). To estimate the stream width, the coefficients were inserted into equation 3.1, and the equation was rearranged to yield

$$
w=\mathrm{e}^{-0.20} S^{0.5594},
$$

where

$w \quad$ is the width of the stream, in meters;

e is Euler's number, 2.71828; and

$s \quad$ is the arbolate sum of upstream river lengths, in kilometers.

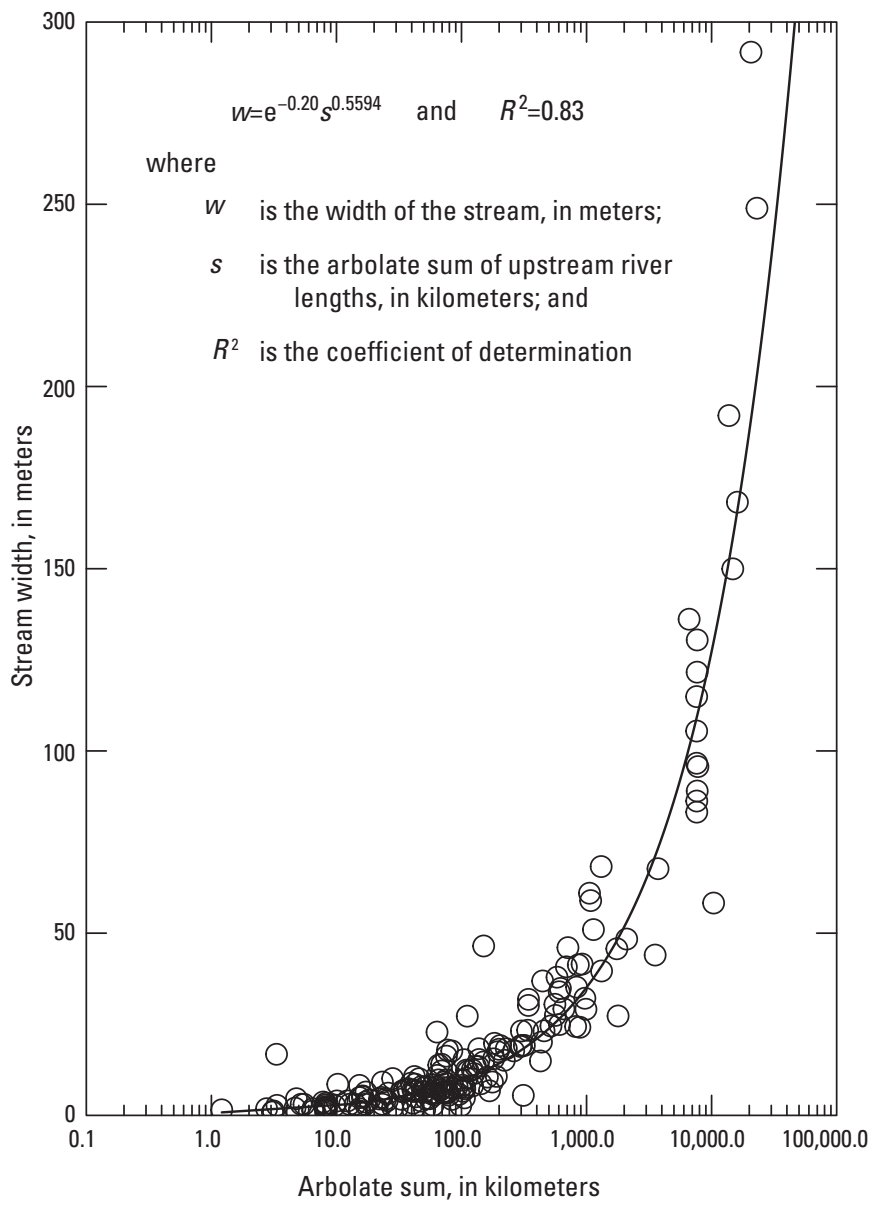

\section{EXPLANATION}

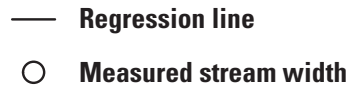

Figure 3.1. Scatterplot of measured stream width and the cumulative length of upstream flowlines (arbolate sum) for sites in New England. 


\section{References Cited}

Barclay, J.R., and Mullaney, J.R., 2021, Summary data on groundwater budgets and travel times for watersheds on the north shore of Long Island Sound: U.S. Geological Survey data release, https://doi.org/10.5066/P91TQ895.

Feinstein, D.T., Hunt, R.J., and Reeves, H.W., 2010, Regional groundwater-flow model of the Lake Michigan Basin in support of Great Lakes Basin water availability and use studies: U.S. Geological Survey Scientific Investigations Report 2010-5109, 379 p., accessed June 24, 2021, at https://doi.org/10.3133/sir20105109.
Seaber, P.R., Kapinos, F.P., and Knapp, G.L., 1987, Hydrologic unit maps: U.S. Geological Survey Water Supply Paper 2294, 63 p., 1 pl., accessed June 25, 2021, at http://pubs.er.usgs.gov/publication/wsp2294.

U.S. Environmental Protection Agency and U.S. Geological Survey, 2012, National Hydrography Dataset PlusNHDPlus (ver. 2.10): U.S. Environmental Protection Agency and U.S. Geological Survey dataset, accessed June 24, 2021, at https://www.epa.gov/waterdata/nhdplusnational-hydrography-dataset-plus.

U.S. Geological Survey, 2020, USGS water data for the Nation: U.S. Geological Survey National Water Information System database, accessed February 7, 2020, at https://doi.org/10.5066/F7P55KJN. 
For more information about this report, contact: Director, New England Water Science Center

U.S. Geological Survey

10 Bearfoot Road

Northborough, MA 01532

dc_nweng@usgs.gov

or visit our website at

https://www.usgs.gov/centers/new-england-water

Publishing support provided by the Pembroke, Lafayette, and Moffett Field Publishing Service Centers 


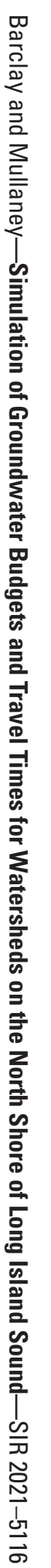

